University of Louisville

ThinkIR: The University of Louisville's Institutional Repository

Electronic Theses and Dissertations

$12-2020$

\title{
Computational behavioral analytics: estimating psychological traits in foreign languages.
}

Kristopher Wayne Reese

University of Louisville

Follow this and additional works at: https://ir.library.louisville.edu/etd

Part of the Data Science Commons

\section{Recommended Citation}

Reese, Kristopher Wayne, "Computational behavioral analytics: estimating psychological traits in foreign languages." (2020). Electronic Theses and Dissertations. Paper 3568.

https://doi.org/10.18297/etd/3568

This Doctoral Dissertation is brought to you for free and open access by ThinkIR: The University of Louisville's Institutional Repository. It has been accepted for inclusion in Electronic Theses and Dissertations by an authorized administrator of ThinkIR: The University of Louisville's Institutional Repository. This title appears here courtesy of the author, who has retained all other copyrights. For more information, please contact thinkir@louisville.edu. 


\title{
COMPUTATIONAL BEHAVIORAL ANALYTICS: ESTIMATING PSYCHOLOGICAL TRAITS IN FOREIGN LANGUAGES
}

\author{
By \\ Kristopher Wayne Reese \\ M.S., University of Louisville, 2011 \\ B.S., Hood College, 2009 \\ B.A., Hood College, 2009 \\ A Dissertation \\ Submitted to the Faculty of the \\ J.B. Speed School of Engineering of the University of \\ Louisville \\ in Partial Fulfillment of the Requirements \\ for the Degree of \\ Doctor of Philosophy \\ in Computer Science and Engineering \\ Computer Science and Engineering \\ University of Louisville \\ Louisville, Kentucky
}

December 2020 
Copyright 2020 by Kristopher Wayne Reese

All rights reserved 



\title{
COMPUTATIONAL BEHAVIORAL ANALYTICS: ESTIMATING
} PSYCHOLOGICAL TRAITS IN FOREIGN LANGUAGES

\author{
By \\ Kristopher Wayne Reese \\ M.S., University of Louisville, 2011 \\ B.S., Hood College, 2009 \\ B.A., Hood College, 2009 \\ Dissertation approved on
}

October 23, 2020

by the following dissertation Committee:

\begin{tabular}{c}
\hline $\begin{array}{c}\text { Dissertation Director } \\
\text { Adel Elmaghraby }\end{array}$ \\
\hline
\end{tabular}

Roman Yampolskiy, CSE

Dar-Jen Chang, CSE

Adrian Lauf, CSE

Michael Losavio, Criminal Justice 


\section{DEDICATION}

For my wife, Danielle, and my son, Ezra. Thank you for all of your support and love through all of the work that went into this dissertation. 


\section{ACKNOWLEDGMENTS}

I'd like to thank all the people who have helped me throughout the dissertation process including my advisor, Dr. Adel Elmaghraby, who stood with me through the long process. Thank to the various Government colleagues who provided assistance in algorithm development and for helping to teach me various statistical techniques used in analysis. I'd also like to acknowledge my colleagues at the University of Florida, especially Dr. Damon Woodard, who helped with aspects of the authorship attribution experiments explored in this dissertation.

This dissertation makes use of various Free and Open Source Python 3 libraries for Natural Language Processing, Machine Learning algorithm development, and data analysis.

The research made use of data provided by the Linguistic Data Consortium, the University of Cambridge, and North Carolina Agricultural \& Technical State University. 


\section{ABSTRACT \\ COMPUTATIONAL BEHAVIORAL ANALYTICS: ESTIMATING PSYCHOLOGICAL TRAITS IN FOREIGN LANGUAGES \\ Kristopher Wayne Reese}

December 12, 2020

The rise of technology proliferating into the workplace has increased the threat of loss of intellectual property, classified, and proprietary information for companies, governments, and academics. This can cause economic damage to the creators of new IP, companies, and whole economies. This technology proliferation has also assisted terror groups and lone wolf actors in pushing their message to a larger audience or finding similar tribal groups that share common, sometimes flawed, beliefs across various social media platforms. These types of challenges have created numerous studies in psycholinguistics, as well as commercial tools, that look to assist in identifying potential threats before they have an opportunity to conduct malicious acts. This has led to an area of study that this dissertation defines as "Computational Behavioral Analytics."

A common practice espoused in various Natural Language Processing studies (both commercial and academic) conducted on foreign language text is the use of Machine Translation (MT) systems before conducting NLP tasks. In this dissertation, we explore three psycholinguistic traits conducted on foreign language text. We explore the effects (and failures) of MT systems in these types of psycholinguistic tasks in order to help push the field of study into a direction that will greatly improve the efficacy of such systems. 
Given the results of the experimentation in this dissertation, it is highly recommended to avoid the use of translations whenever the greatest levels of accuracy are necessary, such as for National Security and Law Enforcement purposes. If translations must be used for any reason, scientist should conduct a full analysis of the impact of their chosen translation system on their estimates to determine which traits are more significantly affected. This will help ensure that analysts and scientists are better informed of the potential inaccuracies and change any resulting decisions from the data accordingly.

This dissertation introduces psycholinguistics and the benefits of using Machine Learning technologies in estimating various psychological traits, and provides a brief discussion on the potential privacy and legal issues that should be addressed in order to avoid the abuse of such systems in Chapter I. Chapter II outlines the datasets that are used during the experimentation and evaluation of the algorithms. Chapter III discusses each of the various implementations of the algorithms used in the three psycholinguistic tasks - Affect Analysis, Authorship Attribution, and Personality Estimation. Chapter IV] discusses the experiments that were run in order to understand the effects of MT on the psycholinguistic tasks, and to understand how these tasks can be accomplished in the face of MT limitations, including rationale on the selection of the MT system used in this study. The dissertation concludes with Chapter $\mathrm{V}$, providing a discussion and speculating on the findings and future experimentation that should be done. 


\section{TABLE OF CONTENTS}

Page

Dedication . . . . . . . . . . . . . . . . . iii

Acknowledgments . . . . . . . . . . . . . . . . . . . iv

Abstract . . . . . . . . . . . . . . . . . . . . . v v

List of Tables . . . . . . . . . . . . . . . . . . . . $\mathrm{x}$

List of Figures $\ldots \ldots \ldots \ldots$. . . . . . . . . . . . . . . . . . . . . . .

Introduction . . . . . . . . . . . . . . . . . . . . . . . . . . 1

Computational Behavioral Analytics . . . . . . . . . . . . 3

Machine Learning \& Psychology . . . . . . . . . . . . . . . . . . 4

Incipient and Enduring Threats . . . . . . . . . . . . . . . 6

Insider Threats . . . . . . . . . . . . . . . . . . . 6

Terrorism, Radicalization, and Extremism … . . . . . . 10

Deanonymization of Threats . . . . . . . . . . . . . 13

Privacy and Legal Issues . . . . . . . . . . . . . . . . . . 16

Datasets . . . . . . . . . . . . . . . . . . . . . 18

Affect Analysis $\ldots \ldots \ldots \ldots \ldots \ldots$

English Language Datasets . . . . . . . . . . . . . . . . . . 22

Foreign Language Dataset . . . . . . . . . . . . . . . . 25 
Personality Trait Estimation . . . . . . . . . . . . . . . . . . . . 31

Personality Dataset . . . . . . . . . . . . . . . . . 35

Identifying Non-English users . . . . . . . . . . . . . . . . . 39

Authorship Attribution . . . . . . . . . . . . . . . . . . 42

BOLT Datasets . . . . . . . . . . . . . . . . . . . . 43

Choosing users . . . . . . . . . . . . . . . . 45

Linguistic Tasks . . . . . . . . . . . . . . . . . . . . . . . . 50

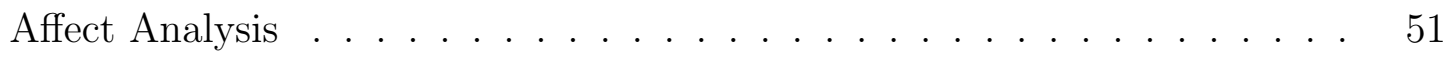

Algorithm Design . . . . . . . . . . . . . . . . . . . 53

English Performance . . . . . . . . . . . . . . . . . . 54

Arabic Performance . . . . . . . . . . . . . . . . . . . 58

Personality Trait Estimation . . . . . . . . . . . . . . . . . . . 61

Algorithm Design . . . . . . . . . . . . . . . . . . . . 64

Authorship Attribution . . . . . . . . . . . . . . . . . . . . 68

Algorithms. . . . . . . . . . . . . . . . . . . . . 69

English Performance . . . . . . . . . . . . . . . . . . . 72

Effects of Translation . . . . . . . . . . . . . . . . . . . . 77

Data Translations . . . . . . . . . . . . . . . . . . . . 78

Affect Dataset . . . . . . . . . . . . . . . . . . . . . . . . 79

Personality Dataset . . . . . . . . . . . . . . . . . . . . . 79

Authorship Dataset . . . . . . . . . . . . . . . . . . . 81

Affect Analysis $\ldots \ldots \ldots \ldots \ldots \ldots$. . . . . . . . . . . . . 81

Error Analysis . . . . . . . . . . . . . . . . . . . 83

Personality Trait Estimation . . . . . . . . . . . . . . . . 88

Error Analysis . . . . . . . . . . . . . . . . . . . . . . . 9 90

Impact on Feature Distributions . . . . . . . . . . . . . . . . 96 
Impact on Readability Measures . . . . . . . . . . . . . . . . . . . . . 97

Authorship Attribution . . . . . . . . . . . . . . . . . . 98

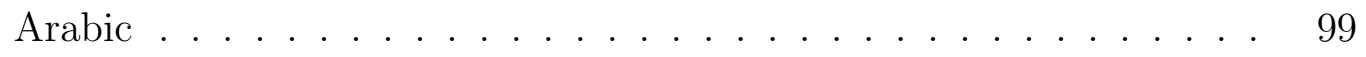

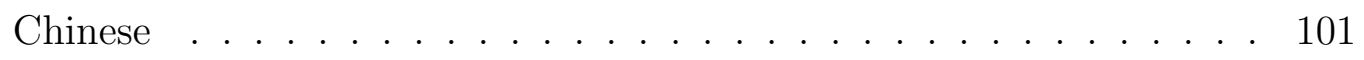

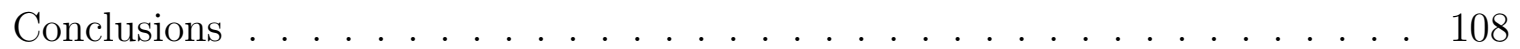

Discussions and Speculations _. . . . . . . . . . . . . . 109

Impact . . . . . . . . . . . . . . . . . . . . . . 111

Future Research Efforts . . . . . . . . . . . . . . . . . . . . . . . 112

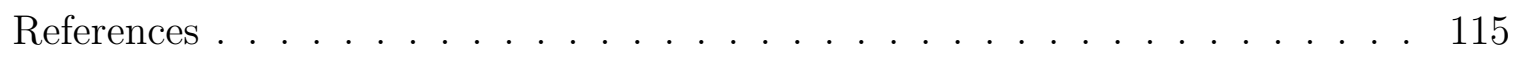

Appendix A: Feature Significance Table . . . . . . . . . . . . . . . . 133

Curriculum Vitae . . . . . . . . . . . . . . . . . . . . . . . . . . . . 182 


\section{LIST OF TABLES}

$1 \quad$ Median Annotator Agreement . . . . . . . . . . . . . . . . . . . . 27

2 Makeup of the Emotion dataset collected . . . . . . . . . . . . . 30

3 Dataset Statistics for FFM traits against all data. . . . . . . . . . . 36

$4 \quad$ Dataset Statistics for FFM traits for users with status updates. . . . . . 36

5 Dataset Statistics for FFM traits for Foreign Language Authors. . . . . . 42

$6 \quad$ User Counts by number of posts. . . . . . . . . . . . . . . . . . 44

$7 \quad$ Statistics of Translated BOLT Data. . . . . . . . . . . . . . . 46

$8 \quad$ Grid Search Optimization of English Features $\ldots \ldots \ldots \ldots$

$9 \quad$ Optimized Hyperparameters for each English emotion model . . . . . . . 57

$10 \quad$ Metrics for optimized English emotion models $\ldots \ldots \ldots$. . . . . . . . 57

11 Grid Search Optimization of Arabic Features . . . . . . . . . . . . 60

12 Optimized Hyperparameters for each Arabic emotion model . . . . . . . 60

13 Metrics for optimized Arabic emotion models $\ldots \ldots \ldots$

14 RMSE for tested algorithms . . . . . . . . . . . . . . . . . . 65

15 RMSE for number of topics tested . . . . . . . . . . . . . . 66

$16 \quad$ RMSE for with additional NLP Features $\ldots \ldots \ldots$. . . . . . . 67

17 Languages identified in personality data $\ldots \ldots \ldots \ldots 78$

18 Significance Testing of Emotion data $\ldots \ldots \ldots \ldots$

19 Correlation Analysis of Emotion data . . . . . . . . . . . . . . . 87

20 Significance Testing of Emotions' squared error $\ldots \ldots \ldots . . \ldots 88$

21 Medians of the Error Squared distributions . . . . . . . . . . . . . 88 
$22 \quad$ Significance Testing of multi-lingual personality data . . . . . . . . . . . 91

23 Significance Testing of no English personality data . . . . . . . . . . . . 94

24 Significance Testing on Translations per Language . . . . . . . . . . . . . 105

$25 \quad$ Significance Testing on Translations per Linguistic Family . . . . . . . . 106

26 Significance Testing on readability metrics of translations . . . . . . . . . 106

27 Basic Statistics for the Arabic EER Distributions . . . . . . . . . . . . . 106

28 Basic Statistics for the Chinese EER Distributions . . . . . . . . . . . . . 107 


\section{LIST OF FIGURES}

$1 \quad$ Plutchik's Wheel of Emotions and Dyads . . . . . . . . . . . . . . . . 22

2 Qualification Test Question Example. . . . . . . . . . . . . . . . 27

3 Histogram showing equivalency classes for intensity scores. . . . . . . . . 30

$4 \quad$ Posts per user for Personality subset. . . . . . . . . . . . . . . . . . 37

5 OCEAN Traits for all users in dataset. . . . . . . . . . . . . 38

$6 \quad$ OCEAN Traits for subset with status posts. . . . . . . . . . 38

$7 \quad$ Histogram showing the age of users in the dataset. $\ldots \ldots \ldots$

8 Histogram showing the age of Foreign Language users. . . . . . . . . . . 40

$9 \quad$ Posts per user for Foreign Language Personality subset. . . . . . . . . . . . 41

$10 \quad$ OCEAN Traits for Foreign Language data. . . . . . . . . . . . . . . . 41

$11 \quad$ Arabic Histogram for Post counts per user. . . . . . . . . . . . . . . . 48

12 Chinese Histogram for Post counts per user. . . . . . . . . . . . . . . . 49

13 Word Sensitivity Curve for Personality Trait Estimation . . . . . . . . 68

14 Authorship Attribution Algorithm Accuracy . . . . . . . . . . . . . 73

15 ROC Curve for Keselj Algorithm on Various Sentence Lengths . . . . . . 75

16 Genuine vs. Imposter Charts . . . . . . . . . . . . . . . . . 76

17 Error Distributions for Emotions. . . . . . . . . . . . . . . . . 83

18 Translation Error Distributions for Emotions. . . . . . . . . . . . . . . 84

19 Error Squared Distributions for Emotions. . . . . . . . . . . . . . . 85

$20 \quad$ Translation Error Squared Distributions for Emotions. $\ldots . . . . . . .86$

21 Character Count for Personality Data . . . . . . . . . . . . . . . . . 90 
$22 \quad$ Error distributions for English OCEAN Traits. . . . . . . . . . . . . . . . 92

23 Error Squared distributions for English OCEAN Traits. . . . . . . . . . . 92

24 Error distributions for Translation OCEAN Traits. . . . . . . . . . . . . 93

$25 \quad$ Error Squared distributions for Translation OCEAN Traits. . . . . . . . 93

26 Mean ROC curve for Arabic Dataset. . . . . . . . . . . . . . . . . . . . . 100

27 EER Histogram for Arabic Dataset. . . . . . . . . . . . . . . . . . . . . . 100

28 Mean ROC curve for the Chinese Dataset. . . . . . . . . . . . . . . . . . 102

$29 \quad$ EER Histogram for the Chinese Dataset. . . . . . . . . . . . . . . . . . . 102 


\section{CHAPTER I}

\section{INTRODUCTION}

"Computational Behavioral Analytics" (CBA) is a subset of computational social sciences focused on using Machine Learning and Artificial Intelligence to estimate psychological and cognitive features to understand and add context to the behaviors, activities, and motivations of the human behind observed data. Our daily communications have increasingly shifted to written text on technological tools - text messaging on cell phones, online messaging services, email services, or social media platforms. Understanding the people behind these tools is a modern challenge that businesses and government agencies are tasked with to identify potential indicators of threats to their intellectual property and their task to protect their people.

The increase of technology proliferating in the workplace has increased the threat of intellectual property loss, classified information, and proprietary information for companies, governments, and academics. This rise in technologies can cause economic damage to the creators of new Intellectual Properties, companies, and whole economies. This technology proliferation throughout our culture has also assisted terror groups, lone-wolf actors, and nation-states in pushing their messages to broader audiences, the spreading of misinformation, or finding similar tribal groups that share common, often intolerant, beliefs across various social media platforms. As law enforcement, intelligence agencies, and businesses seek out possible threats, the amount

of data available for psychologists to review grows beyond the number of psychologists 
available to each organization. Psychologists provide methods and techniques for understanding the behaviors of malicious actors. These psychologists provide methods for understanding the behaviors of malicious actors. Research and Development of new tools have provided commercial applications that estimate psychological traits and monitor for potential threats, such as IBM's QRadar [1], for detecting insider threats; IBM's Personality Insights [2], for estimating Personality Traits; and Crystal Knows [3], for estimating group dynamics using DISC profiles.

As our economies and national security threats become globalized, the use of foreign language threats to businesses and countries rises, many of the tools are reliant on Machine Translations to help in understanding the messages conveyed by people. Organizations have done little or no research on the impact of Machine Translations on these psychological tools when attempting to understand human behavior. This dissertation looks at several cutting edge machine learning models for estimating emotional intensity, personality traits, and authorship styles and determine how these models are affected by machine translations.

In this chapter of the thesis, we introduce the rich history between Machine Learning and Cognitive and Psychological Sciences, as well as existing studies in psycholinguistics attempting to understand psychological traits. The chapter also delves into the various reasons such a field exists, focusing on the needs of businesses and government agencies. It mainly focuses on the impact such a field could have on Insider Threats to organizations, Counterproductive work behaviors, and identification of potential radicalization methods. A discussion of some of the potential privacy issues that could arise in "Computational Behavioral Analytics" concludes the chapter. Chapter III explores each of the machine learning models in greater detail, giving an introduction to each of the psychological theories underpinning the work. It also discusses each of the machine learning models employed in greater detail. Chapter [I] explores the datasets used in training in greater detail and discuss methods for 
expanding the datasets in the future, offering methods for collection used in future studies. Chapter IV discusses the experimental design and results of the experiments to understand the impact of Machine Translation on the algorithms to determine the viability of using translations for estimating psychological traits. The dissertation concludes by discussing the results of the experiments and future research.

\section{Computational Behavioral Analytics}

The field is rife with possibilities to open up an understanding of people through the use of psycholinguistics, cognitive studies that mathematically model cognitive functions, or the use of computer vision for estimating psychological or cognitive functions hidden in collected data. While there has been some work in bringing Machine Learning into psychology, the field is still relatively nascent. These studies often suffer from a relatively low number of properly ground-truthed datasets. At the same time, these studies challenge researchers to build sufficiently large humanderived datasets to make Machine Learning viable in the field. Many datasets rely on psychologists' expertise to train models, but this method is untenable due to the limited availability of psychologists and the amount of time needed to train large datasets sufficiently. Ideally, the field would rely on subjects taking various validated psychological or cognitive assessments, but this requires significant effort to collect data. However, building datasets using these validated instruments disperses the time needed by experts to the subjects themselves, making the collection of more massive datasets viable for use in Machine Learning models.

Using either form of dataset introduced some form of bias into a machine learning system, making the understanding of an individual more challenging. Using datasets created by experts, the Machine Learning models determine the traits based on how the experts analyzed the persona that a subject in portraying to the experts. The use of psychological instruments can suffer from unintentional or intentional gaming 
of the instruments in an attempt to hide their real personality. However, psychologists have long dealt with the problem by adding inversions of questions to identify those subjects who may be attempting to game the instruments. Using psychological instruments for building datasets allows Machine Learning models to estimate traits based on how the subject answers the questions.

Despite the challenges and the current lack of sizeable datasets, studies into the field of "Computational Behavioral Analytics" continue to occur, and continued progress adds to the understanding of various aspects of human psychology. However, significant effort needs to occur to make the field as cross-disciplinary as it ought to be. Many studies often occur without consulting researchers outside of their fields of study. Various studies exist in computer science that delves into psychological traits, but these often lack any consultation of psychologists in helping to interpret the data. In the same vein, the use of Machine Learning in Psychology has taken off, often limited to older models that might be inferior to the tasks at hand. Consultation of Computer Scientist and Mathematicians might increase model efficiency and accuracy.

\section{Machine Learning \& Psychology}

Machine learning and cognitive science have a rich history together. The fields grew from a postwar movement away from the behaviorist paradigm that dominated cognition research during the first half of the twentieth-century [4]. Some of the preeminent names in Artificial Intelligence, such as Allen Newell and Herbert Simon, were cognitive scientists who developed new algorithms in AI in an attempt to model human processes. Up until the 1970s, Artificial Intelligence and Cognitive sciences often shared conferences where the two paths began to diverge. Since this schism, cognitive scientists and artificial intelligence researchers have shared methodologies, but often with very different goals. Jerome Feldman [4] identifies the differences between 
the two fields as AI's search for an understanding of intelligent behaviors, whether applied to animals or artificial systems, whereas cognitive science has a more narrow goal of understanding human intelligence.

Because of the differences, Cognitive Sciences tend to be much more interdisciplinary, incorporating linguistics, psychology, and artificial intelligence using computation as the universal language that could assist in the understanding of the human mind [5]. It is only recently that artificial intelligence and machine learning have reached a point where it can begin to contribute as a common language for many different fields, including in the study of human behaviors. The recent advancement of Artificial Intelligence and Machine Learning is needed because of the increase in the use of technologies for communications.

According to Raconteur [6], humans will produce 463 Exabytes of data every day by 2025. Even in 2019, society is producing massive amounts of data per day: nearly 500 million tweets, sending 294 billion emails, 65 billion WhatsApp messages, and generating over 4 Petabytes of Facebook data, consisting of 350 million photos, 100 million hours of video content, and other social media postings on the platform [6]. Sifting through this data would require a massive number of psychologists to identify potential threats. In 2012, the American Psychological Association estimated that there are 106,500 licensed clinical psychologists in the United States [7]. Without increasing the number of psychologists, by 2025, each psychologist would have to go through 4.3 Petabytes of data per day in search of potential threats. This amount of data also assumes that all licensed clinical psychologists have dedicated their time to identify threats, which would take them away from their medical duties.

Despite this need for more clinical psychologists, there is a shortage of psychologists around the world, even within the medical facilities of Malaysia and Australia [8]. Artificial intelligence and machine learning can help psychologists and play a more significant role in the behaviorist paradigm for helping psychologists understand 
the motivations, activities, and behaviors of people, especially in an ever-increasing virtual world.

\section{Incipient and Enduring Threats}

The expansion of technologies as a communications medium has raised incipient risks and threats to consumers. Cyber-Psychologists have identified exposure to sexually explicit material and child pornography [9, victimization through harassment and bullying [10], and internet addiction [11, 12, 13, 14] as some of the risks that modern consumers face on these technologies. The concept of internet-induced anonymity has also given rise to issues of deception in online environments, often assisted by the inability to verify information [15]. While many online platforms have taken steps to minimize deception that leads to significant financial or social losses, deception of identity is a growing social problem, even in online dating platforms [16]. While the magnitude of online dating deception is usually minor [17], online deception can sometimes lead to more extreme issues such as homicide, financial losses, or online predation.

\section{Insider Threats}

While technological developments create new and emerging threats, it has also reshaped ongoing and enduring threats posed against society. Both Psychology and Computer Scientists have identified Insider Threats as an issue for businesses. The term "Insider Threats" is a term often used by Computer Emergency Response Teams (CERTs). Carnegie Mellon's Insider Threat Team [18] has defined an insider threat as, "a current or former employee, contractor, or other business partners who has or had authorized access to an organization's network, system, or data and intentionally misused that access to negatively affect the confidentiality, integrity, or availability of the organization's information or information systems." [19] Psychologists have taken 
a much broader definition and include the CERT definition of Insider Threats under the concept of "Counterproductive Work Behaviors." According to [20], "Counterproductive Work Behaviors (CWBs) include actions that harm organizations such as absenteeism, substance abuse on the job, and theft (i.e., organization CWBs), as well as harassment and aggressive acts directed towards coworkers (i.e., interpersonal CWBs)."

Insider threats have almost certainly always existed in companies; it is with new technologies that insider threats have been more able to steal Intellectual Property, trade secrets, or large quantities of classified information from government agencies. These acts damage businesses and whole economies and potentially threaten the financial and physical safety of citizens. Another enduring threat is terrorism. Today's Internet and communication tools have created a new landscape for groups to radicalize vulnerable people and incite violence towards those who share different beliefs.

The Commission on the Theft of American Intellectual Property [21] conservatively estimates the damages to the U.S. economy, caused by intellectual property theft, to be between $\$ 225$ billion and $\$ 600$ billion. These numbers are conservative because estimating the damages caused is challenging due to the ease of copying and distributing intellectual property because of technological advancements. In 2012, the U.S. Congress estimated the revenue loss to a company to be, on average, about $\$ 101.9$ million a year[22].

According to findings of a 2018 Threat Report from Cybersecurity Insiders [23], most businesses feel vulnerable to insider threats, while $53 \%$ of surveyed businesses have confirmed insider attacks against their organization. In 2020, $68 \%$ of businesses have claimed that insider attacks are occurring more frequently, and the transition to cloud infrastructures has made identifying insider threats more difficult.

The Software Engineering Institute at Carnegie Mellon has created an Insider Threats team dedicated to researching Insider Threats at government agencies and 
businesses. A 2012 study from the group [24], found several motivations that drive people to commit insider attacks, especially in the financial sector: revenge against the organization for perceived injustice done to the attacker [25]; those who believe that they own the Intellectual Property because of the work that they have put into it [26]; those who can recruit insiders to steal information that they may not have authorized access to, often for some larger purpose[26]; and financial gain caused by financial stress of the employee [25]. Despite the identification of these potential motivations, the group has not considered those individuals who may have similar motivations but never act on it during this study.

Numerous psychological experiments have explored the relationship between personality traits and counterproductive workplace behaviors (CWBs) [27, 28, 29, 30, 31, 20, 32]. In psychological studies, the organizational perspective (CWB-O) [33], defining CWB as acts that go against an organization's interests, including those activities that may involve verbal and physical attacks against coworkers. Other literature takes a more employee-centric perspective (CWB-I), often looking at CWBs as behaviors that harm or intend to harm others or organizations [34].

Studies often explore the relations between CWBs and the Five-Factor Model (FFM), though additional models, such as the HEXACO model, are not uncommon in literature. The FFM classifies personality traits into five categories: Extraversion, Agreeableness, Conscientiousness, Neuroticism, and Openness (the Five-Factor Model is discussed further in Chapter II. The primary difference between the FFM and the HEXACO model is the inclusion of a humility trait within the HEXACO model. Broader studies are still needed, but several studies have identified low Agreeableness and high neuroticism as correlated with Organizational CWBs (such as stealing and absenteeism), and low Agreeableness with Interpersonal CWBs (such as harassment and aggression) [30, 35]. Berry et al. [36] also found a strong correlation between low Agreeableness and low Conscientiousness related to overall CWBs. The correlation 
of personality traits to CWBs appears to remain constant over several years. Le et al. [20] found that assessments of personality traits in adolescence appear to be predictive of CWBs in adulthood still. Over time, this stability of personality traits makes the psychological paradigm a potentially useful tool to help add additional context in understanding potential insider threats and other CWBs.

Psychologists studied the relationship between FFM traits and various forms of CWB. Bolton, Becker, and Barber's study [37] confirmed previous findings with the relationship of Agreeableness with CWB-I and Conscientiousness with CWB-O. However, this same study found that Conscientiousness is only predictive of behaviors of sabotage and theft. They also found that extraversion is predictive of theft, and Openness can help to predict production deviance. Some studies have shown that it is the interaction of traits that allow for more accurate predictions of CWBs [38].

Computer Scientists and Electrical Engineers have only recently begun to explore the predictive ability of personality traits to determine potential insider threats to an organization. However, due to a lack of data, situations are often simulated. A 2019 study from Singapore used games to simulate a world allowing them to put a subject in an environment that allows them to commit insider attacks while collecting personality traits, facial expressions, and linguistic features to identify Intellectual Property theft behaviors [39]. This type of simulation can cause issues when the reward structure does not closely match the real-world; this can cause inconsistencies in identifying potential insider threats to an organization.

Recent studies have attempted to bridge the divide between Computer Scientists and Psychologists. Maasberg et al. [40] looked at correlations of Dark Triad traits and Insider threats. Other studies have looked at Information-Theoretic approaches for measuring the "Trustworthiness" of employees [41] or the creation of Ontological methods for determining insider threats [42]. In 2018, Yang et al. [43] began to explore the use of a more comprehensive system utilizing various psychological models. 
All of these psychological and computer science studies into the problem contribute to our understanding of insider threats, but the systems developed are still heavily reliant on linguistic processing. Many of these tools have not considered how large multi-national corporations might attempt to identify insider threats when non-English languages are present.

\section{Terrorism, Radicalization, and Extremism}

Terrorism is another enduring threat that faces the national security and safety of many people around the world. The U.S. National Intelligence Council says that terrorism is likely to increase over the next $5-20$ years due to alienation, ethnic bonds in their networks, the loss of connection with their community of origin among immigrants, and ethnic and religious tensions [44]. Technological advancements allow terrorist groups to mask their identities and activities, recruit new members, finance their operations, and disseminate their messages to other countries [44].

Terrorism is a broad term and consisting of a highly diverse set of actions. Martha Crenshaw [45] describes terrorist acts as ranging from kidnapping of individuals to pressure governments to comply with their political demands indiscriminate bombings. However, the term is often political in nature and subjective, often stretching

the definition of the term to new areas (e.g., "cyberterrorism," "ecoterrorism,") to elicit emotions [45].

There are limited studies in computer science literature that seek to understand terrorism, radicalization, and extremism in Computer Science literature; it is often looking for signals of attacks to prevent these attacks, rather than looking at understanding what might cause an individual to radicalize [46, 47, 48]. There have been studies that have used psychological assessments, such as the HCR-20V3 [49], as the basis for building tools to identify the risks of radicalization using social media platforms [50, 51, 52, 53, 54]. Many of these tools focus on identifies risk factors, 
such as socioeconomic and demographic conditions of individuals [51], and attempt to build automated tools to identify those risk factors. These authors have also proposed analyzing and studying the relationships between users to measure the risk for radicalization.

Psychology has explored radicalization, terrorism, and extremism beyond just looking for economic and demographic factors. Instead, the field has looked at clinical or personality traits, cognitive factors, and emotions to understand what might cause someone to radicalize. Along with this, there is a rich history of studies that look at violence and the psychological traits. Computer Scientists often overlook these studies, but they can help add additional context for understanding why an individual might radicalize or even determine how de-escalation might occur for the individual.

Milan Obaidi et al. [55] identified that much of the prior research in the area of violent extremism has focused on explanations using clinical dispositions. Some psychologists have indicated a belief that group dynamics, especially in-group dynamics, may play a more significant role in violent tendencies over pathologies, personality, education, income, or any other demographic factor [56, 57]. Others have looked at psycho-pathological qualities, such as suicidal motives or mental health problems [58, 59]. The New York Police Department (NYPD) has utilized its models to help identify radicalization, focusing on "demographic, social, and psychological factors that make the individuals more vulnerable to the radical message." [60]

Sageman [61] suggested that it was personality traits that can predispose individuals towards the path of Jihad. Many early studies on the psychology of terrorism focusing on personality traits portrayed terrorists as having psychological problems [62, 63]. More recent studies have looked at terrorism through the lens of personality traits. Obaidi et al. [55] have shown that non-clinical personality traits, using the HEXACO model, may correlate more highly with tendencies towards violent extremism, mainly appearing in those that are more dogmatic, less empathetic, and less 
emotional.

Extremism also includes non-violent extremism, including political extremism and socio-political attitudes. Using the HEXACO model, it 64] shows that HonestyHumility negatively correlated with Social Dominance Orientation, and Openness to Experiences negatively correlated with Right-Wing Authoritarianism. In both cases, the correlations show that those who tend towards extremist views tend to lack humility, reflecting a hierarchical preference (one person ruling over others) in relations.

A separate study [65] using the Linguistic Inquiry and Word Count package and IBM Watson's Personality Insights found that both Left-Wing Extremists and RightWing Extremists tend to be less agreeable, less neurotic, and more open than nonextremists. Despite these correlations, Bell et al. [66] have shown that personality tests alone cannot distinguish those who would commit militant terrorist acts from non-terrorists. Another study looking at adolescents and extremism found that personality factors such as low intellect/Imagination, low Extraversion, and high Agreeableness indicate potential vulnerabilities to extremist ideologies. [67]

[65] also looked at emotions and whether they could be used to determine political extremism. It found that Left-Wing Extremists tend towards negative emotion words, while Right-Wing Extremists tend toward positive emotion words. However, the authors did note that these affective differences reflect the ideological direction of political extremists, but not their militancy.

While personality may help to add context, Other psychological models have also been applied, such as the Dark Triad / Dark Tetrad, looking at how they might identify the radicalization of extremists. The Dark Triad consists of Psychopathic, Narcissistic, and Machiavellian traits; the Dark Tetrad adds a fourth category for Sadistic traits. One study showed that this Dark Tetrad of traits could indicate radicalized behaviors in women, showing that a significant portion of non-clinical 
french college women are at risk of religious radicalization [68].

We see that while Computer Scientists have focused on identifying existing radicals and extremists, psychology has worked to develop new models in understanding the process of radicalization. Martha Crenshaw [45] highlights that research into terrorism needs to go beyond the focus on current events or speculations about the future developments of the phenomena over time. Researchers can and should continue to focus on understanding the basis for rationalizing terrorism, but psychological research can also help in understanding what causes groups to end violence [45]. While the tasks that computer scientists have focused on are no less important tasks, using Machine Learning techniques to measure various psychological traits could help in more substantial studies to understand those people that might become militant extremists, perhaps helping to prevent radicalization or deescalate extremists in the future.

\section{Deanonymization of Threats}

Methods for computer-mediated communications have increased in popularity since the 1990s with the invention of the World-Wide Web. This increase in the use of computer-mediated communications has given rise to new Social Media platforms that play a large role in economies around the world. In 2010, the Canadian Library of Parliament claimed that Social media had changed the way content is both created and consumed, and because of that, the platforms have changed the information and communications technologies sectors [69]. Between 2002 and 2011, the economic growth in Canada related to Social Media platforms grew at twice the rate of Canada's overall economy [69]. More recently, the use of social media has begun impacting presidential elections, allowing the spread of information more broadly, and creating new concerns around the dissemination of misinformation and "fake news." [70]

Anonymity on the internet allows for the creation, updating, and distribution of 
content to people around the world. This anonymity is essential in democratizing information by encouraging active engagement, particularly from members who may feel threatened by sharing information [71]. While this anonymity is vital in ensuring the privacy of those sharing information, anonymity can also create an environment that allows for much of the extant negative behaviors online [72]. These negative behaviors have real-world impacts, often affecting the lives of victims of attacks. The concept of "doxing" - the internet practice of researching and publicly broadcasting personal information [73] - and "swatting" - deceiving emergency services into sending police response teams to an individual's address [74] - have threatened the lives of people and on occasion caused unnecessary deaths. Other forms of cyberbullying have a significant psychological and social impact on both the victims and the bullies, and is a contributing factor for depression and suicide of victims [75]. More extreme forms of protecting identities online, in what is known as the "Dark Web," has allowed for the spread of hate speech, terrorism planning, and the exploitation of children [76].

Much of these harmful acts attribute to the ease with which individuals can commit acts of online deception, often aided by limited strategies to verify information conveyed to people [15]. In cases of anonymity, this seems to fit under the concept of identity deception - the willful intent to provide false information due to a lack of verification methods [77]. This form of deception can range from seemingly innocuous misrepresentations of gender, age, ethnicity, or physical appearance for online dating profiles [16] to more extreme cases, such as child pornography, sexual predators, and pedophiles [78].

While personality itself is not a good indicator of an individual's identity, it can help in understanding various potential aspects of one's identity. Things such as an individual's political leanings have correlated with personality - conservatives tend to be higher in Conscientiousness, but lower in Openness to Experiences than liberal counterparts $[79,80]$. Another benefit is the use of the constancy of personality traits 
from adolescence to adulthood [20]. This constancy can help validate the person one is talking to has remained the same throughout the communication.

Stylometry is a technique of analysis of documents used to determine the authorship of given documents. While this can be traced back to some of the early attempts to identify the authorship of Shakespearean plays, it was the work of Frederick Mosteller and David Wallace in the 1960s that laid the foundation for computer drive stylometry [81, 82, 83]. Enhancements in communications devices, including the internet, has created new challenges in the field allowing the techniques to assist in countering identity deception problems, such as email misuse [84], continuous, active authentication [85], presidential speechwriters [86], deceptive social media postings [87], and text messages from the short messaging service (SMS) [88]. Researchers have defined author stylometry as a behavioral biometric since authors can change their styles according to their desires, the genre, the topic, the author's emotional state, and a variety of other factors [89]. For this reason, various psychological models might also help identify the authorship of a document, post, or SMS.

Because of the impact of psychology on these various economic and social areas of interest, this dissertation explores a handful of behavioral models, including Emotional Intensity Analysis, Estimation of Personality traits, and Authorship stylometry. Many of these threats are not inherently in English; therefore, the impact of translation on psychological estimation systems needs to be analyzed. Chapter III highlights the models that were developed and conduct a literature review that looks at similar work in the three domains. Chapter II and IV] explores the datasets used, the translation engine that chosen for experimentation, and conducts a statistical analysis to determine the impacts of translation on the systems, and potential methods for increasing the metrics of the systems while working in various languages. 


\section{Privacy and Legal Issues}

While tools developed for identification of potential radicalization, insider threats, or the deanonymization of dangerous posting on the dark web or social media may be well-intentioned, there are potential privacy and legal concerns that might prevent the broad application of psychological tools. While companies may desire to log, monitor, and audit employee interactions on company devices, Carly Huth [90] highlights the challenges for companies establishing these policies on work devices, especially when companies may have vague personal-use policies. In these cases, these monitoring tools may lead to legal issues when used for employee termination. Carly Huth [90] gives an excellent survey of the various laws and regulations that are in place to maintain the privacy of employees and the further demands of government employers.

U.S. law enforcement and U.S. federal government agencies also have to abide by the constitutional and legal rights afforded to citizens and legal permanent residents. Many of the tools discussed may be most beneficial when used very broadly, but the Fourth Amendment of the U.S. Constitution's Bill of Rights protects people from unreasonable searches and seizures; this amendment should prevent tools such as these from being applied very broadly. While this protects U.S. citizens from many potential abuses, the "Uniting and Strengthening America by Providing Appropriate Tools Required to Intercept and Obstruct Terrorism Act of 2001" (USA PATRIOT Act) modified various laws allowing federal agencies access to various tools to combat terrorism through the use of electronic surveillance [91]. Even under this congressional act, Presidential Executive Order 12333 provides further limitations on various federal agencies that might attempt to use such tools broadly [92].

Other countries may have more relaxed legal environments allowing them to use such tools more broadly. While these types of tools may be beneficial applied broadly, companies and governments ought to approach the use of such tools sparingly to avoid potential abuses. Understanding someone's personality alone would not make them 
a potential terrorist. Understanding that an individual is emotionally angry does not make them a potential insider threat. These tools help provide additional context about a person and help inform analysts and law enforcement officers of the way an individual might react. The tools can help to understand what might have motivated the individual to commit an act. However, users of these tools should never make decisions about a specific individual without the additional context needed to make a final decision. 


\section{CHAPTER II}

\section{DATASETS}

One of the limiting factors in the exploration of this field of study is in the creation of properly ground-truthed datasets. Computational Behavioral Analytics datasets can be created through a variety of different means depending on the task. In some cases, the exploration of these types of datasets was cost-prohibitive in this dissertation. Another complicating factor in this dissertation was in finding datasets in various foreign languages. Here we explore methods used in the creation of datasets for Affect (or Emotion) analysis; a discussion of the dataset used for personality trait estimation, along with the limitations in this dissertation that prevented the creation of a foreign language dataset with the potential methodology for other scientists to conduct future studies in foreign languages; and the datasets used in Authorship Attribution.

While there are several different possible tasks under Computational Behavioral Analytics, we focus on three specific tasks in this dissertation in order to show methods for conducting these psycho-linguistic assessments that makeup Computational Behavioral Analytics and could have some potential benefit in various areas of understanding the person behind the text.

The first task is affect (or Emotion) analysis. Unlike Sentiment Analysis, affect analysis attempts to understand the intensities of the primary six emotions being ex-

pressed in the writing sample, regardless of the topic discussed in the text. When used 
in combination with topic analysis and sentiment analysis, this tool can potentially identify threats before a malicious actor can take escalating action.

The second task is personality trait estimation, or rather the exploration of estimating the traits that make up the Five-Factor Model - Openness to new ideas, Conscientiousness, Agreeableness, Extroversion, and Neuroticism (or Emotional Stability). While not necessarily indicative of whether someone will commit a malicious act (intentionally or unintentionally), it can give a better understanding of what might motivate a person and how this person might react in various situations. Other traits such as the Dark Triad or Locus of Control would be better indicators of whether someone might act maliciously.

The third task explored is Authorship Attribution. This task is a behavioral biometric, and while it is often not explored in psychology, it does rely on the author's emotional states, the author's personality, and other traits that can influence the author's word choice. This area of study has more impact in the identity sciences realm, and can be useful in identifying potential cyber-personae.

\section{Affect Analysis}

The primary purpose of Affect Analysis is to estimate the emotional intensities expressed in short textual messages, including SMS messaging or Tweets. This type of analysis is useful in estimating the emotional state of individuals who might commit malicious acts. The same analysis, when scaled up, can measure the general mood of a region as a possible indicator for social unrest within regions or groups of people, though further experimentation is needed to ensure that this marker is, in fact, useful.

Sentiment Analysis is a tangential task that is often better studied in literature than affect analysis. Sentiment Analysis generally focuses on the estimation of positive or negative valence toward specific topics, whereas affect analysis generally focuses on the emotional intensities expressed in the text (i.e., how angry is this per- 
son overall) [93]. Many studies have used lexicons, whether manually generated or generated with the help of machine learning, for the development of their systems [94, 95, 96, 97, 98. Other studies have also looked into lower-level semantic and syntactic structures, utilizing word n-grams [99] or parts-of-speech n-grams [100]. The use of lexicons remains one of the most common techniques for sentiment and affect analysis, even among recent studies looking at foreign languages [101]. Abbasi et al. [102] explored the use of many features within the linguistic space to estimate emotional intensity within short text samples.

Scientists have studied emotions for nearly a century in literature. Early studies of emotions looked at the physiological reaction of emotions [103]. This theory was later expanded on by Cannon and Bard, attempting to explain the physiological reactions as a concurrent signal being sent to the brain to evoke both an emotional and physiological reaction [104]. Further cognitive studies took on the Two-Factor Theory of Emotions proposed by Schachter and Singer [105]. This theory proposes a physiological arousal occurs first, followed by the individual reasoning about the cause of the arousal to experience an emotion.

While these theories attempt to explain emotions as an evolutionary process, various appraisal theories have also attempted to explain emotions. Appraisal theories explain the process of emotions as linked to the immediate evaluation of the individual's circumstances [106, 107, 108, 109]. This model explains the variations of emotions that an individual experiences under similar circumstances. While an individual may become saddened by the failure of a school assignment, another individual might experience anger or fear in a similar circumstance.

Psychological literature often cites two prominent theories of emotions: the emotional aspects of Plutchik's theory of actions as an adaptive problem [110, 111], and the basic emotions described by Paul Ekman [112, 113]. We utilize aspects of both of these theories for the development of the algorithm used in experimentation. Plutchik 
identifies eight basic emotions: joy, trust, fear, surprise, sadness, anticipation, anger, and disgust. Paul Ekman initially identifies six basic emotions: anger, disgust, fear, sadness, happiness, and surprise.

Paul Ekman's basic emotions were determined by looking at facial expressions to identify potential deceit from individuals. The basic emotions are anger, happiness, surprise, disgust, sadness, and fear. Expression of these emotions occur through similar facial muscular movements across both Eastern and Western cultures [114]. Later studies by Paul Ekman determined that other universal emotions exist crossculturally, though they are not always identifiable through facial expressions. These emotions include: Amusement, Contempt, Contentment, Guilt, Relief, Shame, and others [112, 115].

Plutchik's wheel of emotions, shown in Figure1a, shows each of the emotions that are identified by Plutchik. The emotions without colors shown between the colored, basic emotions are combinations of emotions. Plutchik theorizes that remorse is a combination of both disgust and sadness; contempt is a combination of anger and disgust; and so forth. Plutchik further expands this concept by looking at dyads of emotions, an image shown in Figure 1b. Plutchik defines some emotions as being opposites of one another - fear is the opposite of anger; sadness is the opposite of joy; and so forth. Each emotion that is shown 180 degrees around the wheel shows each of the opposite emotions. In Plutchik's Dyads, these opposites never occur in combination. Every other emotion is connected to highlight more complex emotions: Outrage is a combination of Anger and Surprise; Guilt is a combination of Joy and Fear; and so forth. Since emotions are often complicated, and it is often difficult to determine when a feeling is a combination of two or more feelings.

Plutchik also introduces the concept of emotional intensity. As each of the basic emotions becomes increasingly closer to the center of the wheel, the emotions become more intense: anger becomes the emotion of rage, fear becomes terror, joy 


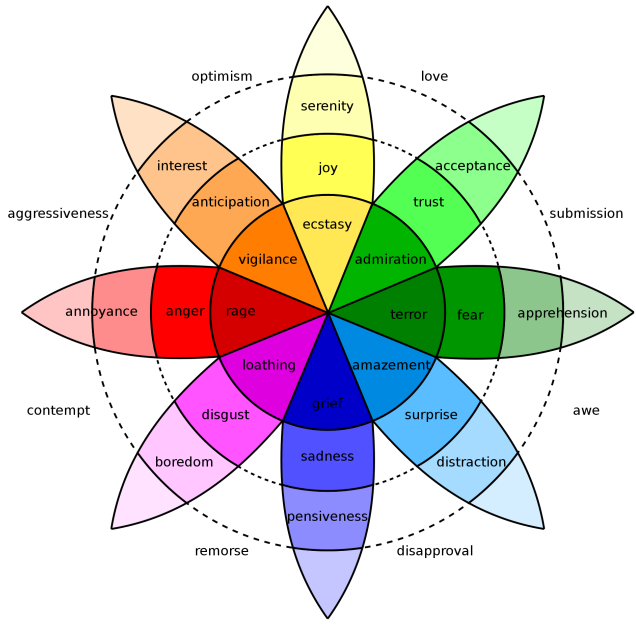

(a) Plutchik's Emotions

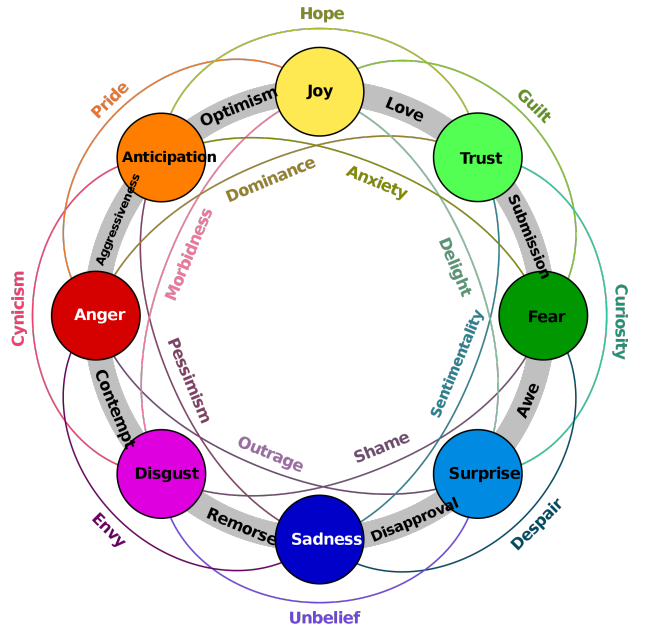

(b) Plutchik's Dyads

Figure 1. Diagrams showing Plutchik's Wheel of Emotions (a) and emotional dyads (b) that were proposed by Plutchik [110, 111].

Image (a) is in the public domain [116] and Image (b) was used under Creative Commons Attribution-Share Alike 4.0 International License [117; No modifications to the images were made. Both images were downloaded from Wikimedia Commons.

becomes ecstasy. As the emotions move away from the center, the emotions become less intense: less intense anger is annoyance, trust becomes acceptance, anticipation becomes interest. The algorithm discussed utilizes some aspects of Plutchik's theory. While the algorithm is not explicitly trying to identify specific combinations of emotions, the algorithm does allow for the possibility of multiple emotions conveyed in text. The algorithm also incorporates the concept of emotional intensities, allowing the algorithm to determine the intensity of each emotion's expression. For simplicity in creating an Arabic dataset, we utilize the emotions described by Ekman and incorporate the concept of intensities of emotions on a normalized scale.

\section{English Language Datasets}

The Support Vector Regression Correlation Ensemble methodology explored by Abbasi et al. [102, 46] appears to be one of the most flexible algorithms for understanding the intensities of emotions present in a text. For that reason, this dissertation repli- 
cates portions of their work and expanded on to determine the viability of use in foreign languages.

For the research from Abbasi and Chen [46, 102, 93], two dark web forums, AlFirdaws and Montada, were selected for exploration. For their studies, a subset of the collected forum posts were examined and coded by a domain expert. Five hundred sentences from each forum were selected and scored on a continuous, normalized scale by experts for the intensities of sentiments and affects expressed. The affects annotated in the dataset included: violence, anger, hate, and racism [93]. This dataset results in a relatively small dataset for each potential affect, and the selection by an expert could potentially bias a dataset. However, the methodology of their algorithm is potentially interesting. This dataset did not appear to be available to other researchers, so other datasets were obtained to test comparable systems.

To build the algorithm, we identified two English language datasets for testing the emotion analysis system. SemEval (Semantic Evaluation) is a competition to evaluate computational semantic analysis tools and algorithms hosted by the Associate for Computational Linguistics. In both 2007 and 2018, SemEval explored the evaluation of emotional intensities in both headlines from news sources (2007 Task 14) and Twitter posts (2018 Task 1). Both of these datasets have limitations that are not ideal in developing the final product, but they are sufficient for the needs of building and testing the algorithms in a language that could be understood by the author of this dissertation.

SemEval 2007 Task 14 is composed of news headlines from the New York Times, CNN, BCC News, and other news sources from the Google News search engine. The dataset was annotated from an online tool that displayed single headlines with six slides for each of the emotions, along with a seventh slide bar for valence. This system allowed annotators to score these headlines between $[0,100]$, where 0 is the emotion not being present in the headline, and 100 being the maximum intensity 
of the emotion. The valence score was annotated on a $[-100,100]$ scale, where 100 is a highly negative news headline and 100 was a highly positive headline [118]. This study was one of the first datasets found where the authors explore fine-grained details on emotional intensities. Before this, many studies simply relied on binary decisions of the emotion being present or not being present. Fine-grained details allow the authors to capture joint emotions that might be present in the headlines. Further analysis of their dataset is found in [118]. This study ignored the valence scores and reformatted the data into a Comma Separated Value format, containing the news headline and the annotated emotional intensities from the SemEval dataset. The emotions captured in this dataset follow the Ekman six emotions, and since all emotions align with the headline, the correlation ensemble outlined by Abbasi et al. [102, 46] can be explored with this dataset.

The creation of the SemEval 2018 Task 1 dataset is a bit more complicated. In this, the authors created a lexicon of words for each of the four emotional categories explored (anger, fear, joy, sadness). Using this lexicon, the authors polled Twitter's feed for two months to identify tweets containing words in the lexicon. From this, 1,400 tweets were randomly chosen from the joy category and annotated through a crowd-sourcing platform. For the remaining three categories that the authors call "negative emotions", 600 tweets (200 from each of the emotional categories) were randomly chosen to be annotated for all three of the emotional categories. Another 800 tweets were selected for additional annotation for each of the emotions. Because of this, each emotional category contains 1,400 tweets. Except for the 600 "negative emotion" tweets, each tweet was annotated for a single emotion [119]. The capturing of only single emotions limits the ability to use this algorithm to create correlation ensembles. Each tweet is simply binned into their emotional category with an intensity score, allowing for a regression analysis of the text to estimate the intensity of that emotional category. 
There are limitations that system architects need to be aware of when using these datasets for any specific purpose. While the SemEval 2007 Task 14 dataset is perhaps one of the more robust in capturing all six of the emotions for each headline, the use of news headlines limits the ability to use this outside of this category of text. Experimenting with angry tweets discussing topics such as "death" and "killing," it was found that news headlines rarely evoked anger. Instead, these words often were intended to evoke the emotion of sadness.

The lack of annotation of tweets for all emotions in the SemEval 2018 Task 1 prevents the use of correlations between emotions to improve the system's accuracy across these hidden correlations. This dataset makes many assumptions in its creation, and the use of a lexicon limits the potential to capture hidden features that might indicate a specific emotion that the author may not have taken into consideration.

Ideally, a future English language dataset would be collected to fix the limitations of these current datasets, but this is outside of this dissertation's scope. These limitations do not prevent the development of the algorithms for testing in foreign languages (which is the primary focus of this dissertation.) Instead, the SemEval 2018 Task 1 dataset allows for a Support Vector Machine for Regression (SVR) for each of the emotional categories explored, while the SemEval 2007 Task 14 dataset assists in building both the SVRs and in testing the correlation ensemble.

\section{Foreign Language Dataset}

A foreign language dataset is needed to test the efficacy of translations on the algorithms. Unfortunately, many of the currently existing datasets only focus on English. A dataset was created in order to test the efficacy of translations in emotion analysis. For our experimentation, we follow a similar data structure as the SemEval 2007 challenge dataset, choosing to use the Ekman basic emotions (anger, disgust, sadness, 
fear, happiness, and surprise) [118]. These emotions provided a good baseline in experimentation and seemed to provide the most culturally agnostic set of emotions for building such a dataset.

The first task in building the foreign language dataset was the collection of, in this case, Arabic language tweets. While Modern Standard Arabic (MSA) is a standardized literary Arabic used in formal writings, the use of twitter often entails more colloquial terminology, though the Arabic script remains the same. The Egyptian Arabic dialect was chosen as a focus to account for potential colloquialisms that might occur in social media postings. A dataset was created using the $1 \%$ feed using Twitter's API and further filtered to account for the target language using Twitter's language tagging algorithm. We then looked at the geotag for tweets around a target area (in this case, geotags from Egypt) to maximize the collection in the targeted Egyptian Arabic dialect. All tweets were post-processed for anonymization, removing proper names and identifying information using Python's NLTK libraries.

Once the approximate threshold of 75,000 tweets was achieved, a series of three cascading tasks were given to annotators on the Amazon Mechanical Turk (AMT) platform. An initial test was given to potential annotators to ensure that the annotators spoke the Egyptian dialect of Arabic well enough to understand the presented tweets. Even using this method of identifying Egyptian dialect speakers, the method is far from perfect. There are significant overlaps between different varieties of Arabic, and many (though not all) of the dialects are mutually intelligible. Further complications arise because many Arabic speakers also have at least a passing familiarity with the Egyptian dialect, since Egypt can arguably be called the entertainment capital of the Arabic-speaking world.

Despite the challenges, the creation of this dataset used a test created by linguists and outlined in a technical report [120]. Annotators were given a list of Arabic sentences containing colloquial terms and phrases that were not highly intelligible in 


\begin{tabular}{|l|l|}
\hline TASK \# & Median Agreement \\
\hline Task 1: Is there emotion? & $91 \%$ \\
Task 2: Is there (specific emotion? & $94 \%$ \\
\hline
\end{tabular}

Table 1. Median Agreement between Annotators during tasks 1 and 2.

other Arabic dialects. The initial test asked the annotators to translate the Arabic sentence to mimic the task closely. While the task is slightly more challenging than a reading comprehension test, it is closer to the intended Human Intelligence Task (HIT) on AMT. An example test question is in Figure 2.

Once an annotator was approved for conducting the tests, a series of cascading tasks would occur. During the first two tasks, a series of control questions measured the agreement between annotators. Table 1 lists the median agreement between annotators on all control items in tasks 1 and 2 .

The first task verified that the tweet shown was, in fact, the correct dialect, and whether the tweet expressed any emotions. Annotators read the tweet and indicated one of the following: Tweet expresses an emotion; Tweet contains no emotion; Cannot tell if the tweet contains an emotion; or that the tweet contains some language or dialect other than Egyptian Arabic.

In the instructions to the annotators, annotators were told to use the "wrong dialect" liberally. Annotators marked the tweet as the wrong dialect even if an emotion was present; if there was a mix of languages in the tweet (English and

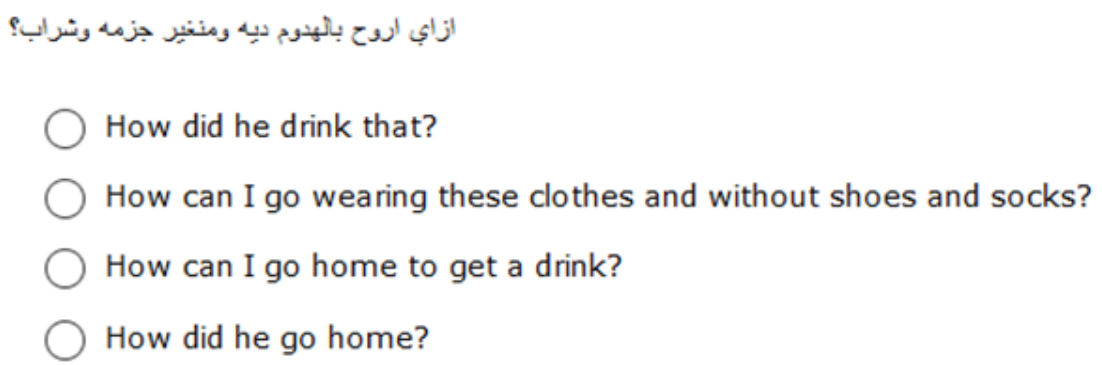

Figure 2. Example test question used for qualification to participate in annotation of the Egyptian Arabic dataset. 
Arabic); or anything written in MSA or formal literary Arabic, such as passages from the Qur'an or Bible, formal invocations, prayers, greetings, or rituals. When a tweet was the "wrong dialect", the tweets were not evaluated further, and the tweets were excluded in the dataset. The annotators had to decide whether the tweet contained an emotion even if the tweets were too short to determine the dialect. Any loan words or Latin-scripted twitter handles or hashtags were not marked as wrong dialects either.

Once these tasks were completed, any tweets of the wrong dialect or expressed no emotions based on annotators consensus, were discarded from the dataset. All of the remaining tweets that contained some emotion were passed on to the second task. In this second task, tweets were bucketed based on the emotions that annotators believed that tweets contain. The annotators were shown a tweet and asked whether the tweet contained a specific emotion (e.g., anger) by selecting one of the following options: Has anger, No anger, Cannot tell, and not Egyptian Arabic. The option "Not Egyptian Arabic" remains in the task if any tweets managed to slip through the previous filters, though this option was infrequently selected after task 1.

Each tweet was shown for the six emotions, and to at least five annotators for consensus on each emotion. Therefore, each tweet was shown at least thirty times - five times for anger, five for happiness, continuing for each emotion. Since each tweet was tested for each emotion, a tweet was allowed to be binned into multiple emotional categories. These multiple categories of emotions allow the algorithms to identify correlations between emotions that might not otherwise be captured, further improving results and allowing experimentation against the algorithm(s) described in Abbasi et al. [102, 46].

The final task was the task intended to estimate relative intensities of emotions. For this task, minimization of cognitively demanding tasks was desired, such as rating how intense an emotion was using Likert scales. Using cognitively challenging tasks can result in more significant disagreements between annotators since each would cog- 
nitively anchor their responses based on individual experiences. These disagreements can become problematic when training an algorithm, especially on subjective targets such as the emotional intensities explored in the study.

Instead, a simple pairwise judgment task was given to annotators for measuring the intensities. For each of the emotional buckets, two tweets were selected; the annotators were asked for comparative judgments on which of the tweets contained the most intense emotion. While, ideally, every tweet would be compared against one another, this would increase the costs and require significant amounts of time for annotators to complete. Instead, each tweet was involved in at least ten comparisons using random selections. Using these comparisons, we were able to induce the emotional intensities from the pairwise judgments using linear programming.

For this process, we define a set of tweets as the tweets from a specific emotion $(A)$ :

$$
\text { tweets }=\left\{t_{1}, t_{2}, \ldots, t_{n}\right\}
$$

$f_{a}\left(t_{i}\right)$ is defined as the function that determines the intensity of the emotion $(A)$ from tweet $\left(t_{i}\right)$. In order to create a linear programming optimization problem, we assume that we would like to computer $f(t)$ such that we respect as many of the annotator's judgements involved each tweet as possible, and where we assume " $t_{1}$ is angrier than $t_{2}$ " implies $f_{\text {anger }}\left(t_{1}\right)>f_{\text {anger }}\left(t_{2}\right)$. Using these assumptions, we can encode the judgements as an inequality constraint:

$$
\begin{gathered}
f_{A}\left(t_{1}\right)>f_{A}\left(t_{2}\right)+1-\lambda_{1} \\
f_{A}\left(t_{3}\right)>f_{A}\left(t_{2}\right)+1-\lambda_{2} \\
\text { etc. }
\end{gathered}
$$

Where $\lambda \mathrm{s}$ are slack variables that can be increased or decreased to account for inconsistent judgments, the linear programming task becomes a minimization of the 

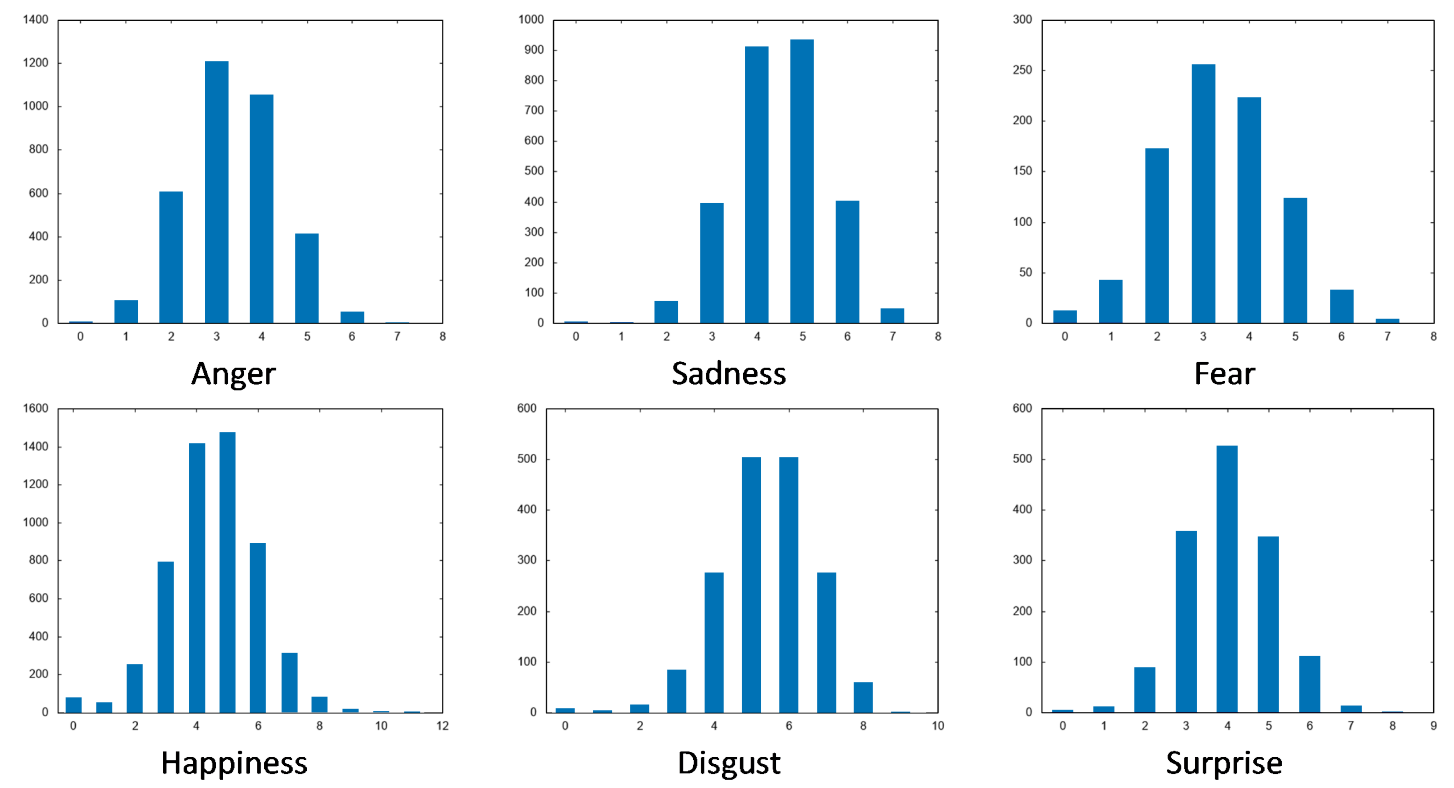

Figure 3. Equivalency classes for intensity scores for each of the six emotions.

\begin{tabular}{l|rr} 
Emotions & Tweets & Yield (\%) \\
\hline All tweets & 75,019 & - \\
Any emotions & 28,165 & $38 \%$ \\
Anger & 3,440 & $12 \%$ \\
Sadness & 2,766 & $10 \%$ \\
Fear & 870 & $3 \%$ \\
Happiness & 5,388 & $19 \%$ \\
Disgust & 1,727 & $6 \%$ \\
Surprise & 1,461 & $5 \%$
\end{tabular}

Table 2. All rows show the total number of Egyptian Arabic tweets collected for that class; "Any emotions" shows the filtering results in the first Task, the "Any emotions" yield shows the percentage based on "All tweets"; Each of the emotions list the total number of tweets in that emotional class, the yield percentage is based on the "Any emotions" tweets. 
sum of lambdas $\left(\min \sum \lambda\right)$, or minimization of the total effect of the inconsistencies. Equivalency classes for intensity scores were induced for each of the six emotions using this method. A histogram showing the number of tweets is in figure 3 .

The final Arabic corpus collected contained 3,440 anger tweets; 2,766 sadness tweets; 870 fear tweets; 5,388 happiness tweets; 1,277 disgust tweets; and 1,461 surprise tweets. Of the initial $75 \mathrm{k}$ Egyptian Arabic tweet dataset, only about $38 \%$ of the tweets contained any emotions. Within those, only a small percentage yielded tweets with the targeted emotions. The yield percentages and fine yield totals are summed up in table 2 .

Only the tweet text was kept in the collection, and no other demographic information was kept during the collection. This process was followed for the anonymity of the users. Since this was a random sampling of twitter users, we expect that the demographics should be similar to those of the usual twitter users.

\section{Personality Trait Estimation}

Datasets that are relevant to any psychological assessment of users are harder to acquire, whether through text, audio, or video. These datasets need to collect the media and also administer relevant psychological assessments, such as the International Personality Item Pool (IPIP), Revised NEO Personality Inventory (NEO-PIR), short dark triad (SD3), or another psychometric tool commonly employed by psychologists. While the psychological instruments and text from the users are easy to acquire individually, it is rare to find datasets that acquire both social media and psychometric instruments.

In Personality Trait Estimation, algorithms attempt to estimate each of the tested traits against a psychological personality instrument. Various instruments could help build algorithms, computational scientists and psychologists have explored the use of some instruments for various Human Resources related tasks. Some of the most 
prominent instruments include the DiSC profile assessment, Big Five Inventory, HEXACO, the Minnesota Multiphasic Personality Inventory, among others. Other psychological instruments also exist that may allow for correlations to written text or other forms of multimedia. Instruments such as the Dark Triad or Dark Tetrad might be relevant and exciting to explore, but data limitations prevented the exploration of these instruments. Many people are also familiar with tests such as the Myers-Briggs Type Indicator assessment. While this test has become a standard in some workplaces, its categories are not very reliable, not scientifically valid, not independent, and not as comprehensive as other scientifically valid experimentation [121]. Instead, this dissertation focuses on scientifically valid instruments.

Industrial psychologist Walter Clarke created the DiSC assessment, publishing his first version in 1956 [122]. Walter Clarke based his model on William Marston's earlier works that theorized that the expression of emotions categorizes into four primary types: Dominance (D), Inducement (I), Submission (S), and Compliance (C) [123]. Wiley, the test publisher, has continued developing the DiSC profiles attempting to make the tests more accessible to users of the test. Human Resources related companies have explored the use of the DiSC profile as a potential for identification of team fit, dynamics, and for hiring purposes for companies [3].

The Minnesota Multiphasic Personality Inventory (MMPI) is a more clinical test intended for testing personality and psychopathology in adults [124]. The test was created by Schielem Baker and Hathaway in 1943 and published by the University of Minnesota Press [125]. Since that time, the test has taken on several revisions, allowing the test to be given to adolescents (MMPI-A) [126], and adopting newer theoretical approaches to personality test development (MMPI-2-RF) [127]. It has been used by Psychologists and other Mental Health professionals to assist in the differential diagnosis and the development of treatment plans. While it has its basis in clinical domains, it is also often used in forensic psychology and candidate screening 
[128.

The DiSC and MMPI are instruments for consideration in further studies for correlations to multimedia, but Psychological literature commonly focuses on the use of the Five-Factor Model or the HEXACO models for personality and behavioral studies. The models came about as a result of factor analyses of both survey data and lexical features from several independent researchers. Early lexical studies in the 1940s by Cattell [129] found 16 personality traits. Tupes and Christal [130] were the first to propose just five factors in 1961, but this analysis did not catch on until independent researchers conducted similar studies through the 1980s and 1990s, including the work from Goldberg [131], and Costa and McCrae [132].

The Five-Factor Model (FFM) is a taxonomy that identifies five underlying dimensions used to describe personality:

- Openness to experience

- Conscientiousness

- Extraversion

- Agreeableness

- Neuroticism (or, when inverted, Emotional Stability)

Openness to Experience consists of two major sub-components: intellectual dispositions and the other related to aesthetic appreciation and sensory experiences [133]. McCrae and Costa [134] highlight that while Openness may be related to Intellect, it is an indicator of personality and not intellectual ability. They continue by saying that people who score high in Openness do not necessarily have a corresponding high Intelligence Quotient (IQ). Those who score highly in Openness have a higher intellectual capacity; often enjoy art, music, or other aesthetic impressions; or have 
a wide variety of interests. Those with a low openness score are often described as more conservative and often repress anxieties. [134]

Conscientiousness determines how someone takes obligations and tasks. Highly Conscientious people tend to show self-discipline, and often plan behaviors rather than act spontaneously. They are often neater and more systematic than their counterparts. Hogan [135] also views Conscientiousness as inhibiting impulsive behaviors. People who score lower on the Conscientiousness scale tend to be more laid back, less goal-oriented, and maybe more likely to engage in antisocial and criminal behaviors [136].

Extraversion tends to be representative of how outgoing or reserved a person is in situations. Watson and Clark [137] identify seven components that make up Extraversion: venturesomeness, affiliation, positive affectivity, energy, ascendance, and ambition; McCrae and Costa view of Extraversion assigns ambition to Conscientiousness [134] and breaks affiliation into two categories, warmth and gregariousness. Those who score low on Extraversion tend to be more quiet, reserved, silent, or withdrawn.

Agreeableness reflects a general concern for social harmony and tend to value getting along with others. Those who are highly agreeable tend to be more considerate, kind, generous, trustworthy, and willing to compromise their interest with other individuals [138. Those who score low on agreeableness tend to have a less optimistic view of human nature and tend to place self-interest above other individual's interests. They are often competitive, argumentative, or seen as less trustworthy [139].

Neuroticism represents the tendency to express emotional distress. Highly Neurotic scorers tend to experience negative emotions and are more prone to develop a variety of psychiatric conditions [140]. These distressful experiences are often associated with irrational thinking, low self-esteem, and ineffective coping [132]. While low Neurotic scorers are not necessarily mentally healthy, it does mean that they are 
usually even-tempered, calmer, and more relaxed than their counterparts.

The HEXACO model used the same factor analysis methods that helped in the discovery of the FFM. The HEXACO model adds a single personality trait, HonestyHumility, while renaming Neuroticism as Emotionality. The discovery of the HonestyHumility trait occurred while conducting comparable analyses in foreign languages. Analysis in foreign languages showed that a sixth trait existed. It was not until modern computing power could further analyze the English language that the sixth trait was found in English language studies. [141] The Honesty-Humility trait is associated with sincerity, fairness, pretentiousness, and greed [142].

\section{Personality Dataset}

For experimentation, and due to data limitations, studies in this dissertation focus on the FFM traits. Between 2007 and 2012, the MyPersonality datasetº was created at the University of Cambridge, collecting both social media posts and various psychological instruments [143]. This dataset was collected with the consent of its users through a Facebook application. They targeted subjects through a variety of means having each user provide permission to download demographic and status updates from the Facebook platform. Subjects provided a self-report using an implementation of both the 100- and 300-questionnaire of the IPIP proxy for NEO-PI-R, receiving a reliability reported in their respective manuals or standardization samples.

The dataset had numerous tests available for subjects to take, and not all subjects gave consent to collect their Facebook status updates. This mismatch of subjects leads to some inconsistencies in the number of viable subjects for training and testing any machine learning algorithms. When looking only at those subjects who took the

*This dataset is no longer available and was obtained by the University of Louisville before the dataset's closure for research purposes. The dataset was removed by the University of Cambridge to cooperate with changes in Facebook's Terms of Use, but the dataset remains scientifically valid. Future research into personality estimation, by this author or others, will require building comparable datasets for scientific purposes. 


\begin{tabular}{l|rrrrr} 
Statistic & Openness & Conscientiousness & Extraversion & Agreeableness & Neuroticism \\
\hline Mean & 3.79 & 3.49 & 3.58 & 3.55 & 2.77 \\
Median & 3.80 & 3.50 & 3.67 & 3.58 & 2.75 \\
Std. Dev. & 0.68 & 0.73 & 0.81 & 0.70 & 0.80 \\
Minimum & 1.00 & 1.00 & 1.00 & 1.00 & 1.00 \\
Maximum & 5.00 & 5.00 & 5.00 & 5.00 & 5.00
\end{tabular}

Table 3. Basic Statistics for the FFM traits found within the Dataset for all subjects. Numbers are normalized between $[1,5]$

NEO-PI-R for collection, we have a total of 3,029,503 total users.

For each of the FFM traits, we expect to see a normal distribution across the normalized scale from $[1,5]$. Histograms showing the number of subjects which fall within the scale is in Figure 5. Each of these traits is skewed slightly to the right (with Neuroticism being inverted, and skewed slightly to the left. The mean of each of these generally fall around 3.5 - the exceptions being Openness, which is at 3.8, and Neuroticism, at 2.7. Each of the standard deviations is between 0.68 and 0.8 on the scale. Complete statistics for the data within each trait is in Table 3 .

While the NEO-PI-R data contains over 3 million unique users, there are far fewer users who consented to having their Facebook status posts collected. In this case, there were only 153,727 unique users in the Status Updates data. This subset of users consists of over 22 Million unique status updates that were collected. By limiting the FFM Trait data to only this subset of users, we can see that the statistics do not change significantly, as shown in Table 4. This subset also does not significantly alter the distribution of the user scores, as shown in Figure 6.

\begin{tabular}{l|rrrrr} 
Statistic & Openness & Conscientiousness & Extraversion & Agreeableness & Neuroticism \\
\hline Mean & 3.85 & 3.47 & 3.52 & 3.57 & 2.73 \\
Median & 3.95 & 3.50 & 3.5 & 3.65 & 2.75 \\
Std. Dev. & 0.67 & 0.73 & 0.81 & 0.70 & 0.80 \\
Minimum & 1.00 & 1.00 & 1.00 & 1.00 & 1.00 \\
Maximum & 5.00 & 5.00 & 5.00 & 5.00 & 5.00
\end{tabular}

Table 4. Basic Statistics for the FFM traits found within the Dataset for only those users who provided status updates. Numbers are normalized between $[1,5]$ 
Further exploration in the data reveals that the number of posts for each user follows an exponential distribution, as shown in Figure 4. This data shows that the median number of posts provided by users was 93 status updates per user, the maximum for a user was 2,441 status updates. Users within this subset should provide enough data for training and testing any algorithms.

Further demographic information helped to determine if this data would be useful in identifying whether any foreign language data exists in the dataset. Much of this data was not useful as many people appeared to obfuscate information about their country. We found a total of 77 different locales that were provided by user demographic information. While en_US and en_GB were the two largest populations, the other locales had far fewer and include countries with which the data did not correspond to any foreign language data found. This mismatch of language and locale indicates that the locale is not a useful tool for identifying foreign language data. It also appears that the country that the authors had listed would not be useful in identifying potential foreign language data. The data had 218 countries represented for users in the subset, including 20 users in "Antarctica."

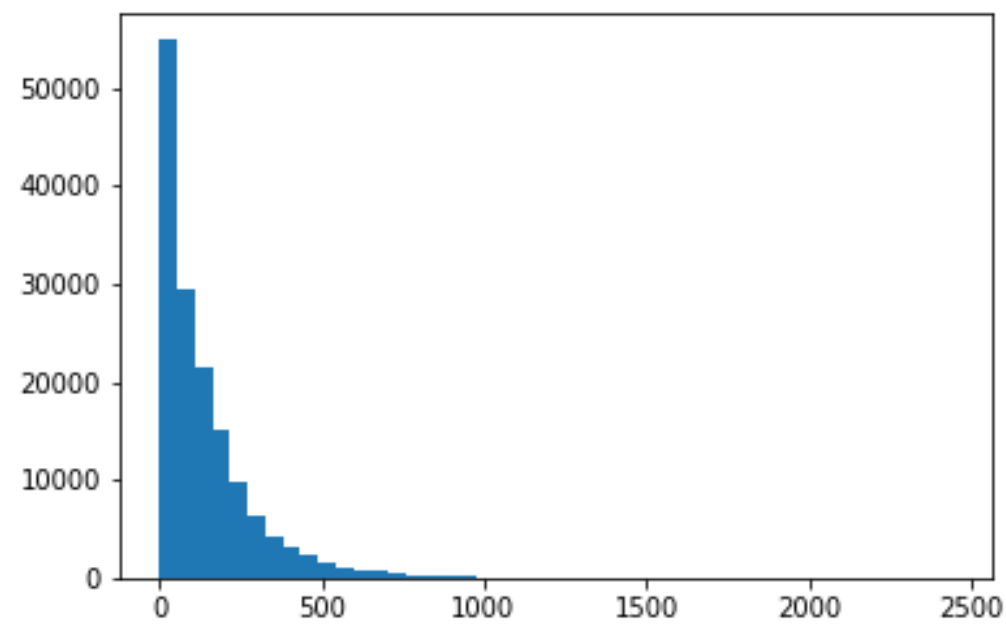

Figure 4. Normally distributed Posts per user within the subset of data. 


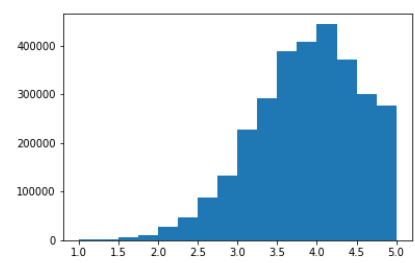

(a) Openness

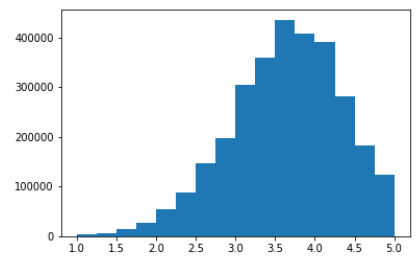

(d) Agreeableness

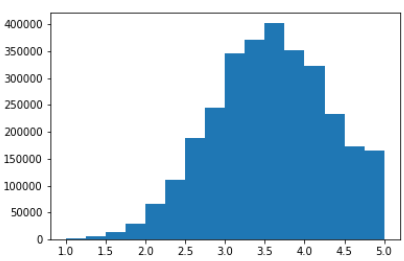

(b) Conscientiousness

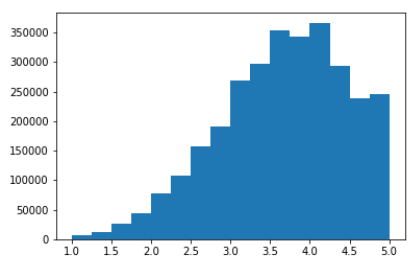

(c) Extraversion

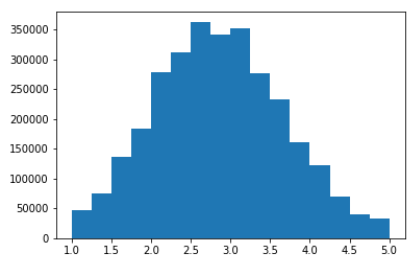

(e) Neuroticism

Figure 5. OCEAN Traits for each of the 5 personality traits for all subjects that took the personality instrument.

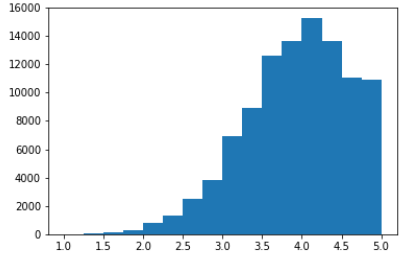

(a) Openness

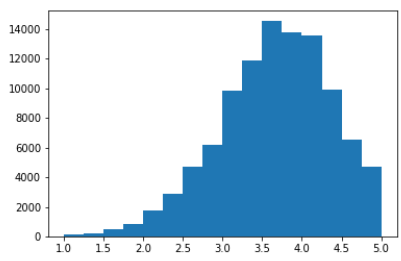

(d) Agreeableness

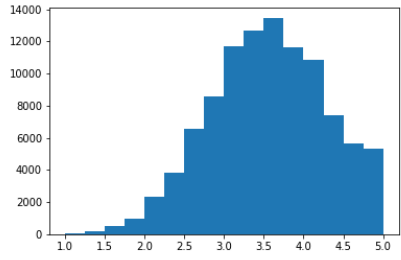

(b) Conscientiousness

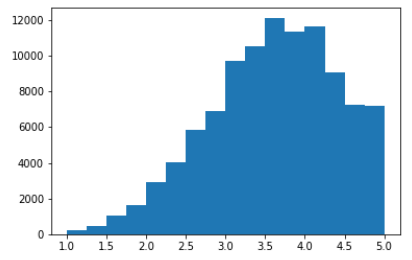

(c) Extraversion

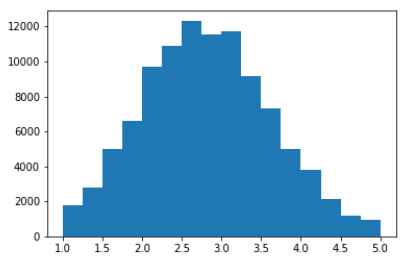

(e) Neuroticism

Figure 6. OCEAN Traits for each of the 5 personality traits for only the subset of users who have status posts. 


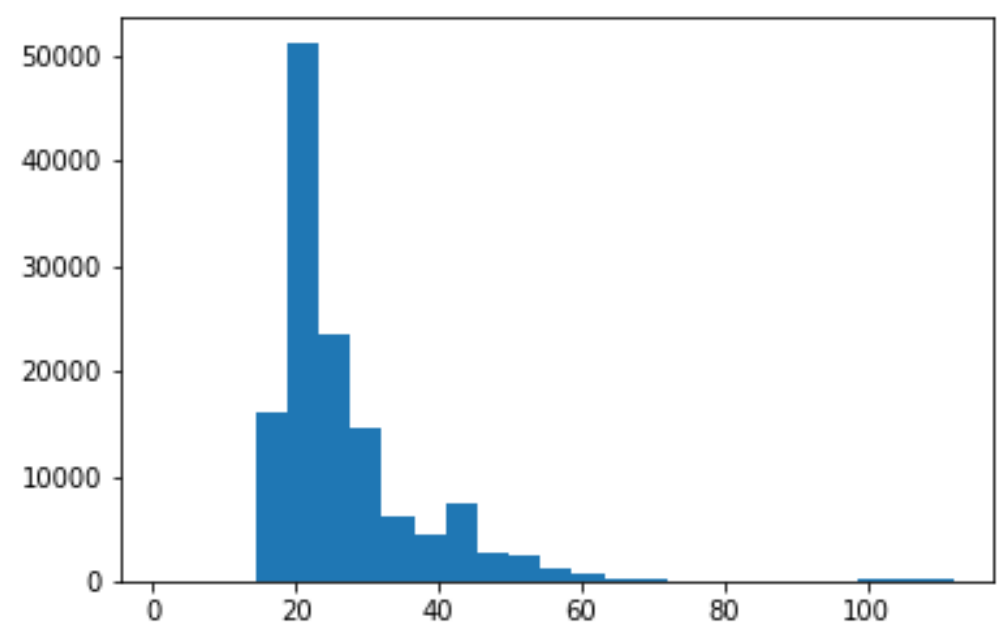

Figure 7. Normally distributed Age of users within the subset of data.

Demographic information such as gender and age helped to determine potential bias in the datasets. The data appears to have a slight over-representation of women in the dataset, containing 86,319 female subjects and 66,470 male subjects, who provided status updates. The users' age distribution appears representative of social media users, and is shown in Figure 7. The age data shows a normal distribution cut off at the age of 18 , which is expected, since children are a protected class in research studies. Most of the users in the study fall between the ages of 20 and 30. However, the collection does include older users, and may also contain noise created by users hiding their age, as some ages include users over the age of 110 .

\section{Identifying Non-English users}

From the demographic information explored in the subset of data, the demographic data would not be useful in identifying potential foreign language data in the dataset, so other methods had to be used to determine the viability of this data for experimentation. The method identified users who spoke primarily another language by looking at the text in their status updates. 


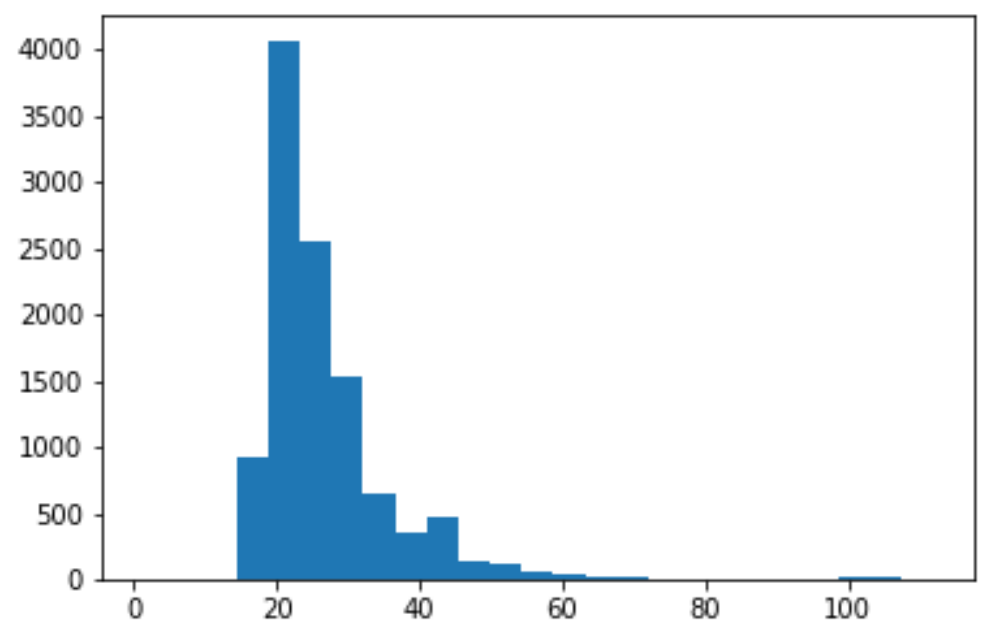

Figure 8. Normally distributed Age of users within the Foreign Language subset of data.

Every status post in the dataset was looped through so that each posts' language could be established using python's LangDetect library, a direct port of Nakatani Shuyo's language detection library for Java [144]. Going through each post in the data allowed each post's language to be determined. This library is not entirely accurate, and the potential for one-off status posts by users who may not speak the language posted, identification of foreign language data did not solely rely on the LangDetect library.

Each post was sorted to their respective authors once each post's language was determined. Each language found for the authors was summed. If most of the posts from an author was determined to be English, we assumed that the author's primary language was English. While this may remove some that might be fluent in multiple languages, we took a conservative approach during this downsizing to ensure that we identified authors with another primary language. A total of 11,829 authors were found whose primary language was not English. During this stage, a file containing each of the authors' posts was created to simplify translations in the next stage.

Further sub-sampling of authors to only those who spoke a non-English primary 


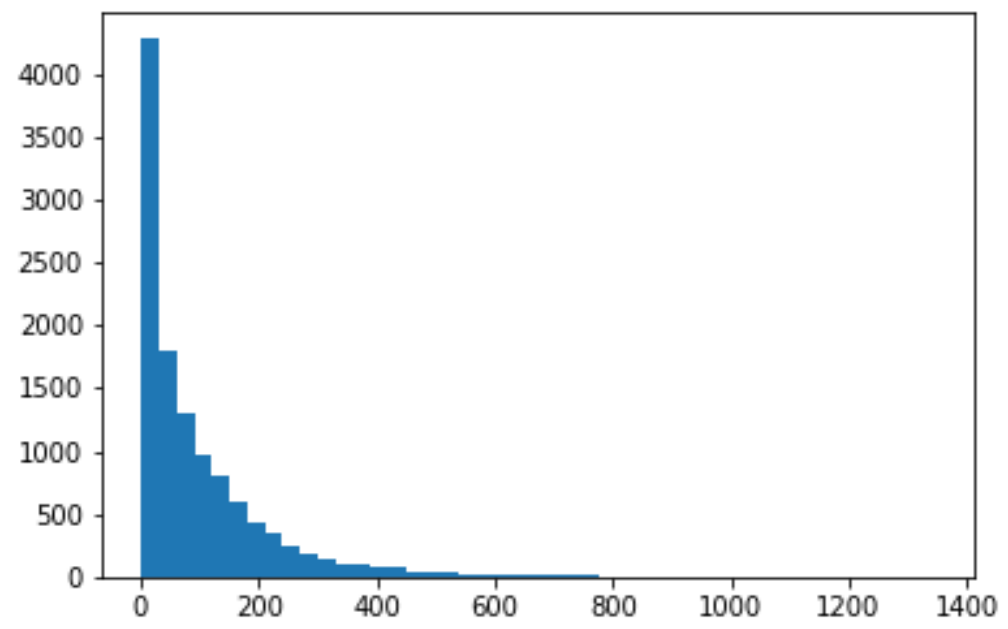

Figure 9. Normally distributed Posts per user within the Foreign Language subset of data.

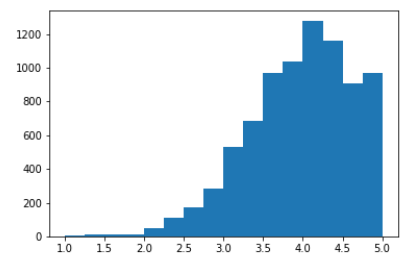

(a) Openness

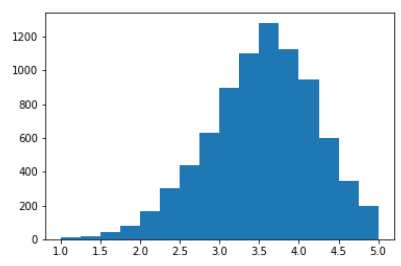

(d) Agreeableness

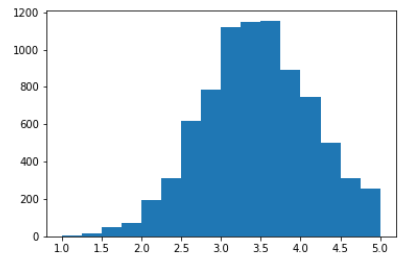

(b) Conscientiousness

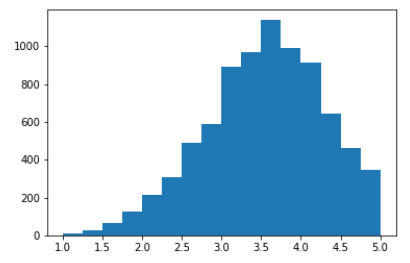

(c) Extraversion

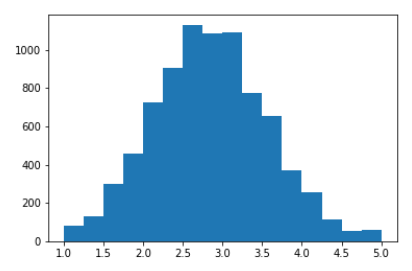

(e) Neuroticism

Figure 10. OCEAN Traits for each of the 5 personality traits for users who speak a foreign language. 


\begin{tabular}{l|rrrrr} 
Statistic & Openness & Conscientiousness & Extraversion & Agreeableness & Neuroticism \\
\hline Mean & 3.88 & 3.36 & 3.45 & 3.44 & 2.76 \\
Median & 4.00 & 3.33 & 3.5 & 3.5 & 2.75 \\
Std. Dev. & 0.67 & 0.69 & 0.75 & 0.68 & 0.72 \\
Minimum & 1.00 & 1.00 & 1.00 & 1.00 & 1.00 \\
Maximum & 5.00 & 5.00 & 5.00 & 5.00 & 5.00
\end{tabular}

Table 5. Basic Statistics for the FFM traits found within the Dataset for only those users who speak a Non-English Primary Language. Numbers are normalized between $[1,5]$

language did not significantly alter the demographics. The authors within the subset of foreign-language authors had a total of 1,214,565 status updates. The distribution of posts per user is in Figure9. Age distributions were not significantly altered within the subset either, and is in Figure 8 .

Distributions were also not significantly altered by sub-sampling to Foreign Language authors. We see the basic statistics of the FFM traits in Table 5, and the histograms for each of the five traits in Figure 10. Based on this subset, we should be able to reliably use this dataset in our algorithm to determine whether the use of translations of foreign languages will have any significant impact on the estimation of FFM traits.

\section{Authorship Attribution}

As mentioned in Chapter I, authorship attribution and verification is not necessarily a psychological trait, but is a behavioral biometric that can be informed, and may rely on various psychological traits for decisions in word choices. Thus, the exploration of authorship attribution and verification is of interest in this dissertation as well. Instead of creating new algorithms, in this task, we explore five prominent algorithms in the authorship attribution domain. These five algorithms are, notably, the bestperforming algorithms found by Neal et al. [89].

We verify the results of Neal et al. by exploring Five authorship attribution algo- 
rithms in Chapter III against the CASIS dataset. The CASIS dataset consists of 4,000 blog samples from 1,000 English speakers, totaling four samples per author. Each sample averages 13 sentences, 304 words, and 1,634 characters. North Carolina Agricultural and Technical University collected the dataset by the Center for Advanced Studies of Identity Science for authorship attribution experimentation. The dataset has helped in the testing of Authorship Attribution algorithms and the creation of tools for adversarial authorship [145, 146, 147].

\section{BOLT Datasets}

The BOLT datasets were created by the Linguistic Data Consortium for the DARPA BOLT (Broad Operational Language Translation) program [148]. This program focused on developing machine translation and information retrieval systems with informal genres, primarily user-generated content. While the BOLT datasets focus on machine translations, samples of the data show that LDC retained author usernames from the forums, making the dataset potentially useful in experimentation of authorship attribution against foreign languages.

Two of the collected datasets proved to be useful in this dissertation's experimentation, the Arabic Discussion Forums [149] and the Chinese Discussion Forums [150]. Both datasets were collected in the same manner. Native speaker annotators seeded the collection efforts by scouting web pages for specific content. Scouts sought content in a specific dialect: Egyptian Arabic for the Arabic dataset, and Mandarin Chinese in the Chinese dataset, and were attempting to find original, interactive, and informal conversations on posts. Once the scouts identified an appropriate thread, they would upload a URL and some information to a database. If multiple threads on a site were submitted, the entire forum was scrapped.

With the scouts' information, LDC harvested HTML files from each of the sources and converted them using custom scripts. Anonymization and cleaning occurred, 


\begin{tabular}{c|c|c} 
Number of Posts & Arabic User Count & Chinese User Count \\
\hline$>25$ & 53677 & 79101 \\
$>50$ & 36784 & 42568 \\
$>75$ & 28629 & 29022 \\
$>100$ & 23689 & 21803 \\
$>125$ & 20147 & 17194 \\
$>150$ & 17530 & 14072 \\
$>175$ & 15429 & 11928 \\
$>200$ & 13829 & 10264 \\
$>225$ & 12484 & 8943 \\
$>250$ & 11383 & 7887 \\
$>275$ & 10449 & 6992
\end{tabular}

Table 6. The Table shows counts of users for authors who posts more than the number of posts listed.

including attempted removal of quotes from the sources, to avoid text that might not be from the specific author. While LDC took measures to ensure that the data contains only the proper dialect and removes any quotes, the authors note that some quotes or other dialects may remain in the dataset [150, 149].

The original Arabic dataset contains 251,581 unique usernames and a total of 13,269,241 total posts across all users. Within this original dataset size, the average number of posts per user is about 52 posts with a standard deviation of 263 posts. We see in the histograms shown in Figure 11 that the distribution is exponential with a minimum of 1 post for a user and a maximum of 28,028 posts for a user. The median of this exponential distribution is 4 Posts per user. We see the histograms and medians of the distributions that users tend to fall between 1 and 10 posts per user. While this does not discount the dataset for authorship verification, it does show that much of the data is not ideal for authorship attribution or verification.

There is a sizable set of users who had a large number of posts within the dataset. The Counts of users with minimum numbers of posts are shown in Table 6. As the number of minimum posts increases, we see a drop-off in minimum users (as is expected in an exponential distribution), but there are 10,449 users with greater than 
275 posts within the BOLT Arabic dataset.

We see similar effects occur in the BOLT Chinese dataset. The original Chinese dataset contains a total of 1,331,569 unique usernames, with 15,160,154 total posts. The average posts per user is significantly smaller than the Arabic dataset at 11 posts with a standard deviation of 204 posts. As shown in Figure 12 , the distribution follows an exponential distribution with a median of 2 posts and a maximum of 191,651 posts for a single user.

The drop-off users occurs much quicker than the Arabic dataset, as shown in Table 6. The number of users with more than 25 posts has 26,000 more users than in the Arabic dataset. As we increase the number of posts, we see a sharper decline, requiring only 200 posts to get a list of 10,000 possible users. More data is collected in the BOLT Chinese dataset, but this dataset for authorship attribution or verification will require less overall data per author to be usable for the experimentation.

\section{Choosing users}

LDC provides the dataset in two formats, HTML and XML. While both are usable, the XML data provides a small size to work with while maintaining the author's original text. A script was run to loop through all of the files in each of the collections from LDC to obtain individual posts. The authors of posts were obtained from the XML files. Each post was sorted by their author to determine the number of posts each author had in the dataset. This method also allowed for the determination of the dataset's value for potential authorship attribution or verification experiments. A file was created containing posts of each author for analysis based on the outcomes of the counts of posts.

Instead of using the number of posts by the authors, it was decided that number of characters would give a better understanding of the potential useful samples in the dataset. Author files that were created were processed and sorted based on the 


\begin{tabular}{l|r|r|r|r|r|r|}
\cline { 2 - 6 } & \multicolumn{2}{|c|}{ Trans. Raw } & \multicolumn{2}{c|}{ Cleaned } & \multicolumn{2}{|c|}{ Subsets } \\
\cline { 2 - 7 } & Chinese & Arabic & Chinese & Arabic & Chinese & Arabic \\
\hline Authors & 1,206 & 2,120 & 1,173 & 2,083 & 1,003 & 492 \\
Posts (or Samples) & 903,857 & $2,179,716$ & 801,001 & $1,904,106$ & 25,075 & 12,300 \\
Avg. Words per Post & 245.86 & 58.60 & 263.13 & 64.20 & $1,904.53$ & $1,167.06$ \\
Avg. Sentences per Post & 13.97 & 2.23 & 14.88 & 2.34 & 104.82 & 40.80 \\
Avg. Posts per Author & 749.46 & $1,028.17$ & 682.87 & 900.29 & 25 & 25 \\
Avg. Word per Post per Author & 655.97 & 104.05 & 678.27 & 109.54 & & \\
Avg. Sentences per Posts per Author & 36.12 & 3.77 & 37.29 & 3.93 & & \\
\hline
\end{tabular}

Table 7. Statistics for the translated portions of the BOLT datasets for possible use in authorship attribution experimentation.

number of characters for every post of the author. From this sorted list, only the top 10,000 users were selected for potential translation. This translation phase will be described further in Chapter IV.

The translation system was run for a smaller subset of those users until more than 1,200 users completed. A more significant subset was not completed due to the length of time needed to complete the translations. Running 1,200 users took multiple weeks to complete, and running in both Chinese and Arabic took several months. Due to a desired sample size large enough to determine whether translations affected authorship attribution, we did not need to complete the translations on all 10,000 users.

After translation, and with the assistance of colleagues at the University of Florida, the translated dataset was further analyzed, cleaned, and compiled for further experimentation. Some of the data was not properly translated because of the method of translation that was used. These users were thrown out. Within the dataset, a total of 1,202 out of 1,206 translated Chinese users were found to be usable, and 2,118 out of 2,120 translated Arabic users were found to be usable. Further analysis of the translated data is in Table 7.

Further cleaning occurred on the data to remove duplicates in the original text and their corresponding indices in the translated text, any HTML and CSS style tags that remained in the datasets, any standard text or phrases, and any null posts or those 
with any foreign characters. We do see a slight improvement in the statistics because of the removal of some of the authors. These improved statistics include the average words per post, average sentences per post, average words per post per author, and average sentences per post per author. However, many of these remain low. Based on prior experimentation, a script was used to further break the dataset into 25 samples per author to allow for increased performance of the algorithms by increasing the number of sentences per sample. Statistics for both cleaning and creating the subsets is in Table 7. 


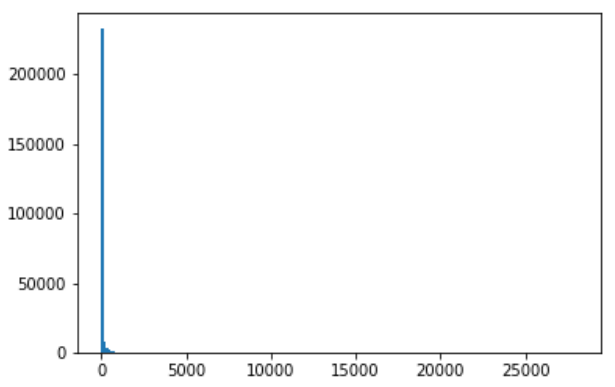

(a) All Posts

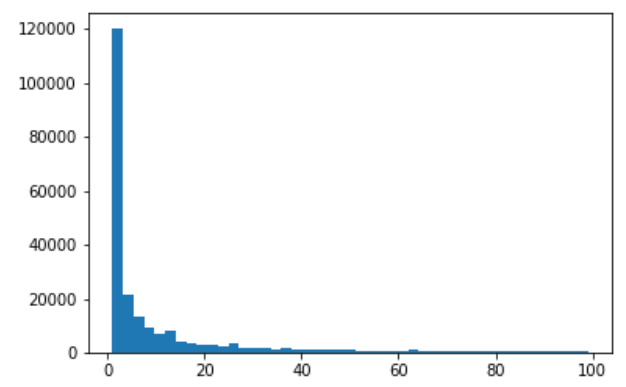

(b) Filtered to 100

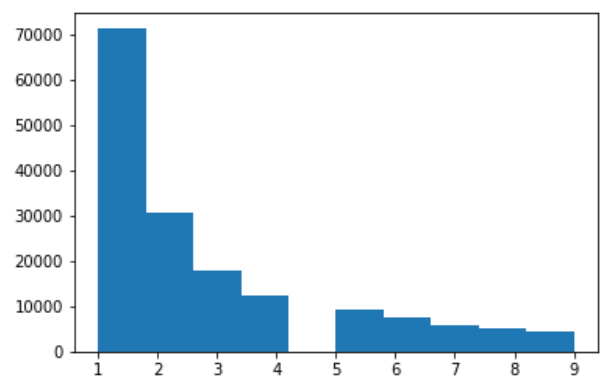

(c) Filtered to 10

Figure 11. Histogram showing the Posts per user. This shows a histogram for all users (a), and zooms in towards the from of the exponential distribution by filtering authors with less than 100 posts (b) and 10 posts (c) 


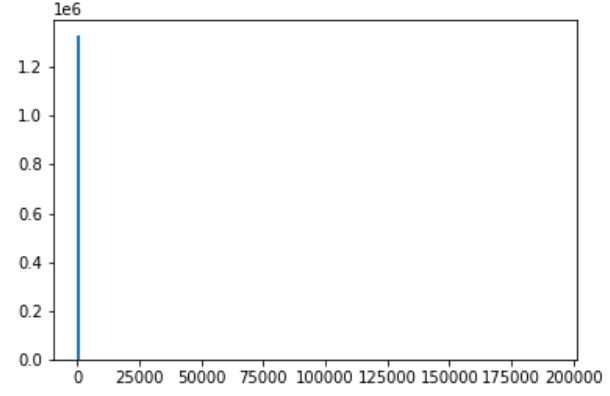

(a) All Posts

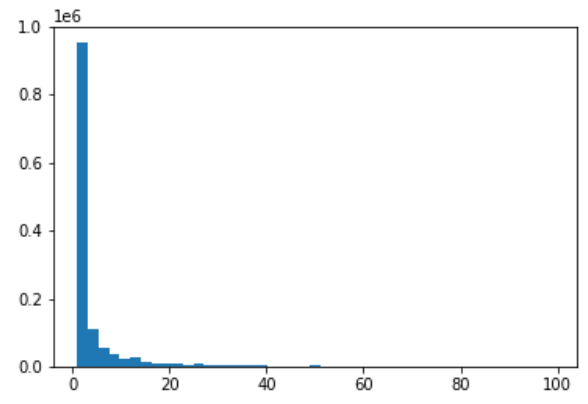

(b) Filtered to 100

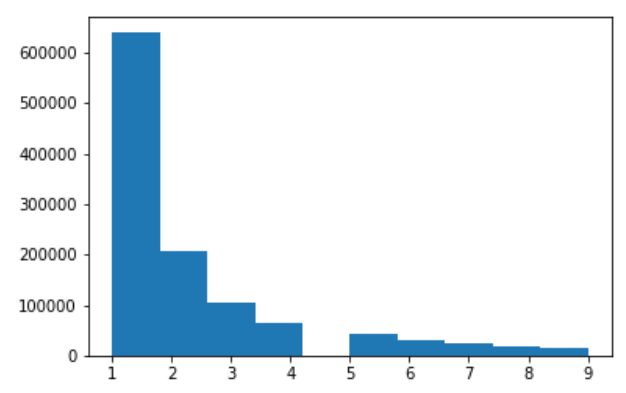

(c) Filtered to 10

Figure 12. Histogram showing the Posts per user. This shows a histogram for all users (a), and zooms in towards the from of the exponential distribution by filtering authors with less than 100 posts (b) and 10 posts (c) 


\section{CHAPTER III}

\section{LINGUISTIC TASKS}

Algorithm development for each psychological and behavioral biometric needs to be

done before any experimentation on translations can occur. A literature review of techniques and methods used by other authors is conducted for each of the tasks, though Personality Trait Estimation has been explored far less than the others. For both Affect and Personality Trait estimation, we use specific literature that appeared to be most promising and further explored those systems in an attempt to improve the English language system. Ablation testing helped determine some of the potential machine learning hyperparameters and features used in the systems.

Several existing research studies were used for determining the Authorship attribution algorithms used in this dissertation. This section discussed the algorithms used, and the performance of those algorithms against the CASIS dataset.

For both affect analysis and authorship attribution, the performance of the models is tested within the native foreign language. Due to the limitations of the Personality estimation task, exploration into a foreign language using this dataset is impossible. In future research efforts, conducting personality trait estimation in a native language should be explored - especially a non-Latin based language to determine the efficacy of the algorithm in various rooted languages. 


\section{Affect Analysis}

As mentioned in Chapter II. Affect and Sentiment Analysis are tangential tasks that seek to measure specific states of the person being analyzed. The distinguishing factors between the two tasks are the intended measurements. In Sentiment Analysis, the user seeks to identify the target's valence (whether they feel positively or negatively) towards a topic. Affect analysis, on the other hand, attempts to measure the intensities of the emotions expressed by an author as a gauge of their overall emotional state, regardless of the topic being discussed [93]. This gauge of emotional state can lead to a more significant understanding of the person at any given time and help understand how their emotional states might lead to further, potentially malicious actions.

Sentiment Analysis has been more widely researched. The most common method that has been used in Sentiment Analysis involved the use of fixed lexicons. Grefenstette et al. [94], a manually generated lexicon created by Subasic et al. [97] was used to identify words and their associations to emotions. Words like 'gleeful' could be associated with multiple emotions. In their example, they associate the word to both 'Happiness' and 'Excitement.' The words are then weighted according to which emotion the word is most likely to express. The same study also looked at emotive patterns to help in the creation of a seed list. Phrases such as, "looking extremely ...", would be added to the list. The phrases would then be looked for within internet sources to identify the following word used as part of the lexicon. Ma et al. [95] utilized the WordNet-Affect Database [151, 152] to help them study and understand emotions within conversations. Studies from Chuang et al. [98 manually created emotional descriptors based on a Chinese lexicon of 65,620 words. These were then manually mapped to their specific emotions.

While lexicons provide an easy way to map text to emotions, it does not afford the ability for semantic change to be captured. Semantic change is a form of language 
change to the evolution of the meaning of words. According to Leonard Bloomfield [153], word meanings can shift over time. This shift can occur for many reasons: when meanings are narrowed or widened, changed by another meaning that is similar to the original meaning, by an elevation or lowering of the status of the original meaning, when the word for part of something begins to be used to reference the whole of the system, when nouns are replaced by something close to the meaning, or when exaggerations and understatements change the meaning of the words. While the most common words rarely change, the meanings of various English words have changed over time. This form of semantic change is common in modern colloquialisms and slang [154]. This limitation in capturing semantic change within lexicons means that lexicons should be manually updated with modern colloquialisms every few years to capture emotion within the new contexts.

Other studies in the area of Sentiment and Emotion Analysis have utilized Word ngrams and parts-of-speech n-grams. Mishne initially focused on the use of frequency counts for word n-grams and parts-of-speech n-grams within a Blog corpus [100]. Later experimentation from Mishne looked at additional Natural Language Processing features, including frequency of special characters and post lengths. While this process would have to be updated for consideration of semantic changes as well, the use of these Natural Language processing features can assist in creating a more automated approach for continually updating the model and automatically capturing semantic change.

Abbasi and Chen [46, 102, 93] utilized many of these features in building their models, utilizing both lexicons, word n-grams, part-of-speech n-grams, character ngrams, and counts of hapax and dis legomina. With these features, the Support Vector Regression model determined the best features for each of the identified emotions. This SVR model was then run against a dataset created from forum posts to identify potential terrorist activity. It is unclear whether their model utilized translations of 
the Arabic text or worked within the original Arabic script. In either case, the model proposed by Abbasi and Chen provides the basis for the development of the affect system used in further experimentation in this dissertation.

\section{Algorithm Design}

The algorithm proposed by Abbasi et al. [46, 102, 93] appeared to be one of the most promising algorithms for further experimentation. Their proposed algorithm was adapted for the SemEval-2018 dataset discussed in Chapter III. The use of Correlation Ensembles was not possible with the SemEval dataset, so the Correlation Ensemble aspect is not used in the analysis from the analysis. Instead, an Ensemble of Support Vector Regression (SVR) models are used to calculate each of the emotions within the SemEval-2018 dataset.

Abbasi et al. [46, 102, 93] also discuss the features that are used in their algorithm. Abbasi et al. [102] use various types of n-grams, including Character, Word, and Parts-of-Speech n-grams for their analysis, and only up to trigrams (3-grams). In their studies, they also include "hapax legomina" and "dis legomina" collocations. Removal of these collocation occurrences removes words that only happen once or twice, respectively, in the entirety of the dataset, removing sparsely occurring words allows the n-grams to generalize more over the entire dataset.

Before fitting the data to the SVR model for each emotion, numerous preprocessing steps are conducted for the word n-grams and parts-of-speech n-grams. Each tweet is tokenized with a Twitter-aware tokenizer. All tokens are converted to lowercase, contractions are expanded, and all slang words are replaced. All contractions and slang replacements are retokenized to avoid cases where the expansion or replacement introduces additional word tokens into the text. Any tokens consisting of punctuation or that are empty are removed from the token list. Once all of the tokens are obtained, stemming and lemmatization of the words occurs in order to 
group inflected forms of words (e.g. "Better" and "Good" share the same lemma, "Running" is stemmed so that it shares a lemma with "run"). Once preprocessing is completed, the tokens can be treated as words. These words are used to calculate the parts-of-speech for use in training the system.

Support Vector Regression uses many of the same principles as Support Vector Machines, with minimal differences. Since the output of SVR models are real numbers, making prediction more difficult since this introduces an infinite possibility space. To resolve this, SVR introduces a margin of tolerance $(\epsilon)$. This $\epsilon$ is used to help the algorithm only consider those points within the set margin, ignoring those data points with a more significant error rate. The margin of tolerance allows the algorithm to create a better fitting model using whichever kernel. SVR models are created for each of the emotions within the tested datasets.

A correlation ensemble algorithm is used when emotions are correlated. Correlations of emotions means that a single tweet or message contains a list of all emotions calculated in the model. In both the SemEval-2007 and Arabic datasets that are tested, this means that every tweet or news headline contains values for the entire list of Ekman emotions. The algorithm for correlation ensembles is outlined in [102] and rewritten in Algorithm 1 .

\section{English Performance}

The Kernel, Regularization Parameters, and $\epsilon$ for each of the SVR models is optimized using a Grid Search method, an exhaustive search over the hyperparameter space. These parameters include a Linear kernel, Polynomial kernel, and Radial Basis Function kernel in the kernel space; $[0.1,1,1.5,2]$ in the regularization parameter, 'C' space; and $[0.05,0.1,0.15,0.2,0.3,0.4,0.5]$ in the $\epsilon$ space. These features are ranked using the Root Mean Squared Error obtained from the regression model.

This grid search is also conducted on the powerset of features tested: character n- 


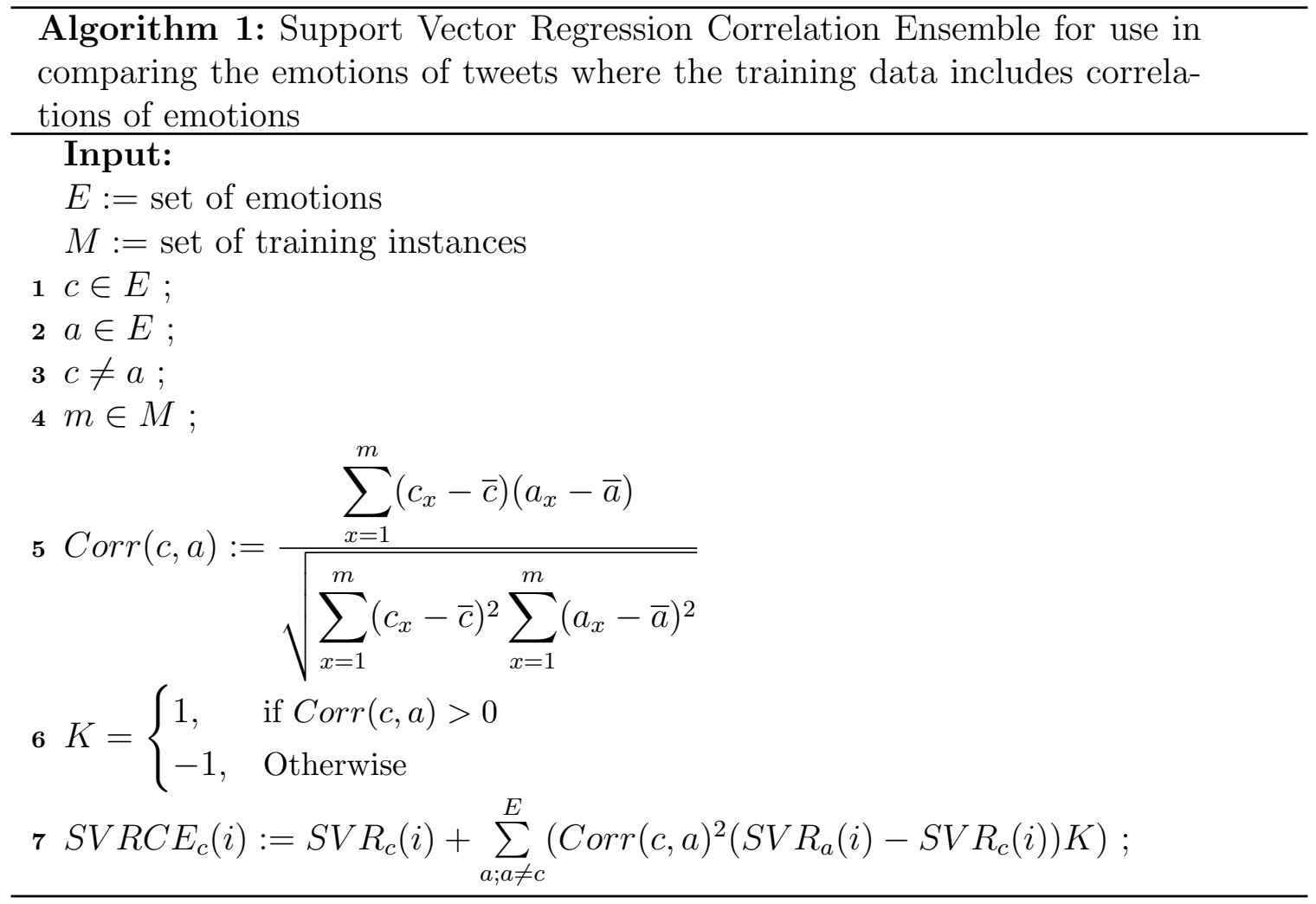

grams, word n-grams, parts-of-speech n-grams, and unprocessed word n-grams. Due to Python's limitations, the hyperparameters are selected programmatically, but the feature selection is made manually. For each emotion and feature, a grid search optimization for the hyperparameters is run. The RMSE values are then calculated using a 10-fold cross-validation on the same dataset using a $10 \%$ split for the test set. Table 8 shows the results of the grid search optimization. We see that both Anger and Joy provide the lowest RMSE when both character- and word- n-grams are selected; fear is best optimized when using character-, word-, and the vanilla n-grams; and sadness is best optimized when parts-of-speech-, character-, and word- n-grams are used. Table 9 shows the results of the Grid Search optimization of the SemEval-2018 dataset for each hyperparameter and feature optimized.

With the optimized hyperparameters and feature sets, various metrics are calculated again using a 10-fold cross-validation of the algorithm on the dataset with a $10 \%$ split for the test set. The Error metrics selected include $R^{2}$, when the kernel is 
linear; Mean Absolute Error (MAE); Mean Squared Error (MSE); and Root Mean Squared Error (RMSE). $R^{2}$ is not reported for non-linear kernels because the method of calculation can result in high values, but, due to the non-linear function used for the regression model, the value could be meaningless in determining the goodness of fit when the model has wide confidence intervals. Table 10 shows the calculated metrics for each of the optimized emotion models.

Based on the metrics in Table 10, we see that the $R^{2}$ scores are consistent with many psychological studies at slightly 0.5 . Human behaviors are harder to predict, and often result in a lower coefficient of determination. The scores around 0.5 for Anger, Fear, and Joy are consistent with many human behavior studies and show a goodness-of-fit for the linear models that are good with estimations of human behaviors. The $R^{2}$ for the Sadness trait is extremely good for predicting sadness within the system as it is nearly a value of one. This high metric may be caused by an over-fitting of the data or indicate how sadness is expressed through twitter. This expression may be shared among a large population of users. Further research into the linguistic and psychological understanding of this identified phenomenon is necessary to ascertain this model's generalizability to datasets outside of the acquired datasets.

Both the Mean Absolute Error (MAE), Mean Squared Errors (MSE), and Root Mean Square Errors (RMSE) are methods that summarize the performance of the system without regard to whether the algorithm over or underpredicted the outcome. The MAE shows that the estimates of the traits falls within that range most of the time. The scales for the emotions are normalized from $[0,1]$ inclusive. An MAE for Anger of 0.1034 means most of the errors observed in the system will be off by plus or minus .10. RMSE provides the same scale that the system operates in - in this case, the RMSE should be a value between $[0,1]$. RMSE weighs the higher magnitude error rates in the system more than MAE, providing a sense of how large the system's error 


\begin{tabular}{|l|r|r|r|r|}
\hline & Anger & Fear & Joy & Sadness \\
\hline \{'word'\} & 0.1364 & 0.1845 & 0.2011 & 0.1593 \\
\hline \{'pos'\} & 0.1739 & 0.1939 & 0.2045 & 0.1958 \\
\hline \{'vanilla'\} & 0.1680 & 0.1859 & 0.1971 & 0.1618 \\
\hline \{'char'\} & 0.1352 & 0.1426 & 0.1493 & 0.1927 \\
\hline \{'pos', 'word'\} & 0.1370 & 0.1880 & 0.1572 & 0.1790 \\
\hline \{'word', 'vanilla'\} & 0.1347 & 0.1777 & 0.1522 & 0.1599 \\
\hline \{'pos', 'vanilla'\} & 0.1395 & 0.1887 & 0.1640 & 0.1683 \\
\hline \{'char', 'word'\} & 0.1284 & 0.1379 & 0.1461 & 0.1900 \\
\hline \{'pos', 'char'\} & 0.1373 & 0.1502 & 0.1561 & 0.0455 \\
\hline \{'char', 'vanilla'\} & 0.1310 & 0.1389 & 0.1487 & 0.1904 \\
\hline \{'pos', 'word', 'vanilla'\} & 0.1349 & 0.1871 & 0.1546 & 0.1641 \\
\hline \{'pos', 'char', 'word'\} & 0.1311 & 0.1402 & 0.1489 & 0.0452 \\
\hline \{'char', 'word', 'vanilla'\} & 0.1302 & 0.1370 & 0.1463 & 0.1883 \\
\hline \{'pos', 'char', 'vanilla'\} & 0.1329 & 0.1412 & 0.1515 & 0.0455 \\
\hline \{'pos', 'char', 'word', 'vanilla'\} & 0.1312 & 0.1393 & 0.1484 & 0.0450 \\
\hline
\end{tabular}

Table 8. This table shows the RMSE values for each of the emotions and the features within the SemEval-2018 dataset. Blue highlighted cells in each column represent the feature combinations that provided the lowest RMSE rate. Other features in the Gridsearch - Regularization parameter, $\epsilon$, and kernel are not shown and were chosen programmatically.

\begin{tabular}{|l|l|r|r|l|}
\hline & Features & $\mathrm{C}$ & $\epsilon$ & Kernel \\
\hline Anger & $\{$ 'char', 'word'\} & 0.10 & 0.05 & Linear \\
\hline Fear & $\{$ 'char', 'word', 'vanilla' $\}$ & 0.10 & 0.05 & Linear \\
\hline Joy & $\{$ 'char', 'word'\} & 0.10 & 0.05 & Linear \\
\hline Sadness & \{'pos', 'char', 'word'\} & 10.00 & 0.05 & Linear \\
\hline
\end{tabular}

Table 9. The selected features from a Grid Search Optimization against the SemEval-2018 data. These parameters provide the lowest error rates against twitter data.

\begin{tabular}{|l|r|r|r|r|}
\hline & $R^{2}$ & MAE & MSE & RMSE \\
\hline Anger & 0.4594 & 0.1034 & 0.0167 & 0.1284 \\
\hline Fear & 0.4965 & 0.1090 & 0.0188 & 0.1370 \\
\hline Joy & 0.4944 & 0.1163 & 0.0214 & 0.1461 \\
\hline Sadness & 0.9464 & 0.0432 & 0.002 & 0.0452 \\
\hline
\end{tabular}

Table 10. Performance metrics calculated for each of the optimized English emotion models. Metrics include: $R^{2}$ for linear models (N/A is shown when a non-linear model is selected), Mean Absolute Error (MAE), Mean Squared Error (MSE), and Root Mean Squared Error (RMSE). 
rates are. We see that the RMSE of all of the system increases over the MAE, meaning that while we can expect the errors to average around the RMSE, we can expect that we will have some significantly larger errors in the system from some of the input text. However, these metrics show that the system used for our experimentation is competitive with other Algorithms used against the SemEval-2018 dataset [119] and Abbasi et al.'s algorithm performance against short Fifty-word short story text [102].

\section{Arabic Performance}

Since the system built for this experiment is competitive with other emotion estimation systems, a similar methodology was used to determine the effectiveness of these algorithms against the Arabic Emotion dataset. Each hyperparameter and powerset of features were again optimized against the dataset using a grid search method. These parameters again include a Linear kernel, Polynomial kernel, and Radial Basis

Function kernel in the kernel space; $[0.1,1,2,5,10]$ in the regularization parameter, $C$ space; and $[0.05,0.1,0.15,0.2,0.3,0.4,0.5]$ in the $\epsilon$ space. This grid search was ranked using the Root Mean Squared Error obtained from the regression model.

There are far fewer tools for experimentation in the Arabic space. Stanford University's CoreNLP [155] is one of the few research tools available for Natural Language Processing in Arabic. CoreNLP implements lemmatization in the language, Parts-ofspeech taggers, Named-Entity Recognition, and other tools that Stanford's Natural Language Processing Group has designed. Columbia University has also developed MADAMIRA [156], which improved Morphological Arabic analysis. However, this tool is more complicated to set up and requires access to the Linguistic Data Consortium's Standard Arabic Morphological Analyzer (SAMA) [157]. The University of Maryland [158] also created an improved tool for lemmatization, but this tool requires MADAMIRA as a dependency. For simplicity, analysis in this section is completed using Stanford's CoreNLP. Future research should analyze potential improvements 
using the University of Maryland's Arabic Toolkit, combined with Columbia University's MADAMIRA.

Analysis of the Arabic data used character n-grams, word n-grams, and partsof-speech n-grams. The Stanford CoreNLP tool was used to tokenize words and determine the parts-of-speech in the language. Since other tools used in the English preprocessing were not designed for analysis of foreign languages, we rely solely on the tokenization conducted by CoreNLP. Due to limitations in Python, the hyperparameters are selected programmatically, but the feature selection is made manually. For each emotion and feature, a grid search optimization for the hyperparameters is run. The RMSE values are then calculated using a 10-fold cross-validation on the same dataset using a $10 \%$ split for the test set. Table 11 shows the results of the grid search optimization. We see that both Anger and Joy provide the lowest RMSE when both character- and word- n-grams are selected; fear is best optimized when using character-, word-, and the vanilla n-grams; and sadness is best optimized when parts-of-speech-, character-, and word- n-grams are used. Table 12 shows the results of Grid Search optimization against the SemEval-2018 dataset for each hyperparameter and features that were optimized. Performance metrics were again calculated using a 10-fold cross-validation with a 10\% holdout and are shown in Table 13 .

Based on the metrics in Table 13, we do see a degradation in performance over the English language data. Except for Joy, the $R^{2}$ values of Anger, Disgust and Fear now sit in the lower- to mid- 0.3s. These scores indicate that the methods used here are not quite to the same level of the English language preprocessing or feature sets. Future work should explore other methods for preprocessing and feature extraction. The University of Maryland toolkit appears to handle various preprocessing steps similar to the NLP tools in English. This toolkit should provide improvements to the algorithms.

The MAE shows some degradation in emotions as well. The algorithm simply has 


\begin{tabular}{|l|r|r|r|r|r|r|}
\hline & Anger & Disgust & Fear & Joy & Sadness & Surprise \\
\hline \{'word'\} & 0.2742 & 0.1763 & 0.1258 & 0.3274 & 0.2470 & 0.1837 \\
\hline \{'pos'\} & 0.2656 & 0.1996 & 0.1448 & 0.2957 & 0.2420 & 0.1839 \\
\hline \{'char'\} & 0.2760 & 0.1995 & 0.1455 & 0.3418 & 0.2483 & 0.1838 \\
\hline \{'pos', 'word'\} & 0.2246 & 0.1770 & 0.1279 & 0.3327 & 0.2477 & 0.1838 \\
\hline \{'char', 'word'\} & 0.2757 & 0.1623 & 0.1191 & 0.3398 & 0.2482 & 0.1838 \\
\hline \{'pos', 'char'\} & 0.2121 & 0.1643 & 0.1233 & 0.2219 & 0.2484 & 0.1838 \\
\hline \{'pos', 'char', 'word'\} & 0.2112 & 0.1637 & 0.1209 & 0.3397 & 0.2482 & 0.1838 \\
\hline
\end{tabular}

Table 11. This table shows the RMSE values for each of the emotions and the features within the Arabic dataset. Blue highlighted cells in each column represent the feature combinations that provided the lowest RMSE rate. Other features in the Gridsearch - Regularization parameter, $\epsilon$, and kernel are not shown and were chosen programmatically.

\begin{tabular}{|l|l|r|r|l|}
\hline & Features & $\mathrm{C}$ & $\epsilon$ & Kernel \\
\hline Anger & \{'pos', 'char', 'word'\} & 0.10 & 0.10 & Linear \\
\hline Disgust & $\{$ 'char', 'word'\} & 0.10 & 0.05 & Linear \\
\hline Fear & $\{$ 'char', 'word'\} & 0.10 & 0.10 & Linear \\
\hline Joy & \{'pos', 'char'\} & 0.10 & 0.10 & Linear \\
\hline Sadness & $\{$ 'pos'\} & 0.10 & 0.10 & RBF \\
\hline Surprise & $\{$ 'word'\} & 1.00 & 0.05 & RBF \\
\hline
\end{tabular}

Table 12. The selected features from a Grid Search Optimization against the Arabic data. These parameters provide the lowest error rates against arabic twitter data.

\begin{tabular}{|l|r|r|r|r|}
\hline & $R^{2}$ & MAE & MSE & RMSE \\
\hline Anger & 0.3573 & 0.1448 & 0.0447 & 0.2112 \\
\hline Disgust & 0.3339 & 0.0831 & 0.0264 & 0.1623 \\
\hline Fear & 0.3193 & 0.0539 & 0.0143 & 0.1191 \\
\hline Joy & 0.4704 & 0.1624 & 0.0492 & 0.2219 \\
\hline Sadness & N/A & 0.1654 & 0.0586 & 0.2420 \\
\hline Surprise & N/A & 0.0954 & 0.0339 & 0.1837 \\
\hline
\end{tabular}

Table 13. Performance metrics calculated for each of the optimized Arabic emotion models. Metrics include: $R^{2}$ for linear models (N/A is shown when a non-linear model is selected), Mean Absolute Error (MAE), Mean Squared Error (MSE), and Root Mean Squared Error (RMSE). 
a higher magnitude error rates over the English algorithms; however, the significant increase in the RMSE shows that the errors that are being obtained by the algorithm tend to be heavily weighted toward higher magnitude errors. In the English language analysis, we see that the MAE and RMSE values were relatively close, indicating that there were few higher magnitude errors to weight the RMSE higher. The larger RMSE on the Arabic dataset indicates that there are likely some much higher magnitude errors occurring in the data.

While this algorithm is perhaps not quite at the same level as the same algorithm on English language text, further experiments in this dissertation show that the use of translations significantly impacts results. This finding indicates that further exploration of Emotion analysis in the native language is necessary to improve foreign languages' performance.

\section{Personality Trait Estimation}

Automatic Personality Recognition, or the inference of the self-reported personality of an individual from behavioral evidence, has slowly been studied, but with the increased usage of social media, psycholinguistic analysis of the text has become a common practice. Tausczik and Pennebaker [159] state it most concisely: "Language is the most common and reliable way for people to translate their internal thoughts and emotions into a form that others can understand. [...] [Words and language] are the medium by which cognitive, personality, clinical, and social psychologists attempt to understand human beings."

Youyou's 2015 study [160] found that Computer judgments of personality traits exceed human judgments on the same tasks. Computer judgments correlated (Pearson's $r$ of 0.56$)$ more strongly with participants self-ratings over human judgments (Pearson's $r$ of 0.49). Their study also found that computer models were better at predicting life outcomes and other behaviorally related traits than human judgments. 
The use of computer models to estimate personality traits provides not only superior judgments, according to Youyou, but can also help to assist psychologists in analyzing more copious amounts of data, as discussed in Chapter I]

The use of text as a medium for estimating personality traits has been studied since 2005. In 2005, Argamon et al. [161] used written essays and a constructed lexicon using attribute-value taxonomies to estimate both Extraversion and Neuroticism, achieving a 58\% accuracy in their Support Vector Machine classifier. Mairesse et al. [162] also utilized written essays and the Linguistic Inquiry and Word Count (LIWC) [159] and the Medical Research Council (MRC) Psycholinguistics Database [163] to estimate all five traits within the Five-Factor Model, achieving Mid-50\% accuracy on Extraversion, Agreeableness, Conscientiousness, and Neuroticism, and 62\% accuracy on Openness. While both of these methods relied on the use of fixed Lexicons, Oberlander and Nowson [164, 165] used blog posts and word n-grams to achieve slightly higher accuracy over the Lexicon methods, achieving mid-50\% accuracy for Extraversion and Neuroticism, while achieving 61\% accuracy on Agreeableness and $65 \%$ accuracy for Conscientiousness.

Social media platforms have been more commonly studied since at least 2011, as social media provides unique platforms for the study of social sciences. Golbeck, Robles, and Turner [166] used a small sample of Facebook Profiles to estimate the Big 5 personality traits. The features include: profile images, whether their profile was set to block anonymous users; activities of the users; favorite books; the number of friends; Egocentric Network Density; and various linguistic features. With these features, the authors were able to achieve Mean Absolute Errors (MAE) of 0.10 for Openness; 0.10 for Conscientiousness; 0.14 for Extraversion; 0.11 for Agreeableness; and 0.13 for Neuroticism. MAE often underweights higher magnitude errors that might be observed in the system, providing inaccurate results for what to expect. However, the use of other non-linguistic features might also play a role in the perfor- 
mance of their estimation of the Big 5 traits.

Golbeck et al. [167] and Quercia et al. [168] also looked at the use of Twitter as a mechanism for estimating personality traits in 2011. Golbeck et al. used a set of 2,000 tweets per their 279 authors. Their features included profile information provided by Twitter and the use of LIWC and MRC lexicons for estimating personality traits. Their system achieved MAEs of 0.12 for Openness; 0.14 for Conscientiousness; 0.16 for Extraversion; 0.13 for Agreeableness; and 0.18 for Neuroticism. Quercia et al. estimated personality traits just based on 335 twitter profiles, using features such as the number of followers, number of people following, and listed counts. No linguistic analysis was conducted during this study since the authors were attempting to estimate the personality of private twitter users. They achieved a Root-Mean-Squared-Error (RMSE) of 0.69 for Openness; 0.76 for Conscientiousness; 0.88 for Extraversion; 0.79 for Agreeableness; and 0.85 for Neuroticism. The use of RMSE provides better insight into the overall performance of the human prediction task since it emphasizes higher magnitude errors.

A Computational Personality Recognition Workshop was held at a 2013 AAAI conference [169]. Several interesting findings came out of this workshop: feature selection was most effective when a ranking algorithm was used over the initial feature space; use solely on word n-grams was not very useful; use of lexical resources (lexicons) in general seemed to be the most useful; ensemble methods seemed to perform the best in the prediction of personality traits; that cross-domain learning (e.g., using essays to train against Facebook status) is possible. These findings played an essential role in selecting features in the algorithm used in experimentation in this dissertation.

The algorithm used in this dissertation is meant to estimate personality solely based on a linguistic analysis. While it has been shown that other profile features may provide additional insight into personality, one cannot assume that the profile in- 
formation is always available. This assumption that a profile would be provided is especially wrong on various dark web platforms where users commonly seek anonymity for their activities. However, understanding their personality is still an important aspect.

A proprietary algorithm is used for further experimentation around the estimation of personality traits. This section will discuss details about the algorithm's creation, and provide some insight into the performance of the system when tested against the dataset described in Chapter II. RMSE was the primary measure for the task to ensure that we consider the overall performance and emphasize higher magnitude errors.

\section{Algorithm Design}

The algorithm used in our experimentation is based on the work by Farnadi et al. [170], Park et al. [171], and Schwartz et al. [172]. Schwartz et al. [172] used Latent Dirichlet Allocation [173] to generate open-vocabulary topics for all of the documents compared against, allowing for correlations between the topics and the personality traits. The studies of [171] and [172] looked at other features, such as LIWC, but there seemed to be minimal impact overall when LIWC was used in conjunction with topic modeling. The proprietary algorithm used in our experimentation avoids the use of LIWC due to the current costs of the most recent version.

Farnadi et al. [170] provide state of the art RMSE for a personality estimation system. The ensemble created in their system uses various feature sets against each of the Big 5 personality Traits. Their system achieves an RMSE of 0.651 for Openness using Facebook activity, demographic information, LIWC, MRC, SentiStrength Lexicons, and the Structure Programming for Linguistic Cue Extraction (SPLICE) features. A RMSE of 0.717 for Conscientiousness using the same features as Openness. A RMSE of 0.784 for Extraversion using only Facebook Activity and demographic 


\begin{tabular}{|l|r|}
\hline Algorithm & RMSE \\
\hline Linear Regression & 0.82 \\
\hline Support Vector Regression (RBF Kernel) & 0.82 \\
\hline Decision Tree & 0.78 \\
\hline K Nearest Neighbors & 0.76 \\
\hline Radial Basis Neural Network & 0.75 \\
\hline
\end{tabular}

Table 14. Root Mean Squared Error for each of the tested algorithms for selection. Features were the 2000 topics identified in earlier testing.

information. A RMSE of 0.692 for Agreeableness using the same features as Openness. Lastly, a RMSE of 0.768 for Neuroticism using only Facebook Activity data and demographic information. We compare the developed algorithm to Farnadi et al.'s system to compare the algorithm against state of the art.

Various methods that have been found in the literature were tested to determine the optimum. Ablation testing was conducted to determine the optimal number of topics and which algorithms performed the best. Table 14 shows the RMSE values of various algorithms against a 2,000 topic model to determine the most optimal algorithm to use in experimentation. We see through observation that the use of Radial Basis Neural Networks provided the best mean RMSE for all Regression models. This model was chosen to optimize further.

While LDA was used to generate a 2,000 topic feature set, optimization was conducted on the Radial Basis Neural Network to determine the number of topics that provide the optimal number of topics for the training set. Table 15 shows the RMSE values for topics from 200 to 5,000 topics. We see that we start to achieve optimal results between 1,000 and 2,000 topics. While further optimization could produce slightly improved results, 2,000 topics were selected to avoid any potential over-fitting of the model and simplify the selection of the features. These topics were generated using an optimized version of collapsed Gibbs Sampling within the LDA model. While Variational Bayes is a computationally faster approach, the Variational Bayes model often results in a less accurate model. 


\begin{tabular}{|l|r|}
\hline \# Topics & RMSE \\
\hline 200 & 0.89 \\
\hline 500 & 0.95 \\
\hline 750 & 1.1 \\
\hline 1000 & 0.78 \\
\hline 2000 & 0.75 \\
\hline 5000 & 0.84 \\
\hline
\end{tabular}

Table 15. Root Mean Squared Error for set numbers of topics used to determine the number of topics to use for personality estimation. RMSE given is the average RMSE for all five personality traits.

By running LDA with 2,000 topics against the dataset described in Chapter II, we see that we can achieve a RMSE error comparable to the results in Farnadi et al. Achieving a mean RMSE between the five personality traits of 0.75 , utilizing only the linguistic data in the dataset. Further ablation tests of other Natural Language Processing features occurred, and the following features were found to provide additional benefits to the algorithm:

- Age

- Gender

- Total number of words

- Count of Hapax and Dis Legomina

- Yule's K Measure

- M1 and M2 values computed during the calculation of Yule's K measure

- Sichel's Measure

- Brunet's Measure

- Honore's Measure

- Count of Negative Sentiment Words 


\begin{tabular}{|l|r|}
\hline Trait & RMSE \\
\hline Openness & 0.469 \\
\hline Conscientiousness & 0.628 \\
\hline Extraversion & 0.721 \\
\hline Agreeableness & 0.557 \\
\hline Neuroticism & 0.736 \\
\hline Mean All 5 & .630 \\
\hline
\end{tabular}

Table 16. Root Mean Squared Error for each personality trait with 2,000 topics and the additional NLP features.

With these 2,011 different features, Singular Value Decomposition is used to reduce the overall feature set to 100 features. These decomposed features become the inputs to the Radial Basis Network, with the outputs of the Network expected as the estimate of the personality trait being tested. Each personality trait is a separate model, creating an ensemble of five algorithms for estimating each of the personality traits. Table 16 shows each of the final RMSE scores for each of the five traits using the described model.

These RMSE values show significant improvements over Farnadi et al., indicating that further optimization of their algorithms could increase scores. While this is interesting, the algorithm described here provides a platform for further testing of the system against translations in Chapter IV] However, further sensitivity analysis is conducted to determine the optimal number. It provides additional insight into the requirements for the dataset to ensure that we can limit the users to some minimal criteria that will provide the more considerable insight into the effect of translations on the system.

Using the optimized algorithm, we test the documents of varying word lengths against each of the personality traits to determine the impact to the RMSE of smaller numbers of words. Figure 13 shows the word sensitivity at different document word lengths. We see that using fewer than 500 words results in a significant increase in the RMSE of each personality trait. We see that each of the lines appears to begin 


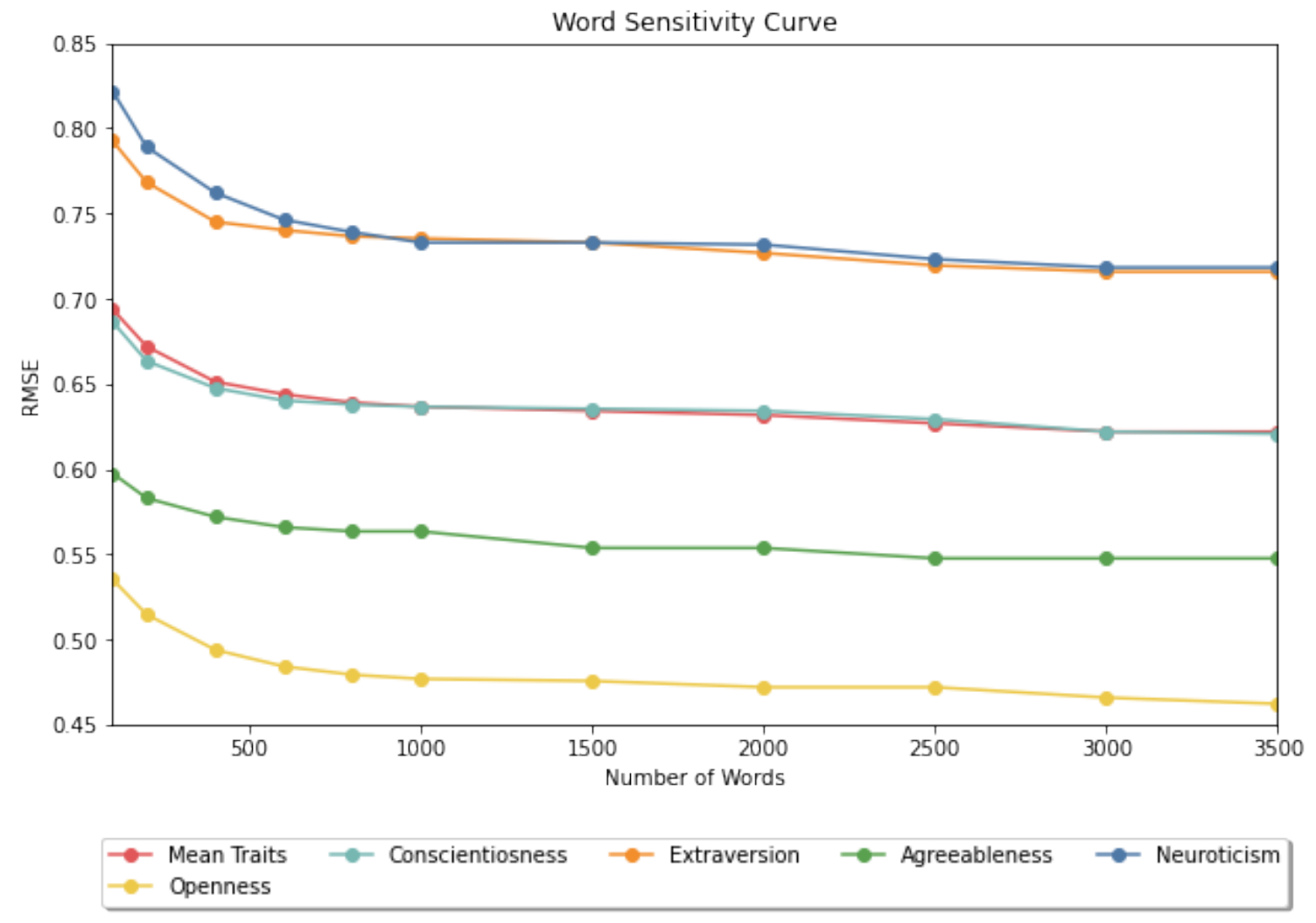

Figure 13. Word Sensitivity for the RMSE of the personality trait estimation algorithm.

to level off at around 1,500 words with only minor gains in RMSE as more words are added. For this reason, a minimum threshold of 1,500 words per document is required to test the system for optimal performance properly. This document word length is used for further experimentation.

\section{Authorship Attribution}

Authorship Attribution and Verification is a behavioral biometric intended to identify or verify the user based on a stylometric analysis of the author's text. In 2017, Sundararajan et al. [174] and Neal et al. [89] conducted a survey analysis of fourteen prominent authorship attribution algorithms based on the work of Potthast et al. [175]. The University of Florida study was intended to determine the applicability of the fourteen algorithms to large datasets, compare sentence lengths, and outline 
the challenges of authorship attribution at a massive scale. These studies found six algorithms that perform well across large datasets.

\section{Algorithms}

This section of the dissertation replicates the analysis conducted in [174] to estimate the performance metrics of 4 prominent algorithms in the study. Due to computational resources available in this study, the dissertation then chooses a single algorithm to measure various metrics in English and conduct further studies to determine the effect of translations on the algorithm. A more extensive study based on this work is currently ongoing between this author and the University of Florida. It will measure the effects of translations on all four of the prominent algorithms.

Four algorithms were selected from the study. These include the Keselj [176], Koppel [177], Stamatatos [178], and Teahan [179] algorithms. Each of these algorithms uses different techniques for determining authorship.

The Keselj algorithm [176] uses pairs of character n-grams and the corresponding frequencies of those n-grams. The algorithm was designed to be language-agnostic and was tested by Keselj on English, Greek, and Chinese datasets. The decisions made by the authors were intentionally made to make the algorithm as languageindependent as possible. The work was based on an algorithm proposed in 1976 by Bennett [180], which utilized a similarity metric requiring frequencies of bigram occurrences in "Standard English." Keselj utilized a metric that removes this languagedependent parameter and utilizing measured bigram frequencies. After measuring the frequency of bigram occurrences, two profiles are compared using Algorithm 2 proposed by Keselj. Once the dissimilarity measure is compared, the author that minimizes the dissimilarity measure is matched, and the anonymous text is aligned with that author's profile.

The Stamatatos algorithm [178] is very similar to the Keselj algorithm, using 

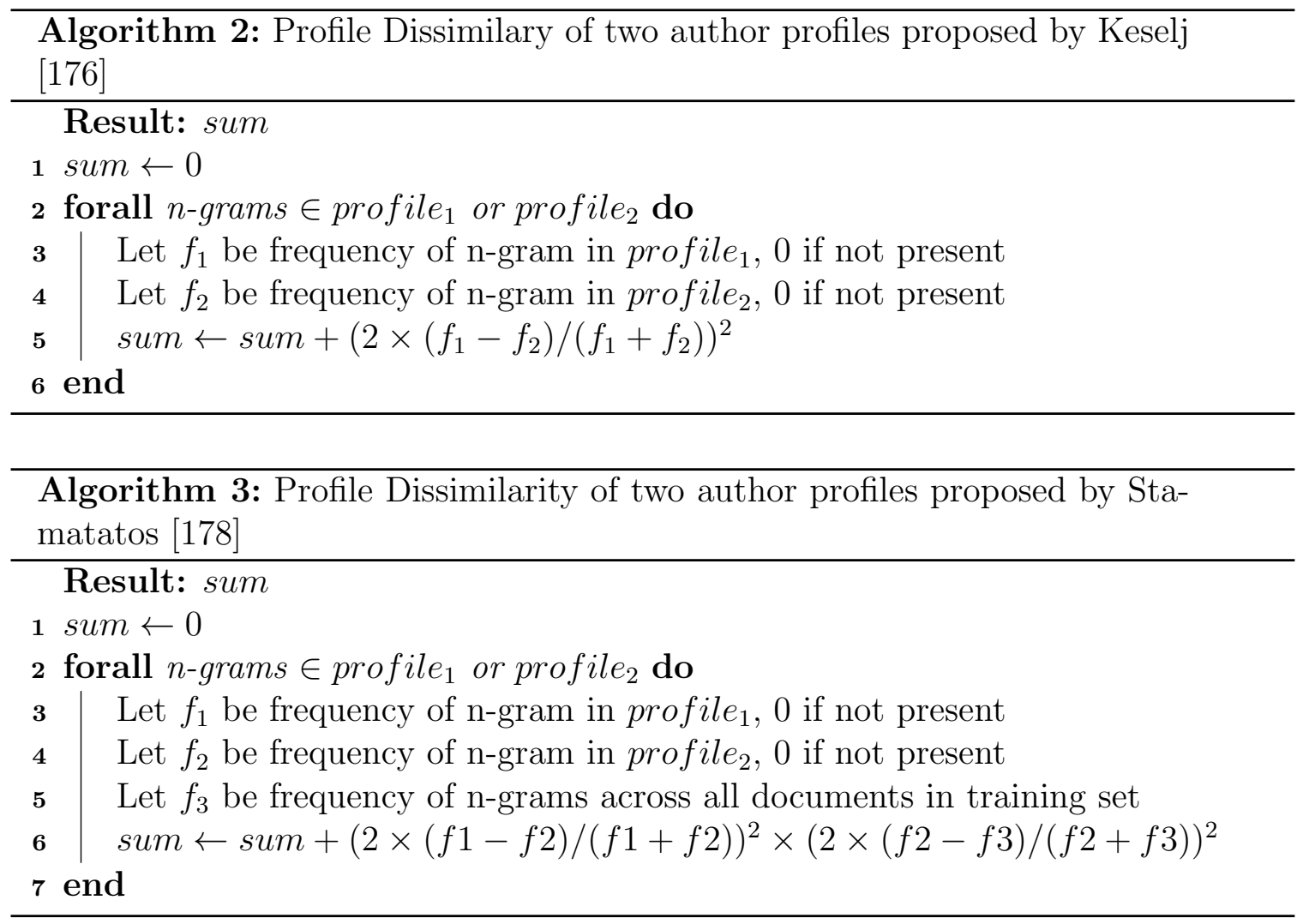

pairs of n-grams and the corresponding frequencies of those n-grams. The difference between the two algorithms lies in the dissimilarity metric that is used. Stamatatos adds a measure based on the frequency norm of available training data by concatenating all of the training data from all of the authors in the dataset. By changing the distance measure, the algorithm can weight those n-grams that deviate the most from the norm to help determine the author of the system. If the n-gram exactly matches the norm, it would be weighted as a 0 since it does not contribute to identifying the author from other authors. This dissimilarity measure is shown in Algorithm 3 .

The Teahan algorithm [179] uses compression based methods for measuring the information content of messages to determine authorship styles. Teahan uses crossentropy based on the character n-grams of varying lengths, up to 5-grams, with respect to a category model. This cross-entropy method measures the average bits per coded symbol in the documents. Sundararajan [174] and Neal [89] found this algorithm, 


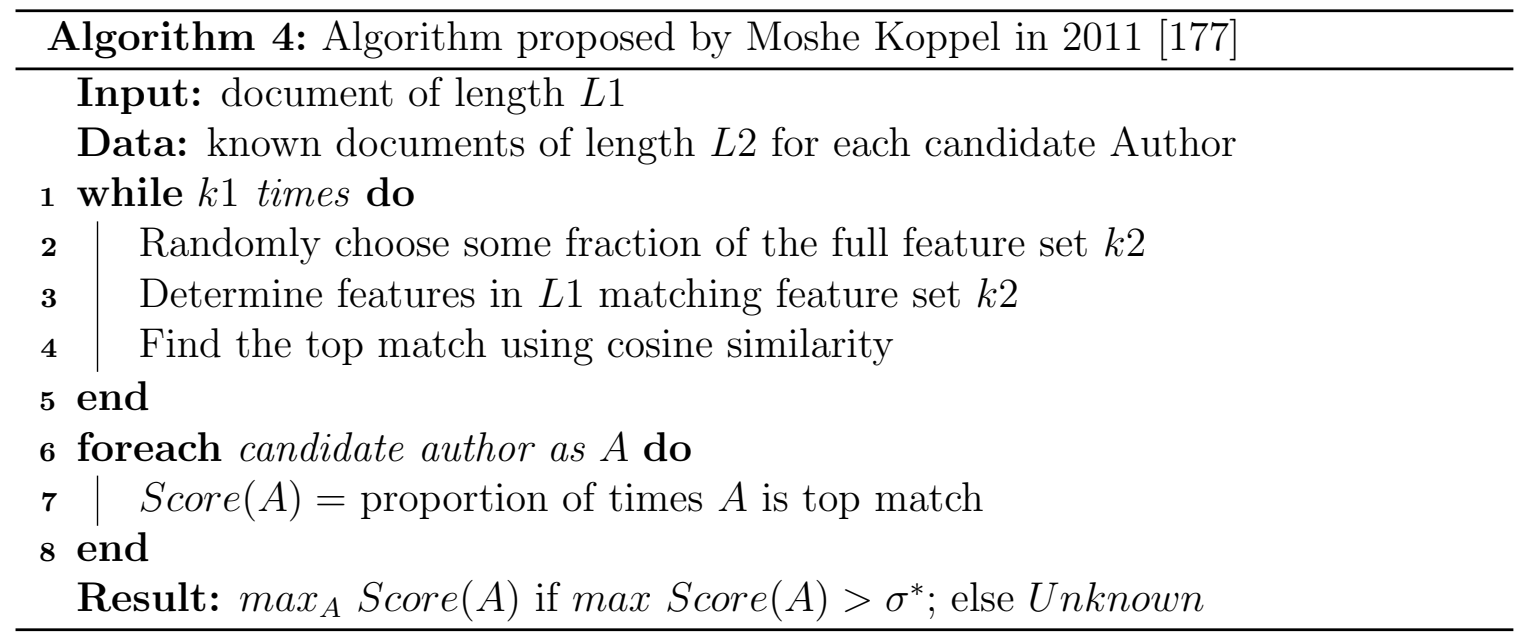

among the fourteen tested, to perform the best when document lengths were fixed. Benedetto [181], another compression algorithm using the LZ77 algorithm to measure document similarity also performed well in the testing. Because of this, compression based methods are interesting, but more understanding into how these algorithms function would need to be gained to understand if they are actually capturing stylistic information of the documents or matching based on topic and genre specific words.

The Koppel algorithm [177] was designed based on Koppel's prior findings in [182]. This algorithm assumes based on that finding that the known text of the author is likely to be the text most similar to the document even as the feature sets are varied. While another author may match on a handful of feature sets, it is unlikely that a non-match would stay consistent of many different features sets. The idea of this algorithm is therefore to check if a given author proves to be the most similar to the test for many different randomly selected feature sets of a fixed size. Koppel therefore proposes the algorithm shown in Algorithm 4.

Overall the findings of [174] and [89] shows that character level features appear to perform the best in large scale authorship attribution systems. However, the concept of style in writing samples is not well understood. The University of Florida is continuing to perform studies in an attempt to better understand the concept of writing styles in short documents, such as social media [183]. Authorship Attribution 
and Verification has been shown as a potential for continuous verification by the University of Florida [184, but in order to make such systems accurate enough to be used at a larger scale, a better understanding of the problem needs to occur.

\section{English Performance}

This dissertation reproduces the work conducted in [174 to determine the performance of the top-performing algorithms for selection in further experimentation. Due to computational limitations, the speed of the algorithms and memory efficiency of the algorithms are essential factors in the selection of the algorithms as well.

The CASIS dataset, discussed briefly in Chapter II] was used for testing. This dataset was also used in testing in both [174] and [89]. We follow the same processes outlined in their papers, breaking each author sample down into samples containing equal numbers of sentences, including 5,10 , and 20 sentences. While their original papers looked at two sentences per sample, it was shown that cutting the samples down this far degrades the algorithms' performance, likely due to some sentences being useless for stylometric analysis, which is explored in later studies [183]. This breakdown, based on the number of sentences, was achieved by ensuring that the new samples contained no overlapping sentences from the original documents.

With this breakdown, we have a total of 378 authors with 6,691 samples in the fivesentence dataset; 103 authors with a total of 1,597 samples in the ten-sentence dataset; and 19 authors with 229 Samples in the twenty-sentence dataset; the original dataset contains 1,000 authors with 4,000 samples. These breakdowns match the datasets

outlines in [174]. For each of the four tested algorithms, a 5 -fold cross-validation was performed using each of the datasets $(5,10,20$, and original). Recognition accuracy was measured as:

$$
\text { Accuracy }=\frac{T P+T N}{T P+T N+F P+F N}
$$




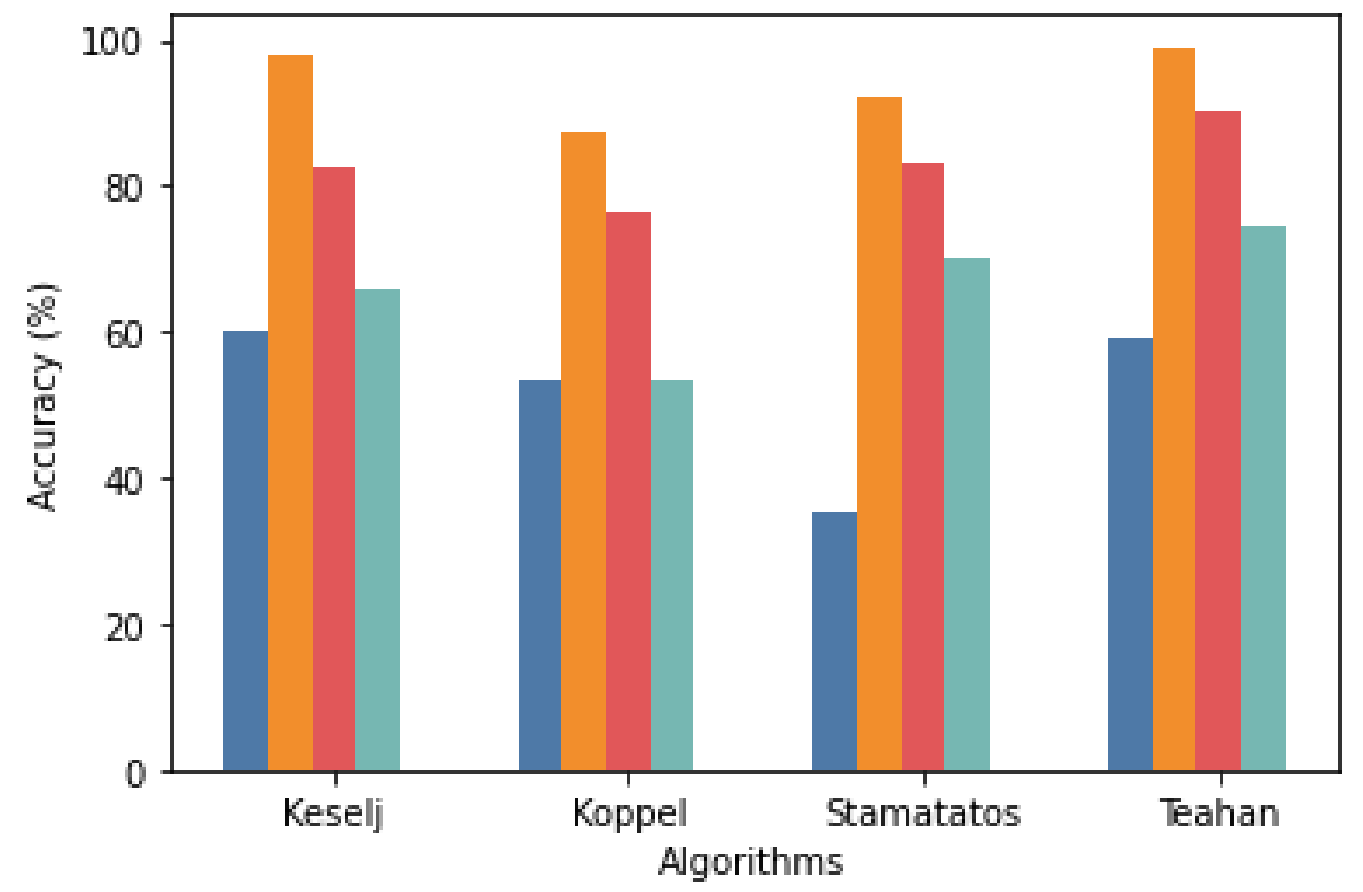

Original

Figure 14. Algorithm accuracy against the CASIS dataset for the four primary algorithms of focus: Keselj, Koppel, Stamatatos, and Teahan algorithms.

where $T P$ is True Positives, $T N$ is True Negatives, $F P$ is False Positives, and $F N$ is False Negatives. The accuracy of the four algorithms is shown in Figure 14.

The accuracy of the algorithms in this testing is comparable to the results achieved in [174] on the same CASIS dataset. Teahan performs slightly better than other systems, though all of the systems perform well on the experiments. Due to computational resources, we down-selected two algorithms based on the computational complexity of the Koppel algorithm and the memory resources required for the Teahan algorithm. On more massive datasets, the Computational complexity of the algorithm (and thereby clock time required) was far greater than the other algorithms. Running the algorithm on the Arabic dataset of 12,300 samples took about 15 minutes of processing per iteration. Future experiments rely on a large number of iterations, and even parallelizing the iterations on commodity CPUs would require months of processing. 
While the Teahan algorithm performs the best in the experiments, the amount of memory required far exceeds most commodity hardware and frequently crashed the python kernel with more massive datasets on commodity hardware, often exceeding the $24 \mathrm{~Gb}$ of memory used in experimentation for this dissertation on the Arabic dataset.

The Keselj and Stamatatos algorithms perform well based on their accuracy and are speedy algorithms, even on commodity hardware. Based on the results, we see that the Keselj algorithm performs slightly better on the CASIS datasets with about $60.4 \%$ accuracy on the Original dataset, compared to the $35.4 \%$ accuracy of the Stamatatos algorithm on the same dataset. The Stamatatos algorithm does perform slightly better on the five-sentence dataset, but the accuracy on the ten-sentence dataset is comparable, and the Keselj algorithm performs slightly better on the twentysentence dataset (97.8\% accuracy vs. $92.1 \%$ on the Stamatatos algorithm).

Because of the slightly better performance, further experimentation in this dissertation focuses only on the Keselj algorithm. Future research experiments will look at the performance impacts of translation on all four of the algorithms. Further analysis of the algorithms is performed. The False Acceptance Rate and False Rejection Rates are calculated and plotted on a ROC curve using Cosine similarity measure, shown in Figure 15. We see that based on the ROC curve, the algorithm does perform reasonably well, though the 20 sentence dataset does perform slightly better overall. Using these metrics, we can calculate the Equal Error Rates (EER) for each of the datasets. The average EERs for the datasets are: $23.8 \%$ for the original dataset, $25.7 \%$ for the twenty-sentence dataset, $27.4 \%$ for the ten-sentence dataset, and $33.0 \%$ for the five-sentence dataset.

Lastly, we can calculate the sensitivity of the keselj algorithm and plot the genuine vs. imposter distributions. Ideally, we would want to see that these distributions do not have significant overlaps. When there is little overlap, we can easily distinguish 


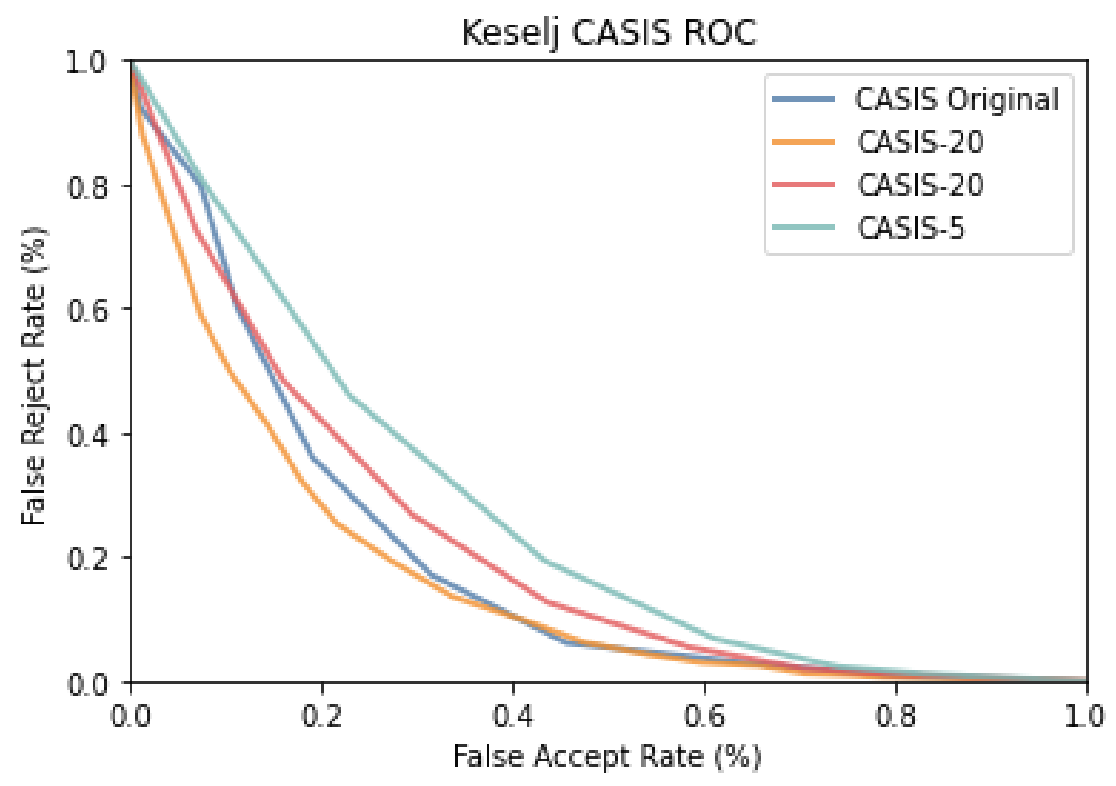

Figure 15. ROC Curve of FAR to FRR showing Curves for number of CASIS sentences.

genuine authors from imposter authors. However, as shown in Figure 16, we see that the keselj algorithm has a significant overlap. This overlap indicates that authorship attribution is a challenging problem, even in the English language. Calculating the Sensitivity index (d') against the datasets is also done: 0.923 for the original dataset; 1.258 for the twenty-sentence dataset; 1.213 for the ten-sentence dataset; and 1.106 for the five-sentence dataset. Overall, amongst all of the algorithms tested, holding the number of sentences constant improves the sensitivity index, ensuring that the algorithms can distinguish the genuine authors from the imposter authors. This finding shows that the Arabic and Chinese datasets were fixed to a 25 sentence sample minimum for each of the authors. 


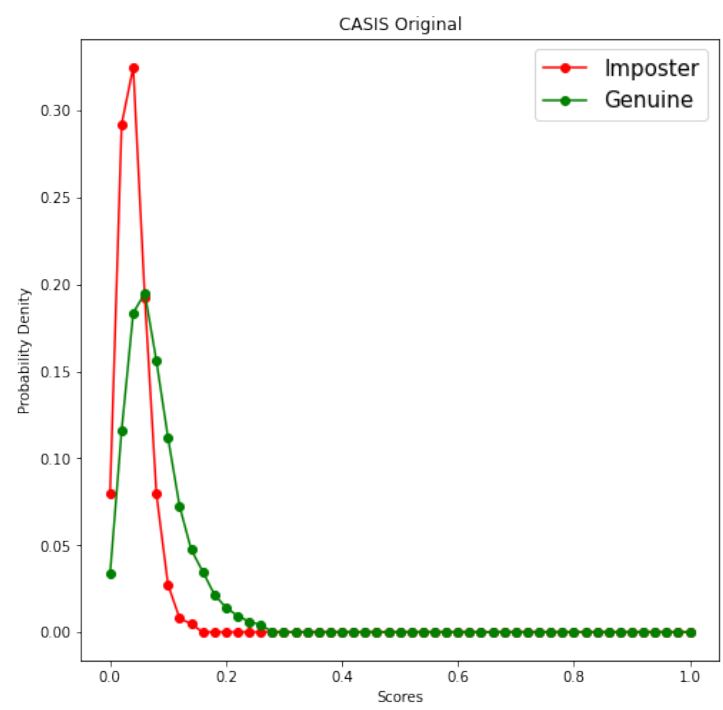

(a) CASIS Original

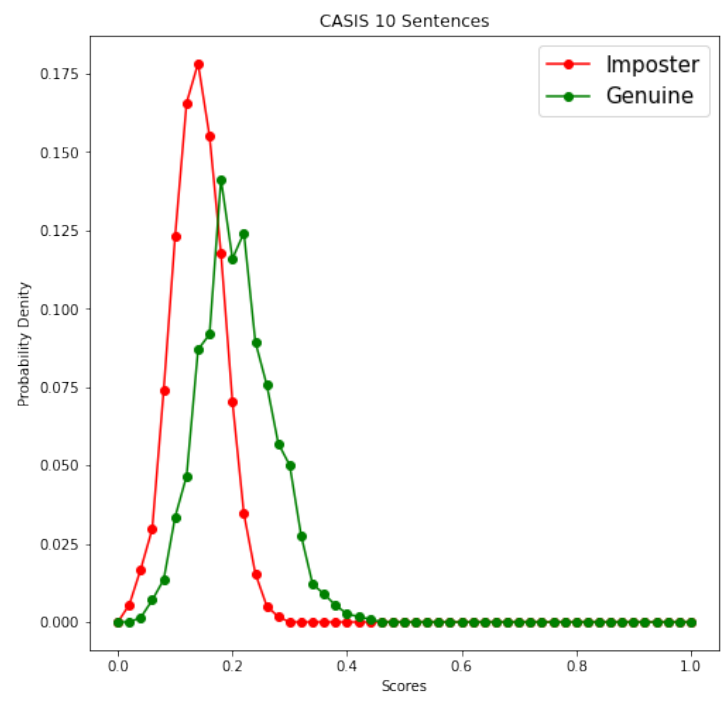

(c) 10 Sentences

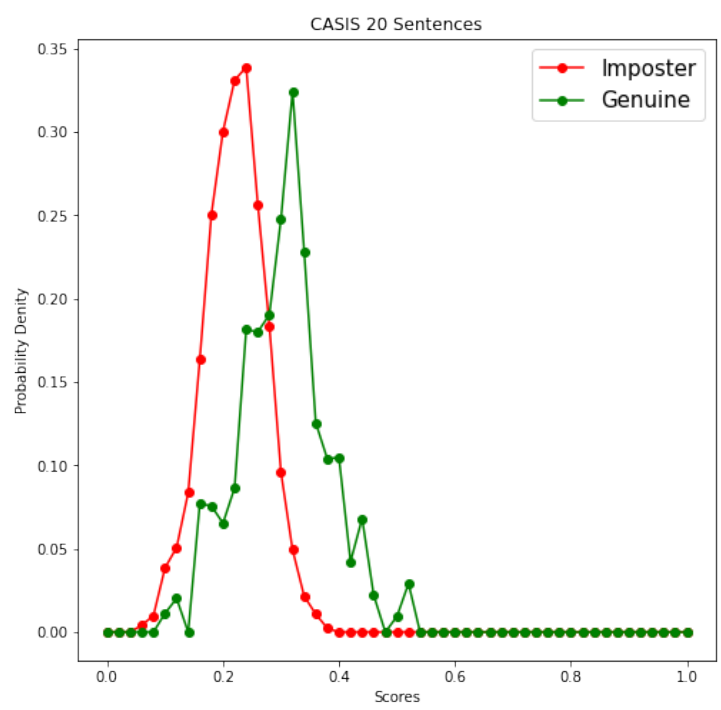

(b) 20 Sentences

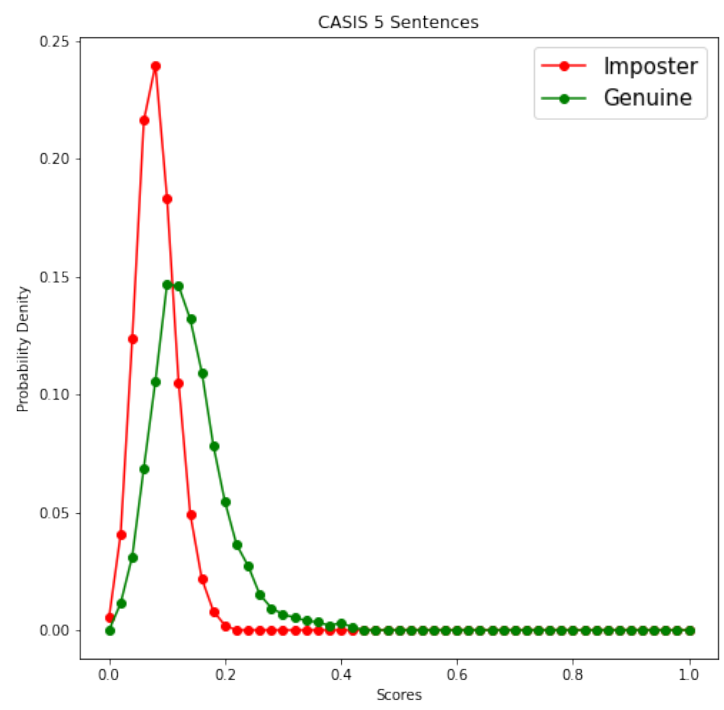

(d) 5 Sentences

Figure 16. The probability density charts for the Keselj algorithm showing the genuine vs imposter distributions within subsets of the CASIS dataset. 


\section{CHAPTER IV}

\section{EFFECTS OF TRANSLATION}

The datasets and algorithms used in experimentation were discussed in Chapters [II and III, respectively. These datasets allow for setting up the experimentation necessary to determine the effects of translations on the algorithms. To do this, we must first translate any of the foreign language datasets using a translation engine.

Both the Affect and Personality algorithms use regression models to estimate the scores from the text. We can use the error rates to test the null hypothesis for each emotion and personality trait. This null hypothesis is expressed in $\mathrm{H}_{0}$,

$\mathbf{H}_{\mathbf{0}}$ (Null hypothesis): There is no statistically significant difference between the means (or medians, in the case of any distribution except normal) of the distributions of the error rates for each of the emotions or five-factor model traits between the English language and translated text.

Authorship verification, on the other hand, uses different metrics as the system is not a regression model. We use the Equal Error Rate to determine the effect of translation on the behavioral biometric system. Future experimentation should look at the sensitivity index (d') to determine whether translations impact the sensitivity of the algorithms. 


\begin{tabular}{|c|c|c|c|c|c|c|c|c|}
\hline \multicolumn{3}{|c|}{ Languages } & \multicolumn{4}{|c|}{ Multi-lingual dataset } & \multicolumn{2}{|c|}{ Dataset (No EN) } \\
\hline ISO 639-1 & Language & Family & \# Authors & \# Posts & \# Posts (EN) & $\% \mathrm{EN}$ & \# Authors (No EN) & \# Posts (No EN) \\
\hline es & Spanish & Indo-European & 784 & 95456 & 25354 & $20.99 \%$ & 598 & 73990 \\
\hline $\mathrm{pt}$ & Portugese & Indo-European & 212 & 26107 & 5958 & $18.58 \%$ & 113 & 13556 \\
\hline ro & Romanian & Indo-European & 141 & 15807 & 2425 & $13.30 \%$ & 68 & 12322 \\
\hline fr & French & Indo-European & 174 & 20761 & 4150 & $16.66 \%$ & 22 & 3830 \\
\hline it & Italian & Indo-European & 103 & 17380 & 2687 & $13.39 \%$ & 25 & 3822 \\
\hline no & Norwegian & Indo-European & 29 & 4725 & 1267 & $21.14 \%$ & 146 & 18941 \\
\hline cs & Czech & Indo-European & 26 & 3961 & 620 & $13.53 \%$ & 44 & 5219 \\
\hline $\mathrm{pl}$ & Polish & Indo-European & 65 & 6934 & 1400 & $16.80 \%$ & 111 & 15211 \\
\hline tl & Tigrinya & Afro-Asiatic & 175 & 21614 & 12594 & $36.82 \%$ & 119 & 24881 \\
\hline Sv & Swedish & Indo-European & 147 & 29433 & 2965 & $9.15 \%$ & 82 & 13573 \\
\hline id & Indonesian & Austronesian & 115 & 19130 & 5172 & $21.28 \%$ & 21 & 2574 \\
\hline hu & Hungarian & Uralic & 32 & 3844 & 879 & $18.61 \%$ & 22 & 3015 \\
\hline de & German & Indo-European & 54 & 7391 & 1911 & $20.54 \%$ & 18 & 3393 \\
\hline $\mathrm{nl}$ & Dutch & Indo-European & 66 & 8307 & 2164 & $20.67 \%$ & 22 & 2408 \\
\hline sk & Slovak & Indo-European & 18 & 3393 & 357 & $9.52 \%$ & 39 & 7420 \\
\hline et & Estonian & Uralic & 21 & 2287 & 344 & $13.07 \%$ & 6 & 717 \\
\hline vi & Vietnamese & Austroasiatic & 27 & 2912 & 970 & $24.99 \%$ & 50 & 6166 \\
\hline fi & Finnish & Uralic & 48 & 9193 & 1251 & $11.98 \%$ & 15 & 1622 \\
\hline sl & Slovenian & Indo-European & 9 & 1130 & 318 & $21.96 \%$ & 41 & 4556 \\
\hline af & Afrikaans & Indo-European & 58 & 6563 & 2326 & $26.17 \%$ & 23 & 2472 \\
\hline hr & Croatian & Indo-European & 35 & 3743 & 729 & $16.30 \%$ & 41 & 5813 \\
\hline da & Danish & Indo-European & 50 & 6692 & 1066 & $13.74 \%$ & 15 & 1537 \\
\hline sq & Albanian & Indo-European & 5 & 337 & 167 & $33.13 \%$ & 2 & 150 \\
\hline so & Somali & Afro-Asiatic & 2 & 82 & 77 & $48.43 \%$ & 1 & 52 \\
\hline
\end{tabular}

Table 17. Information about the languages that were identified in the dataset, along with a basic counts for each of the two created datasets, including a multi-lingual (containing some percentage of English posts) and a No English dataset. Since the original collection was targeted towards English speakers, many of the languages of authors in the dataset are Indo-European Family languages.

\section{Data Translations}

Google translate [185] was chosen as the primary translation engine for testing the algorithms. While other translation engines provide similar services, Google translate maintains some of the highest Bi-lingual Evaluation Understudy (BLEU) scores across translation engines on challenging foreign languages, like Arabic [186]. BLEU is an algorithm used for evaluating the quality of machine-translated text from one language to another. The BLEU algorithm is one of the first algorithms to claim a high correlation with human judgments, and has remained one of the most popular metrics for estimating the quality of translations [187].

Along with the high BLEU scores across numerous languages, Google translate also has far more bi-directional language translation models (e.g., English $\rightarrow$ Arabic $\rightarrow$ English, English $\rightarrow$ Chinese $\rightarrow$ English), ensuring that the data preprocessing for the experiment could capture a far greater number of possible languages. Google 
translate also has a built-in system for detecting the language for translations. Both the large number of language models and the ability to automatically detect the language was especially important in the translation of the personality dataset, which included 24 unique languages.

Google has also built the tool into its Chrome browser to automatically translate foreign language pages to another language for their users. For cost savings, this feature was utilized by creating unique web pages for each of the author files and using JavaScript and a Python Flask server to automatically go through each web page, scroll the page to ensure chrome translated the entire web page to English, then captured the table and passed it back to the Flask server to save the file as XML. This XML file was then parsed and associated with each of the author's posts and respective metadata (including any associated scores).

\section{Affect Dataset}

The affect dataset was much smaller than the other datasets translated. Translation of this occurred directly through Google translate's cloud API, improving the likelihood of missing some of the translations using the web browser approach described. Since the entire dataset collected involved a single dialect, the API was easy to use and only cost $\$ 20$ to run all $15,000+$ collected tweets. Translation was accomplished by looping through each of the collected Arabic tweets and passing the text to Google translate. The response was recorded with the original text and the emotion scores to ensure that each translation was adequately associated with the annotated scores for further experimentation.

\section{Personality Dataset}

The 11,829 authors, who identified as having a non-English primary language, were separated from the rest of the dataset. Files created for these authors were trans- 
lated using the Google translation script. Google's translation engine automatically detected the social media post's language and attempted to translate the text from the author's primary language to English. Once all posts finished translation, a final validation check, using python's LangDetect library, was conducted to ensure that the final text was English. This validation step is necessary, as translation engines may skip some of the authors' posts, either intentionally or unintentionally, if the system could not process the text fast enough. As described in Chapter III, 1,500 words is the optimum for the developed personality estimation algorithm. During this validation phase, each of the author's posts was counted for their number of words to ensure that the minimum word threshold was present. After removing any authors that failed the translation and had too few words, 2,396 authors met all criteria for experimentation.

Further preprocessing broke the authors down by their primary languages. A count of the number of authors for each language is in table 17. During the validation phase, a handful of posts remained that still classified as English posts. The percentage of English posts in this dataset are in the last column of table 17 for each language. Since the dataset described in section II] was initially targeted towards English speaking countries, it is worth noting in the table that many of the foreign languages identified are of the Indo-European family of languages, especially Latin rooted languages.

Experiments were run on this multi-lingual dataset to test the impact on multilanguage speakers. However, for more in-depth experimentation, English posts were removed to run similar experiments on author posts that contained no English. After removing the English posts from the identified authors and validating the minimum criteria was met, only 1,644 authors remained in the "No English" dataset. The remaining language author counts are in table 17 under the columns "\# Authors (No EN)" and "\# Posts (No EN)." 


\section{Authorship Dataset}

The size of the authorship dataset was also cost-prohibitive and used the web page translation method described earlier in this section. Much like the personality data, an author file was created containing all of the social media posts associated with each author. This author file allowed for a dynamic web page to load the posts of each author. These web pages were then translated in the Chrome browser and an XML file written back to the Flask server. The XML files were processed and associated back to the original text and metadata for each of the authors' posts. This dataset was then further processed, as described in Chapter III.

\section{Affect Analysis}

Due to the smaller size of the English language emotion data sets, some challenges arose in analyzing the English language dataset's performance for comparison to the foreign language data set. Monte Carlo cross-validation (MCCV) helped to estimate the error distributions. $\mathrm{MCCV}$ is a common technique in statistical learning that splits the dataset into two subsets through a random sample without replacement. The model is then trained on one portion of the dataset and tested on the other dataset, commonly done in traditional cross-validation methods. This method is repeated to estimate the distributions [188, 189], minimally for $N^{2}$ iterations to get a distribution as close to cross-validation over the unique datasets [190]. This method improved results in chemometric domains [191] over the commonly used "Leave-oneout" method.

The algorithm was trained on the English Language tweets within the dataset, with a random holdout of 10 tweets. These ten tweets calculated the error rates for the algorithm. The number of runs was calculated using equation 4 , and the total number of samples was calculated using equation 5. Only four of the six Ekman 
emotions are tested due to the limitations of the SemEval-2018 dataset.

$$
\begin{gathered}
|R|=|P \backslash H| *|H| \\
|S|=|R| *|H|
\end{gathered}
$$

Where $R$ is the set of runs, $P$ is the original dataset, $H$ is the holdout set, and $S$ is the set of samples. || represents the cardinality, or length of the sets. \represents the set difference; in this equation, the set difference removes all of the holdouts, $H$, from the original dataset, $P$. Using the SemEval-2018 dataset, we can calculate the number of samples acquired using this technique using the equations. The calculation for each emotion is shown in equation 6. Using this MCCV method allows us to sample the model's error rates using a smaller set of data.

$$
\begin{array}{r}
\text { Anger: } 2089 * 10=20,890 \text { runs } * 10=208,900 \text { samples } \\
\text { Fear: } 2641 * 10=26,410 \text { runs } * 10=264,100 \text { samples } \\
\text { Joy: } 1906 * 10=19,060 \text { runs } * 10=190,600 \text { samples } \\
\text { Sadness: } 3066 * 10=30,660 \text { runs } * 10=306,600 \text { samples }
\end{array}
$$

This method was not necessary for the analysis of the Translated Foreign Language dataset. Since the dataset was collected similarly to the SemEval-2018 dataset, we treat this translation dataset as a subset of the English model trained on the entire SemEval-2018 dataset. This collection method means that each translated tweet can run through the English language model and the error rates observed without using the MCCV method. For testing purposes, any tweet within the Translation dataset with a value of zero for a specific emotion was omitted. This omission resulted in a total of 3,440 Anger tweets, 2,766 Sadness tweets, 870 Fear tweets, and 5,388 Joy 


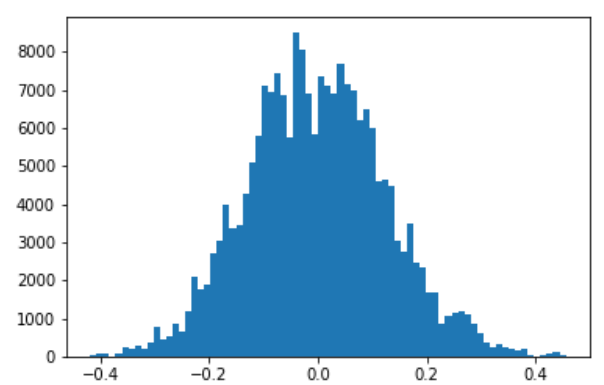

(a) Anger

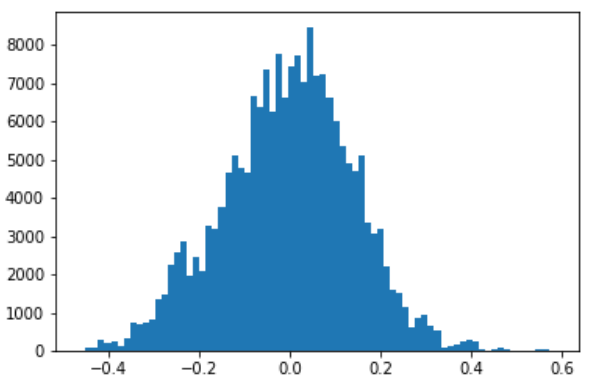

(c) Joy

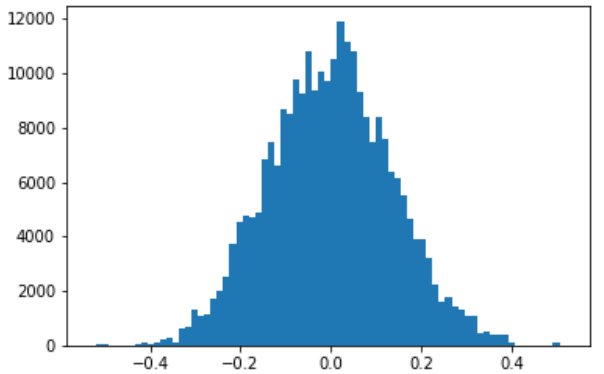

(b) Fear

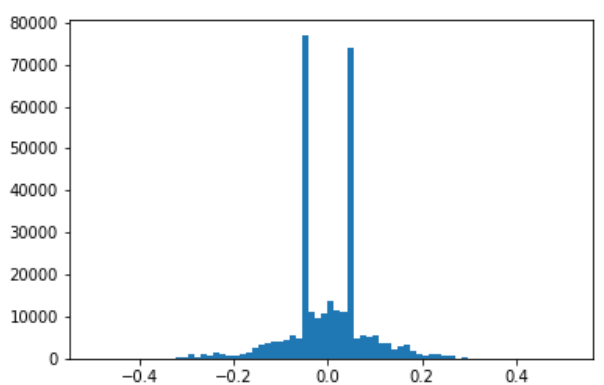

(d) Sadness

Figure 17. Error Distributions for each of the four tested emotions in English.

tweets within the translated dataset.

\section{Error Analysis}

The method for observing the English Dataset provides us with a sample of the Emotion algorithm's error distributions for each of the Four tested emotions. The squared error rate is calculated for each of the four tested emotions by subtracting the estimated value from the algorithm from the expected value of the annotations, then squaring the result. This error rate allows for the exploration of the impact of translations on the overall system.

Distributions of the English dataset for each of the emotions generally follows a normal distribution, except for the sadness distribution, which has unexplained peaks on both sides of the mean. These distributions are in Figure 17. The error squared distribution for the English dataset of all emotions shows an exponential distribution 


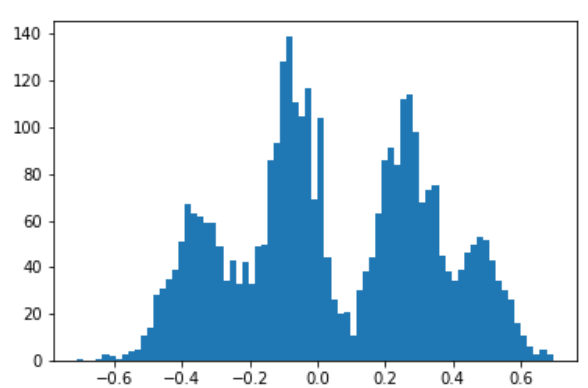

(a) Anger

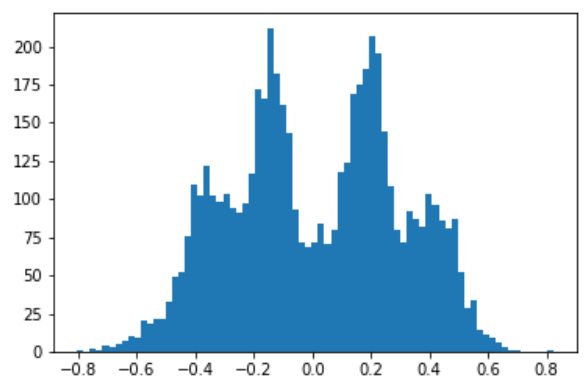

(c) Joy

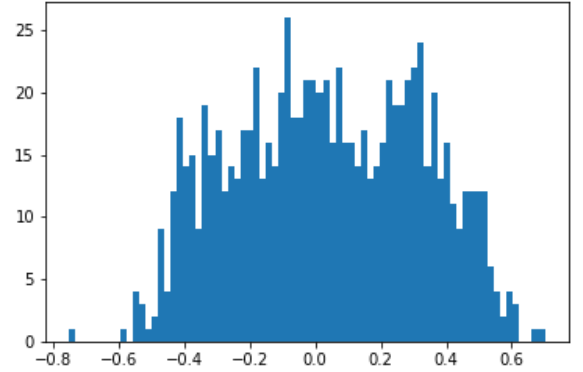

(b) Fear

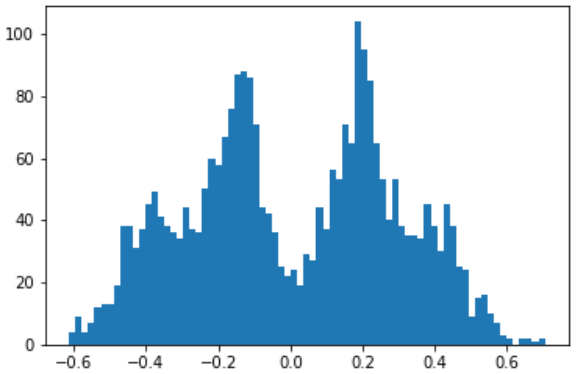

(d) Sadness

Figure 18. Error Distributions for each of the four tested emotions from translated Arabic.

19 .

The distributions of the Translated dataset were significantly altered through translations. The error rates, in Figure 18, obtained in the translation dataset are no longer normally distributed, but show multiple peaks within the distribution. This new distribution indicates an impact that can be observed by the error distributions of the two sets. The error squared distributions, in Figure 20, show exponential distributions, though the decay of the distribution is visibly much weaker than the English language dataset.

For our experimentation, comparisons of the error distribution can show whether the translations significantly alter the mean of the distribution in any direction. The error squared distribution helps identify any potential changes in the magnitude of the errors. 


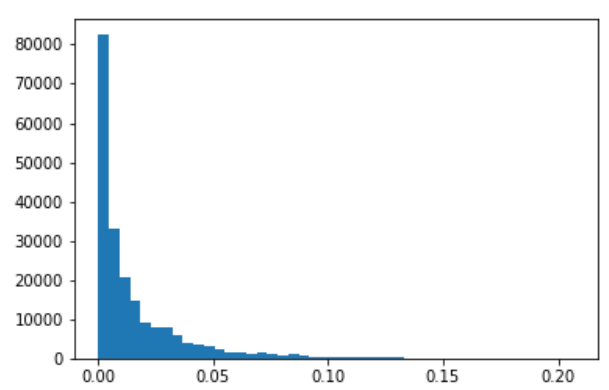

(a) Anger

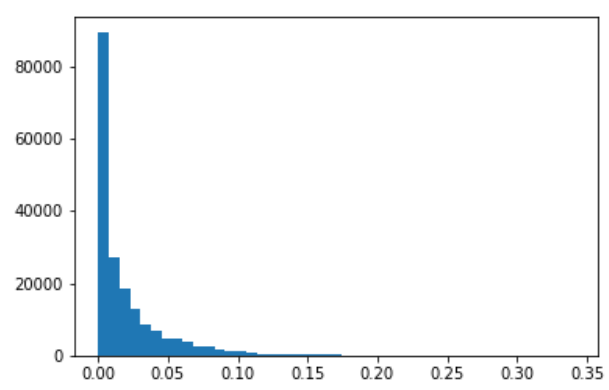

(c) Joy

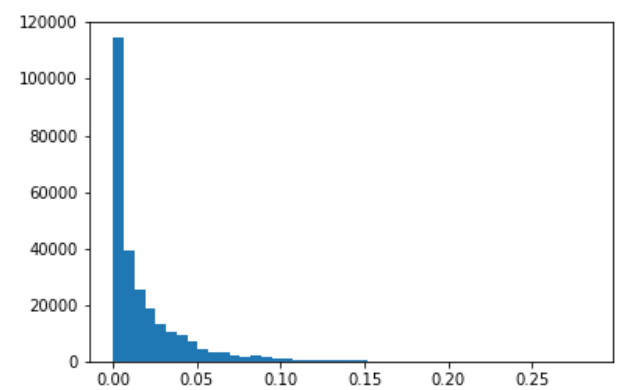

(b) Fear

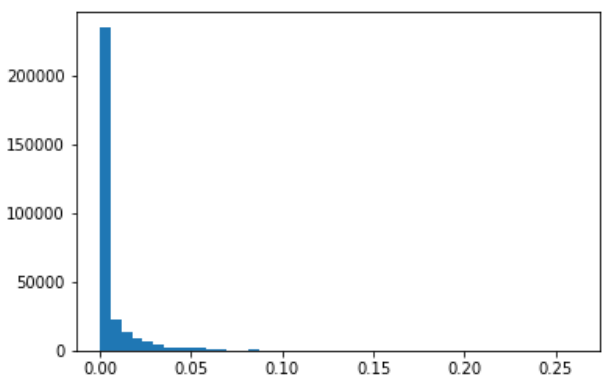

(d) Sadness

Figure 19. Error Squared Distributions for each of the four tested emotions in English.

\section{Statistical Analysis}

For affect analysis, we analyze both the error and the error-squared distribution to ensure that the observed histogram observations hold statistically. Each distribution for each of the emotions was tested using either a Student's t-test or the Mann-Whitney U-test. The error distributions for Anger, Fear, and joy are Normal distributions, so a Student's t-test is used to analyze the data. The sadness error distribution fails normality tests, so a Mann-Whitney U-test is used for comparisons.

The p-values of the t-tests for Anger, Fear, and Joy is significantly small. Analysis of the error distributions is shown in Table 18 . Anger shows a p-value of $1.93 * 10^{-22}$, far below a threshold of 0.05 . The Fear distribution has a p-value of 0.0001 , and joy has a p-value of $2.64 * 10^{-5}$. All three of the emotions with normal distributions reject the null hypothesis meaning that there is a statistically significant difference 


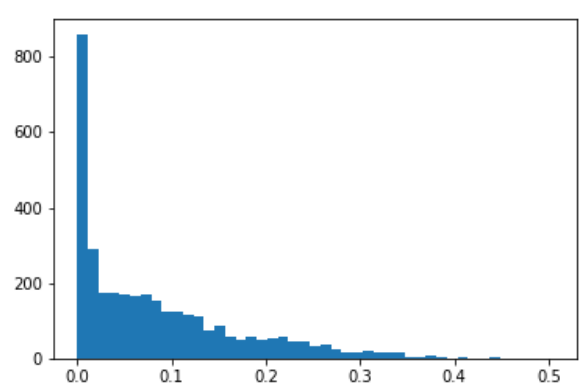

(a) Anger

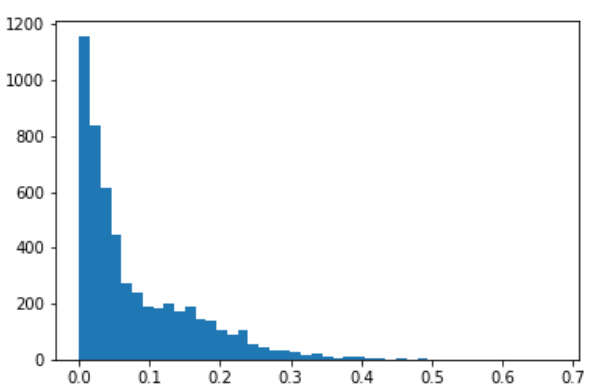

(c) Joy

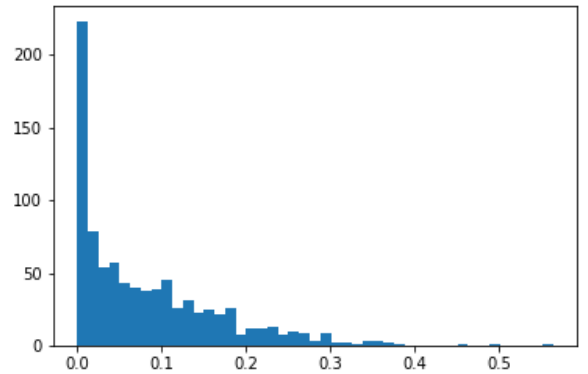

(b) Fear

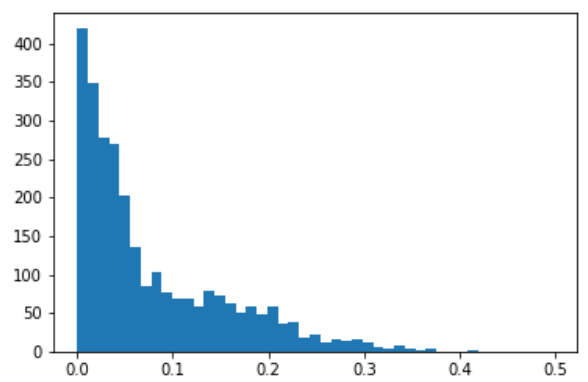

(d) Sadness

Figure 20. Error Squared Distributions for each of the four tested emotions from translated Arabic.

between the translated error distributions and the English error distributions. The Mann-Whitney u-test shows a very low probability and results in the machine giving a p-value of 0 . This error appears to be an issue with the way the computer handles floating-point numbers; since the p-value is very low, the floating-point numbers are not capable of storing the results, and the computer rounds the number to 0 .

We also run correlation analyses between the distributions, shown in Table 19. We see that Pearson's r p-values are above a 0.05 threshold, indicating that no significant correlations were found for the Anger, Fear, and Joy emotions. The Spearman $\rho$ correlation was also found to be insignificant for the sadness distribution. Given that the translation error distributions fail the normality test, the Cohen $\mathrm{d}$ metric for measuring the effect size is not appropriate.

The error squared distributions for all of the emotions follows an exponential 


\begin{tabular}{lrrcl}
\hline Feature & $\mathbf{u}$ tt-value & p-value & CI (mean [95\% CI $]$ ) & $\mathbf{H}_{\mathbf{0}}$ \\
\hline anger & -9.8128 & $1.9341 \mathrm{e}-22$ & $-4.8495 \mathrm{e}-02[-4.8505 \mathrm{e}-02$ to $-4.8485 \mathrm{e}-02]$ & Reject \\
fear & -3.8964 & 0.00010511 & $-3.7693 \mathrm{e}-02[-3.7711 \mathrm{e}-02$ to $-3.7676 \mathrm{e}-02]$ & Reject \\
joy & -4.2065 & $2.6351 \mathrm{e}-05$ & $-1.6425 \mathrm{e}-02[-1.6434 \mathrm{e}-02$ to $-1.6416 \mathrm{e}-02]$ & Reject \\
sadness & $8.22428 \mathrm{e}+07$ & 0 & & Reject \\
\hline
\end{tabular}

Table 18. Student's t-test results and p-values for each of the Emotions tested to determine whether any of the emotions are statistically significantly different between the Arabic dataset and the English Dataset. Sadness uses the Mann-Whitney U-test, since the sadness distribution fails the normal distribution test.

\begin{tabular}{lrrrr}
\hline Feature & Pear. r & Pear. p-val & Spear. Rho & Spear. p-val \\
\hline anger & 0.0106 & 0.5317 & 0.0127 & 0.4549 \\
fear & 0.0079 & 0.8166 & 0.0015 & 0.9655 \\
joy & 0.005 & 0.7136 & 0 & 0.999 \\
sadness & 0.0086 & 0.6511 & 0.0117 & 0.5383 \\
\hline
\end{tabular}

Table 19. Correlation Analysis for each of the Emotions tested to determine whether any of the emotions are statistically correlated in translations between Arabic dataset and English.

distribution. We use the Mann-Whitney U-test to compare the distributions between the translation and English datasets. This analysis is shown in Table 20. We see in each of the emotions that the p-values are significantly low. Anger, Fear, and Sadness error squared distributions result in a 0 . As was mentioned earlier, this appears to be caused by floating-point numbers being small enough that the computer rounds the value to 0 . The fear distribution has a p-value of $1.18 * 10^{-152}$. The resulting $\mathrm{p}$-values indicate that each error-squared distribution is statistically significantly different, and the null hypothesis is rejected. We also see that a correlation analysis using Spearman's $\rho$ results in no statistically significant correlations, as all p-values fall well above a threshold of 0.05 .

Since we know that the distributions are statistically significantly different, the medians show whether translations improve or degrade the algorithms' error. The median for each of the four emotions between the two datasets is compared in table 21 . We see in this table that all medians increase when the translated dataset is compared to the English dataset. Since these are exponential distributions, the magnitude 


\begin{tabular}{lrrrrrrr}
\hline Feature & $\mathrm{u}$-value & $\mathrm{p}$-value & Cohen d & Pear. $\mathrm{r}$ & Pear. p-val & Spear. Rho & Spear. p-val \\
\hline anger & $1.63827 \mathrm{e}+08$ & 0 & -1.0656 & -0.0087 & 0.6104 & -0.0188 & 0.2696 \\
fear & $5.55645 \mathrm{e}+07$ & $1.1825 \mathrm{e}-152$ & -1.0469 & 0.0502 & 0.1393 & 0.0049 & 0.8844 \\
joy & $2.26379 \mathrm{e}+08$ & 0 & -0.9647 & -0.0045 & 0.7401 & -0.0061 & 0.6534 \\
sadness & $8.22428 \mathrm{e}+07$ & 0 & -1.2537 & -0.0087 & 0.6463 & -0.013 & 0.4922 \\
\hline
\end{tabular}

Table 20. Mann-Whitney u-test results, p-values, and Correlation analysis for each of the Emotions' squared error tested to determine whether the magnitude of the errors are statistically significantly different between the Arabic dataset and the English Dataset.

\begin{tabular}{l|rr}
\hline Feature & English Median & Translated Median \\
\hline anger & 0.0075 & 0.0597 \\
fear & 0.0089 & 0.0557 \\
joy & 0.0090 & 0.0475 \\
sadness & 0.0025 & 0.0480 \\
\hline
\end{tabular}

Table 21. Median of the Error Squared distributions for each of the four tested emotions, showing that the median of the distributions for the translated dataset increase when using translations.

of the errors statistically significantly increases when translations are used in the system. This increase means that one can expect that the translations will result in statistically significantly increased error rates when translations are given to the algorithm.

\section{Personality Trait Estimation}

Various subsets of the Foreign Language datasets were able to be created from the personality dataset, including a Multi-Lingual set containing only the author's primary language and a handful of subsets to look at specific languages, and a subgroup focused on Linguistic Families. In this section, an analysis of the impact that translations have on the error rate is conducted, testing the hypothesis that Translations have a statistically significant effect on the estimation of personality traits. An analysis further breaks this down by looking at the error rate impact on a Multi-lingual set, sets containing only the author's primary language, a handful of languages that included only a single language (e.g., Spanish, French, and so forth), and finally looking 
at the linguistic family of the languages.

The section then digs into each of the 2,011 features used by the algorithm to determine whether translations have a statistically significant impact on each of the features' distributions. It finally explores the effect on the readability measures to examine whether the translated text has a statistically significant effect on the readability of text comparing English Language subsets and Foreign Language subsets.

A subset of the English data is used for experiments comparing the error rates for statistical significance against the foreign language datasets. This subset contains a list of primarily English speaking authors from the same dataset in Chapter II not used in training the personality estimation algorithm described in Chapter III. This subset allows us to compare sets of unobserved data to determine whether they likely come from the same distribution as one another.

Because the original dataset is large, a random sampling of the English authors is used to select the authors that most closely match the word count distribution of Non-English authors. A histogram showing the character counts of the Non-English speaking authors is in Figure 21a. Observations show that the number of characters the users use follows an exponential distribution with a median of 14,135 characters, a minimum character count of 5,923 , and a maximum character count of 125,794 .

English authors were randomly selected if they met the minimum character count matching the 5,923 characters observed in the non-English dataset. Figure 21b shows the distribution of the randomly sampled English dataset. This sampled dataset follows an exponential distribution with a median of 18,329 characters, a minimum character count of 5,295, and a maximum character count of 163,228.

With both a subset of English language speakers and the translations of the nonEnglish speakers as described in the section, estimates of the personality traits for each author in each of the datasets were calculated using the algorithm described in Chapter III, and calculate the error rates based on the expected, ground-truthed 


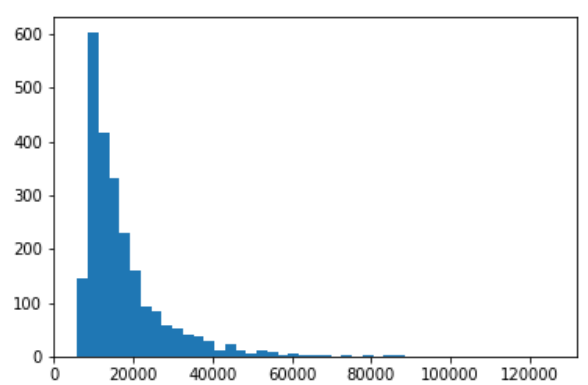

(a) Multi-Lingual

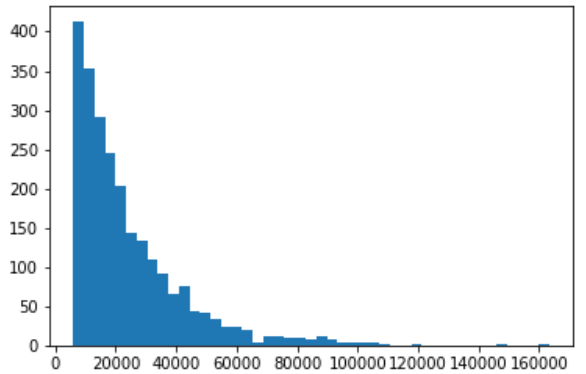

(b) English

Figure 21. Character counts for both the Multi-lingual dataset (a) and English language subsets (b).

values. Each of the resulting features used for determining trait values was stored for later analysis to understand how translations might be impacting the feature sets.

\section{Error Analysis}

Following the estimates of the author's personality traits, the squared error rate is calculated for each of the Five-Factor model traits by subtracting the estimated value from the expected value and squaring the result. The error rate is calculated for each of the datasets that are tested, including the English subset. This error rate allows us to explore what impact translations have in the system.

The English dataset distributions for each of the five traits follow a normal distribution, as shown in Figure 22. The error distribution shows the range of the errors without regard to the magnitude of the errors. This distribution allows us to understand whether the translations result in an overall change in the mean error expected from the algorithm. The squared error distribution of the English dataset follows an exponential distribution, as shown in Figure 23. This distribution shows the magnitude of the error. The medians of the distributions help determine whether the magnitude of the errors increase or decrease overall.

The distributions of the Translated Foreign Language datasets show the same dis- 


\begin{tabular}{lrrrrrrr}
\hline Feature & u-value & p-value & Cohen D & Pearson r & Pearson p-val. & Spearman $\rho$ & Spearman p-val. \\
\hline Error O & $1.75578 \mathrm{e}+06$ & 0.425248 & -0.0447 & -0.0063 & 0.791 & 0.0053 \\
Error C & $1.7253 \mathrm{e}+06$ & 0.134387 & 0.0434 & 0.0393 & 0.096 & -0.0107 & 0.8216 \\
Error E & $1.72238 \mathrm{e}+06$ & 0.116321 & 0.052 & 0.0014 & 0.9513 & -0.0453 \\
Error A & $1.67225 \mathrm{e}+06$ & 0.003442 & 0.0281 & -0.023 & 0.3292 & -0.0234 & 0.6515 \\
Error N & $1.62227 \mathrm{e}+06$ & $1.3 \mathrm{e}-05$ & 0.1703 & 0.0138 & 0.5577 & 0.0109 \\
\hline
\end{tabular}

Table 22. P-values for each of the Five Factor Model traits testing whether any of the traits are statistically significantly different between the Multi-lingual dataset and the English Subset.

tributions. The error rates for the five personality traits within the translated dataset have a normal distribution, as shown in Figure 24. The error squared distribution follows an exponential distribution, as shown in Figure 25.

Since the error distribution and error squared distribution types are similar between the two datasets, further analysis has to occur to determine whether translations significantly affect the results obtained by the algorithm. Since we care about whether the magnitude of the error significantly changes, we analyze the error squared distribution. Mann-Whitney U-tests are used to compare the error squared rates obtained between the English and translated distributions to test the null hypothesis, $\mathrm{H}_{0}$. The Mann-Whitney U-test is a non-parametric statistical method for testing whether two independent samples selected from populations share the same distribution [192, 193], much like the parametric Student's t-test is used for normal distributions.

\section{Multi-lingual Data}

The first dataset used was the multi-lingual dataset created in Chapter II. In this dataset, the authors who spoke a primary language other than English were identified. However, English posts were not excluded from those authors' writing samples, which allowed us to capture some of their English language texts. All of the non-English posts were translated into English. Using the translations and the English posts, the prediction algorithm was run to estimate their psychological traits, comparing this 


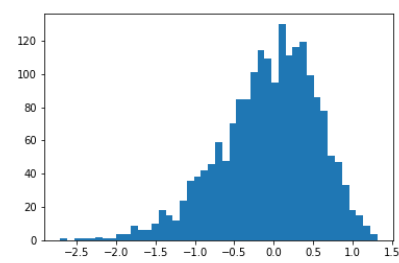

(a) Openness

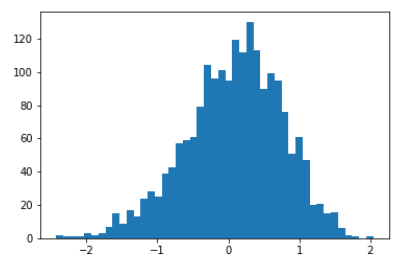

(d) Agreeableness

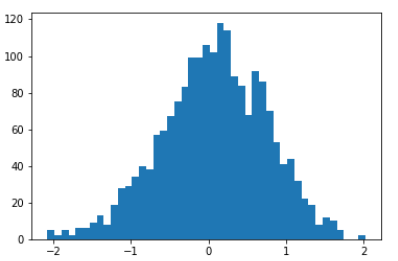

(b) Conscientiousness

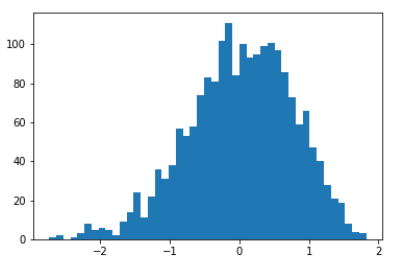

(c) Extraversion

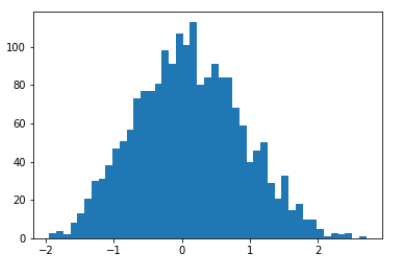

(e) Neuroticism

Figure 22. Error Distribution of the estimated OCEAN Traits for each of the 5 personality traits within the English language sample.

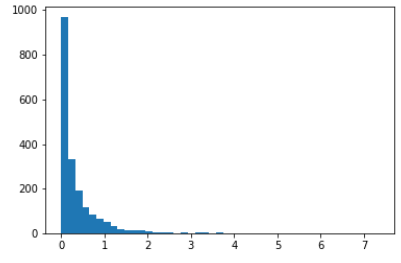

(a) Openness

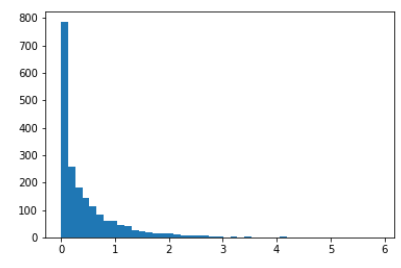

(d) Agreeableness

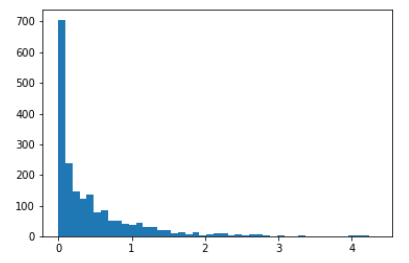

(b) Conscientiousness

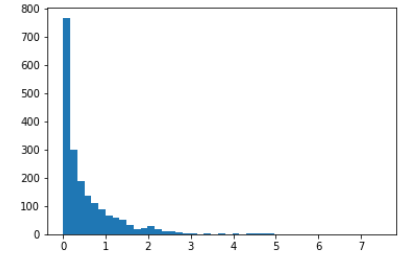

(c) Extraversion

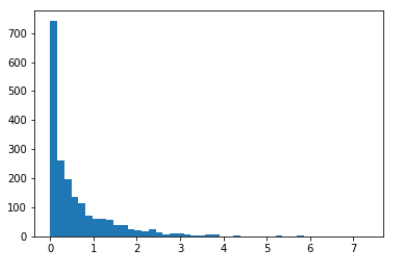

(e) Neuroticism

Figure 23. Error Squared Distribution of the estimated OCEAN Traits for each of the 5 personality traits within the English language sample. 


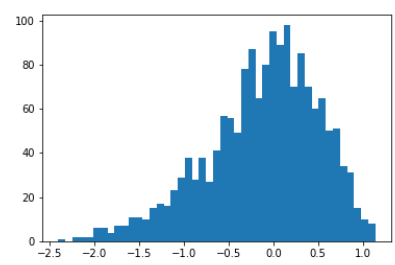

(a) Openness

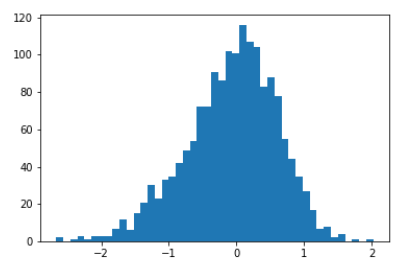

(d) Agreeableness

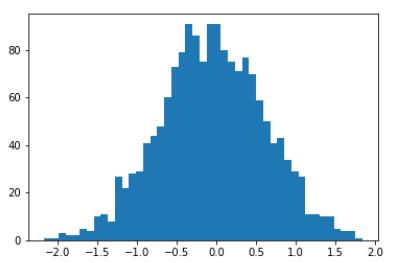

(b) Conscientiousness

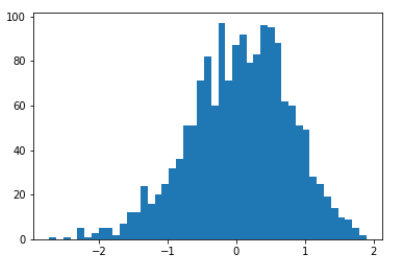

(c) Extraversion

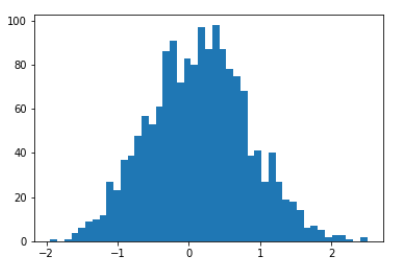

(e) Neuroticism

Figure 24. Error Distribution of the estimated OCEAN Traits for each of the 5 personality traits within the Translated sample.

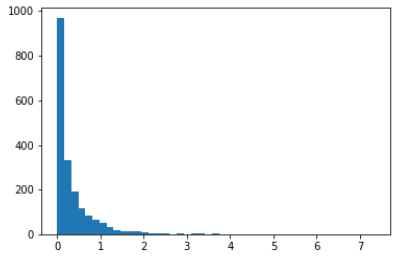

(a) Openness

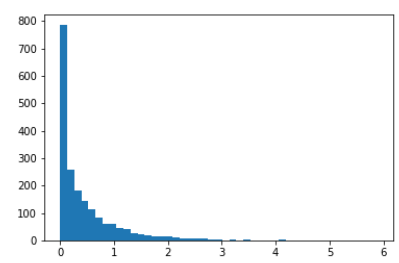

(d) Agreeableness

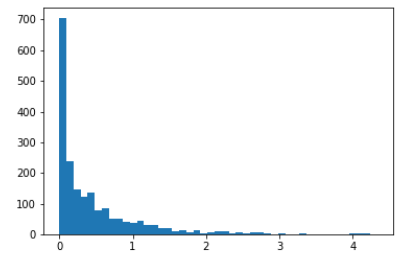

(b) Conscientiousness

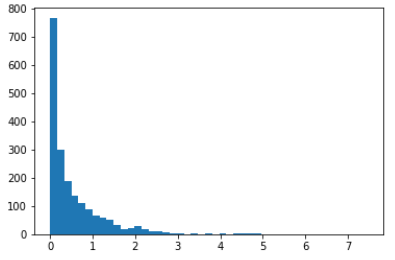

(c) Extraversion

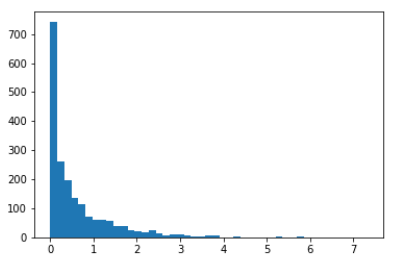

(e) Neuroticism

Figure 25. Error Squared Distribution of the estimated OCEAN Traits for each of the 5 personality traits within the Translated samples. 


\begin{tabular}{lrrrrrrr}
\hline Feature & u-value & p-value & Cohen D & Pearson r & Pearson p-val. & Spearman $\rho$ & Spearman p-val. \\
\hline Error O & $1.61004 \mathrm{e}+06$ & 0.4861 & -0.0594 & 0.0141 & 0.5678 & -0.0253 \\
Error C & $1.58432 \mathrm{e}+06$ & 0.1945 & 0.0412 & -0.0093 & 0.7059 & -0.0147 \\
Error E & $1.5772 \mathrm{e}+06$ & 0.1378 & 0.0621 & -0.006 & 0.8069 & 0.0089 \\
Error A & $1.54009 \mathrm{e}+06$ & 0.0112 & 0.0235 & 0.0044 & 0.8593 & -0.0129 & 0.5505 \\
Error N & $1.49992 \mathrm{e}+06$ & 0.0002 & 0.1687 & 0.0313 & 0.2048 & -0.0152 \\
\hline
\end{tabular}

Table 23. P-values for each of the Five Factor Model traits testing whether any of the traits are statistically significantly different between the Non-English Primary Language dataset and the English Subset.

error rate to the expected values in the original dataset.

The results of the Mann-Whitney U-test are in table 22, The null hypothesis cannot be rejected for some of the psychological traits, such as Agreeableness and Neuroticism. Both of these values are far below a threshold of 0.05. Based on this fact, the use of translations to estimate certain psychological traits significantly changes either the sample population or the population distribution, indicating that translations should not be used when calculating some of the psychological traits, even when English posts are present.

Openness shows a very high p-value, likely indicating that translations have no impact on estimating the openness score of a subject. While the remaining two traits, Conscientiousness, and Extraversion, are low, but not small enough to reject the null hypothesis.

\section{Primary Language}

Some English social media posts in the data could impact the results obtained, potentially raising or lowering the p-values in the initial tests. Further experiments conducted on the dataset removed all English language posts from the translation dataset. Outcomes of this experiment are in table 23 .

The results are consistent with the original test on the Multi-lingual dataset. The estimations for Agreeableness and Neuroticism remain statistically significantly impacted by the use of translations. In each of the remaining traits, the null hypothesis 
cannot be rejected. Openness remains high, while the p-values for Conscientiousness and Extraversion are only marginally increased.

\section{Per Language}

Since the datasets are not normal distributions, it becomes easier to test smaller subsets. Smaller subsets can be tested to determine whether specific languages might have a statistically significant impact on both the Extraversion and Conscientiousness traits. For this portion of the testing, only languages with a minimum author count of at least 50 authors meet the criteria in the "No English" dataset.

Results for the analysis are in table 24. Eight languages met the criteria, and test results for each of these languages are found in the same table. Depending on the language, certain traits jump between being statistically significant and not. We speculate that the resultant translation for each of the languages might have a considerable impact on the feature sets, causing the significance test to give such drastically different results.

Spanish, French, and Italian had by far the most significant number of authors. The results show that Openness and Extraversion are statistically significantly different from those of the English subset with p-values near or below a 0.05 threshold. Agreeableness and Neuroticism both have low p-values, but in each, one of the languages appears to flip the language from being statistically significant. Conscientiousness was only statistically significant for the Spanish language. Based on this experiment, it appears that the translation's language has an impact on the ability to estimate psychological traits.

\section{Per Linguistic Family}

Most of the languages identified as an author's primary language were Indo-European languages. It is also worth looking at each of the linguistic families for each of the 
languages. A clustering of those authors was completed based on linguistic families of their primary spoken language. For example, Indo-European includes Spanish, French, Italian, Portuguese, and Swedish. The Languages and their Linguistic families are in table 17.

The results of this portion of the experiment are in table 25. The analysis shows that the null hypothesis is not rejected for the Openness, Conscientiousness, and Extraversion traits (though it can be for Extraversion with Afro-Asiatic languages). The null hypothesis is rejected for Agreeableness and Neuroticism, except Austronesian languages and Neuroticism in Uralic languages.

\section{Impact on Feature Distributions}

The feature set for estimation is made up of 2,011 different features, as laid out in section III. Each feature is calculated for the "No English" and English datasets to determine the effect that translations have on features used by the algorithm. These features include the conditional probabilities of each of the 2,000 topics, along with the counts of negative sentiment, "hapax legomina", "dis legomina", and the Yule, Sichel, Brunet, and Honore measures of vocabulary richness. Each of these topics and the Natural Language Processing measures make up exponential distributions, so any testing on the impact that translations have on the feature sets must use the Mann-Whitney U-test.

The analysis of the effect on the feature distributions can be found in table A1. Only a handful of the tested features were acceptances of the null hypothesis, with many of the scores rejecting the null hypothesis having very low p-values, usually a smaller value than $10^{-100}$, representing a very significant difference. In total, 141 of the features tested accepted the null hypothesis meaning that they were not statistically significantly different. The remaining 1,870 features rejected the null hypothesis. Based on the number of features that rejected the Null Hypothesis, it can be seen 
that the use of translations significantly alters the usual topics in the text from the authors, as shown by the very low p-values. It also decreases the median scores for vocabulary richness in using all scoring measures - Yule's K-measure, Sichel's Smeasure, Brunet's W-measure, and Honore's R-measure. This decrease can also be seen by observing the median values, which are significantly lower in the translation dataset.

\section{Impact on Readability Measures}

Along with vocabulary richness, further experiments examined how translations impact the readability measures of the text in English and foreign languages. While these features are not used in the algorithm to determine personality traits, they can help increase understanding of what impact translations might have on the text itself to determine the possibility of using translations for psycho-linguistic tasks in the future.

Table 26 shows six of the most common readability measures. In all cases, the translations significantly increase the difficulty in interpreting the text. The median score for English text requires only a 9th-grade reading level using the Flesch Kincade Grade Level metric. Using translations requires a college-level education (16th grade).

In nearly all cases, the p values are minimal (around $10^{-200}$ ), indicating a significant increase in the readability measures' distributions. This result means that, beyond the 1,840 features that were statistically significantly altered, a significant difference is found in other common linguistic measurements. Therefore, translations are likely not very well suited for use when calculating psycho-linguistic traits, such as personality estimation. 


\section{Authorship Attribution}

Limitations in the final BOLT Arabic and Chinese datasets pose some challenges in determining the effects of translations on the Authorship Attribution system. Similar to the Affect Analysis experiments, Monte Carlo Cross-Validation (MCCV) is utilized to determine the effects of translations on the algorithms' performance. Unlike the Affect Analysis experiments, the use of a behavioral biometric requires matching authors to one another. In this case, both the translations and the original text are utilized to determine the performance between them.

The experiment controls as many variables as possible to determine the metrics, relying solely on the algorithm's performance. Since the Keselj algorithm is meant to work on any language, it can be run on the Chinese, Arabic, and both translation datasets without any modifications. This feature of the algorithm allows the datasets to be a control, ensuring that the results are focused on the effects of translations rather than minor changes to the algorithm.

A random holdout of authors and documents is generated for each iteration. To control potential issues caused by random selection twice, the holdout sets and training sets contain the same authors and documents during each iteration between the translation and original text. Due to computational resources, only 100 authors are selected for testing. These authors remain the same between the translation and the original language datasets. The holdout set contains 10\% of the authors (10 authors) and three documents per author, for a total of 30 documents in the holdout set. The remaining documents are used to train the Keselj algorithm during each iteration.

In our experiment, we calculate the number of iterations required using equation 7:

$$
|R|=|A| \times \frac{\left|D_{a}\right|}{\left|D_{a}^{h}\right|} \times n
$$


where $R$ is the set of iterations, $A$ is the author set, $D_{a}$ is the Document set per author, $D_{a}^{h}$ is the set of documents per author in the holdout set, and $n$ is the number of times we wish to select each of the documents.

Since both datasets used a subset of 100 authors, the number of iterations is: $100 *(25 / 3) * 10=8000$. Eight thousand iterations are run for both the Chinese and the Arabic datasets on the Keselj algorithm. The number of iterations increases the likelihood of selecting each document 10 times, though there is no way to ensure that each document is randomly selected ten times. During each iteration, both the Original language and Translated text are run through the system, and performance metrics are calculated.

\section{Arabic}

Running 8,000 iterations of the Arabic dataset against the Keselj algorithm provides a vector of performance metrics measuring the performance of both the translation and Arabic datasets. Performance metrics calculated during each iteration include the False Acceptance Rate (FAR), False Rejection Rate (FRR), and Equal Error Rate (EER). The vector of FAR and FRR values are used to calculate the mean FAR, mean FRR, the standard deviation of the FAR, and standard deviation of the FRR. These are then plotted to show the Mean Reciever Operator Curve (ROC), shown in Figure 26.

We see in this ROC that there is a noticeable difference between the two ROC curves. Since the FAR and the FRR are used in the ROC curve, better performance is obtained when the Area under the curve (AUC) is smaller - or the blue line (mean ROC) appears closer to the $[0,0]$ point on the charts. In this case, we can visually observe that the original text performs far better than the Translation data. This observation is confirmed by calculating the AUC for the mean ROCs: 0.1586 for the translated set, and 0.0890 for the original Arabic dataset. We see then that using the 


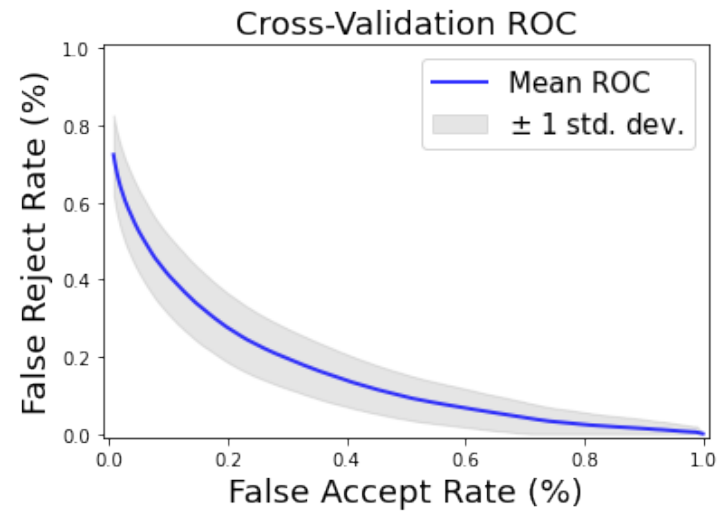

(a) Translation

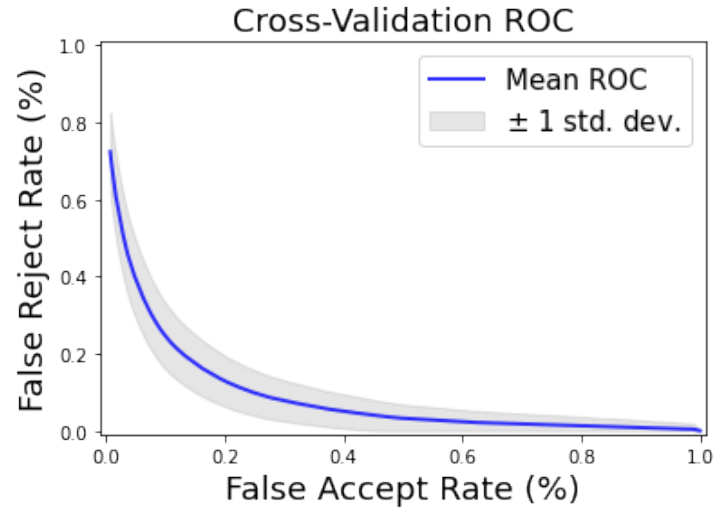

(b) Arabic

Figure 26. The Mean ROC Curves with Standard Deviation for both the Translated (a) and Original (b) Arabic datasets.

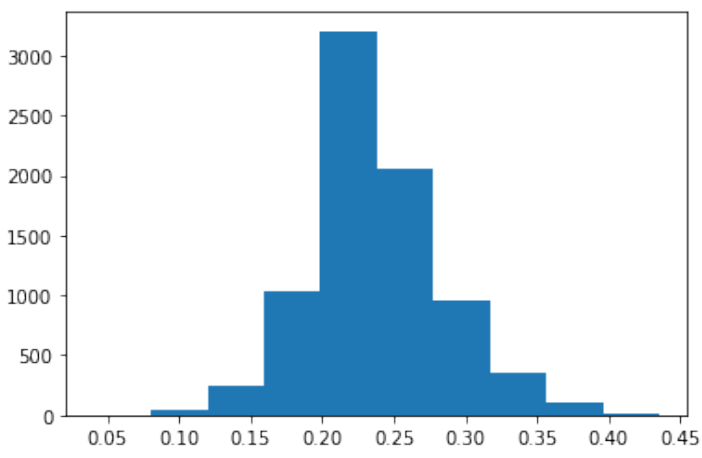

(a) Translation

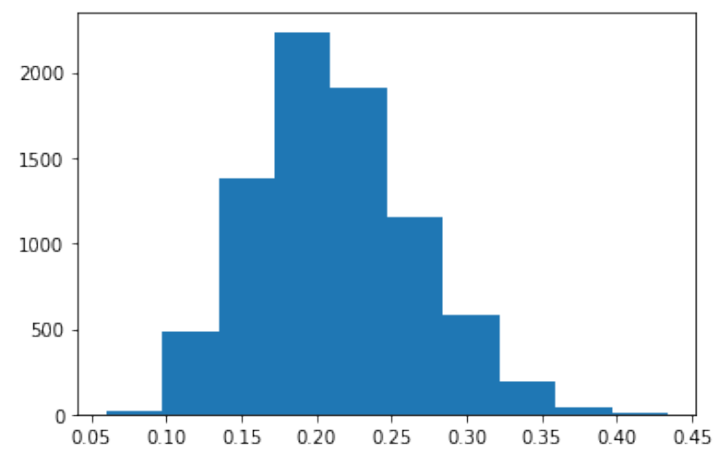

(b) Arabic

Figure 27. The distribution of EER obtained from the Translated (a) and Original (b) Arabic datasets.

original text provides far better performance than using Translations of the text.

The EERs are also calculated for each iteration. The calculations provide a vector of EERs that can be used to obtain a distribution of the EERs for the system using the dataset. A histogram showing the distributions of both the translations and the Arabic dataset is shown in Figure 27. We see, based on this, that the distribution appears to be normal. This normal distribution is confirmed by a D'Agostino $K^{2}$ test for normality: the translation dataset has a p-value of $1.062 \times 10^{-3}$, and the Arabic dataset has a p-value of $3.06 \times 10^{-13}$. Both fall far below a p-value threshold of 0.05 , indicating that the distributions are normal. 
Knowing that the distributions are normal, we can calculate some basic statistics for each of the distributions. The Basic statistics for both distributions are shown in Table 27. We see an increase in the average EER between the translation and the Arabic datasets, while standard deviations and variance remain close between the two distributions. A Student's t-test is used to determine whether there is a statistically significant difference between the two distributions.

The t-test results in a t-value of 26.8131 with a p-value of $5.92 \times 10^{-155}$, resulting in a rejection of the null hypothesis. This rejection means that there is a statistically significant difference between the two distributions. Since the mean of the Translation dataset is larger than the Arabic dataset, this means that there is a statistically significant increase in the EERs when translations are used.

We can use the Cohen d measure to determine the effect size of the observed phenomenon. Cohen d measure results in a score of 0.424 , a medium effect size between the distributions. Using Ruscio's Common Language conversion [194], there is a $61.8 \%$ chance that documents in the original language will provide a lower EER over the translated text. We also see statistically significant correlation coefficients. Pearson correlations result in a score of 0.6856 with a p-value close to 0 (floatingpoint rounding). A Spearman's $\rho$ results in a score of 0.6726 with a p-value close to 0 (floating-point rounding). These p-values mean that there is a strong positive correlation between the two distributions and that the system monotonically increases. As a random variable selected in the Arabic dataset increases in error, the translation distribution on the same document will never decrease.

\section{Chinese}

The same experiments were run on the Chinese dataset, running 8,000 iterations against the Keselj algorithm. The same performance metrics were calculated during each iteration of the experiment. The mean ROC curves for the Chinese and 


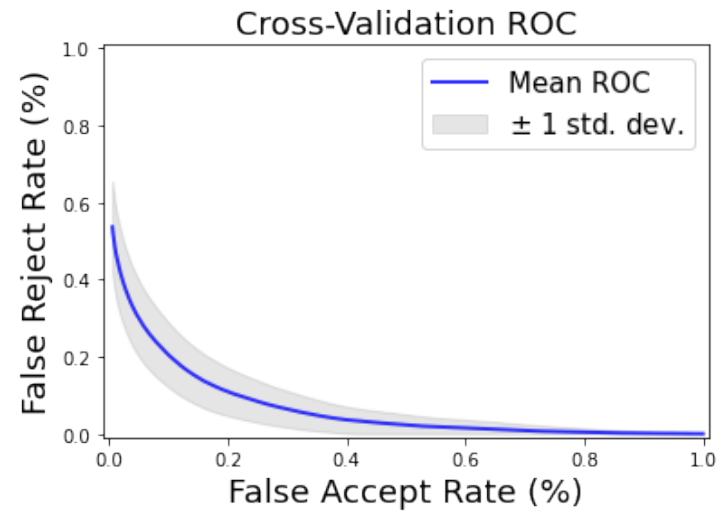

(a) Translation

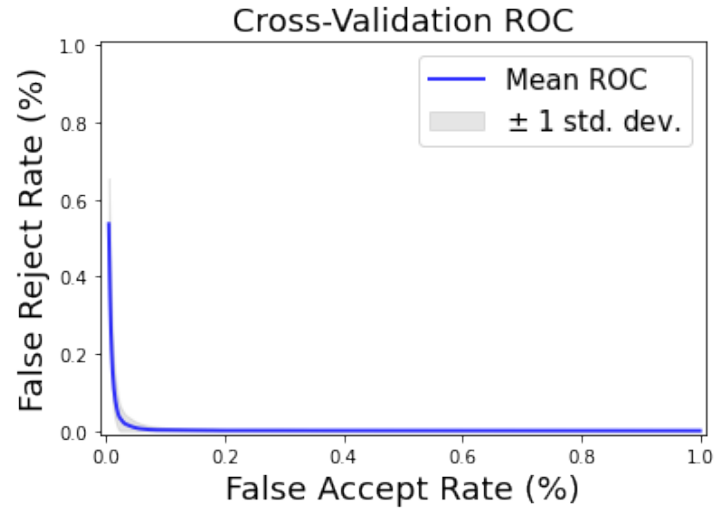

(b) Chinese

Figure 28. The Mean ROC Curves with Standard Deviation for both the Translated (a) and Original (b) Chinese datasets.

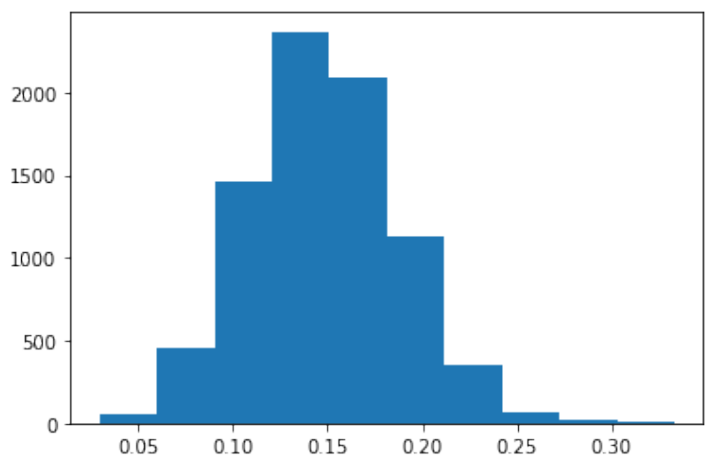

(a) Translation

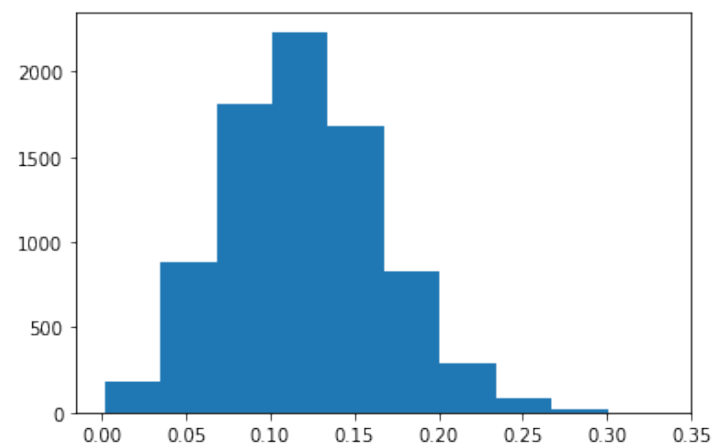

(b) Chinese

Figure 29. The distribution of EER obtained from the Translated (a) and Original (b) Chinese datasets.

Translated Chinese datasets are shown in Figure 28.

The differences in the ROC curves are more noticeably different between the two ROC curves. We again measure the FAR against the FRR, so better performance is obtained when the Area under the Curve (AUC) is smaller - the blue line (mean ROC) appears closer to the $[0,0]$ point in the charts. The performance is confirmed by measuring the AUC for the mean ROC for the translation, 0.0673, and the Original Chinese dataset, 0.0060. We see a significant performance increase when using Chinese logograms in the analysis. It is worth noting that the use of logograms appears to have an impact on the FAR and FRR. Further experimentation on Chinese char- 
acters against the Keselj algorithm could help understand the impact of logograms on the system's overall performance.

The EERs are calculated for each iteration, providing a vector of EERs, allowing for the determination of the EER distribution. A histogram showing the distributions for both the translations and the Original Chinese dataset is shown in Figure 29. Both distributions appear to be normally distributed. This visual observation is confirmed by a D'Agostino $K^{2}$ test for normality: the translation dataset has a p-value of $3.73 \times 10^{-15}$, and the original Chinese dataset has a p-value of $2.08 \times 10^{-24}$. Both of these distributions are well below a threshold of 0.05, indicating that the distributions are normal.

Basic statistics are calculated for each of the distributions and are shown in Table 28. We see an increase in the average EER between the translation and the Chinese datasets. The variance and standard deviations remain close between the two distributions. A Student's t-test is used to determine whether there is a statistically significant difference between the two distributions.

The t-test results in a t-value of 26.3506 , indicating a p-value of $7.83 \times 10^{-150}$ and rejects the null hypothesis. The rejection of the null hypothesis means that there is a statistically significant difference between the two distributions. Since the mean of the Translation dataset is larger than the Chinese dataset, there is a statistically significant increase in the EERs when translations are used.

Cohen $\mathrm{d}$ is again used to measure the effect size of the observed phenomenon. The Cohen $\mathrm{d}$ measure results in a score of 0.4166, a medium effect size between the two distributions. Using Ruscio's Common Language conversion [194], there is a $61.4 \%$ chance that a document's original language will provide a lower EER over the translated text. Statistically significant correlation coefficients are also observed. A Pearson correlation coefficient results in a score of 0.6546 with a p-value close to 0 (floating-point rounding). A Spearman's $\rho$ results in a score of 0.6481 with a p- 
value close to 0 (floating-point rounding). These p-values mean a moderately strong, positive correlation between the two distributions exists, and that the system is monotonically increasing. As a random variable selected in the Chinese dataset increases in error, the translation distribution on the same document will never decrease.

From the analysis of both the Arabic and Chinese datasets, it is observed that the use of translations in this behavioral biometric statistically significantly increases the error rates. When using a behavioral biometric, the analyst should avoid using translations in preference for the original language of the documents. 


\begin{tabular}{|c|c|c|c|c|c|c|c|c|}
\hline Feature & Language & U-value & p-val. & Cohen D & Pearson $r$ & Pearson p-val. & Spearman $\rho$ & Spearman p-val. \\
\hline \multirow{8}{*}{ Error O } & Spanish & 550616 & 0.0125 & -0.1461 & -0.0224 & 0.584 & 0.0546 & 0.1823 \\
\hline & French & 97502 & 0.0162 & 0.4151 & -0.0025 & 0.9792 & 0.0641 & 0.4998 \\
\hline & Italian & 59862 & 0.0767 & 0.3567 & -0.059 & 0.6325 & 0.0079 & 0.9493 \\
\hline & Portuguese & 138741 & 0.2702 & 0.0044 & 0.0005 & 0.995 & 0.0289 & 0.7293 \\
\hline & Tigrinya & 104222 & 0.2286 & 0.0642 & -0.0448 & 0.6409 & -0.0193 & 0.8404 \\
\hline & Swedish & 108092 & 0.0899 & 0.0921 & 0.0296 & 0.7489 & 0.0618 & 0.5041 \\
\hline & Indonesian & 76670 & 0.2403 & 0.1461 & 0.0796 & 0.477 & -0.027 & 0.8097 \\
\hline & Dutch & 42355 & 0.0505 & 0.3261 & -0.1087 & 0.4526 & -0.1712 & 0.2346 \\
\hline \multirow{8}{*}{ Error C } & Spanish & 557837 & 0.0372 & 0.0087 & -0.0295 & 0.4721 & -0.0276 & 0.5002 \\
\hline & French & 107722 & 0.3129 & -0.0018 & -0.056 & 0.5559 & 0.0226 & 0.8121 \\
\hline & Italian & 64446 & 0.322 & -0.0368 & -0.0358 & 0.7721 & -0.0466 & 0.7061 \\
\hline & Portuguese & 136854 & 0.1899 & -0.1593 & 0.0326 & 0.6959 & -0.0329 & 0.6935 \\
\hline & Tigrinya & 107825 & 0.4381 & -0.1073 & -0.0569 & 0.5533 & -0.0593 & 0.5367 \\
\hline & Swedish & 113958 & 0.3378 & -0.1375 & -0.0952 & 0.303 & -0.0754 & 0.4148 \\
\hline & Indonesian & 75219 & 0.1629 & 0.0169 & -0.1922 & 0.0837 & -0.1821 & 0.1015 \\
\hline & Dutch & 46168 & 0.2424 & -0.2887 & -0.0178 & 0.9024 & -0.057 & 0.6942 \\
\hline \multirow{8}{*}{ Error E } & Spanish & 561005 & 0.0567 & 0.0601 & 0.0447 & 0.2751 & 0.0291 & 0.4769 \\
\hline & French & 98705 & 0.0259 & 0.1539 & -0.0575 & 0.5451 & -0.0226 & 0.8119 \\
\hline & Italian & 59582 & 0.0685 & 0.1731 & 0.2371 & 0.0515 & 0.2051 & 0.0934 \\
\hline & Portuguese & 136103 & 0.1625 & -0.0316 & -0.0774 & 0.3533 & 0.0008 & 0.9926 \\
\hline & Tigrinya & 90501 & 0.0014 & 0.2866 & 0.0647 & 0.5002 & 0.0604 & 0.529 \\
\hline & Swedish & 100126 & 0.0047 & -0.2981 & 0.0015 & 0.9873 & -0.0214 & 0.8171 \\
\hline & Indonesian & 78384 & 0.3528 & 0.0729 & -0.0139 & 0.9013 & -0.0838 & 0.454 \\
\hline & Dutch & 48358 & 0.4371 & 0.1048 & 0.1476 & 0.3063 & 0.1048 & 0.4688 \\
\hline \multirow{8}{*}{ Error A } & Spanish & 568761 & 0.1372 & -0.0232 & -0.006 & 0.8828 & 0.0154 & 0.707 \\
\hline & French & 100920 & 0.0562 & 0.3468 & -0.0916 & 0.3348 & -0.0713 & 0.453 \\
\hline & Italian & 64237 & 0.3064 & -0.0045 & 0.1833 & 0.1347 & -0.2282 & 0.0612 \\
\hline & Portuguese & 123581 & 0.003 & 0.314 & -0.0514 & 0.5378 & -0.0086 & 0.9182 \\
\hline & Tigrinya & 98533 & 0.0473 & 0.312 & 0.0044 & 0.963 & -0.0483 & 0.6149 \\
\hline & Swedish & 114877 & 0.392 & 0.1317 & 0.007 & 0.9399 & -0.0345 & 0.7092 \\
\hline & Indonesian & 77516 & 0.2934 & 0.079 & -0.1014 & 0.3645 & -0.0835 & 0.4556 \\
\hline & Dutch & 47730 & 0.377 & 0.0633 & 0.0245 & 0.8659 & -0.0074 & 0.9591 \\
\hline \multirow{8}{*}{ Error N } & Spanish & 534660 & 0.0006 & 0.1668 & 0.0257 & 0.5306 & -0.0217 & 0.5956 \\
\hline & French & 104220 & 0.146 & 0.0214 & -0.1368 & 0.1484 & -0.1217 & 0.199 \\
\hline & Italian & 64428 & 0.3207 & -0.0718 & -0.0029 & 0.9811 & 0.1633 & 0.1834 \\
\hline & Portuguese & 134007 & 0.1003 & -0.063 & -0.1202 & 0.1483 & -0.0305 & 0.7145 \\
\hline & Tigrinya & 91306 & 0.0022 & 0.2276 & -0.144 & 0.1315 & -0.0227 & 0.8132 \\
\hline & Swedish & 116098 & 0.4673 & -0.1837 & 0.0618 & 0.5041 & 0.0419 & 0.6508 \\
\hline & Indonesian & 77870 & 0.3171 & 0.0126 & -0.1354 & 0.2253 & -0.19 & 0.0873 \\
\hline & Dutch & 45479 & 0.1925 & -0.1223 & 0.0918 & 0.526 & -0.0304 & 0.834 \\
\hline
\end{tabular}

Table 24. Mann-Whitney U-Test results for each feature in each of the languages that contained at least 50 distinct authors having enough non-English text and meeting the minimum criteria of 1500 words. 


\begin{tabular}{|c|c|c|c|c|c|c|c|c|c|}
\hline Feature & Linguistic Family & U-value & p-val. & Cohen D & Pearson $\mathrm{r}$ & Pearson & p-val. & Spearman $\rho$ & Spearman p-val. \\
\hline \multirow{4}{*}{ Error O } & Indo-European & $1.32 \mathrm{e}+06$ & 0.4538 & -0.0409 & 0.0425 & & 0.1181 & 0.0497 & 0.0677 \\
\hline & Afro-Asiatic & 104315 & 0.1883 & 0.0457 & 0.0356 & & 0.7096 & 0.0438 & 0.6467 \\
\hline & Austronesian & 76670 & 0.2403 & 0.1461 & 0.0796 & & 0.477 & -0.027 & 0.8097 \\
\hline & Uralic & 67345 & 0.1089 & 0.3234 & 0.2068 & & 0.0751 & 0.2201 & 0.0577 \\
\hline \multirow{4}{*}{ Error $\mathrm{C}$} & Indo-European & $1.30 \mathrm{e}+06$ & 0.1711 & 0.0258 & 0.0338 & & 0.2139 & 0.0158 & 0.5605 \\
\hline & Afro-Asiatic & 108977 & 0.4494 & -0.1091 & -0.029 & & 0.7617 & 0.0014 & 0.9885 \\
\hline & Austronesian & 75219 & 0.1629 & 0.0169 & -0.1922 & & 0.0837 & -0.1821 & 0.1015 \\
\hline & Uralic & 69854 & 0.2327 & -0.1513 & 0.108 & & 0.3562 & -0.0043 & 0.9708 \\
\hline \multirow{4}{*}{ Error E } & Indo-European & $1.31 \mathrm{e}+06$ & 0.3079 & 0.0252 & -0.0005 & & 0.9842 & 0.0302 & 0.2671 \\
\hline & Afro-Asiatic & 90582 & 0.0009 & 0.2852 & -0.0282 & & 0.768 & -0.0027 & 0.9772 \\
\hline & Austronesian & 78384 & 0.3528 & 0.0729 & -0.0139 & & 0.9013 & -0.0838 & 0.454 \\
\hline & Uralic & 67784 & 0.1262 & -0.1368 & 0.1629 & & 0.1625 & 0.1471 & 0.208 \\
\hline \multirow{4}{*}{ Error A } & Indo-European & $1.28 \mathrm{e}+06$ & 0.0307 & 0.0078 & 0.0236 & & 0.3857 & 0.0279 & 0.3047 \\
\hline & Afro-Asiatic & 100167 & 0.0597 & 0.3034 & 0.0884 & & 0.3539 & 0.0825 & 0.3873 \\
\hline & Austronesian & 77516 & 0.2934 & 0.079 & -0.1014 & & 0.3645 & -0.0835 & 0.4556 \\
\hline & Uralic & 62221 & 0.012 & 0.3423 & -0.1182 & & 0.3123 & -0.0859 & 0.4636 \\
\hline \multirow{4}{*}{ Error N } & Indo-European & $1.24 \mathrm{e}+06$ & 0.0006 & 0.1484 & -0.0088 & & 0.7474 & -0.0265 & 0.3305 \\
\hline & Afro-Asiatic & 92043 & 0.002 & 0.2238 & -0.1682 & & 0.0763 & -0.0899 & 0.3456 \\
\hline & Austronesian & 77870 & 0.3171 & 0.0126 & -0.1354 & & 0.2253 & -0.19 & 0.0873 \\
\hline & Uralic & 71125 & 0.3172 & -0.1671 & 0.0493 & & 0.6743 & -0.0235 & 0.8412 \\
\hline
\end{tabular}

Table 25. Mann-Whitney U-Test results for each feature in each of the Linguistic Families that contained at least 50 distinct authors having enough non-English text and meeting the minimum criteria of 1500 words.

\begin{tabular}{lrrrrcc}
\hline Feature & EN median & FL median & KL divergence & U-value & p-value & $\mathbf{H}_{\mathbf{0}}$ \\
\hline Flesch Kincade Grade & 9 & 16.8 & -7858.93 & $1.16 \mathrm{e}+06$ & $2.19 \mathrm{e}-234$ & Reject \\
Automated Readability Index & 10.9 & 22.3 & -9802.47 & $0.99 \mathrm{e}+06$ & $2.47 \mathrm{e}-292$ & Reject \\
Gunning Fog & 9.68 & 17.88 & -10485.7 & $1.04 \mathrm{e}+06$ & $8.47 \mathrm{e}-275$ & Reject \\
SMOG Index & 9.9 & 12.7 & -5593.67 & $1.13 \mathrm{e}+06$ & $6.63 \mathrm{e}-242$ & Reject \\
Coleman Liau Index & 7.54 & 9 & -2197.25 & $1.30 \mathrm{e}+06$ & $9.11 \mathrm{e}-195$ & Reject \\
Dale Chall Readability Score & 6.3 & 7.5 & -3058.69 & $1.15 \mathrm{e}+06$ & $4.28 \mathrm{e}-239$ & Reject \\
\hline
\end{tabular}

Table 26. Tests on the readability measures used to calculate the estimated education level to understand the text. We also test whether the differences between the English dataset and the translation dataset are statistically significantly different.

\begin{tabular}{lrr}
\hline Statistics & Translation & Arabic \\
\hline Average & 0.2382 & 0.2169 \\
Variance & 0.0024 & 0.0026 \\
Standard Deviation & 0.0494 & 0.0509 \\
Minimum & 0.0407 & 0.0598 \\
Maximum & 0.4355 & 0.4340 \\
\hline
\end{tabular}

Table 27. Basic statistics calculated against the Equal Error Rate distributions for both the Translations and the Arabic BOLT datasets. 


\begin{tabular}{lrr}
\hline Statistics & Translation & Arabic \\
\hline Average & 0.1470 & 0.1282 \\
Variance & 0.0018 & 0.0022 \\
Standard Deviation & 0.0429 & 0.0471 \\
Minimum & 0.0295 & 0.0017 \\
Maximum & 0.3332 & 0.3335 \\
\hline
\end{tabular}

Table 28. Basic statistics calculated against the Equal Error Rate distributions for both the Translations and the Chinese BOLT datasets. 


\section{CHAPTER V}

\section{CONCLUSIONS}

While it may seem intuitive that one should not use translations for estimating traits using psycholinguistics, it has been the author's observation that many data scientists, social scientists, corporations, and other entities have often disregarded the potential effects of translation on their systems. This dissertation provides insight into the impact of translations on three different psychological and behavioral biometric measures, including Personality Trait estimation, Emotion Analysis, and Authorship Attribution. The experiments outlined in this dissertation also provide experimental designs that various scientists should use to test the impact of translations on their systems before relying on translated text to make judgments about psychological traits.

While this dissertation is by no means comprehensive for the entire field, it has shown that in at least three measures of psychological traits, translations statistically significantly increase the systems' error rates. Translations of text were never meant to maintain the emotional or behavioral state of the original authors. Instead, its

purpose has been to convey the gist of a message to persons who cannot understand the original language. This dissertation does not intend to belittle Machine Translation research. Machine Translation still holds an essential place in protecting national security, improving business relations, and increasing globalization. This dissertation instead attempts to point out potential downfalls to other scientists who may rely 
on translation systems to provide insights that Machine Translation systems are not currently built to handle.

\section{Discussions and Speculations}

The experiments conducted on Personality Trait estimation show an analysis of the impact of translations to determine if translations can be used in such systems. The results are sporadic, often depending on the specific language being translated. Therefore, it is impossible to say when the use of translations allows for personality traits to be appropriately estimated. Each of the traits rejects the null hypothesis when specific languages are used in the experiment; however, the analysis shows that one can more reliably say that Agreeableness and Neuroticism are the most likely traits to have increased error rates when using translations.

It does appear that the use of translations from other Indo-European languages to English provides the opportunity to use translations for estimating Openness, Conscientiousness, and Extraversion as the Null hypothesis cannot be rejected based on the p-values. However, looking at each language indicates that this may depend on the language that is being translated. For example, Spanish rejects the null hypothesis for Openness, Conscientiousness, and Neuroticism, while not rejecting Extraversion or Agreeableness. French rejects the Null hypothesis for Openness and Extraversion, while not rejecting the null hypothesis for Conscientiousness, Agreeableness, or Neuroticism. Portuguese only rejects the null hypothesis for Conscientiousness.

Based on this, it appears to be dependent on the language model that is used in translation. It can be speculated that this is a remnant of how well a language can be translated (or the BLEU scores) from one language to another. For example, if we assume a language that is a one-to-one translation that mimics English grammatical and lexical structures, which could achieve a BLEU score of 1, translations likely would not impact the resulting estimation of personality traits. However, such a 
language does not currently exist, and BLEU scores rarely reach such scores. Such experiments may indicate the possibility of measuring the effect that a translation engine and translated language might have on the overall estimation of psychological traits, though further experimentation is necessary to prove such speculations.

The issues caused might also be caused by the effects of translations on the features that are being tested. The impact that translations have on the features tested in personality estimation is shown in Table A1. From this, we see that nearly all of the topics show a statistically significant difference between the translations and English language datasets. The fact that many of the features reject the null hypothesis indicates that translations have a detrimental effect on the topic distributions obtained from LDA. While the feature sets were not tested for each language, it can be speculated that each language has a unique impact on the topics discovered in each language as specific word choices from the translation engine might be preferred in their translations. Further experimentation around the Feature sets is necessary to discover how translations impact the system's topic model. The use of translations was also found to significantly impact the level of education required to comprehend the text, as indicated by various readability measures.

Emotion analysis experiments showed that in all cases for the four emotions tested, the emotions reject the null hypothesis with very low p-values. The low p-values indicate that the use of translations significantly increases, which can be observed between the histograms, which show a change in the distributions from normally distributed to non-normally distributed. We also find a lack of correlation between the translated and English language distributions. The lack of correlation indicates that translation appears the remove emotion words. In Machine Translation, the use of emotional adjectives likely adds little to the understanding of the message being conveyed and may get overlooked by the system.

Authorship attribution experiments resulted in the same effects. Since this was a 
behavioral biometric, the resulting study explored different metrics, and required the exploration of the algorithms in both the original language and translated documents. We see that translation statistically significant effects all of the metrics used in our experiments, including False Accept Rate, False Reject Rate, and Equal Error Rates.

\section{Impact}

The exploration of the impact of translations on these three systems indicates that translations should be avoided or tested for statistical significance in all systems that attempt to estimate psychological traits and behavioral biometrics that rely on linguistic analysis. The impact of these findings has a minimal impact on the overall uses of psychological tasks that might be useful in adding context or determining potential malicious persons, as described in Chapter \&. However, these findings show that each of these tasks should be done as close to the native language of the text as possible.

The performance of the Authorship attribution algorithms indicates that they are likely suitable for use in foreign languages. However, further experimentation should be done in understanding how effective the algorithms are when used on logogram based languages, such as hanzi (Chinese characters), instead of alphabetic languages (such as English and Arabic). The initial results in our exploration of the Keselj algorithm shows that such logogram based languages may be efficient.

However, research efforts should be increased to research and build natural language processing technologies that work in other languages. Much of the research efforts that have been found are primarily based on English language text. As globalization of economies increases and National Security threats expand worldwide for all nations, the importance of natural language processing technologies will be an essential aspect for identifying and contextualizing potential threats.

Identifying radical religiously motivated terrorists around the globe has given rise 
to the need for Arabic language tools. Some tools were explored in Chapter IV, such as Stanford's CoreNLP, Columbia University's MADAMIRA, and University of Maryland's Arabic toolkit provides some attractive opportunities to explore Arabic for Emotion analysis. Initial exploration of Arabic emotion analysis showed that the tools have promise, but the preprocessing of Arabic using CoreNLP was not nearly as useful as the English language tools. Further exploration of MADAMIRA and the University of Maryland's Arabic toolkit may provide better results, but further exploration in Natural Language toolkits for twitter processing in various languages would prove beneficial. The use of native language toolkits would alleviate any need for translations to estimate psychological traits.

\section{$3 \quad$ Future Research Efforts}

This dissertation is meant to show that the effects of translations may be detrimental to the estimates that are being made by psychological tools. However, there is still much work that should go into understanding those effects to determine whether translations can work, when they fail, and how to conduct psychological trait estimates in native languages.

A significant difference is shown in the histograms for the Root Mean Squared Error for emotion analysis, but little was done to understand the underlying effect on the feature distribution. Further experimentation could provide insight into how translations affect the distributions of features between two sets of data. These effects could allow for a complete understanding of how translations impact the algorithms, allowing further development of the algorithms to consider translations, making them more useful.

Additionally, preprocessing methods, or exploration of other morphological and lexical toolkits (such as MADAMIRA and the University of Maryland Arabic Toolkit) might provide improved overall analytic capabilities when processing Native Language 
text. While this would have no impact on the effect that translations have on the system, it would show the capabilities of the tools in Native Languages for additional comparisons.

Further experimentation on a linguistically homogeneous dataset needs to be conducted to determine whether the algorithm presented works in the native language. However, given the results of this experimentation, we strongly recommend building distinct models for individual languages to ensure that one can achieve the most accurate estimates possible in foreign languages. If translations must be used, scientists should conduct a full analysis of the impact their chosen translation system has on their estimates to determine which traits are more significantly affected.

A more comprehensive look at the languages that are being translated may provide insights into when translations fail, and whether traits can be more accurately detected in other languages. For example, rather than translating from Spanish to English, perhaps translations from Spanish to Portuguese may provide more accuracy. These types of studies, compared to the BLEU scores for translations from one language to another, could measure the potential impact that translations have on psychological estimates. However, this experimentation will require significant investments in building datasets across many different languages.

If there are specific languages that are less affected by translations, it would be worth exploring the translation of text to those languages and testing the systems using that language. Perhaps this could indicate that there is an intermediary or constructed language that could capture each language's nuances. By translating to this constructed language, perhaps using each of the systems on this constructed language could improve results across all languages.

Given the experimentation results in this dissertation, it is highly recommended to avoid the use of translations whenever the highest levels of accuracy are necessary, for instance, for National Security and Law Enforcement purposes. There is still 
much potential experimentation needed to understand the impacts of translations further, but building distinct models for individual languages can ensure that analysts and scientists achieve the most accurate estimates possible in foreign languages. If translations must be used for any reason, scientists should conduct a full analysis of their chosen translation system's impact on their estimates to determine which traits are more significantly affected. This analysis will help ensure that analysts and scientists are better informed of the potential inaccuracies and change any resulting decisions from the data accordingly. 


\section{REFERENCES}

[1] IBM, "Insider Threat Detection." [Online]. Available: https://www.ibm.com/ security/security-intelligence/qradar/insider-threat

[2] — " "IBM Watson: Personality Insights." [Online]. Available: https: //www.ibm.com/watson/services/personality-insights/

[3] G. Skloot and D. D'Agostino, "Introducing Personality AI," Crystal Knows, Tech. Rep., 2020. [Online]. Available: https://www.crystalknows.com

[4] J. Feldman, "Artificial Intelligence in Cognitive Science," in International Encyclopedia of the Social \& Behavioral Sciences. Elsevier, 2001, pp. 792-796. [Online]. Available: https://linkinghub.elsevier.com/retrieve/pii/ B0080430767016132

[5] K. D. Forbus, "AI and cognitive science: The past and next 30 years," Topics in Cognitive Science, vol. 2, no. 3, pp. 345-356, 2010.

[6] Raconteur, "A Day in Data," 2019. [Online]. Available: https://res.cloudinary. com/yumyoshojin/image/upload/v1/pdf/future-data-2019.pdf

[7] American Psychological Association, "2012 APA state licensing board list [Unpublished special analysis]," American Psychological Association, Washington, D.C., Tech. Rep., 2012.

[8] R. K. b. A. W. Khan, "Why Do We Need More Clinical Psychologists?" The Malaysian Journal of Medical Sciences, vol. 15, no. 2, pp. 1-2, 2008.

[9] A. W. Burgess, M. Mahoney, J. Visk, and L. Morgenbesser, "Cyber Child Sexual Exploitation," Journal of Psychosocial Nursing and Mental Health Services, vol. 46, no. 9, pp. 38-45, 9 2008. [Online]. Available: http://www.healio.com/doiresolver?doi=10.3928/02793695-20080901-01

[10] J. W. Patchin and S. Hinduja, "Bullies Move Beyond the Schoolyard: A Preliminary Look at Cyberbullying," Youth Violence and Juvenile Justice, vol. 4, no. 2, pp. 148-169, 2006.

[11] L. S.-L. Chen, H. H.-J. Tu, and E. S.-T. Wang, "Personality Traits and Life Satisfaction among Online Game Players," CyberPsychology 85 Behavior, vol. 11, no. 2, pp. 145-149, 4 2008. [Online]. Available: http://www.liebertpub.com/doi/10.1089/cpb.2007.0023 
[12] K. S. YOUNG, "Internet Addiction: The Emergence of a New Clinical Disorder," CyberPsychology \& Behavior, vol. 1, no. 3, pp. 237-244, 11998. [Online]. Available: http://www.liebertpub.com/doi/10.1089/cpb.1998.1.237

[13] A. Dhir, S. Chen, and M. Nieminen, "Predicting adolescent Internet addiction: The roles of demographics, technology accessibility, unwillingness to communicate and sought Internet gratifications," Computers in Human Behavior, vol. 51, pp. 24-33, 10 2015. [Online]. Available: https: //linkinghub.elsevier.com/retrieve/pii/S0747563215003519

[14] C. Ko, J.-Y. Yen, C. Yen, C. Chen, C. Weng, and C. Chen, "The Association between Internet Addiction and Problematic Alcohol Use in Adolescents: The Problem Behavior Model," CyberPsychology \& Behavior, vol. 11, no. 5, pp. 571-576, 10 2008. [Online]. Available: http://www.liebertpub.com/doi/10.1089/cpb.2007.0199

[15] S. Stieger, T. Eichinger, and B. Honeder, "Can Mate Choice Strategies Explain Sex Differences?" Social Psychology, vol. 40, no. 1, pp. 16-25, 1 2009. [Online]. Available: https://econtent.hogrefe.com/doi/10.1027/1864-9335.40.1.16

[16] E. von Zagorski, "Gender and modification of self-traits in online dating: The impact of anonymity, social desirability, and self-monitoring," Ph.D. dissertation, Walden University, 2013.

[17] J. T. Hancock, C. Toma, and N. Ellison, "The truth about lying in online dating profiles," Conference on Human Factors in Computing Systems - Proceedings, no. May 2014, pp. 449-452, 2014.

[18] D. Cappelli, A. Moore, R. Trzeciak, and T. J. Shimeall, "Common sense guide to prevention and detection of insider threats 3rd edition-version 3.1," Software Engineering Institute, Carnegie Mellon, Tech. Rep. January, 2009.

[19] US-CERT, "Combating the Insider Threat," National Cybersecurity and Communications Integration Center, no. May, pp. 61-64, 2014. [Online]. Available: https://www.us-cert.gov/sites/default/files/publications/ CombatingtheInsiderThreat_0.pdf

[20] K. Le, M. Brent Donnellan, S. K. Spilman, O. P. Garcia, and R. Conger, "Workers behaving badly: Associations between adolescent reports of the Big Five and counterproductive work behaviors in adulthood," Personality and Individual Differences, vol. 61-62, pp. 7-12, 2014. [Online]. Available: http://dx.doi.org/10.1016/j.paid.2013.12.016

[21] D. C. Blair, C. R. Barrett, C. W. Boustany Jr., S. Gorton, W. J. Lynn III, D. Wince-Smith, M. K. Young, and J. M. Huntsman Jr., "Update to the IP Commission Report on The Theft of American Intellectual Property: Reassessments of the challenge and United States Policy," U.S. Congress, Washington, D.C., Tech. Rep., 2017. 
[22] B. Casey, "The Impact of Intellectual Property Theft on the Economy," US Senate, Washington, D.C., Tech. Rep. August, 2012.

[23] Cybersecurity Insiders, "Insider Threat: 2018 Report," Crowd Research Partners, Tech. Rep., 2018. [Online]. Available: https://cdn2.hubspot.net// hubfs/5260286/PDFs/Whitepapers/insider-threat-report-2018-wp.pdf

[24] A. Cummings, T. Lewellen, D. Mcintire, A. P. Moore, and R. Trzeciak, "Insider threat study: Illicit cyber activity involving fraud in the u. s. financial services sector," Software Engineering Institute, Carnegie Mellon, Tech. Rep. July, 2012. [Online]. Available: https://resources.sei.cmu.edu/ asset_files/SpecialReport/2012_003_001_28137.pdf

[25] D. Cappelli, A. Moore, and R. Trzeciak, The CERT Guide to Insider Threats: How to Prevent, Detect, and respond to Information Technology Crimes (Theft, Sabotage, Fraud). Upper Saddle River, NJ: Addison-Wesley, 2012.

[26] A. P. Moore, D. M. Cappelli, T. C. Caron, E. Shaw, and R. F. Trzeciak, "Insider theft of intellectual property for business advantage: A preliminary model," CEUR Workshop Proceedings, vol. 469, pp. 1-21, 2009.

[27] J. Barling, K. E. Dupré, and E. K. Kelloway, "Predicting Workplace Aggression and Violence," Annual Review of Psychology, vol. 60, no. 1, pp. 671-692, 1 2009. [Online]. Available: http://www.annualreviews.org/doi/10. 1146/annurev.psych.60.110707.163629

[28] M. Cullen and P. R. Sackett, "Peronality and counterproductive work behavior," in Personality and Work, M. Barrick and A. Ryan, Eds. San Franciscio, CA: Jossey-Bass, 2003, pp. 150-182.

[29] M. S. Hershcovis, N. Turner, J. Barling, K. A. Arnold, K. E. Dupré, M. Inness, M. M. LeBlanc, and N. Sivanathan, "Predicting workplace aggression: A meta-analysis." Journal of Applied Psychology, vol. 92, no. 1, pp. 228-238, 2007. [Online]. Available: http://doi.apa.org/getdoi.cfm?doi=10. 1037/0021-9010.92.1.228

[30] M. Mount, R. Ilies, and E. Johnson, "Relationship of Personality Traits and Counterproductive Work Behaviors: The Mediating Effects of Job Satisfaction," Personnel Psychology, vol. 59, no. 3, pp. 591-622, 92006. [Online]. Available: http://doi.wiley.com/10.1111/j.1744-6570.2006.00048.x

[31] J. F. Salgado, "The Big Five Personality Dimensions and Counterproductive Behaviors," International Journal of Selection and Assessment, vol. 10, no. 1\&2, pp. 117-125, 3 2002. [Online]. Available: http://doi.wiley.com/10.1111/ $1468-2389.00198$

[32] J. Anglim, F. Lievens, L. Everton, S. L. Grant, and A. Marty, "HEXACO personality predicts counterproductive work behavior and organizational 
citizenship behavior in low-stakes and job applicant contexts," Journal of Research in Personality, vol. 77, pp. 11-20, 2018. [Online]. Available: https://doi.org/10.1016/j.jrp.2018.09.003

[33] P. R. Sackett and C. J. Devore, "Counterproductive Behaviors at Work," in Handbook of Industrial, Work and Organizational Psychology: Personnel Psychology handbook of industrial, work and organizational psychology: Personnel psychology. 1 Oliver's Yard, 55 City Road, London EC1Y 1SP United Kingdom: SAGE Publications Ltd, 2001, pp. 145-164. [Online]. Available: http://sk.sagepub.com/reference/hdbk_orgpsych1/n9.xml

[34] P. E. Spector and S. Fox, "The Stressor-Emotion Model of Counterproductive Work Behavior." in Counterproductive work behavior: Investigations of actors and targets., S. Fox and P. E. Spector, Eds. Washington: American Psychological Association, 2005, pp. 151-174. [Online]. Available: http://content.apa.org/books/10893-007

[35] I. N. A. M. F. Kozako, S. Z. Safin, and A. R. A. Rahim, "The Relationship of Big Five Personality Traits on Counterproductive Work Behaviour among Hotel Employees: An Exploratory Study," Procedia Economics and Finance, vol. 7, no. Icebr, pp. 181-187, 2013. [Online]. Available: http://dx.doi.org/10.1016/S2212-5671(13)00233-5

[36] C. M. Berry, D. S. Ones, and P. R. Sackett, "Interpersonal deviance, organizational deviance, and their common correlates: A review and metaanalysis." Journal of Applied Psychology, vol. 92, no. 2, pp. 410-424, 2007. [Online]. Available: http://doi.apa.org/getdoi.cfm?doi=10.1037/0021-9010.92. 2.410

[37] L. M. R. Bolton, L. K. Becker, and L. K. Barber, "Big Five trait predictors of differential counterproductive work behavior dimensions," Personality and Individual Differences, vol. 49, no. 5, pp. 537-541, 2010. [Online]. Available: http://dx.doi.org/10.1016/j.paid.2010.03.047

[38] J. M. Jensen and P. C. Patel, "Predicting counterproductive work behavior from the interaction of personality traits," Personality and Individual Differences, vol. 51, no. 4, pp. 466-471, 2011. [Online]. Available: http://dx.doi.org/10.1016/j.paid.2011.04.016

[39] S. Basu, Y. H. Victoria Chua, M. Wah Lee, W. G. Lim, T. Maszczyk, Z. Guo, and J. Dauwels, "Towards a data-driven behavioral approach to prediction of insider-threat," Proceedings - 2018 IEEE International Conference on Big Data, Big Data 2018, pp. 4994-5001, 2019.

[40] M. Maasberg, J. Warren, and N. L. Beebe, "The dark side of the insider: Detecting the insider threat through examination of dark triad personality traits," Proceedings of the Annual Hawaii International Conference on System Sciences, vol. 2015-March, pp. 3518-3526, 2015. 
[41] S. M. Ho, M. Kaarst-Brown, and I. Benbasat, "Trustworthiness attribution: Inquiry into insider threat detection," Journal of the Association for Information Science and Technology, vol. 69, no. 2, pp. 271-280, 2018.

[42] F. L. Greitzer, J. D. Lee, J. Purl, and A. K. Zaidi, "Design and Implementation of a Comprehensive Insider Threat Ontology," Procedia Computer Science, vol. 153, pp. 361-369, 2019. [Online]. Available: https://doi.org/10.1016/j.procs.2019.05.090

[43] G. Yang, L. Cai, A. Yu, J. Ma, D. Meng, and Y. Wu, "Potential Malicious Insiders Detection Based on a Comprehensive Security Psychological Model," Proceedings - IEEE 4th International Conference on Big Data Computing Service and Applications, BigDataService 2018, no. 2017, pp. 9-16, 2018.

[44] National Intelligence Council (U.S.), "Global trends : paradox of progress," U.S. Office of the Director of National Intelligence, Tech. Rep., 2017. [Online]. Available: https://www.dni.gov/index.php/global-trends-home

[45] M. Crenshaw, "The psychology of terrorism: An agenda for the 21st century," Political Psychology, vol. 21, no. 2, pp. 405-420, 2000.

[46] A. Abbasi and H. Chen, "Affect Intensity Analysis of Dark Web Forums," in 2007 IEEE Intelligence and Security Informatics. IEEE, 5 2007, pp. 282-288. [Online]. Available: http://ieeexplore.ieee.org/document/4258712/

[47] M. Petrovskiy and M. Chikunov, "Online extremism discovering through social network structure analysis," 2019 IEEE 2nd International Conference on Information and Computer Technologies, ICICT 2019, pp. 243-249, 2019.

[48] I. B. Arpinar, U. Kursuncu, and D. Achilov, "Social media analytics to identify and counter islamist extremism: Systematic detection, evaluation, and challenging of extremist narratives online," Proceedings - 2016 International Conference on Collaboration Technologies and Systems, CTS 2016, pp. 611-612, 2016.

[49] K. S. Douglas, C. D. Webster, S. D. Hart, and H. Belfrage, HCR-20V3: Assessing risk for violence - User guide. Burnaby, Canaga: Mental Health, Law, and Policy Institute, Simon Fraser University, 2013.

[50] R. Lara-Cabrera, A. Gonzalez-Pardo, M. Barhamgi, and D. Camacho, "Extracting radicalisation behavioural patterns from social network data," Proceedings International Workshop on Database and Expert Systems Applications, DEXA, vol. 2017-Augus, pp. 6-10, 2017.

[51] R. Lara-Cabrera, A. Gonzalez Pardo, K. Benouaret, N. Faci, D. Benslimane, and D. Camacho, "Measuring the Radicalisation Risk in Social Networks," IEEE Access, vol. 5, pp. 10892-10900, 2017. [Online]. Available: http: //ieeexplore.ieee.org/document/7935371/ 
[52] R. Lara-Cabrera, A. Gonzalez-Pardo, and D. Camacho, "Statistical analysis of risk assessment factors and metrics to evaluate radicalisation in Twitter," Future Generation Computer Systems, vol. 93, pp. 971-978, 2019. [Online]. Available: https://doi.org/10.1016/j.future.2017.10.046

[53] J. Torregrosa, I. Gilpérez-López, R. Lara-Cabrera, D. Garriga, and D. Camacho, "Can an automatic tool assess risk of radicalization online? A case study on Facebook," Proceedings - 2017 European Intelligence and Security Informatics Conference, EISIC 2017, vol. 2017-Janua, p. 165, 2017.

[54] I. Gilpérez-López, J. Torregrosa, M. Barhamgi, and D. Camacho, "An initial study on radicalization risk factors: Towards an assessment software tool," Proceedings - International Workshop on Database and Expert Systems Applications, DEXA, vol. 2017-Augus, pp. 11-16, 2017.

[55] M. Obaidi, R. Bergh, N. Akrami, and J. F. Dovidio, "The Personality of Extremists: Examining Violent and Non-Violent Defense of Muslims," PsyArxiv, 2020. [Online]. Available: https://psyarxiv.com/kry38/

[56] J. Ginges, S. Atran, S. Sachdeva, and D. Medin, "Psychology out of the laboratory: The challenge of violent extremism." American Psychologist, vol. 66, no. 6, pp. 507-519, 2011. [Online]. Available: http://doi.apa.org/getdoi.cfm?doi=10.1037/a0024715

[57] N. J. Smelser, Theory of collective behavior. New Orleans, LA: Quid Pro, 2011.

[58] A. Lankford, "Précis of The Myth of Martyrdom: What Really Drives Suicide Bombers, Rampage Shooters, and Other Self-Destructive Killers," Behavioral and Brain Sciences, vol. 37, no. 4, pp. 351362, 8 2014. [Online]. Available: https://www.cambridge.org/core/product/ identifier/S0140525X13001581/type/journal_article

[59] J. Victoroff, "The Mind of the Terrorist," Journal of Conflict Resolution, vol. 49, no. 1, pp. 3-42, 2 2005. [Online]. Available: http://journals.sagepub. com/doi/10.1177/0022002704272040

[60] M. D. Silber and A. Bhatt, "Radicalization in the west: The homegrown threat," The City of New York Police Department, NYPD Intelligence Division, New York, New York, USA, Tech. Rep., 2007. [Online]. Available: http://prtl-prd-web.nyc.gov/html/nypd/downloads/pdf/ public_information/NYPD_Report-Radicalization_in_the_West.pdf

[61] M. Sageman, Leaderless Jihad: Terror Networks in the Twenty-First Century. Philadelphia, PA: University of Pennsylvania Press, 2008.

[62] G. Morf, Le Terrorisme Quebecois. Montreal, Canada: Editions de l'Homme, 1970 . 
[63] M. Crenshaw, "The Causes of Terrorism," Comparative Politics, vol. 13, no. 4, p. 379, 1981. [Online]. Available: https://www.jstor.org/stable/421717

[64] L. Leone, A. Chirumbolo, and M. Desimoni, "The impact of the HEXACO personality model in predicting socio-political attitudes: The moderating role of interest in politics," Personality and Individual Differences, vol. 52, no. 3, pp. 416-421, 2012. [Online]. Available: http://dx.doi.org/10.1016/j.paid.2011.10.049

[65] M. Alizadeh, I. Weber, C. Cioffi-Revilla, S. Fortunato, and M. Macy, "Psychological and Personality Profiles of Political Extremists," (Preprint), 4 2017. [Online]. Available: http://arxiv.org/abs/1704.00119

[66] E. Bell, M. A. Woodley, J. A. Schermer, and P. A. Vernon, "Politics and the General Factor of Personality," Personality and Individual Differences, vol. 53, no. 5, pp. 546-551, 2012. [Online]. Available: http://dx.doi.org/10.1016/j.paid.2012.04.027

[67] S. Trip, M. I. Marian, A. Halmajan, M. I. Drugas, C. H. Bora, and G. Roseanu, "Irrational beliefs and personality traits as psychological mechanisms underlying the adolescents' extremist mind-set," Frontiers in Psychology, vol. 10, no. MAY, pp. 1-12, 2019.

[68] H. Chabrol, J. Bronchain, C. I. Morgades Bamba, and P. Raynal, "The Dark Tetrad and radicalization: personality profiles in young women," Behavioral Sciences of Terrorism and Political Aggression, vol. 12, no. 2, pp. 157-168, 4 2020. [Online]. Available: https: //www.tandfonline.com/doi/full/10.1080/19434472.2019.1646301

[69] M. Dewing, "Social Media: An Introduction," Library of Parliament, Canada, Ottowa, Canada, Tech. Rep., 20120. [Online]. Available: https://bdp.parl.ca/staticfiles/PublicWebsite/Home/ ResearchPublications/InBriefs/PDF/2010-03-e.pdf

[70] H. Allcott and M. Gentzkow, "Social media and fake news in the 2016 election," Journal of Economic Perspectives, vol. 31, no. 2, pp. 211-236, 2017.

[71] K. K. Kim, A. R. Lee, and U. K. Lee, "Impact of anonymity on roles of personal and group identities in online communities," Information and Management, vol. 56, no. 1, pp. 109-121, 2019. [Online]. Available: https://doi.org/10.1016/j.im.2018.07.005

[72] P. B. Lowry, J. Zhang, C. Wang, and M. Siponen, "Why do adults engage in cyberbullying on social media? An integration of online disinhibition and deindividuation effects with the social structure and social learning model," Information Systems Research, vol. 27, no. 4, pp. 962-986, 2016. 
[73] C.S.-W., "What doxxing is, and why it matters," 2014. [Online]. Available: https://www.economist.com/the-economist-explains/2014/03/10/ what-doxxing-is-and-why-it-matters

[74] Federal Bureau of Investivation, "Don't Make the Call: The New Phenomenon of 'Swatting'," 2008. [Online]. Available: https://archives.fbi.gov/archives/ news/stories/2008/february/swatting020408

[75] M. J. Moore, T. Nakano, A. Enomoto, and T. Suda, "Anonymity and roles associated with aggressive posts in an online forum," Computers in Human Behavior, vol. 28, no. 3, pp. 861-867, 2012. [Online]. Available: http://dx.doi.org/10.1016/j.chb.2011.12.005

[76] D. Bradbury, "Unveiling the dark web," Network Security, vol. 2014, no. 4, pp. 14-17, 2014. [Online]. Available: http://dx.doi.org/10.1016/S1353-4858(14) $70042-\mathrm{X}$

[77] J. S. Donath, "Identity Deception in the Virtual Community," Communities in Cyberspace, no. August 1996, pp. 29-59, 1999.

[78] N. Ellison, R. Heino, and J. Gibbs, "Managing Impressions Online: SelfPresentation Processes in the Online Dating Environment," Journal of Computer-Mediated Communication, vol. 11, no. 2, pp. 415-441, 2006.

[79] P. D. Ekstrom and C. M. Federico, "Personality and political preferences over time: Evidence from a multiwave longitudinal study," Journal of Personality, vol. 87, no. 2, pp. 398-412, 2019.

[80] N. Satherley, C. G. Sibley, and D. Osborne, "Identity, ideology, and personality: Examining moderators of affective polarization in New Zealand," Journal of Research in Personality, vol. 87, p. 103961, 2020. [Online]. Available: https://doi.org/10.1016/j.jrp.2020.103961

[81] F. Mosteller and D. Wallace, "Notes on an authorship problem," in Harvard Symposium on Digital Computers and their Applications, 1962, pp. 163-197.

[82] — - "Inference in an authorship problem: A comparative study of discrimination methods applied to the authorship of the disputed federalist papers," Journal of the American Statistical Association, vol. 58, no. 302, pp. 275-309, 1963.

[83] — Inference and disputed authorship: The Federalist. Center for the Study of Language and Inf., 1964.

[84] N. A. Novino, K. A. Sohn, and T. S. Chung, "A graph model based author attribution technique for single-class e-mail classification," 2015 IEEE/ACIS 14th International Conference on Computer and Information Science, ICIS 2015 Proceedings, pp. 191-196, 2015. 
[85] L. Fridman, S. Weber, R. Greenstadt, and M. Kam, "Active Authentication on Mobile Devices via Stylometry, Application Usage, Web Browsing, and GPS Location," IEEE Systems Journal, vol. 11, no. 2, pp. 513-521, 2017.

[86] J. Herz and A. Bellaachia, "The Authorship of Audacity: Data Mining and Stylometric Analysis of Barack Obama Speeches," Proceedings of the International Conference on Data Mining (DMIN), 2014.

[87] A. M. Kuruvilla and S. Varghese, "A Detection System to Counter Identity Deception in Social Media Applications," in 2015 International Conference on Circuit, Power, and Computer Technologies (ICCPCT). IEEE, 2015.

[88] R. Ragel, P. Herath, and U. Senanayake, "Authorship detection of SMS messages using unigrams," 2013 IEEE 8th International Conference on Industrial and Information Systems, ICIIS 2013 - Conference Proceedings, pp. 387-392, 2013.

[89] T. Neal, K. Sundararajan, A. Fatima, Y. Yan, Y. Xiang, and D. Woodard, "Surveying Stylometry Techniques and Applications," ACM Computing Surveys, vol. 50, no. 6, pp. 1-36, 11 2017. [Online]. Available: http: //dl.acm.org/citation.cfm?doid=3161158.3132039

[90] C. L. Huth, "The insider threat and employee privacy: An overview of recent case law," Computer Law and Security Review, vol. 29, no. 4, pp. 368-381, 2013. [Online]. Available: http://dx.doi.org/10.1016/j.clsr.2013.05.014

[91] Legal Information Institute, "Electronic Surveillance," 2017. [Online]. Available: https://www.law.cornell.edu/wex/electronic_surveillance

[92] "Executive Order 12333," 1981. [Online]. Available: https://www.archives. gov/federal-register/codification/executive-order/12333.html

[93] H. Chen, "Sentiment and affect analysis of Dark Web forums: Measuring radicalization on the internet," in 2008 IEEE International Conference on Intelligence and Security Informatics. IEEE, 6 2008, pp. 104-109. [Online]. Available: http://ieeexplore.ieee.org/document/4565038/

[94] G. Grefenstette, Y. Qu, D. A. Evans, and J. G. Shanahan, "Validating the coverage of lexical resources for affect analysis and automatically classifying new words along semantic axes," AAAI Spring Symposium - Technical Report, vol. SS-04-07, pp. 63-70, 2005.

[95] C. Ma, H. Prendinger, and M. Ishizuka, "Emotion estimation and reasoning based on affective textual interaction," Lecture Notes in Computer Science (including subseries Lecture Notes in Artificial Intelligence and Lecture Notes in Bioinformatics), vol. 3784 LNCS, pp. 622-628, 2005.

[96] J. Donath, K. Karahalios, and F. Viegas, "Visualizing conversation," Proceedings of the Hawaii International Conference on System Sciences, p. 74, 1999. 
[97] P. Subasic and A. Huettner, "Affect analysis of text using fuzzy semantic typing," IEEE Transactions on Fuzzy Systems, vol. 9, no. 4, pp. 483-496, 2001.

[98] Z. J. Chuang and C.-h. Wu, "Multi-modal emotion recognition from speech and text," Journal of Computational Linguistics and Chinese, vol. 9, no. 2, pp. 45-62, 2004. [Online]. Available: http://www.aclweb.org/anthology/O/O04/ O04-3004.pdf

[99] G. Mishne and M. De Rijke, "Capturing global mood levels using blog posts," AAAI Spring Symposium - Technical Report, vol. SS-06-03, no. August, pp. 145-152, 2006.

[100] G. Mishne, "Experiments with mood classification in blog posts," Proceedings of ACM SIGIR 2005 workshop on stylistic ..., 2005. [Online]. Available: http://citeseerx.ist.psu.edu/viewdoc/download?doi=10.1.1. $111.2693 \&$ rep $=$ rep1\&type $=$ pdf

[101] K. S. Sabra, R. N. Zantout, M. A. El Abed, and L. Hamandi, "Sentiment Analysis: Arabic sentiment lexicons," 2017 Sensors Networks Smart and Emerging Technologies, SENSET 2017, vol. 2017-Janua, pp. 1-4, 2017.

[102] A. Abbasi, Hsinchun Chen, S. Thoms, and Tianjun Fu, "Affect Analysis of Web Forums and Blogs Using Correlation Ensembles," IEEE Transactions on Knowledge and Data Engineering, vol. 20, no. 9, pp. 1168-1180, 92008. [Online]. Available: http://ieeexplore.ieee.org/document/4479460/

[103] W. JAMES, "II.-WHAT IS AN EMOTION ?" Mind, vol. os-IX, no. 34, pp. 188-205, 1884. [Online]. Available: https://academic.oup.com/mind/ article-lookup/doi/10.1093/mind/os-IX.34.188

[104] W. B. Cannon, "The James-Lange Theory of Emotions: A Critical Examination and an Alternative Theory," The American Journal of Psychology, vol. 100, no. 3/4, p. 567, 1987. [Online]. Available: https: //www.jstor.org/stable/1422695?origin=crossref

[105] S. Schachter and J. Singer, "Cognitive, social, and physiological determinants of emotional state." Psychological Review, vol. 69, no. 5, pp. 379-399, 1962. [Online]. Available: http://content.apa.org/journals/rev/69/5/379

[106] M. B. Arnold, Emotion and personality. New York, New York, USA: Columbia University Press, 1960.

[107] N. H. Frijda, The emotions. Cambridge: Cambridge University Press, 1986.

[108] R. S. Lazarus, Psychological Stress and the Coping Process. New York, New York, USA: McGraw-Hill, 1966. 
[109] I. J. Roseman and C. A. Smith, "Appraisal Theory: Overview, Assumptions, Varieties, controversies," in Appraisal processes in emotion: Theory, methods, research, K. R. Scherer, A. Schorr, and T. Johnstone, Eds. London: London University Press, 2001, pp. 3-19.

[110] R. Plutchik, "A general psychoevolutionary theory of emotion," in Theories of Emotion, R. Plutchik and H. Kellerman, Eds. New York, New York, USA: Academic Press, 1980, pp. 3-33.

[111] _ _ "Emotions: a general psychoevolutionary theory," in Approaches to Emotions, K. R. Scherer and P. Ekman, Eds. Hillsdale, NJ: Lawrence Erlbaum Associates, 1984, pp. 197-219.

[112] P. Ekman, "An Argument for Basic Emotions," Cognition and Emotion, vol. 6, no. 3, pp. 169-200, 1992. [Online]. Available: http://www.paulekman.com/wp-content/uploads/ 2009/02/Universality-Of-Emotional-Expression-A-personal-History.pdfhttp: //doi.apa.org/getdoi.cfm?doi=10.1037/0033-295X.99.3.550

[113] _ - "Basic Emotions," in Handbook of Cognition and Emotions, T. Dalgleish and M. J. Power, Eds. Chichester, UK: John Wiley \& Sons, Ltd, 1999, ch. 3, pp. 45-60. [Online]. Available: https://www.paulekman.com/wp-content/uploads/ 2013/07/Basic-Emotions.pdfhttp://doi.wiley.com/10.1002/0470013494

[114] — "Universals and Cultural Differences in Facial Expressions of Emotion," in Nebraska Symposium on Motivation, J. Cole, Ed. Lincoln University of Nebraska Press, 1972, vol. 19, pp. 207-282. [Online]. Available: papers3://publication/uuid/FDC5E29A-0E28-4DDF-B1A4-F53FEE0B4F70

[115] — _ "Are There Basic Emotions?" pp. 550-553, 1992.

[116] Machine Elf 1735, "File:Plutchik-wheel.svg," 2011. [Online]. Available: https://commons.wikimedia.org/wiki/File:Plutchik-wheel.svg

[117] ChaoticBrain, "File:Plutchik Dyads.svg," 2019. [Online]. Available: https: //commons.wikimedia.org/wiki/File:Plutchik_Dyads.svg

[118] C. Strapparava and R. Mihalcea, "SemEval-2007 task 14: Affective text," $A C L$ 2007 - SemEval 2007 - Proceedings of the 4 th International Workshop on Semantic Evaluations, no. June, pp. 70-74, 2007.

[119] S. Mohammad, F. Bravo-Marquez, M. Salameh, and S. Kiritchenko, "SemEval2018 Task 1: Affect in Tweets," Proceedings of the 12th International Workshop on Semantic Evaluation (SemEval-2018), pp. 1-17, 2018.

[120] The MITRE Corporation, "Technical Report and Annotation Instructions: Language Processing for Virtual Communications: Egyptian Arabic," The MITRE Corporation, Tech. Rep., 2017. 
[121] G. J. Boyle, "Myers-Briggs Type Indicator (MBTI): Some Psychometric Limitations," Australian Psychologist, vol. 30, no. 1, pp. 71-74, 31995. [Online]. Available: http://doi.wiley.com/10.1111/j.1742-9544.1995.tb01750.x

[122] S. R. WALlaCE, W. V. CLARKE, and R. J. DRY, "The Activity Vector Analysis as a Selector of Life Insurance Salesmen," Personnel Psychology, vol. 9, no. 3, pp. 337-345, 9 1956. [Online]. Available: http://doi.wiley.com/10.1111/j.1744-6570.1956.tb01072.x

[123] W. M. Marston, Emotions of Normal People. London: Kegan Paul, Trench, Trubner \& Co, 1928. [Online]. Available: https://archive.org/details/ emotionsofnormal032195mbp/page/n7/mode/2up

[124] W. J. Camara, J. S. Nathan, and A. E. Puente, "Psychological test usage: Implications in professional psychology." Professional Psychology: Research and Practice, vol. 31, no. 2, pp. 141-154, 4 2000. [Online]. Available: http://doi.apa.org/getdoi.cfm?doi=10.1037/0735-7028.31.2.141

[125] B. C. Schiele, A. B. Baker, and S. R. Hathaway, "The Minnesota Multiphasic Peronality Inventory," Journal-lancet, no. 63, pp. 292-297, 1943.

[126] Y. S. Ben-Porath and D. L. Davis, Case Studies for Interpreting the MMPI-A. Minneapolis, MN: University of Minnesota Press, 1996.

[127] Y. S. Ben-Porath, Interpreting the MMPI-2-RF. Minneapolis, MN: University of Minnesota Press, 2012.

[128] J. N. Butcher and C. L. Williams, "Personality Assessment with the MMPI-2: Historical Roots, International Adaptations, and Current Challenges," Applied Psychology: Health and Well-Being, vol. 1, no. 1, pp. 105-135, 3 2009. [Online]. Available: http://doi.wiley.com/10.1111/j.1758-0854.2008.01007.x

[129] R. B. Cattell, Use of Factor Analysis in Behavioral and Life Sciences. New York, New York, USA: Plenum, 1978.

[130] E. C. Tupes and R. E. Christal, "Recurrent Personality Factors Based on Trait Ratings," USAF ASD Technical Report, vol. 60, no. 61-97, pp. 225-251, 1961. [Online]. Available: http://doi.wiley.com/10.1111/j.1467-6494.1992.tb00973.x

[131] L. R. Goldberg, "From Ace to Zombie: Same explorations in the language of personality," in Advances in Personality Assessment, J. N. Butcher, Ed. Hillsdale, NJ: Erlbaum, 1982, pp. 201-234.

[132] R. R. McCrae and P. T. Costa, "Validation of the five-factor model of personality across instruments and observers." Journal of Personality and Social Psychology, vol. 52, no. 1, pp. 81-90, 1987. [Online]. Available: http://doi.apa.org/getdoi.cfm?doi=10.1037/0022-3514.52.1.81 
[133] B. S. Connelly, D. S. Ones, and O. S. Chernyshenko, "Introducing the Special Section on Openness to Experience: Review of Openness Taxonomies, Measurement, and Nomological Net," Journal of Personality Assessment, vol. 96, no. 1, pp. 1-16, 1 2014. [Online]. Available: http://www.tandfonline.com/doi/abs/10.1080/00223891.2013.830620

[134] R. R. McCrae and O. P. John, "An Introduction to the Five-Factor Model and Its Applications," Journal of Personality, vol. 60, no. 2, pp. 175-215, 1992.

[135] R. Hogan, Hogan Personality Inventory Manual. Minneapolis, MN: National Computer Systems, 1986.

[136] D. J. Ozer and V. Benet-Martínez, "Personality and the Prediction of Consequential Outcomes," Annual Review of Psychology, vol. 57, no. 1, pp. 401-421, 1 2006. [Online]. Available: http://www.annualreviews.org/doi/10. 1146/annurev.psych.57.102904.190127

[137] D. Watson and L. A. Clark, "Extraversion and Its Positive Emotional Core," in Handbook of Personality Psychology. Elsevier, 1997, pp. 767-793. [Online]. Available: https://linkinghub.elsevier.com/retrieve/pii/ B9780121346454500305

[138] S. Rothmann and E. P. Coetzer, "The big five personality dimensions and job performance," SA Journal of Industrial Psychology, vol. 29, no. 1, 102003. [Online]. Available: http://sajip.co.za/index.php/sajip/article/view/88

[139] W. G. Graziano and N. Eisenberg, "Agreeableness: A Dimension of Personality," in Handbook of Personality Psychology. Elsevier, 1997, pp. 795-824. [Online]. Available: https://linkinghub.elsevier.com/retrieve/pii/ B9780121346454500317

[140] A. B. Zonderman, S. V. Stone, and P. T. Costa, "Age and neuroticism as risk factors for the incidence of diagnoses of psychotic and neurotic disorders." in Annual Convention of the American Psychological Association, New Orleans, LA, 1989.

[141] M. C. Ashton, K. Lee, M. Perugini, P. Szarota, R. E. de Vries, L. Di Blas, K. Boies, and B. De Raad, "A Six-Factor Structure of Personality-Descriptive Adjectives: Solutions From Psycholexical Studies in Seven Languages." Journal of Personality and Social Psychology, vol. 86, no. 2, pp. 356-366, 2004. [Online]. Available: http://doi.apa.org/getdoi.cfm?doi=10.1037/0022-3514.86.2.356

[142] M. C. Ashton and K. Lee, "Empirical, theoretical, and practical advantages of the HEXACO model of personality structure," Personality and Social Psychology Review, vol. 11, no. 2, pp. 150-166, 2007.

[143] M. Kosinski, S. C. Matz, S. D. Gosling, V. Popov, and D. Stillwell, "Facebook as a research tool for the social sciences: Opportunities, challenges, ethical 
considerations, and practical guidelines," American Psychologist, vol. 70, no. 6, pp. 543-556, 2015.

[144] N. Shuyo, "Language Detection Library for Java," 2010. [Online]. Available: https://github.com/shuyo/language-detection

[145] S. Day, J. Brown, Z. Thomas, I. Gregory, L. Bass, and G. Dozier, "Adversarial authorship, AuthorWebs, and entropy-based evolutionary clustering," 2016 25th International Conference on Computer Communications and Networks, ICCCN 2016, no. August, 2016.

[146] C. Faust, G. Dozier, J. Xu, and M. C. King, "Adversarial authorship, interactive evolutionary hill-climbing, and author CAAT-III," in 2017 IEEE Symposium Series on Computational Intelligence (SSCI). IEEE, 11 2017, pp. 1-8. [Online]. Available: http://ieeexplore.ieee.org/document/8285355/

[147] J. Gaston, M. Narayanan, G. Dozier, D. L. Cothran, C. Arms-Chavez, M. Rossi, M. C. King, and J. Xu, "Authorship Attribution via Evolutionary Hybridization of Sentiment Analysis, LIWC, and Topic Modeling Features," Proceedings of the 2018 IEEE Symposium Series on Computational Intelligence, SSCI 2018, no. September 2019, pp. 933-940, 2019.

[148] B. Onyshkevych, "Broad Operational Language Translation (BOLT) (Archived)." [Online]. Available: https://www.darpa.mil/program/ broad-operational-language-translation

[149] J. Tracey, H. Lee, S. Strassel, and S. Ismael, "BOLT Arabic Discussion Forums LDC2018T10," Philadelphia, 2018. [Online]. Available: https: //catalog.ldc.upenn.edu/LDC2018T10

[150] J. Tracey, H. Lee, S. Strassel, and S. Chen, "BOLT Chinese Discussion Forums LDC2016T05," Philadelphia, 2016. [Online]. Available: https: //catalog.ldc.upenn.edu/LDC2016T05

[151] G. A. Miller, "WordNet: A Lexical Database for English," Communications of the ACM, vol. 38, no. 11, pp. 39-41, 1995.

[152] C. Fellbaum, WordNet: An Electronic Lexical Database. Cambridge, MA: MIT Press, 1998.

[153] L. Bloomfield, Language. London: George Allen \& Unwin, 1935.

[154] J. Coleman, Life of Slang. Oxford: Oxford University Press, 2012.

[155] C. Manning, M. Surdeanu, J. Bauer, J. Finkel, S. Bethard, and D. McClosky, "The Stanford CoreNLP Natural Language Processing Toolkit," in Proceedings of the 52nd Annual Meeting of the Association for Computational Linguistics: System Demonstrations, 2014, pp. 55-60. 
[156] A. Pasha, M. Al-Badrashiny, M. Diab, A. El Kholy, R. Eskander, N. Habash, M. Pooleery, O. Rambow, and R. M. Roth, "MADAMIRA: A fast, comprehensive tool for morphological analysis and disambiguation of Arabic," Proceedings of the 9th International Conference on Language Resources and Evaluation, LREC 2014, pp. 1094-1101, 2014.

[157] D. Graff, M. Maamouri, B. Bouziri, S. Krouna, S. Kulick, and T. Buckwalter, "Standard Arabic Morphological Analyzer (SAMA) Version 3.1," 2009. [Online]. Available: https://catalog.ldc.upenn.edu/LDC2010L01

[158] P. Rodrigues, V. Novak, C. Anton Rytting, J. Yelle, and J. Boutz, "Arabic data science toolkit: An API for Arabic language feature extraction," LREC 2018 - 11th International Conference on Language Resources and Evaluation, pp. 1239-1245, 2019.

[159] Y. R. Tausczik and J. W. Pennebaker, "The psychological meaning of words: LIWC and computerized text analysis methods," Journal of Language and Social Psychology, vol. 29, no. 1, pp. 24-54, 2010.

[160] W. Youyou, M. Kosinski, and D. Stillwell, "Computer-based personality judgments are more accurate than those made by humans," Proceedings of the $\mathrm{Na}$ tional Academy of Sciences of the United States of America, vol. 112, no. 4, pp. 1036-1040, 2015.

[161] S. Argamon, S. Dhawle, M. Koppel, and J. W. Pennebaker, "Lexical Predictors of Personality Type," in 2005 Joint Annual Meeting of the Interface and the Classification Society of North America, 2005.

[162] F. Mairesse, M. A. Walker, M. R. Mehl, and R. K. Moore, "Using linguistic cues for the automatic recognition of personality in conversation and text," Journal of Artificial Intelligence Research, vol. 30, pp. 457-500, 2007.

[163] M. Wilson, "MRC psycholinguistic database: Machine-usable dictionary, version 2.00," Behavior Research Methods, Instruments, $\&$ Computers, vol. 20, no. 1, pp. 6-10, 1988.

[164] J. Oberlander and S. Nowson, "Whose thumb is it anyway? Classifying author personaltiy from weblog text," in Proceedings of the 44th Annual Meeting of the Association for Computational Linguistics (ACL), no. July, 2006, pp. 627-634.

[165] S. Nowson and J. Oberlander, "Identifying more bloggers: Towards large scale personality classification of personal weblogs," ICWSM 2007 - International Conference on Weblogs and Social Media, 2007.

[166] J. Golbeck, C. Robles, and K. Turner, "Predicting personality with social media," in Proceedings of the 2011 annual conference extended abstracts on Human factors in computing systems - CHI EA '11. New York, New York, USA: ACM Press, 2011, p. 253. [Online]. Available: http://portal.acm.org/citation.cfm?doid=1979742.1979614 
[167] J. Golbeck, C. Robles, M. Edmondson, and K. Turner, "Predicting personality from twitter," Proceedings - 2011 IEEE International Conference on Privacy, Security, Risk and Trust and IEEE International Conference on Social Computing, PASSAT/SocialCom 2011, pp. 149-156, 2011.

[168] D. Quercia, M. Kosinski, D. Stillwell, and J. Crowcroft, "Our twitter profiles, our selves: Predicting personality with twitter," in IEEE Third International Conference on Privacy, Security, Risk and Trust and IEEE Third International Conference on Social Computing, 2011, pp. 180-185.

[169] F. Celli, F. Pianesi, D. Stillwell, and M. Kosinski, "Workshop on computational personality recognition: Shared task," AAAI Workshop - Technical Report, vol. WS-13-01, pp. 2-5, 2013.

[170] G. Farnadi, G. Sitaraman, S. Sushmita, F. Celli, M. Kosinski, D. Stillwell, S. Davalos, M. F. Moens, and M. De Cock, "Computational personality recognition in social media," User Modeling and User-Adapted Interaction, vol. 26, no. 2-3, pp. 109-142, 2016.

[171] G. Park, H. A. Schwartz, J. C. Eichstaedt, M. L. Kern, M. Kosinski, D. J. Stillwell, L. H. Ungar, and M. E. P. Seligman, "Automatic personality assessment through social media language." Journal of Personality and Social Psychology, vol. 108, no. 6, pp. 934-952, 6 2015. [Online]. Available: http://dx.doi.org/10.1037/pspp0000020\%5Cnhttp://dx.doi.org/10.1037/ pspp0000020.supphttp://doi.apa.org/getdoi.cfm?doi=10.1037/pspp0000020

[172] H. A. Schwartz, J. C. Eichstaedt, M. L. Kern, L. Dziurzynski, S. M. Ramones, M. Agrawal, A. Shah, M. Kosinski, D. Stillwell, M. E. Seligman, and L. H. Ungar, "Personality, Gender, and Age in the Language of Social Media: The Open-Vocabulary Approach," PLoS ONE, vol. 8, no. 9, 2013.

[173] D. M. Blei, A. Y. Ng, and M. I. Jordan, "Latent Dirichlet Allocation," Journal of Machine Learning Research, vol. 3, pp. 993-1022, 2003.

[174] K. Sundararajan, T. Neal, Y. Yan, A. Fatima, Y. Xiang, K. Reese, and D. Woodard, "Performance and Feature Analysis for Large-Scale Authorship Attribution," Journal for IC Research \& Development (submitted), 2017.

[175] M. Potthast, S. Braun, T. Buz, F. Duffhauss, F. Friederich, J. M. Gulzow, J. Kohler, W. Lotzsch, F. Muller, M. E. Muller, R. Passmann, B. Reinke, L. Rettenmeier, T. Romestsch, T. Sommer, M. Trager, S. Wilhelm, B. Stein, E. Stamatatos, and M. Hagen, "Who Wrote the Web? Revisiting Influential Author Identification Research Applicable to Information Retrieval," in Advances in Information Retrieval, ser. Lecture Notes in Computer Science, N. Ferro, F. Crestani, M.-F. Moens, J. Mothe, F. Silvestri, G. M. Di Nunzio, C. Hauff, and G. Silvello, Eds. Cham: Springer International Publishing, 2016, vol. 9626, pp. 393-407. [Online]. Available: http://link.springer.com/10.1007/978-3-319-30671-1 
[176] V. Keselj, F. Peng, N. Cercone, and C. Thomas, "N-Gram-based Author Profiles for Authorship Attribution," Pacific Association for Computational Linguistics, 2003.

[177] M. Koppel, J. Schler, and S. Argamon, "Authorship attribution in the wild," Language Resources and Evaluation, vol. 45, no. 1, pp. 83-94, 32011. [Online]. Available: http://link.springer.com/10.1007/s10579-009-9111-2https: //link.aps.org/doi/10.1103/PhysRevLett.88.048702

[178] E. Stamatatos, "Author Identification Using Imbalanced and Limited Training Texts," in 18th International Conference on Database and Expert Systems Applications (DEXA 2007). IEEE, 9 2007, pp. 237-241. [Online]. Available: http://ieeexplore.ieee.org/document/4312893/

[179] W. J. Teahan and D. J. Harper, "Using Compression-Based Language Models for Text Categorization," in Language Modeling for Information Retrieval. Dordrecht: Springer Netherlands, 2003, pp. 141-165. [Online]. Available: http://link.springer.com/10.1007/978-94-017-0171-6_7

[180] W. R. Bennett, Scientific and engineering problem-solving with the computer. Englewood Cliffs, New Jersey: Prentice-Hall, 1976.

[181] D. Benedetto, E. Caglioti, and V. Loreto, "Language Trees and Zipping," Physical Review Letters, vol. 88, no. 4, p. 048702, 1 2002. [Online]. Available: http: //arxiv.org/abs/cond-mat/0108530http://dx.doi.org/10.1103/PhysRevLett. 88.048702https://link.aps.org/doi/10.1103/PhysRevLett.88.048702

[182] M. Koppel, J. Schler, and E. Bonchek-Dokow, "Measuring Differentiability: Unmasking Pseudonymous Authors," Journal of Machine Learning Research, vol. 8, no. Jun, pp. 1261-1276, 2007. [Online]. Available: http: //www.jmlr.org/papers/v8/koppel07a.html

[183] K. Sundararajan and D. L. Woodard, "What constitutes "style" in authorship attribution?" 27th International Conference on Computational Linguistics, pp. 2814-2822, 2018.

[184] T. Neal, K. Sundararajan, and D. Woodard, "Exploiting Linguistic Style as a Cognitive Biometric for Continuous Verification," in 2018 International Conference on Biometrics (ICB). IEEE, 2 2018, pp. 270-276. [Online]. Available: https://ieeexplore.ieee.org/document/8411232/

[185] Y. Wu, M. Schuster, Z. Chen, Q. V. Le, M. Norouzi, W. Macherey, M. Krikun, Y. Cao, Q. Gao, K. Macherey, J. Klingner, A. Shah, M. Johnson, X. Liu, L. Kaiser, S. Gouws, Y. Kato, T. Kudo, H. Kazawa, K. Stevens, G. Kurian, N. Patil, W. Wang, C. Young, J. Smith, J. Riesa, A. Rudnick, O. Vinyals, G. Corrado, M. Hughes, and J. Dean, "Google's Neural Machine Translation System: Bridging the Gap between Human and Machine Translation," CoRR, 9 2016. [Online]. Available: http://arxiv.org/abs/1609.08144 
[186] M. N. Al-Kabi, T. M. Hailat, E. M. Al-Shawakfa, and I. M. Alsmadi, "Evaluating English to Arabic Machine Translation Using BLEU," in International Journal of Advanced Computer Science and Applications, vol. 4, no. 1, 2013. [Online]. Available: www.ijacsa.thesai.org

[187] K. Papineni, S. Roukos, T. Ward, and W.-J. Zhu, "BLEU: A Method for Automatic Evaluation of Machine Translation," in Proceedings of the 40th Annual Meeting of the Association for Computational Linguistics (ACL), Philadelphia, PA, 2002, pp. 311-318. [Online]. Available: https: //www.aclweb.org/anthology/P02-1040.pdf

[188] P. Burman, "A comparative study of ordinary cross-validation, v-fold crossvalidation and the repeated learning-testing methods," Biometrika, vol. 76, no. 3, pp. 503-514, 1989.

[189] T. Hastie, R. Tibshirani, and J. Friedman, The Elements of Statistical Learning: Data Mining, Inference, and Prediction, 2nd ed. New York, New York, USA: Springer, 2011.

[190] P. Zhang, "Model Selection via Multifold Cross Validation," Annals of Statistics, vol. 21, no. 1, pp. 299-313, 1993. [Online]. Available: https://projecteuclid.org/download/pdf_1/euclid.aos/1176349027

[191] Q. S. Xu and Y. Z. Liang, "Monte Carlo cross validation," Chemometrics and Intelligent Laboratory Systems, vol. 56, no. 1, pp. 1-11, 2001.

[192] H. B. Mann and D. R. Whitney, "On a Test of Whether one of Two Random Variables is Stochastically Larger than the Other," The Annals of Mathematical Statistics, vol. 18, no. 1, pp. 50-60, 3 1947. [Online]. Available: http://projecteuclid.org/euclid.aoms/1177730491

[193] M. P. Fay and M. A. Proschan, "Wilcoxon-Mann-Whitney or t-test? On assumptions for hypothesis tests and multiple interpretations of decision rules," Statistics Surveys, vol. 4, pp. 1-39, 2010. [Online]. Available: http://projecteuclid.org/euclid.ssu/1266847666

[194] J. Ruscio, "A probability-based measure of effect size: Robustness to base rates and other factors." Psychological Methods, vol. 13, no. 1, pp. 19-30, 2008. [Online]. Available: http://doi.apa.org/getdoi.cfm?doi=10.1037/1082-989X.13. 1.19 


\section{APPENDIX A: FEATURE SIGNIFICANCE TABLE}

Table A1. Personality features were tested to determine if translations had a significant impact on the features used to estimate personality traits in the algorithm. In total, 141 of the features Accepted the null hypothesis (they were not statistically significantly different). The remaining 1,870 rejected the null hypothesis indicating that translations had an impact on those 1,870 features.

\begin{tabular}{|c|c|c|c|c|c|}
\hline Feature & EN median & FL median & u-value & p-value & $\mathbf{H}_{\mathbf{0}}$ \\
\hline topic0 & $3.4723 \mathrm{e}-05$ & $2.9037 \mathrm{e}-05$ & $2.32522 \mathrm{e}+06$ & $6.8712 \mathrm{e}-12$ & Reject \\
\hline topic1 & 0.00017084 & 0.00014516 & $2.33841 \mathrm{e}+06$ & $5.0089 \mathrm{e}-11$ & Reject \\
\hline topic2 & $9.9062 \mathrm{e}-05$ & $5.3927 \mathrm{e}-05$ & $1.60226 \mathrm{e}+06$ & $3.5321 \mathrm{e}-116$ & Reject \\
\hline topic3 & 0.0001228 & $4.5225 \mathrm{e}-05$ & $1.2822 \mathrm{e}+06$ & $1.867 \mathrm{e}-197$ & Reject \\
\hline topic4 & 0.0001521 & $8.7758 \mathrm{e}-05$ & $1.83851 \mathrm{e}+06$ & $9.701 \mathrm{e}-70$ & Reject \\
\hline topic5 & 0.00017083 & 0.0001161 & $1.98804 \mathrm{e}+06$ & $1.9628 \mathrm{e}-46$ & Reject \\
\hline topic6 & $7.955 \mathrm{e}-05$ & 2.3991e-05 & $1.58556 \mathrm{e}+06$ & $4.1755 \mathrm{e}-120$ & Reject \\
\hline topic7 & 0.00011667 & $3.7927 \mathrm{e}-05$ & $1.33048 \mathrm{e}+06$ & $1.7698 \mathrm{e}-184$ & Reject \\
\hline topic8 & 0.00013431 & $9.4224 \mathrm{e}-05$ & $2.20309 \mathrm{e}+06$ & $1.3158 \mathrm{e}-20$ & Reject \\
\hline topic9 & 0.00010734 & $5.0461 \mathrm{e}-05$ & $1.88832 \mathrm{e}+06$ & $9.4281 \mathrm{e}-61$ & Reject \\
\hline topic10 & $4.2269 \mathrm{e}-05$ & $2.583 \mathrm{e}-05$ & $2.18252 \mathrm{e}+06$ & $3.2475 \mathrm{e}-23$ & Reject \\
\hline topic11 & 0.00013196 & $7.8082 \mathrm{e}-05$ & $1.83702 \mathrm{e}+06$ & $7.785 \mathrm{e}-70$ & Reject \\
\hline topic12 & $2.2291 \mathrm{e}-05$ & $9.005 \mathrm{e}-06$ & $1.76914 \mathrm{e}+06$ & $5.6476 \mathrm{e}-82$ & Reject \\
\hline topic13 & 0.00012994 & $7.9005 \mathrm{e}-05$ & $1.7571 \mathrm{e}+06$ & $6.882 \mathrm{e}-84$ & Reject \\
\hline topic14 & 0.0001007 & 0.00011062 & $2.58663 \mathrm{e}+06$ & 0.18914 & Accept \\
\hline topic15 & 0.00015581 & 0.00011087 & $2.02953 \mathrm{e}+06$ & $1.8441 \mathrm{e}-40$ & Reject \\
\hline topic16 & 0.00011785 & $5.5671 \mathrm{e}-05$ & $1.32806 \mathrm{e}+06$ & $9.0062 \mathrm{e}-185$ & Reject \\
\hline topic17 & 0.00010016 & $9.7456 \mathrm{e}-05$ & $2.52681 \mathrm{e}+06$ & 0.013353 & Reject \\
\hline topic18 & 0.00017933 & 0.00012495 & $1.90933 \mathrm{e}+06$ & $3.6783 \mathrm{e}-58$ & Reject \\
\hline topic19 & 0.000132 & $9.4173 \mathrm{e}-05$ & $2.03634 \mathrm{e}+06$ & $4.2564 \mathrm{e}-40$ & Reject \\
\hline topic20 & $9.3253 \mathrm{e}-05$ & $7.0657 \mathrm{e}-05$ & $2.17258 \mathrm{e}+06$ & $1.1269 \mathrm{e}-24$ & Reject \\
\hline topic21 & 0.00011291 & $7.4583 \mathrm{e}-05$ & $2.12176 \mathrm{e}+06$ & $1.1088 \mathrm{e}-29$ & Reject \\
\hline topic22 & $6.1965 \mathrm{e}-05$ & $8.568 \mathrm{e}-06$ & 993255 & $3.6174 \mathrm{e}-291$ & Reject \\
\hline topic23 & 0.00013168 & $9.1414 \mathrm{e}-05$ & $1.89857 \mathrm{e}+06$ & $1.4562 \mathrm{e}-59$ & Reject \\
\hline topic24 & 0.00011575 & 0.00010644 & $2.37961 \mathrm{e}+06$ & $1.2539 \mathrm{e}-08$ & Reject \\
\hline topic25 & $2.5295 \mathrm{e}-05$ & $1.1833 \mathrm{e}-05$ & $1.56563 \mathrm{e}+06$ & $5.5042 \mathrm{e}-123$ & Reject \\
\hline
\end{tabular}


Table A1 - Continued from previous page

\begin{tabular}{|c|c|c|c|c|c|}
\hline Feature & EN median & FL median & u-value & p-value & $\mathbf{H}_{0}$ \\
\hline topic26 & 0.00013506 & 0.00012425 & $2.35307 \mathrm{e}+06$ & $3.541 \mathrm{e}-10$ & Reject \\
\hline topic27 & $1.5173 \mathrm{e}-05$ & $4.3376 \mathrm{e}-05$ & $1.81909 \mathrm{e}+06$ & $1.5092 \mathrm{e}-71$ & Reject \\
\hline topic28 & $8.5455 \mathrm{e}-05$ & $7.5134 \mathrm{e}-05$ & $2.42072 \mathrm{e}+06$ & $2.8534 \mathrm{e}-06$ & Reject \\
\hline topic29 & $1.1959 \mathrm{e}-05$ & $2.3282 \mathrm{e}-05$ & $2.06166 \mathrm{e}+06$ & $7.6006 \mathrm{e}-36$ & Reject \\
\hline topic30 & 0.00011504 & $4.8327 \mathrm{e}-05$ & $1.51046 \mathrm{e}+06$ & $6.5558 \mathrm{e}-137$ & Reject \\
\hline topic31 & $6.3176 \mathrm{e}-05$ & $5.085 \mathrm{e}-05$ & $2.0597 \mathrm{e}+06$ & $2.8118 \mathrm{e}-37$ & Reject \\
\hline topic32 & $4.9245 \mathrm{e}-05$ & $3.4181 \mathrm{e}-05$ & $1.83492 \mathrm{e}+06$ & $1.6214 \mathrm{e}-70$ & Reject \\
\hline topic33 & $7.0588 \mathrm{e}-05$ & $7.8216 \mathrm{e}-05$ & $2.55991 \mathrm{e}+06$ & 0.076794 & Accept \\
\hline topi & 0.00010055 & $7.2569 \mathrm{e}-05$ & $2.19083 \mathrm{e}+06$ & $1.3607 \mathrm{e}-22$ & Reject \\
\hline topic35 & 0.00012157 & $8.316 \mathrm{e}-05$ & $2.05699 \mathrm{e}+06$ & $2.3063 \mathrm{e}-37$ & Reject \\
\hline topic36 & 0.00014085 & 0.0001232 & $2.18818 \mathrm{e}+06$ & $4.7848 \mathrm{e}-23$ & Reject \\
\hline topic37 & $8.2977 \mathrm{e}-05$ & $3.4252 \mathrm{e}-05$ & $1.81734 \mathrm{e}+06$ & $1.9978 \mathrm{e}-73$ & Reject \\
\hline topic38 & $3.3733 \mathrm{e}-05$ & $3.7172 \mathrm{e}-05$ & $2.52716 \mathrm{e}+06$ & 0.017495 & Reject \\
\hline topic39 & 867 & e- 05 & $2.03443 \mathrm{e}+06$ & $1.0297 \mathrm{e}-39$ & Reject \\
\hline & 4826 & & $2.18262 \mathrm{e}+06$ & 23 & \\
\hline topic41 & $1.6333 \mathrm{e}-05$ & $4.9401 \mathrm{e}-05$ & $1.59318 \mathrm{e}+06$ & $2.0475 \mathrm{e}-107$ & Reject \\
\hline topic42 & 0.0001557 & 0.00015573 & $2.58001 \mathrm{e}+06$ & 0.14617 & Accept \\
\hline topic43 & $4.459 \mathrm{e}-05$ & $3.5758 \mathrm{e}-05$ & $2.29424 \mathrm{e}+06$ & $3.8387 \mathrm{e}-14$ & Reject \\
\hline topic44 & 0.00013897 & 1702 & $2.21992 \mathrm{e}+06$ & $4.1647 \mathrm{e}-20$ & Reject \\
\hline & & & $2.03071 \mathrm{e}+06$ & & \\
\hline topic46 & 0.00012187 & $7.7275 \mathrm{e}-05$ & $2.03189 \mathrm{e}+06$ & $1.1343 \mathrm{e}-40$ & Reject \\
\hline topic47 & $2 \mathrm{e}-05$ & $1 \mathrm{e}-06$ & $1.76358 \mathrm{e}+06$ & $7.6292 \mathrm{e}-83$ & Reject \\
\hline topic48 & 0.00011235 & $9 \mathrm{e}-05$ & $2.36648 \mathrm{e}+06$ & $6.1234 \mathrm{e}-09$ & Reject \\
\hline topic49 & e- 05 & $9.7752 \mathrm{e}-05$ & $2.09357 \mathrm{e}+06$ & $4.2161 \mathrm{e}-33$ & Reject \\
\hline topic50 & 0.00 & 0.00010388 & $2.16531 \mathrm{e}+06$ & $2.6756 \mathrm{e}-25$ & Reject \\
\hline topic51 & $9 \mathrm{e}-05$ & $6 \mathrm{e}-05$ & 956151 & $4.1023 \mathrm{e}-300$ & Reject \\
\hline topic52 & $1.0375 \mathrm{e}-05$ & $1.8286 \mathrm{e}-05$ & $2.10604 \mathrm{e}+06$ & $3.4693 \mathrm{e}-16$ & Reject \\
\hline & 0.00013377 & $3 e-05$ & $1.81816 \mathrm{e}+06$ & $2.8782 \mathrm{e}-73$ & Reject \\
\hline topic54 & $46 \mathrm{e}-05$ & $6 e-05$ & $2.50224 \mathrm{e}+06$ & 0.0026373 & Reject \\
\hline & -05 & 05 & $2.04387 \mathrm{e}+06$ & 2.9123e-39 & Reject \\
\hline topic56 & 0.00014608 & 0.00010516 & $2.08850 \mathrm{e}+06$ & $1.4565 \mathrm{e}-33$ & Reject \\
\hline topic57 & $8.9475 \mathrm{e}-05$ & $6.467 \mathrm{e}-05$ & $2.15276 \mathrm{e}+06$ & $2.9216 \mathrm{e}-26$ & Reject \\
\hline & $2.475 \mathrm{e}-05$ & $6.9883 \mathrm{e}-05$ & $1.63202 \mathrm{e}+06$ & 3.8613e-103 & Reject \\
\hline top & 0.00012192 & 0.00014649 & $2.40530 \mathrm{e}+06$ & $2.867 \mathrm{e}-07$ & Reject \\
\hline topic60 & 0.00016811 & 0.0001895 & $2.36916 \mathrm{e}+06$ & $3.205 \mathrm{e}-09$ & Reject \\
\hline & 0.00017849 & 0.00011179 & $2.00585 \mathrm{e}+06$ & $3.2849 \mathrm{e}-43$ & Reject \\
\hline topic62 & 0.00016293 & 0.00018767 & $2.42398 \mathrm{e}+06$ & $3.2355 \mathrm{e}-06$ & Reject \\
\hline topic63 & 0.0001333 & 0.00010114 & $2.15325 \mathrm{e}+06$ & $1.5668 \mathrm{e}-26$ & Reject \\
\hline & $2.9494 \mathrm{e}-05$ & $1.5315 \mathrm{e}-05$ & $1.72692 \mathrm{e}+06$ & $5.2988 \mathrm{e}-90$ & Reject \\
\hline topic65 & 0.00010102 & $7.4709 \mathrm{e}-05$ & $1.95592 \mathrm{e}+06$ & $2.8376 \mathrm{e}-51$ & Reject \\
\hline & 0.00015426 & 0.00012229 & $2.32757 \mathrm{e}+06$ & $2.2627 \mathrm{e}-11$ & Reject \\
\hline topic67 & $6.2202 \mathrm{e}-05$ & $2.922 \mathrm{e}-05$ & $2.05226 \mathrm{e}+06$ & $5.791 \mathrm{e}-38$ & Reject \\
\hline
\end{tabular}


Table A1 - Continued from previous page

\begin{tabular}{|c|c|c|c|c|c|}
\hline Feature & EN median & FL median & u-value & p-value & $\mathbf{H}_{0}$ \\
\hline topic68 & 0.00018674 & $1.4795 \mathrm{e}-05$ & $1.58884 \mathrm{e}+06$ & $5.2741 \mathrm{e}-119$ & Reject \\
\hline topic69 & 0.00021944 & 0.00021067 & $2.48408 \mathrm{e}+06$ & 0.00058938 & Reject \\
\hline topic70 & 0.00013265 & $9.9467 \mathrm{e}-05$ & $1.89826 \mathrm{e}+06$ & $6.6957 \mathrm{e}-60$ & Reject \\
\hline topic71 & 0.00010101 & 0.0001273 & $2.2164 \mathrm{e}+06$ & $2.015 \mathrm{e}-20$ & Reject \\
\hline topic72 & $4.4463 \mathrm{e}-05$ & $1.5545 \mathrm{e}-05$ & $1.22424 \mathrm{e}+06$ & $7.2164 \mathrm{e}-215$ & Reject \\
\hline topic73 & 0.00010664 & $9.1728 \mathrm{e}-05$ & $2.3687 \mathrm{e}+06$ & $3.9964 \mathrm{e}-09$ & Reject \\
\hline topic74 & $6.0936 \mathrm{e}-05$ & $6.2628 \mathrm{e}-05$ & $2.54912 \mathrm{e}+06$ & 0.040566 & Reject \\
\hline topic75 & 0.00014489 & $9.5295 \mathrm{e}-05$ & $1.93678 \mathrm{e}+06$ & $5.7943 \mathrm{e}-54$ & Reject \\
\hline topic76 & $1.2605 \mathrm{e}-06$ & $1.3695 \mathrm{e}-06$ & $2.51975 \mathrm{e}+06$ & 0.010852 & Reject \\
\hline topic77 & 0.00013411 & $8.4171 \mathrm{e}-05$ & $1.69384 \mathrm{e}+06$ & 2.0769e-96 & Reject \\
\hline topic78 & $5.5942 \mathrm{e}-05$ & $4.0437 \mathrm{e}-05$ & $1.8527 \mathrm{e}+06$ & $1.7165 \mathrm{e}-67$ & Reject \\
\hline topic79 & 0.00012624 & $8.5989 \mathrm{e}-05$ & $2.00275 \mathrm{e}+06$ & $1.4471 \mathrm{e}-42$ & Reject \\
\hline topic80 & 0.00010953 & $6.6959 \mathrm{e}-05$ & $1.80619 \mathrm{e}+06$ & $4.3672 \mathrm{e}-75$ & Reject \\
\hline topic81 & 0.00011271 & 0.00010578 & $2.51095 \mathrm{e}+06$ & 0.0043776 & Reject \\
\hline topic82 & 0.0001564 & 0.00018839 & $2.27217 \mathrm{e}+06$ & $9.8093 \mathrm{e}-16$ & Reject \\
\hline topic83 & $2.9445 \mathrm{e}-05$ & $1.5755 \mathrm{e}-05$ & $2.0572 \mathrm{e}+06$ & $1.0255 \mathrm{e}-36$ & Reject \\
\hline topic84 & 0.0001301 & 0.00016004 & $2.21566 \mathrm{e}+06$ & $1.3861 \mathrm{e}-20$ & Reject \\
\hline topic85 & 0.00013555 & 0.00012589 & $2.45132 \mathrm{e}+06$ & $3.5308 \mathrm{e}-05$ & Reject \\
\hline topic86 & 0.00012728 & $9.2444 \mathrm{e}-05$ & $1.98380 \mathrm{e}+06$ & $9.7434 \mathrm{e}-47$ & Reject \\
\hline topic87 & $6.8162 \mathrm{e}-05$ & $6.4495 \mathrm{e}-05$ & $2.50706 \mathrm{e}+06$ & 0.0039483 & Reject \\
\hline topic88 & 0.00012525 & $7.2791 \mathrm{e}-05$ & $1.71739 \mathrm{e}+06$ & $2.1551 \mathrm{e}-91$ & Reject \\
\hline topic89 & 0.0001062 & $1.1345 \mathrm{e}-05$ & 775855 & 0 & Reject \\
\hline topic90 & 0.00015547 & 0.00019079 & $2.23373 \mathrm{e}+06$ & $5.4945 \mathrm{e}-19$ & Reject \\
\hline topic91 & $9.3534 \mathrm{e}-05$ & 0.00012449 & $2.17169 \mathrm{e}+06$ & $1.1653 \mathrm{e}-24$ & Reject \\
\hline topic92 & $6.8368 \mathrm{e}-05$ & $1.0705 \mathrm{e}-05$ & 999699 & $7.4865 \mathrm{e}-285$ & Reject \\
\hline topic93 & $3.9405 \mathrm{e}-05$ & $2.5394 \mathrm{e}-05$ & $2.13532 \mathrm{e}+06$ & $2.0168 \mathrm{e}-28$ & Reject \\
\hline topic94 & $7.7104 \mathrm{e}-05$ & $4.7369 \mathrm{e}-05$ & $1.55485 \mathrm{e}+06$ & $9.0476 \mathrm{e}-127$ & Reject \\
\hline topic95 & $9.192 \mathrm{e}-05$ & $6.3074 \mathrm{e}-05$ & $1.89564 \mathrm{e}+06$ & $2.5747 \mathrm{e}-60$ & Reject \\
\hline topic96 & $3.3086 \mathrm{e}-05$ & $1.9049 \mathrm{e}-05$ & $1.97527 \mathrm{e}+06$ & $4.3754 \mathrm{e}-48$ & Reject \\
\hline topic97 & 0.00014788 & 0.00012186 & $2.12434 \mathrm{e}+06$ & $5.8477 \mathrm{e}-29$ & Reject \\
\hline topic98 & $8.815 \mathrm{e}-05$ & 7.9517e-05 & $2.45237 \mathrm{e}+06$ & $4.8076 \mathrm{e}-05$ & Reject \\
\hline topic99 & 0.00014017 & 0.00011367 & $2.05833 \mathrm{e}+06$ & $1.9033 \mathrm{e}-37$ & Reject \\
\hline topic100 & $5.9221 \mathrm{e}-05$ & $4.0836 \mathrm{e}-05$ & $2.15389 \mathrm{e}+06$ & $2.2833 \mathrm{e}-26$ & Reject \\
\hline topic101 & 0.00012433 & $8.9841 \mathrm{e}-05$ & $2.15953 \mathrm{e}+06$ & $3.639 \mathrm{e}-25$ & Reject \\
\hline topic102 & $8.3935 \mathrm{e}-05$ & 0.00032635 & $1.4122 \mathrm{e}+06$ & $9.1558 \mathrm{e}-159$ & Reject \\
\hline topic103 & 0.00014681 & 0.00010939 & $2.07448 \mathrm{e}+06$ & $5.3729 \mathrm{e}-35$ & Reject \\
\hline topic104 & 0.00013607 & 0.00017841 & $2.21048 \mathrm{e}+06$ & $1.3658 \mathrm{e}-20$ & Reject \\
\hline topic105 & 0.00010995 & 0.00010048 & $2.53939 \mathrm{e}+06$ & 0.029698 & Reject \\
\hline topic106 & $1.8722 \mathrm{e}-05$ & $1.4427 \mathrm{e}-05$ & $2.19514 \mathrm{e}+06$ & $2.1575 \mathrm{e}-22$ & Reject \\
\hline topic107 & $8.7836 \mathrm{e}-05$ & $6.7303 \mathrm{e}-05$ & $2.19059 \mathrm{e}+06$ & $1.0038 \mathrm{e}-22$ & Reject \\
\hline topic108 & 7.323e-05 & $5.4748 \mathrm{e}-05$ & $2.06124 \mathrm{e}+06$ & $5.8151 \mathrm{e}-37$ & Reject \\
\hline topic109 & $3.5925 \mathrm{e}-08$ & $2.4595 \mathrm{e}-06$ & 369056 & $6.079 \mathrm{e}-52$ & Reject \\
\hline
\end{tabular}


Table A1 - Continued from previous page

\begin{tabular}{|c|c|c|c|c|c|}
\hline Feature & EN median & FL median & u-value & p-value & $\mathrm{H}_{0}$ \\
\hline topic110 & $8.2209 \mathrm{e}-05$ & $4.8885 \mathrm{e}-05$ & $1.93505 \mathrm{e}+06$ & $3.1803 \mathrm{e}-54$ & Reject \\
\hline topic111 & 0.00012561 & 0.00011831 & $2.47754 \mathrm{e}+06$ & 0.00038398 & Reject \\
\hline topic112 & 0.00016978 & 0.00015631 & $2.41262 \mathrm{e}+06$ & $6.5975 \mathrm{e}-07$ & Reject \\
\hline topic113 & 0.00012804 & $8.8228 \mathrm{e}-05$ & $1.98597 \mathrm{e}+06$ & $2.7465 \mathrm{e}-43$ & Reject \\
\hline topic114 & $8.6921 \mathrm{e}-05$ & $9.3108 \mathrm{e}-05$ & $2.51975 \mathrm{e}+06$ & 0.0071665 & Reject \\
\hline topic115 & $9.7732 \mathrm{e}-05$ & $7.8165 \mathrm{e}-05$ & $2.15093 \mathrm{e}+06$ & $8.9865 \mathrm{e}-27$ & Reject \\
\hline topic116 & $5.8407 \mathrm{e}-05$ & $9.3373 \mathrm{e}-07$ & 973495 & $1.3641 \mathrm{e}-289$ & Reject \\
\hline topic117 & 0.00016956 & 0.00012287 & $2.11433 \mathrm{e}+06$ & $1.3124 \mathrm{e}-30$ & Reject \\
\hline topic118 & $7.8983 \mathrm{e}-05$ & $5.6494 \mathrm{e}-05$ & $2.01615 \mathrm{e}+06$ & $1.7899 \mathrm{e}-42$ & Reject \\
\hline topic119 & $5.5432 \mathrm{e}-05$ & $3.9737 \mathrm{e}-05$ & $2.22031 \mathrm{e}+06$ & $6.9729 \mathrm{e}-20$ & Reject \\
\hline topic120 & 0.00013976 & 0.00010139 & $1.8068 \mathrm{e}+06$ & $4.1321 \mathrm{e}-75$ & Reject \\
\hline topic121 & 0.0001491 & 0.00013732 & $2.43704 \mathrm{e}+06$ & 8.833e-06 & Reject \\
\hline topic122 & $9.2863 \mathrm{e}-05$ & $9.0642 \mathrm{e}-05$ & $2.59167 \mathrm{e}+06$ & 0.22898 & Accept \\
\hline topic123 & 0.000 & $3 e-05$ & $1.97697 \mathrm{e}+06$ & $1.967 \mathrm{e}-47$ & Reject \\
\hline top & 0.00019261 & & $2.0645 \mathrm{e}+06$ & $1.8905 \mathrm{e}-36$ & \\
\hline topic125 & 0.00014716 & $8.7657 \mathrm{e}-05$ & $1.99081 \mathrm{e}+06$ & $8.6068 \mathrm{e}-46$ & Reject \\
\hline topic126 & 0.00013678 & 0.00014562 & $2.47163 \mathrm{e}+06$ & 0.00021462 & Reject \\
\hline topic127 & 0.0001176 & $9.6031 \mathrm{e}-05$ & $2.07462 \mathrm{e}+06$ & $4.2243 \mathrm{e}-35$ & Reject \\
\hline topic128 & 0.00014393 & $7.7164 \mathrm{e}-05$ & $1.61514 \mathrm{e}+06$ & $4.6664 \mathrm{e}-112$ & Reject \\
\hline topic129 & 0.000 & 0.000224 & $1.43736 \mathrm{e}+06$ & 156 & \\
\hline topic130 & 0.00011232 & 0.00010191 & $2.55056 \mathrm{e}+06$ & 0.043439 & Reject \\
\hline topic131 & 0.00012016 & 0.00010835 & $2.39326 \mathrm{e}+06$ & $7.8809 \mathrm{e}-08$ & Reject \\
\hline topic132 & $9.8222 \mathrm{e}-05$ & $1 \mathrm{e}-05$ & $2.58864 \mathrm{e}+06$ & 0.18759 & Accept \\
\hline topic133 & $5.1108 \mathrm{e}-05$ & $7.0088 \mathrm{e}-06$ & 979371 & $4.6639 \mathrm{e}-294$ & Reject \\
\hline topic134 & $9.3321 \mathrm{e}-05$ & $4.3711 \mathrm{e}-05$ & $1.81434 \mathrm{e}+06$ & $8.5985 \mathrm{e}-74$ & Reject \\
\hline topic135 & 0.00014815 & $5 e-05$ & $1.48171 \mathrm{e}+06$ & $2.464 \mathrm{e}-142$ & Reject \\
\hline topic136 & 0.00013264 & $9.1084 \mathrm{e}-05$ & $2.15628 \mathrm{e}+06$ & $5.1427 \mathrm{e}-26$ & Reject \\
\hline & $5.8038 \mathrm{e}-05$ & $2.7227 \mathrm{e}-05$ & $2.02289 \mathrm{e}+06$ & $7.5832 \mathrm{e}-42$ & Reject \\
\hline topic138 & 0.00014145 & 0.00015831 & $2.39452 \mathrm{e}+06$ & $8.0174 \mathrm{e}-08$ & Reject \\
\hline topic139 & 0.00012936 & $7.7971 \mathrm{e}-05$ & $1.63853 \mathrm{e}+06$ & $4.2351 \mathrm{e}-108$ & Reject \\
\hline 140 & $8.3502 \mathrm{e}-05$ & $5.3442 \mathrm{e}-05$ & $1.95426 \mathrm{e}+06$ & $5.6289 \mathrm{e}-51$ & Reject \\
\hline topic141 & $4.3341 \mathrm{e}-05$ & $6.7753 \mathrm{e}-05$ & $1.81861 \mathrm{e}+06$ & $2.3764 \mathrm{e}-73$ & Reject \\
\hline 142 & 0.00019794 & 0.0001697 & $2.30739 \mathrm{e}+06$ & $3.4225 \mathrm{e}-13$ & Reject \\
\hline 143 & 0.00010873 & $7.158 \mathrm{e}-05$ & $1.85360 \mathrm{e}+06$ & $3.5046 \mathrm{e}-67$ & Reject \\
\hline topic144 & $6.9961 \mathrm{e}-05$ & $6.0761 \mathrm{e}-05$ & $2.4693 \mathrm{e}+06$ & 0.00019419 & Reject \\
\hline top & 0.0002174 & 0.0003016 & $1.82776 \mathrm{e}+06$ & $1.319 \mathrm{e}-71$ & Reject \\
\hline topic146 & 0.00011261 & $6.7127 \mathrm{e}-05$ & $1.97198 \mathrm{e}+06$ & $8.0967 \mathrm{e}-49$ & Reject \\
\hline topic147 & $6.3286 \mathrm{e}-05$ & $1.6514 \mathrm{e}-06$ & 894187 & 0 & Reject \\
\hline topic148 & $6.3383 \mathrm{e}-05$ & $3.9049 \mathrm{e}-06$ & 710068 & 0 & Reject \\
\hline topic149 & $7.4537 \mathrm{e}-05$ & $3.7064 \mathrm{e}-05$ & $1.65284 \mathrm{e}+06$ & $1.5866 \mathrm{e}-104$ & Reject \\
\hline topic150 & $9.7895 \mathrm{e}-05$ & $6.7104 \mathrm{e}-05$ & $2.2026 \mathrm{e}+06$ & $1.3802 \mathrm{e}-21$ & Reject \\
\hline topic151 & $3.0901 \mathrm{e}-05$ & $6.3344 \mathrm{e}-06$ & $1.22388 \mathrm{e}+06$ & $2.2098 \mathrm{e}-212$ & Reject \\
\hline
\end{tabular}


Table A1 - Continued from previous page

\begin{tabular}{|c|c|c|c|c|c|}
\hline Feature & EN median & FL median & u-value & p-value & $\mathbf{H}_{0}$ \\
\hline topic152 & $4.8606 \mathrm{e}-05$ & $1.9832 \mathrm{e}-05$ & $1.61111 \mathrm{e}+06$ & $3.2025 \mathrm{e}-114$ & Reject \\
\hline topic153 & 0.00013576 & $8.7081 \mathrm{e}-05$ & $2.08448 \mathrm{e}+06$ & $1.8883 \mathrm{e}-33$ & Reject \\
\hline topic154 & 0.00012415 & 0.00013075 & $2.53427 \mathrm{e}+06$ & 0.020213 & Reject \\
\hline topic155 & $6.0564 \mathrm{e}-05$ & $4.5116 \mathrm{e}-05$ & $2.0047 \mathrm{e}+06$ & $3.8663 \mathrm{e}-44$ & Reject \\
\hline topic156 & 0.00014031 & $9.3187 \mathrm{e}-05$ & $1.91171 \mathrm{e}+06$ & $1.2151 \mathrm{e}-57$ & Reject \\
\hline topic157 & 0.00015173 & 0.00012024 & $2.05281 \mathrm{e}+06$ & $3.9115 \mathrm{e}-38$ & Reject \\
\hline topic158 & 0.0001093 & $9.0851 \mathrm{e}-05$ & $2.32681 \mathrm{e}+06$ & $7.4101 \mathrm{e}-12$ & Reject \\
\hline topic159 & 0.00016015 & 0.00010257 & $1.73203 \mathrm{e}+06$ & $5.0335 \mathrm{e}-89$ & Reject \\
\hline topic160 & 0.00012132 & $9.1075 \mathrm{e}-05$ & $2.18317 \mathrm{e}+06$ & $4.7344 \mathrm{e}-23$ & Reject \\
\hline topic161 & 0.00012841 & 0.00011539 & $2.29881 \mathrm{e}+06$ & $8.2834 \mathrm{e}-14$ & Reject \\
\hline topic162 & 0.00013564 & 0.00012954 & $2.55785 \mathrm{e}+06$ & 0.054847 & Accept \\
\hline topic163 & 0.00015866 & $7.4065 \mathrm{e}-05$ & $1.4013 \mathrm{e}+06$ & $1.5327 \mathrm{e}-164$ & Reject \\
\hline topic164 & 0.00010746 & $9.6977 \mathrm{e}-05$ & $2.40953 \mathrm{e}+06$ & $5.2299 \mathrm{e}-07$ & Reject \\
\hline topic165 & $7.5267 \mathrm{e}-05$ & $4.7586 \mathrm{e}-05$ & $2.17202 \mathrm{e}+06$ & $1.2575 \mathrm{e}-24$ & Reject \\
\hline topic166 & $4.9863 \mathrm{e}-05$ & $5.2534 \mathrm{e}-05$ & $2.62426 \mathrm{e}+06$ & 0.47374 & Accept \\
\hline topic167 & 0.00010155 & 0.00011697 & $2.43567 \mathrm{e}+06$ & $8.6208 \mathrm{e}-06$ & Reject \\
\hline topic168 & $3.4169 \mathrm{e}-05$ & $2.378 \mathrm{e}-05$ & $2.08210 \mathrm{e}+06$ & $3.3807 \mathrm{e}-34$ & Reject \\
\hline topic169 & 0.00013573 & $7.4175 \mathrm{e}-05$ & $1.70667 \mathrm{e}+06$ & $1.1261 \mathrm{e}-93$ & Reject \\
\hline topic170 & $7.3288 \mathrm{e}-05$ & 7.3473e-05 & $2.56872 \mathrm{e}+06$ & 0.09159 & Accept \\
\hline topic171 & 0.00012121 & $9.3157 \mathrm{e}-05$ & $2.16849 \mathrm{e}+06$ & $5.5895 \mathrm{e}-25$ & Reject \\
\hline topic172 & 0.00011874 & $5.9432 \mathrm{e}-05$ & $1.68156 \mathrm{e}+06$ & $1.985 \mathrm{e}-99$ & Reject \\
\hline topic173 & 0.00013834 & 0.00010469 & $1.98853 \mathrm{e}+06$ & $2.2947 \mathrm{e}-46$ & Reject \\
\hline topic174 & 0.00014038 & 0.00010804 & $2.25556 \mathrm{e}+06$ & $8.4451 \mathrm{e}-17$ & Reject \\
\hline topic175 & 0.00010318 & $6.2311 \mathrm{e}-05$ & $1.98115 \mathrm{e}+06$ & $2.1421 \mathrm{e}-47$ & Reject \\
\hline topic176 & 0.0001212 & $9.1654 \mathrm{e}-05$ & $1.99237 \mathrm{e}+06$ & $5.8556 \mathrm{e}-46$ & Reject \\
\hline topic177 & 0.00013347 & 0.00010511 & $2.18758 \mathrm{e}+06$ & $6.6637 \mathrm{e}-23$ & Reject \\
\hline topic178 & $7.9712 \mathrm{e}-05$ & $6.5014 \mathrm{e}-05$ & $2.26631 \mathrm{e}+06$ & $2.7887 \mathrm{e}-16$ & Reject \\
\hline topic179 & $9.4473 \mathrm{e}-05$ & 0.0001215 & $2.22404 \mathrm{e}+06$ & $7.803 \mathrm{e}-20$ & Reject \\
\hline topic180 & $9.1151 \mathrm{e}-05$ & $9.0206 \mathrm{e}-05$ & $2.55134 \mathrm{e}+06$ & 0.040516 & Reject \\
\hline topic181 & $7.3412 \mathrm{e}-05$ & $6.0206 \mathrm{e}-05$ & $2.22936 \mathrm{e}+06$ & $3.4482 \mathrm{e}-19$ & Reject \\
\hline topic182 & 0.00014185 & $9.2458 \mathrm{e}-05$ & $2.05873 \mathrm{e}+06$ & $2.133 \mathrm{e}-37$ & Reject \\
\hline topic183 & $7.0501 \mathrm{e}-05$ & $5.4531 \mathrm{e}-05$ & $2.18662 \mathrm{e}+06$ & $3.3147 \mathrm{e}-23$ & Reject \\
\hline topic184 & $5.1584 \mathrm{e}-05$ & $2.8584 \mathrm{e}-05$ & $1.57893 \mathrm{e}+06$ & $1.9643 \mathrm{e}-121$ & Reject \\
\hline topic185 & 0.00010149 & $9.7866 \mathrm{e}-05$ & $2.58226 \mathrm{e}+06$ & 0.15745 & Accept \\
\hline topic186 & 0.00014194 & 0.00012134 & $2.23841 \mathrm{e}+06$ & $1.7126 \mathrm{e}-18$ & Reject \\
\hline topic187 & 0.0001294 & $8.8477 \mathrm{e}-05$ & $2.1148 \mathrm{e}+06$ & $8.7385 \mathrm{e}-31$ & Reject \\
\hline topic188 & $4.3839 \mathrm{e}-05$ & $2.9864 \mathrm{e}-05$ & $1.93863 \mathrm{e}+06$ & $1.0642 \mathrm{e}-53$ & Reject \\
\hline topic189 & $6.8592 \mathrm{e}-05$ & $4.882 \mathrm{e}-05$ & $2.11768 \mathrm{e}+06$ & $3.0263 \mathrm{e}-30$ & Reject \\
\hline topic190 & 0.00012619 & $8.1922 \mathrm{e}-05$ & $2.07048 \mathrm{e}+06$ & $7.8264 \mathrm{e}-36$ & Reject \\
\hline topic191 & 0.0001157 & $4.1367 \mathrm{e}-05$ & $1.50548 \mathrm{e}+06$ & $6.7731 \mathrm{e}-138$ & Reject \\
\hline topic192 & 0.00021556 & 0.00019889 & $2.36898 \mathrm{e}+06$ & $3.1316 \mathrm{e}-09$ & Reject \\
\hline topic193 & 0.00012856 & 0.00010749 & $2.24107 \mathrm{e}+06$ & $3.551 \mathrm{e}-18$ & Reject \\
\hline
\end{tabular}


Table A1 - Continued from previous page

\begin{tabular}{|c|c|c|c|c|c|}
\hline Feature & EN median & FL median & u-value & p-value & $\mathbf{H}_{0}$ \\
\hline topic194 & 0.00018155 & 0.00010738 & $1.88637 \mathrm{e}+06$ & 8.6666e-61 & Reject \\
\hline topic195 & $6.4504 \mathrm{e}-05$ & $3.7002 \mathrm{e}-05$ & $1.8878 \mathrm{e}+06$ & $3.1391 \mathrm{e}-60$ & Reject \\
\hline topic196 & $6.0633 \mathrm{e}-05$ & $2.491 \mathrm{e}-05$ & $1.50313 \mathrm{e}+06$ & $1.7414 \mathrm{e}-138$ & Reject \\
\hline topic197 & 0.00012831 & 0.00011628 & $2.51409 \mathrm{e}+06$ & 0.0094807 & Reject \\
\hline topic198 & 0.00010227 & $4.9035 \mathrm{e}-05$ & $1.66054 \mathrm{e}+06$ & $3.7972 \mathrm{e}-99$ & Reject \\
\hline topic199 & $5.4469 \mathrm{e}-05$ & $1.1358 \mathrm{e}-05$ & 776602 & 0 & Reject \\
\hline topic200 & $9.0705 \mathrm{e}-05$ & $1.0595 \mathrm{e}-05$ & $1.02389 \mathrm{e}+06$ & $1.7273 \mathrm{e}-278$ & Reject \\
\hline topic201 & 0.00013342 & 0.00011365 & $2.22911 \mathrm{e}+06$ & 2.1778e-19 & Reject \\
\hline topic202 & $3.6609 \mathrm{e}-05$ & $4.1113 \mathrm{e}-05$ & $2.51389 \mathrm{e}+06$ & 0.0061778 & Reject \\
\hline topic203 & $3.7197 \mathrm{e}-05$ & $3.5956 \mathrm{e}-05$ & $2.57216 \mathrm{e}+06$ & 0.12483 & Accept \\
\hline topic204 & $6.8096 \mathrm{e}-05$ & $4.653 \mathrm{e}-05$ & $2.12541 \mathrm{e}+06$ & $1.6979 \mathrm{e}-29$ & Reject \\
\hline topic205 & 0.00010018 & $5.6224 \mathrm{e}-05$ & $1.81604 \mathrm{e}+06$ & $1.7133 \mathrm{e}-73$ & Reject \\
\hline topic206 & $9.5243 \mathrm{e}-05$ & $5.272 \mathrm{e}-05$ & $1.46872 \mathrm{e}+06$ & $9.1255 \mathrm{e}-148$ & Reject \\
\hline topic207 & $3.9606 \mathrm{e}-05$ & $2.7502 \mathrm{e}-05$ & $2.0861 \mathrm{e}+06$ & $7.4236 \mathrm{e}-34$ & Reject \\
\hline topic208 & $6.0614 \mathrm{e}-05$ & $5.7341 \mathrm{e}-05$ & $2.49745 \mathrm{e}+06$ & 0.0016095 & Reject \\
\hline topic209 & 0.00013285 & 0.0001569 & $2.49855 \mathrm{e}+06$ & 0.0018912 & Reject \\
\hline topic210 & 0.00014747 & 0.00014155 & $2.57226 \mathrm{e}+06$ & 0.10527 & Accept \\
\hline topic211 & $8.9838 \mathrm{e}-05$ & $8.1421 \mathrm{e}-05$ & $2.50921 \mathrm{e}+06$ & 0.010479 & Reject \\
\hline topic212 & $4.8421 \mathrm{e}-05$ & $3.1802 \mathrm{e}-05$ & $2.08159 \mathrm{e}+06$ & $2.1725 \mathrm{e}-34$ & Reject \\
\hline topic213 & $9.2774 \mathrm{e}-06$ & $5.5262 \mathrm{e}-06$ & $2.19377 \mathrm{e}+06$ & $1.6315 \mathrm{e}-22$ & Reject \\
\hline topic214 & $8.8465 \mathrm{e}-05$ & 0.00020847 & $1.89671 \mathrm{e}+06$ & $4.2122 \mathrm{e}-52$ & Reject \\
\hline topic215 & 0.00015971 & 7.3597e-05 & $1.30106 \mathrm{e}+06$ & $2.1466 \mathrm{e}-192$ & Reject \\
\hline topic216 & $3.96 \mathrm{e}-05$ & $3.4675 \mathrm{e}-05$ & $2.44212 \mathrm{e}+06$ & $2.769 \mathrm{e}-05$ & Reject \\
\hline topic217 & 0.00011511 & $7.5568 \mathrm{e}-05$ & $1.53966 \mathrm{e}+06$ & $1.6962 \mathrm{e}-130$ & Reject \\
\hline topic218 & $2.613 \mathrm{e}-05$ & $4.922 \mathrm{e}-05$ & $1.87735 \mathrm{e}+06$ & $2.5491 \mathrm{e}-58$ & Reject \\
\hline topic219 & 0.00017897 & 0.00018457 & $2.57512 \mathrm{e}+06$ & 0.11224 & Accept \\
\hline topic220 & 0.00015962 & 0.00010637 & $1.95667 \mathrm{e}+06$ & $9.6948 \mathrm{e}-51$ & Reject \\
\hline topic221 & $4.0753 \mathrm{e}-05$ & $2.2389 \mathrm{e}-05$ & $1.59861 \mathrm{e}+06$ & $1.9364 \mathrm{e}-116$ & Reject \\
\hline topic222 & 0.00011339 & $9.0132 \mathrm{e}-05$ & $2.14659 \mathrm{e}+06$ & $3.0985 \mathrm{e}-27$ & Reject \\
\hline topic223 & $7.284 \mathrm{e}-05$ & $5.9766 \mathrm{e}-05$ & $2.27532 \mathrm{e}+06$ & $1.4225 \mathrm{e}-15$ & Reject \\
\hline topic224 & $3.8741 \mathrm{e}-05$ & $8.5645 \mathrm{e}-07$ & 959916 & $3.5521 \mathrm{e}-302$ & Reject \\
\hline topic225 & 0.00010842 & 0.00010369 & $2.62252 \mathrm{e}+06$ & 0.44772 & Accept \\
\hline topic226 & 0.00013986 & $9.5426 \mathrm{e}-05$ & $1.88178 \mathrm{e}+06$ & $2.1307 \mathrm{e}-62$ & Reject \\
\hline topic227 & 0.00011157 & $8.211 \mathrm{e}-05$ & $2.29907 \mathrm{e}+06$ & $8.653 \mathrm{e}-14$ & Reject \\
\hline topic228 & 0.00010947 & $8.6152 \mathrm{e}-05$ & $2.28471 \mathrm{e}+06$ & $3.8661 \mathrm{e}-14$ & Reject \\
\hline topic229 & $4.7196 \mathrm{e}-05$ & $4.2719 \mathrm{e}-06$ & $1.31501 \mathrm{e}+06$ & $2.4541 \mathrm{e}-186$ & Reject \\
\hline topic230 & 0.00014233 & 0.00012505 & $2.31768 \mathrm{e}+06$ & $2.1215 \mathrm{e}-12$ & Reject \\
\hline topic231 & 0.00012838 & $6.9432 \mathrm{e}-05$ & $1.60573 \mathrm{e}+06$ & $7.3572 \mathrm{e}-115$ & Reject \\
\hline topic232 & $9.5982 \mathrm{e}-05$ & $2.9754 \mathrm{e}-05$ & $1.6984 \mathrm{e}+06$ & $7.8778 \mathrm{e}-96$ & Reject \\
\hline topic233 & 0.00017673 & 0.0001145 & $1.84072 \mathrm{e}+06$ & $3.2229 \mathrm{e}-69$ & Reject \\
\hline topic234 & 0.00012173 & 0.00012849 & $2.51423 \mathrm{e}+06$ & 0.0054485 & Reject \\
\hline topic235 & $4.5425 \mathrm{e}-05$ & $3.0777 \mathrm{e}-05$ & $2.17443 \mathrm{e}+06$ & $2.1743 \mathrm{e}-23$ & Reject \\
\hline
\end{tabular}


Table A1 - Continued from previous page

\begin{tabular}{|c|c|c|c|c|c|}
\hline Feature & EN median & FL median & u-value & p-value & $\mathbf{H}_{0}$ \\
\hline topic236 & $1.0911 \mathrm{e}-05$ & $2.3793 \mathrm{e}-05$ & $1.82685 \mathrm{e}+06$ & $6.5999 \mathrm{e}-65$ & Reject \\
\hline topic237 & 0.00013158 & $9.7533 \mathrm{e}-05$ & $2.00317 \mathrm{e}+06$ & $1.7624 \mathrm{e}-44$ & Reject \\
\hline topic238 & 0.000198 & 0.00015126 & $2.06207 \mathrm{e}+06$ & $5.5225 \mathrm{e}-37$ & Reject \\
\hline topic239 & $7.6242 \mathrm{e}-05$ & $6.5852 \mathrm{e}-05$ & $2.39643 \mathrm{e}+06$ & $1.1533 \mathrm{e}-07$ & Reject \\
\hline topic240 & $9.5048 \mathrm{e}-05$ & 9.9487 e-05 & $2.52221 \mathrm{e}+06$ & 0.008331 & Reject \\
\hline topic241 & $5.6724 \mathrm{e}-05$ & $5.1384 \mathrm{e}-05$ & $2.41981 \mathrm{e}+06$ & $1.4586 \mathrm{e}-06$ & Reject \\
\hline topic242 & $8.9502 \mathrm{e}-05$ & $4.7419 \mathrm{e}-05$ & $1.60095 \mathrm{e}+06$ & $1.8137 \mathrm{e}-116$ & Reject \\
\hline topic243 & $8.8398 \mathrm{e}-05$ & $3.9025 \mathrm{e}-05$ & $1.21527 \mathrm{e}+06$ & $8.071 \mathrm{e}-218$ & Reject \\
\hline topic244 & 0.00012174 & 0.00011233 & $2.44047 \mathrm{e}+06$ & $1.9195 \mathrm{e}-05$ & Reject \\
\hline topic245 & 0.00011092 & $7.4119 \mathrm{e}-05$ & $2.01747 \mathrm{e}+06$ & $1.4602 \mathrm{e}-42$ & Reject \\
\hline topic246 & 0.0001021 & 0.00011233 & $2.40452 \mathrm{e}+06$ & $2.6164 \mathrm{e}-07$ & Reject \\
\hline topic247 & 0.00013101 & $7.7636 \mathrm{e}-05$ & $1.72341 \mathrm{e}+06$ & $1.0528 \mathrm{e}-90$ & Reject \\
\hline topic248 & 0.00011277 & 0.00011312 & $2.61067 \mathrm{e}+06$ & 0.35522 & Accept \\
\hline topic249 & $9.9454 \mathrm{e}-05$ & $7.128 \mathrm{e}-05$ & $2.04418 \mathrm{e}+06$ & $1.0258 \mathrm{e}-38$ & Reject \\
\hline topic250 & 0.00011881 & $9.2757 \mathrm{e}-05$ & $2.24893 \mathrm{e}+06$ & $1.0716 \mathrm{e}-17$ & Reject \\
\hline topic251 & $8.5233 \mathrm{e}-05$ & $7.2467 \mathrm{e}-05$ & $2.13036 \mathrm{e}+06$ & $5.88 \mathrm{e}-29$ & Reject \\
\hline topic252 & $4.1287 \mathrm{e}-05$ & $3.0559 \mathrm{e}-05$ & $2.17247 \mathrm{e}+06$ & $2.2456 \mathrm{e}-24$ & Reject \\
\hline topic253 & 0.00013634 & $7.2357 \mathrm{e}-05$ & $1.7404 \mathrm{e}+06$ & $2.0794 \mathrm{e}-87$ & Reject \\
\hline topic254 & $7.026 \mathrm{e}-05$ & $3.43 e-05$ & $1.66414 \mathrm{e}+06$ & $2.5376 \mathrm{e}-102$ & Reject \\
\hline topic255 & $5.2416 \mathrm{e}-05$ & $3.4648 \mathrm{e}-05$ & $1.8551 \mathrm{e}+06$ & $8.9447 \mathrm{e}-67$ & Reject \\
\hline topic256 & 0.00018353 & 0.0001504 & $2.12935 \mathrm{e}+06$ & $3.5278 \mathrm{e}-29$ & Reject \\
\hline topic257 & $9.486 \mathrm{e}-05$ & $8.3015 \mathrm{e}-05$ & $2.30097 \mathrm{e}+06$ & $1.4234 \mathrm{e}-13$ & Reject \\
\hline topic258 & 0.00012739 & 0.00014934 & $2.39961 \mathrm{e}+06$ & $2.1874 \mathrm{e}-07$ & Reject \\
\hline topic259 & $5.9801 \mathrm{e}-05$ & $3.7294 \mathrm{e}-05$ & $1.66187 \mathrm{e}+06$ & $3.6804 \mathrm{e}-103$ & Reject \\
\hline topic260 & $8.3552 \mathrm{e}-05$ & $8.3558 \mathrm{e}-05$ & $2.57446 \mathrm{e}+06$ & 0.12497 & Accept \\
\hline topic261 & 0.00020352 & 0.00017582 & $2.23969 \mathrm{e}+06$ & $2.2 \mathrm{e}-18$ & Reject \\
\hline topic262 & 0.00011035 & $7.2684 \mathrm{e}-05$ & $1.62405 \mathrm{e}+06$ & $2.1612 \mathrm{e}-111$ & Reject \\
\hline topic263 & 0.00012562 & $8.1926 \mathrm{e}-05$ & $1.98129 \mathrm{e}+06$ & $7.7032 \mathrm{e}-47$ & Reject \\
\hline topic264 & 0.00014818 & $7.4241 \mathrm{e}-05$ & $1.62209 \mathrm{e}+06$ & $1.1987 \mathrm{e}-111$ & Reject \\
\hline topic265 & $2.5377 \mathrm{e}-05$ & $1.3968 \mathrm{e}-05$ & $1.86688 \mathrm{e}+06$ & $4.3803 \mathrm{e}-64$ & Reject \\
\hline topic266 & $2.5566 \mathrm{e}-05$ & $2.4408 \mathrm{e}-05$ & $2.46418 \mathrm{e}+06$ & 0.00016681 & Reject \\
\hline topic267 & $9.8021 \mathrm{e}-06$ & $1.4152 \mathrm{e}-06$ & $1.5246 \mathrm{e}+06$ & $4.0392 \mathrm{e}-133$ & Reject \\
\hline topic268 & 0.00010433 & $7.0144 \mathrm{e}-05$ & $2.08457 \mathrm{e}+06$ & $4.8937 \mathrm{e}-34$ & Reject \\
\hline topic269 & 0.00013114 & $7.8728 \mathrm{e}-05$ & $1.67089 \mathrm{e}+06$ & $4.3782 \mathrm{e}-101$ & Reject \\
\hline topic270 & 0.00012256 & 0.00011279 & $2.41672 \mathrm{e}+06$ & $1.1772 \mathrm{e}-06$ & Reject \\
\hline topic271 & 0.00014389 & 7.5037e-05 & $2.00678 \mathrm{e}+06$ & $7.1677 \mathrm{e}-44$ & Reject \\
\hline topic272 & $5.1701 \mathrm{e}-06$ & $1.1671 \mathrm{e}-06$ & $1.87711 \mathrm{e}+06$ & $1.6304 \mathrm{e}-61$ & Reject \\
\hline topic273 & 0.0001332 & 0.00010873 & $2.37867 \mathrm{e}+06$ & $1.9367 \mathrm{e}-08$ & Reject \\
\hline topic274 & 0.00015183 & 0.00013584 & $2.43253 \mathrm{e}+06$ & $6.9036 \mathrm{e}-06$ & Reject \\
\hline topic275 & 0.00011692 & $9.0914 \mathrm{e}-05$ & $2.16365 \mathrm{e}+06$ & $1.8171 \mathrm{e}-25$ & Reject \\
\hline topic276 & 0.00017296 & 0.00011773 & $1.78384 \mathrm{e}+06$ & $2.9976 \mathrm{e}-79$ & Reject \\
\hline topic277 & 0.0001602 & 0.00024516 & $1.81913 \mathrm{e}+06$ & $4.2619 \mathrm{e}-73$ & Reject \\
\hline
\end{tabular}


Table A1 - Continued from previous page

\begin{tabular}{|c|c|c|c|c|c|}
\hline Feature & EN median & FL median & u-value & p-value & $\mathbf{H}_{0}$ \\
\hline topic278 & 0.0001475 & $9.114 \mathrm{e}-05$ & $1.72708 \mathrm{e}+06$ & 7.9131e-90 & Reject \\
\hline topic279 & $7.699 \mathrm{e}-05$ & $5.0709 \mathrm{e}-05$ & $2.22474 \mathrm{e}+06$ & $2.0474 \mathrm{e}-19$ & Reject \\
\hline topic280 & $4.5189 \mathrm{e}-05$ & $2.9624 \mathrm{e}-05$ & $2.13872 \mathrm{e}+06$ & $1.1995 \mathrm{e}-25$ & Reject \\
\hline topic281 & $8.8321 \mathrm{e}-05$ & $5.2586 \mathrm{e}-05$ & $2.1122 \mathrm{e}+06$ & $9.6468 \mathrm{e}-31$ & Reject \\
\hline topic282 & 0.00020098 & 0.00019042 & $2.45612 \mathrm{e}+06$ & $5.5055 \mathrm{e}-05$ & Reject \\
\hline topic283 & $9.1762 \mathrm{e}-05$ & $9.5635 \mathrm{e}-05$ & $2.59107 \mathrm{e}+06$ & 0.21005 & Accept \\
\hline topic284 & 0.00010219 & $8.5671 \mathrm{e}-05$ & $2.38866 \mathrm{e}+06$ & $7.4919 \mathrm{e}-08$ & Reject \\
\hline topic285 & 0.00020664 & 0.00034847 & $1.64262 \mathrm{e}+06$ & $1.4078 \mathrm{e}-107$ & Reject \\
\hline topic286 & $3.9795 \mathrm{e}-05$ & $3.8791 \mathrm{e}-05$ & $2.4935 \mathrm{e}+06$ & 0.0019773 & Reject \\
\hline topic287 & $6.948 \mathrm{e}-05$ & $5.1316 \mathrm{e}-05$ & $1.98779 \mathrm{e}+06$ & $9.9118 \mathrm{e}-47$ & Reject \\
\hline topic288 & $8.7883 \mathrm{e}-05$ & $5.8525 \mathrm{e}-05$ & $1.37573 \mathrm{e}+06$ & $1.3329 \mathrm{e}-171$ & Reject \\
\hline topic289 & 0.00014128 & 0.00017361 & $2.26684 \mathrm{e}+06$ & $3.673 \mathrm{e}-16$ & Reject \\
\hline topic290 & 0.00014036 & 0.00011911 & $2.45136 \mathrm{e}+06$ & $5.9395 \mathrm{e}-05$ & Reject \\
\hline topic291 & $7.3326 \mathrm{e}-05$ & $4.9962 \mathrm{e}-05$ & $1.871 \mathrm{e}+06$ & $5.3264 \mathrm{e}-64$ & Reject \\
\hline topic292 & 0.00014705 & 0.00012607 & $2.23393 \mathrm{e}+06$ & $5.7189 \mathrm{e}-19$ & Reject \\
\hline topic293 & 0.00011795 & 0.00010541 & $2.41376 \mathrm{e}+06$ & $9.6168 \mathrm{e}-07$ & Reject \\
\hline topic294 & 0.00011245 & $7.7858 \mathrm{e}-05$ & $1.70386 \mathrm{e}+06$ & $9.8595 \mathrm{e}-95$ & Reject \\
\hline topic295 & $7.9142 \mathrm{e}-05$ & $6.6949 \mathrm{e}-05$ & $2.39363 \mathrm{e}+06$ & $8.2402 \mathrm{e}-08$ & Reject \\
\hline topic296 & 0.00012942 & 0.00011668 & $2.42439 \mathrm{e}+06$ & $3.8042 \mathrm{e}-06$ & Reject \\
\hline topic297 & $6.4771 \mathrm{e}-05$ & $3.8418 \mathrm{e}-05$ & $1.73654 \mathrm{e}+06$ & $3.6262 \mathrm{e}-88$ & Reject \\
\hline topic298 & $3.4849 \mathrm{e}-05$ & $1.6738 \mathrm{e}-05$ & $1.85739 \mathrm{e}+06$ & $4.1652 \mathrm{e}-66$ & Reject \\
\hline topic299 & $7.7021 \mathrm{e}-05$ & $7.8144 \mathrm{e}-05$ & $2.61301 \mathrm{e}+06$ & 0.37571 & Accept \\
\hline topic300 & 0.00017635 & 0.0001297 & $2.4127 \mathrm{e}+06$ & 0.00085957 & Reject \\
\hline topic301 & 0.00016564 & 0.00012536 & $1.97279 \mathrm{e}+06$ & $1.0582 \mathrm{e}-48$ & Reject \\
\hline topic302 & 0.00011998 & 0.00015827 & $2.21255 \mathrm{e}+06$ & $9.0367 \mathrm{e}-21$ & Reject \\
\hline topic303 & 0.00014663 & 0.00015697 & $2.48636 \mathrm{e}+06$ & 0.00070382 & Reject \\
\hline topic304 & 0.00012488 & $9.143 \mathrm{e}-05$ & $2.16948 \mathrm{e}+06$ & $7.0114 \mathrm{e}-25$ & Reject \\
\hline topic305 & 0.00010374 & 0.00011318 & $2.51675 \mathrm{e}+06$ & 0.0068285 & Reject \\
\hline topic306 & $9.2333 \mathrm{e}-05$ & $8.9867 \mathrm{e}-05$ & $2.5522 \mathrm{e}+06$ & 0.044423 & Reject \\
\hline topic307 & $7.3953 \mathrm{e}-05$ & $7.2055 \mathrm{e}-05$ & $2.51852 \mathrm{e}+06$ & 0.0070899 & Reject \\
\hline topic308 & 0.00011071 & 0.00010799 & $2.59273 \mathrm{e}+06$ & 0.22089 & Accept \\
\hline topic309 & $9.9786 \mathrm{e}-05$ & 0.00010654 & $2.52597 \mathrm{e}+06$ & 0.014612 & Reject \\
\hline topic310 & 0.00014106 & 0.00011244 & $2.33105 \mathrm{e}+06$ & $3.7529 \mathrm{e}-11$ & Reject \\
\hline topic311 & 0.00014794 & 0.00014771 & $2.55278 \mathrm{e}+06$ & 0.045853 & Reject \\
\hline topic312 & $8.5507 \mathrm{e}-05$ & $2.2795 \mathrm{e}-05$ & 973390 & $1.9594 \mathrm{e}-298$ & Reject \\
\hline topic313 & $2.4752 \mathrm{e}-05$ & $1.7764 \mathrm{e}-05$ & $2.17467 \mathrm{e}+06$ & $2.303 \mathrm{e}-24$ & Reject \\
\hline topic314 & 0.00011944 & $5.8465 \mathrm{e}-05$ & $1.58679 \mathrm{e}+06$ & $6.8055 \mathrm{e}-119$ & Reject \\
\hline topic315 & $5.156 \mathrm{e}-05$ & $2.4496 \mathrm{e}-05$ & $1.55255 \mathrm{e}+06$ & $1.3551 \mathrm{e}-125$ & Reject \\
\hline topic316 & $2.3654 \mathrm{e}-05$ & $8.1678 \mathrm{e}-06$ & $1.69521 \mathrm{e}+06$ & $8.9359 \mathrm{e}-96$ & Reject \\
\hline topic317 & $2.7732 \mathrm{e}-08$ & $8.3569 \mathrm{e}-08$ & $1.0416 \mathrm{e}+06$ & $6.388 \mathrm{e}-43$ & Reject \\
\hline topic318 & 0.00015376 & 0.00017872 & $2.31534 \mathrm{e}+06$ & $1.2324 \mathrm{e}-12$ & Reject \\
\hline topic319 & 0.00014556 & $8.2452 \mathrm{e}-05$ & $1.79743 \mathrm{e}+06$ & $5.9583 \mathrm{e}-77$ & Reject \\
\hline
\end{tabular}


Table A1 - Continued from previous page

\begin{tabular}{|c|c|c|c|c|c|}
\hline Feature & EN median & FL median & u-value & p-value & $\mathrm{H}_{\mathbf{0}}$ \\
\hline topic320 & 0.00011198 & $9.0273 \mathrm{e}-05$ & $2.25635 \mathrm{e}+06$ & $6.5577 \mathrm{e}-17$ & Reject \\
\hline topic321 & 0.00017205 & 0.00020118 & $2.39483 \mathrm{e}+06$ & $1.076 \mathrm{e}-07$ & Reject \\
\hline topic322 & $6.6753 \mathrm{e}-05$ & $3.8273 \mathrm{e}-05$ & $1.53191 \mathrm{e}+06$ & $2.4995 \mathrm{e}-132$ & Reject \\
\hline topic323 & 0.00015989 & 1927 & $2.11342 \mathrm{e}+06$ & $1.0389 \mathrm{e}-30$ & Reject \\
\hline topic324 & 0.00011575 & $7.1518 \mathrm{e}-05$ & $2.07974 \mathrm{e}+06$ & $8.4377 \mathrm{e}-34$ & Reject \\
\hline topic325 & $4.2565 \mathrm{e}-05$ & $3.9351 \mathrm{e}-06$ & $1.0561 \mathrm{e}+06$ & $3.0117 \mathrm{e}-263$ & Reject \\
\hline topic326 & $9.9814 \mathrm{e}-05$ & $6.237 \mathrm{e}-05$ & $2.0645 \mathrm{e}+06$ & $1.8895 \mathrm{e}-36$ & Reject \\
\hline topic327 & 0.0001193 & $3.0114 \mathrm{e}-05$ & $1.21646 \mathrm{e}+06$ & $6.1641 \mathrm{e}-216$ & Reject \\
\hline topi & 0.00016103 & 0.00015671 & $2.57726 \mathrm{e}+06$ & 0.13781 & Accept \\
\hline topic329 & $8.2718 \mathrm{e}-05$ & $5.193 \mathrm{e}-05$ & $2.27495 \mathrm{e}+06$ & $1.9248 \mathrm{e}-15$ & Reject \\
\hline topic330 & $9.0184 \mathrm{e}-05$ & $3.1622 \mathrm{e}-05$ & $1.45267 \mathrm{e}+06$ & $1.3489 \mathrm{e}-151$ & Reject \\
\hline topi & 0.00018446 & 0.00018262 & $2.56155 \mathrm{e}+06$ & 0.067987 & Accept \\
\hline topic332 & 0.00012222 & $8.3534 \mathrm{e}-05$ & $1.67371 \mathrm{e}+06$ & $6.9992 \mathrm{e}-101$ & Reject \\
\hline & 0.00014553 & 13995 & $2.55883 \mathrm{e}+06$ & 0.066626 & Accept \\
\hline & 0.00010207 & & $1.93022 \mathrm{e}+06$ & $1.1279 \mathrm{e}-54$ & \\
\hline 335 & 0.00012163 & $8.7037 \mathrm{e}-05$ & $1.92371 \mathrm{e}+06$ & $2.2779 \mathrm{e}-55$ & Reject \\
\hline topi & $2281 \mathrm{e}-05$ & $1 \mathrm{e}-05$ & $1.28974 \mathrm{e}+06$ & $1.7706 \mathrm{e}-192$ & Reject \\
\hline topic337 & 0.00013097 & $8.5104 \mathrm{e}-05$ & $2.04206 \mathrm{e}+06$ & $5.1152 \mathrm{e}-39$ & Reject \\
\hline & e- 05 & $7 e-05$ & $1.8851 \mathrm{e}+06$ & $2.6955 \mathrm{e}-61$ & Reject \\
\hline & & & & & \\
\hline topi & $7.2582 \mathrm{e}-05$ & $5.0618 \mathrm{e}-05$ & $2.08759 \mathrm{e}+06$ & $5.7277 \mathrm{e}-33$ & Reject \\
\hline topi & $8.5107 \mathrm{e}-05$ & $9 \mathrm{e}-05$ & $2.39216 \mathrm{e}+06$ & $6.8974 \mathrm{e}-08$ & Reject \\
\hline topi & $1.9642 \mathrm{e}-05$ & $9 e-05$ & $1.89586 \mathrm{e}+06$ & $3.8257 \mathrm{e}-60$ & Reject \\
\hline top & 0.00014339 & $1 \mathrm{e}-05$ & $2.0025 \mathrm{e}+06$ & $1.1635 \mathrm{e}-43$ & Reject \\
\hline & $5949 \mathrm{e}-05$ & & $2.52197 \mathrm{e}+06$ & 599 & Reject \\
\hline top & $9.0413 \mathrm{e}-05$ & $1 \mathrm{e}-05$ & $1.355 \mathrm{e}+06$ & $1.2645 \mathrm{e}-176$ & Reject \\
\hline 346 & e-05 & $2 \mathrm{e}-05$ & $1.83628 \mathrm{e}+06$ & $2.7819 \mathrm{e}-70$ & Reject \\
\hline & 0.00016311 & $7.4148 \mathrm{e}-05$ & $1.51944 \mathrm{e}+06$ & $8.9552 \mathrm{e}-133$ & Reject \\
\hline top & 0.00010441 & $6.6214 \mathrm{e}-05$ & $1.94775 \mathrm{e}+06$ & $3.4485 \mathrm{e}-52$ & Reject \\
\hline & 1258 & & $1.82312 \mathrm{e}+06$ & $3.0863 \mathrm{e}-72$ & Reject \\
\hline top & $8.7593 \mathrm{e}-05$ & $6.2101 \mathrm{e}-05$ & $2.01374 \mathrm{e}+06$ & $4.6567 \mathrm{e}-43$ & Reject \\
\hline topic351 & 0.00011332 & $7.7796 \mathrm{e}-05$ & $1.9655 \mathrm{e}+06$ & $1.7757 \mathrm{e}-49$ & Reject \\
\hline & 0.00 & 0.00018718 & $2.3902 \mathrm{e}+06$ & $9.1246 \mathrm{e}-08$ & Reject \\
\hline top & $7.4849 \mathrm{e}-05$ & $4.5193 \mathrm{e}-05$ & $2.09641 \mathrm{e}+06$ & $2.0324 \mathrm{e}-32$ & Reject \\
\hline topic354 & 0.00012021 & 0.00012049 & $2.59256 \mathrm{e}+06$ & 0.21201 & Accept \\
\hline & 0.00011997 & $6.6609 \mathrm{e}-05$ & $1.60286 \mathrm{e}+06$ & $2.6291 \mathrm{e}-115$ & Reject \\
\hline topic356 & $6.9722 \mathrm{e}-05$ & $5.8065 \mathrm{e}-05$ & $2.32674 \mathrm{e}+06$ & $1.6418 \mathrm{e}-11$ & Reject \\
\hline 357 & 0.00011431 & 0.00012887 & $2.43885 \mathrm{e}+06$ & $1.0588 \mathrm{e}-05$ & Reject \\
\hline & 0.00021259 & 0.00015786 & $1.81639 \mathrm{e}+06$ & $9.664 \mathrm{e}-74$ & Reject \\
\hline topic359 & 0.00010313 & $3.4122 \mathrm{e}-05$ & $1.62072 \mathrm{e}+06$ & $6.2919 \mathrm{e}-112$ & Reject \\
\hline & 0.00016387 & 0.00013154 & $2.18565 \mathrm{e}+06$ & $2.6709 \mathrm{e}-23$ & Reject \\
\hline topic361 & 0.00012224 & $8.2631 \mathrm{e}-05$ & $2.1454 \mathrm{e}+06$ & $3.8348 \mathrm{e}-27$ & Reject \\
\hline
\end{tabular}


Table A1 - Continued from previous page

\begin{tabular}{|c|c|c|c|c|c|}
\hline Feature & EN median & FL median & u-value & p-value & $\mathbf{H}_{0}$ \\
\hline topic362 & $1.1386 \mathrm{e}-05$ & $1.0268 \mathrm{e}-05$ & $2.48564 \mathrm{e}+06$ & 0.00066553 & Reject \\
\hline topic363 & $7.5499 \mathrm{e}-05$ & $6.7753 \mathrm{e}-05$ & $2.37527 \mathrm{e}+06$ & $8.2792 \mathrm{e}-09$ & Reject \\
\hline topic364 & $3.8688 \mathrm{e}-05$ & $6.064 \mathrm{e}-05$ & $2.11767 \mathrm{e}+06$ & $6.0797 \mathrm{e}-29$ & Reject \\
\hline topic365 & $9.6917 \mathrm{e}-05$ & $6.9086 \mathrm{e}-05$ & $2.06351 \mathrm{e}+06$ & $1.1038 \mathrm{e}-36$ & Reject \\
\hline topic366 & $2.929 \mathrm{e}-05$ & $2.0754 \mathrm{e}-05$ & $2.14329 \mathrm{e}+06$ & $3.7116 \mathrm{e}-27$ & Reject \\
\hline topic367 & 0.00010264 & $5.516 \mathrm{e}-05$ & $1.82967 \mathrm{e}+06$ & $4.2274 \mathrm{e}-71$ & Reject \\
\hline topic368 & $4.4843 \mathrm{e}-05$ & $1.3145 \mathrm{e}-05$ & $1.46608 \mathrm{e}+06$ & $4.8107 \mathrm{e}-148$ & Reject \\
\hline topic369 & $9.9088 \mathrm{e}-05$ & $5.6429 \mathrm{e}-05$ & $1.95713 \mathrm{e}+06$ & 7.6931e-51 & Reject \\
\hline topic370 & 0.00012629 & 0.00012322 & $2.59155 \mathrm{e}+06$ & 0.19814 & Accept \\
\hline topic371 & 0.00010333 & 0.00011238 & $2.47055 \mathrm{e}+06$ & 0.00021566 & Reject \\
\hline topic372 & $6.4555 \mathrm{e}-05$ & $4.1585 \mathrm{e}-05$ & $1.769 \mathrm{e}+06$ & $5.3099 \mathrm{e}-82$ & Reject \\
\hline topic373 & 0.00017048 & 0.00011976 & $2.16897 \mathrm{e}+06$ & $2.0228 \mathrm{e}-24$ & Reject \\
\hline topic374 & 0.00012822 & 0.00011256 & $2.30638 \mathrm{e}+06$ & $3.4632 \mathrm{e}-13$ & Reject \\
\hline topic375 & 0.00013535 & 0.00010017 & $1.9481 \mathrm{e}+06$ & $2.0093 \mathrm{e}-52$ & Reject \\
\hline topic376 & 0.00011148 & $7.783 \mathrm{e}-05$ & $1.78816 \mathrm{e}+06$ & $8.5185 \mathrm{e}-79$ & Reject \\
\hline topic377 & 0.00012713 & $8.496 \mathrm{e}-05$ & $2.06798 \mathrm{e}+06$ & $1.1708 \mathrm{e}-35$ & Reject \\
\hline topic378 & 0.00010562 & $5.7975 \mathrm{e}-05$ & $1.91698 \mathrm{e}+06$ & $2.0014 \mathrm{e}-56$ & Reject \\
\hline topic379 & 0.00014129 & 0.00010649 & $2.06811 \mathrm{e}+06$ & 7.0974e-36 & Reject \\
\hline topic380 & $6.2499 \mathrm{e}-05$ & $3.3378 \mathrm{e}-05$ & $1.81627 \mathrm{e}+06$ & $1.2961 \mathrm{e}-73$ & Reject \\
\hline topic381 & 0.00011165 & $5.3839 \mathrm{e}-05$ & $1.55523 \mathrm{e}+06$ & $6.6278 \mathrm{e}-126$ & Reject \\
\hline topic382 & 0.00013523 & 0.0001946 & $1.97068 \mathrm{e}+06$ & $5.2879 \mathrm{e}-49$ & Reject \\
\hline topic383 & $3.2517 \mathrm{e}-06$ & $9.0716 \mathrm{e}-06$ & 84829 & $2.7468 \mathrm{e}-28$ & Reject \\
\hline topic384 & 0.00015148 & 0.00011227 & $2.12991 \mathrm{e}+06$ & $3.9752 \mathrm{e}-28$ & Reject \\
\hline topic385 & $8.759 \mathrm{e}-05$ & $3.1436 \mathrm{e}-05$ & $1.19166 \mathrm{e}+06$ & $1.1995 \mathrm{e}-224$ & Reject \\
\hline topic386 & 0.00013747 & $8.0058 \mathrm{e}-05$ & $1.89133 \mathrm{e}+06$ & $3.0303 \mathrm{e}-60$ & Reject \\
\hline topic387 & 0.00017728 & 0.00023118 & $2.1358 \mathrm{e}+06$ & $1.7561 \mathrm{e}-28$ & Reject \\
\hline topic388 & $7.8978 \mathrm{e}-05$ & $6.6503 \mathrm{e}-05$ & $2.36021 \mathrm{e}+06$ & $1.7262 \mathrm{e}-09$ & Reject \\
\hline topic389 & $7.1892 \mathrm{e}-05$ & $3.0042 \mathrm{e}-05$ & $1.1386 \mathrm{e}+06$ & $4.5584 \mathrm{e}-242$ & Reject \\
\hline topic390 & $7.7281 \mathrm{e}-05$ & $5.1667 \mathrm{e}-05$ & $1.93263 \mathrm{e}+06$ & $1.8639 \mathrm{e}-54$ & Reject \\
\hline topic391 & 0.00014469 & $9.7105 \mathrm{e}-05$ & $2.01942 \mathrm{e}+06$ & $4.7093 \mathrm{e}-42$ & Reject \\
\hline topic392 & 0.00013901 & 0.00015955 & $2.35864 \mathrm{e}+06$ & $1.1985 \mathrm{e}-09$ & Reject \\
\hline topic393 & 0.00012587 & $9.5491 \mathrm{e}-05$ & $2.19973 \mathrm{e}+06$ & $1.3581 \mathrm{e}-21$ & Reject \\
\hline topic394 & $3.3598 \mathrm{e}-05$ & $6.9453 \mathrm{e}-05$ & $1.83228 \mathrm{e}+06$ & $3.2286 \mathrm{e}-65$ & Reject \\
\hline topic395 & 0.00010412 & $7.2103 \mathrm{e}-05$ & $2.12435 \mathrm{e}+06$ & $2.1315 \mathrm{e}-29$ & Reject \\
\hline topic396 & 7.6117e-05 & $9.5607 \mathrm{e}-05$ & $2.43439 \mathrm{e}+06$ & $7.5758 \mathrm{e}-06$ & Reject \\
\hline topic397 & 0.00012741 & $7.132 \mathrm{e}-05$ & $1.85329 \mathrm{e}+06$ & $2.9485 \mathrm{e}-65$ & Reject \\
\hline topic398 & 0.00011216 & $8.5407 \mathrm{e}-05$ & $1.99522 \mathrm{e}+06$ & $1.4447 \mathrm{e}-45$ & Reject \\
\hline topic399 & $5.6838 \mathrm{e}-05$ & $3.3047 \mathrm{e}-05$ & $1.68706 \mathrm{e}+06$ & $2.6326 \mathrm{e}-98$ & Reject \\
\hline topic400 & 0.0001308 & 0.00010443 & $2.14798 \mathrm{e}+06$ & 7.1263e-27 & Reject \\
\hline topic401 & 0.00013071 & $8.6943 \mathrm{e}-05$ & $2.04264 \mathrm{e}+06$ & $8.7554 \mathrm{e}-39$ & Reject \\
\hline topic402 & 0.00012052 & 0.00011187 & $2.5242 \mathrm{e}+06$ & 0.010072 & Reject \\
\hline topic403 & $5.2545 \mathrm{e}-05$ & $5.3123 \mathrm{e}-05$ & $2.56353 \mathrm{e}+06$ & 0.073673 & Accept \\
\hline
\end{tabular}


Table A1 - Continued from previous page

\begin{tabular}{|c|c|c|c|c|c|}
\hline Feature & EN median & FL median & u-value & p-value & $\mathbf{H}_{0}$ \\
\hline topic404 & $9.0033 \mathrm{e}-05$ & $8.9209 \mathrm{e}-05$ & $2.62472 \mathrm{e}+06$ & 0.46627 & Accept \\
\hline topic405 & 0.00010055 & $3.979 \mathrm{e}-05$ & $1.83091 \mathrm{e}+06$ & $4.0806 \mathrm{e}-70$ & Reject \\
\hline topic406 & $9.7999 \mathrm{e}-05$ & $6.5547 \mathrm{e}-05$ & $2.12674 \mathrm{e}+06$ & $3.89 \mathrm{e}-29$ & Reject \\
\hline topic407 & $7.0035 \mathrm{e}-05$ & $6.5935 \mathrm{e}-05$ & $2.51953 \mathrm{e}+06$ & 0.007592 & Reject \\
\hline topic408 & $5.3263 \mathrm{e}-05$ & $5.9762 \mathrm{e}-05$ & $2.5029 \mathrm{e}+06$ & 0.0032067 & Reject \\
\hline topic409 & 0.00013512 & 0.00011309 & $2.19828 \mathrm{e}+06$ & $3.4652 \mathrm{e}-22$ & Reject \\
\hline topic410 & 0.00014888 & 0.00012551 & $2.26126 \mathrm{e}+06$ & $1.3172 \mathrm{e}-16$ & Reject \\
\hline topic411 & 0.00011939 & 0.00013951 & $2.40407 \mathrm{e}+06$ & $2.8286 \mathrm{e}-07$ & Reject \\
\hline topic412 & 0.00016077 & 0.00013274 & $2.4006 \mathrm{e}+06$ & $1.8891 \mathrm{e}-07$ & Reject \\
\hline topic413 & $8.8121 \mathrm{e}-05$ & $9.8216 \mathrm{e}-05$ & $2.48975 \mathrm{e}+06$ & 0.001076 & Reject \\
\hline topic414 & $9.3237 \mathrm{e}-05$ & $6.1287 \mathrm{e}-05$ & $1.99109 \mathrm{e}+06$ & $9.685 \mathrm{e}-46$ & Reject \\
\hline topic415 & 0.00013625 & $8.2025 \mathrm{e}-05$ & $1.6387 \mathrm{e}+06$ & $1.6529 \mathrm{e}-107$ & Reject \\
\hline topic416 & $9.3967 \mathrm{e}-05$ & $5.5222 \mathrm{e}-05$ & $1.92805 \mathrm{e}+06$ & $6.9829 \mathrm{e}-55$ & Reject \\
\hline topic417 & 0.00018302 & 02023 & $2.42675 \mathrm{e}+06$ & $6.748 \mathrm{e}-06$ & Reject \\
\hline top & $8.9194 \mathrm{e}-05$ & & $2.48655 \mathrm{e}+06$ & 542 & \\
\hline topic419 & 0.00014145 & 0.00010013 & $2.1329 \mathrm{e}+06$ & $1.1065 \mathrm{e}-28$ & Reject \\
\hline topic420 & 0.00015164 & 0.00010656 & $1.96575 \mathrm{e}+06$ & $1.2848 \mathrm{e}-48$ & Reject \\
\hline topic421 & 0.00015062 & 0.00011223 & $2.38081 \mathrm{e}+06$ & $2.5695 \mathrm{e}-08$ & Reject \\
\hline topic422 & 0.00012136 & $e-05$ & $1.94768 \mathrm{e}+06$ & $2.356 \mathrm{e}-52$ & Reject \\
\hline & & & +06 & & \\
\hline topic424 & $2.9043 \mathrm{e}-05$ & $5.9085 \mathrm{e}-06$ & $1.47361 \mathrm{e}+06$ & $1.5635 \mathrm{e}-145$ & Reject \\
\hline topic 425 & $4.9174 \mathrm{e}-05$ & $1.3322 \mathrm{e}-05$ & $1.49456 \mathrm{e}+06$ & $6.154 \mathrm{e}-140$ & Reject \\
\hline topic426 & $8.5887 \mathrm{e}-05$ & $3 e-05$ & $2.49612 \mathrm{e}+06$ & 0.0018755 & Reject \\
\hline 427 & $8.6583 \mathrm{e}-05$ & $e-05$ & $1.3336 \mathrm{e}+06$ & $8.1169 \mathrm{e}-184$ & Reject \\
\hline & 0.000 & & $1.94667 \mathrm{e}+06$ & $1.722 \mathrm{e}-52$ & Reject \\
\hline topic429 & 0.00014977 & 0.00015426 & $2.56563 \mathrm{e}+06$ & 0.076907 & Accept \\
\hline topic430 & 0.00010926 & 0.00 & $2.54222 \mathrm{e}+06$ & 0.027309 & Reject \\
\hline topic431 & 0.00013541 & 0.00013707 & $2.60467 \mathrm{e}+06$ & 0.31692 & Accept \\
\hline 432 & $8.8671 \mathrm{e}-05$ & 0.00013338 & $1.99812 \mathrm{e}+06$ & $.6067 \mathrm{e}-45$ & Reject \\
\hline & 0.00010623 & 05 & $1.95088 \mathrm{e}+06$ & $9.9838 \mathrm{e}-52$ & Reject \\
\hline topic434 & $8.1318 \mathrm{e}-05$ & $5.5902 \mathrm{e}-06$ & 753790 & 0 & Reject \\
\hline topic435 & 0.00015097 & 0.00012394 & $2.23224 \mathrm{e}+06$ & $5.0539 \mathrm{e}-19$ & Reject \\
\hline & $3 e-05$ & $6.2008 \mathrm{e}-05$ & $2.22025 \mathrm{e}+06$ & $3.5876 \mathrm{e}-20$ & Reject \\
\hline 437 & 0.00014714 & 0.00010074 & $1.86556 \mathrm{e}+06$ & $1.3896 \mathrm{e}-64$ & Reject \\
\hline topic438 & 0.00010152 & $9.1629 \mathrm{e}-05$ & $2.4269 \mathrm{e}+06$ & $3.5044 \mathrm{e}-06$ & Reject \\
\hline & 0.00011003 & $5.1409 \mathrm{e}-05$ & $1.69746 \mathrm{e}+06$ & $1.1164 \mathrm{e}-95$ & Reject \\
\hline topic440 & 0.00012921 & $9.163 \mathrm{e}-05$ & $2.14135 \mathrm{e}+06$ & $8.8597 \mathrm{e}-28$ & Reject \\
\hline 441 & 0.00019171 & 0.0002079 & $2.53395 \mathrm{e}+06$ & 0.017583 & Reject \\
\hline topic442 & 0.00013881 & 0.00013985 & $2.61189 \mathrm{e}+06$ & 0.36623 & Accept \\
\hline topic443 & $9.3249 \mathrm{e}-05$ & $5.844 \mathrm{e}-05$ & $1.92653 \mathrm{e}+06$ & $5.9119 \mathrm{e}-55$ & Reject \\
\hline topic444 & $9.849 \mathrm{e}-05$ & $6.4548 \mathrm{e}-05$ & $1.95223 \mathrm{e}+06$ & $3.0216 \mathrm{e}-51$ & Reject \\
\hline topic445 & 0.00013002 & 0.0001679 & $2.08666 \mathrm{e}+06$ & $5.1017 \mathrm{e}-34$ & Reject \\
\hline
\end{tabular}


Table A1 - Continued from previous page

\begin{tabular}{|c|c|c|c|c|c|}
\hline Feature & EN median & FL median & u-value & p-value & $\mathbf{H}_{0}$ \\
\hline topic446 & 0.00016145 & 0.00017927 & $2.44964 \mathrm{e}+06$ & $3.3539 \mathrm{e}-05$ & Reject \\
\hline topic447 & 0.00011246 & 0.00011032 & $2.59863 \mathrm{e}+06$ & 0.24495 & Accept \\
\hline topic448 & $2.6405 \mathrm{e}-05$ & $5.4087 \mathrm{e}-05$ & $1.77831 \mathrm{e}+06$ & $2.5173 \mathrm{e}-55$ & Reject \\
\hline topic449 & $4.7243 \mathrm{e}-05$ & $1.4523 \mathrm{e}-05$ & $1.28623 \mathrm{e}+06$ & $1.0747 \mathrm{e}-196$ & Reject \\
\hline topic450 & 0.00010657 & $8.0983 \mathrm{e}-05$ & $2.06405 \mathrm{e}+06$ & $1.2539 \mathrm{e}-36$ & Reject \\
\hline topic451 & $5.8551 \mathrm{e}-05$ & $1.5933 \mathrm{e}-05$ & $1.76114 \mathrm{e}+06$ & $1.8796 \mathrm{e}-81$ & Reject \\
\hline topic452 & $7.1444 \mathrm{e}-05$ & $4.8285 \mathrm{e}-05$ & $2.10018 \mathrm{e}+06$ & $2.5698 \mathrm{e}-30$ & Reject \\
\hline topic453 & 0.00014039 & 0.00011314 & $2.16296 \mathrm{e}+06$ & $1.9751 \mathrm{e}-25$ & Reject \\
\hline topic454 & 0.00016736 & 0.000158 & $2.48134 \mathrm{e}+06$ & 0.00047461 & Reject \\
\hline topic455 & $7.7031 \mathrm{e}-05$ & 0.00026477 & $1.41412 \mathrm{e}+06$ & $4.1185 \mathrm{e}-144$ & Reject \\
\hline topic456 & 0.0001055 & $2.5112 \mathrm{e}-05$ & $1.07724 \mathrm{e}+06$ & $5.6396 \mathrm{e}-259$ & Reject \\
\hline topic457 & $8.6419 \mathrm{e}-05$ & $3.5984 \mathrm{e}-05$ & $1.27548 \mathrm{e}+06$ & $7.9596 \mathrm{e}-200$ & Reject \\
\hline topic458 & 0.00010251 & $9.2886 \mathrm{e}-05$ & $2.46494 \mathrm{e}+06$ & 0.00023574 & Reject \\
\hline topic459 & 0.00015062 & $8.4468 \mathrm{e}-05$ & $1.89437 \mathrm{e}+06$ & $2.2187 \mathrm{e}-60$ & Reject \\
\hline topic460 & $6.9982 \mathrm{e}-05$ & $6.0334 \mathrm{e}-05$ & $2.45505 \mathrm{e}+06$ & $8.2159 \mathrm{e}-05$ & Reject \\
\hline topic461 & 0.00010568 & $4.5311 \mathrm{e}-05$ & $1.76042 \mathrm{e}+06$ & $1.3745 \mathrm{e}-83$ & Reject \\
\hline topic462 & 0.00011429 & $9.1465 \mathrm{e}-05$ & $2.02196 \mathrm{e}+06$ & $4.2363 \mathrm{e}-42$ & Reject \\
\hline topic463 & 0.00025627 & 0.00023667 & $2.40228 \mathrm{e}+06$ & $2.2988 \mathrm{e}-07$ & Reject \\
\hline topic464 & $6.4159 \mathrm{e}-05$ & $2.8434 \mathrm{e}-05$ & $1.19593 \mathrm{e}+06$ & $3.2853 \mathrm{e}-224$ & Reject \\
\hline topic465 & $2.9812 \mathrm{e}-05$ & $2.6347 \mathrm{e}-05$ & $2.28701 \mathrm{e}+06$ & $1.342 \mathrm{e}-14$ & Reject \\
\hline topic466 & $7.6624 \mathrm{e}-05$ & $1.9105 \mathrm{e}-05$ & $1.28629 \mathrm{e}+06$ & $1.0648 \mathrm{e}-196$ & Reject \\
\hline topic467 & $9.6082 \mathrm{e}-05$ & $2.6683 \mathrm{e}-05$ & $1.2634 \mathrm{e}+06$ & $8.3055 \mathrm{e}-204$ & Reject \\
\hline topic468 & $8.7756 \mathrm{e}-05$ & $4.1479 \mathrm{e}-05$ & $1.88123 \mathrm{e}+06$ & $2.4658 \mathrm{e}-62$ & Reject \\
\hline topic469 & $6.7515 \mathrm{e}-05$ & $5.3091 \mathrm{e}-05$ & $2.31829 \mathrm{e}+06$ & $2.3358 \mathrm{e}-12$ & Reject \\
\hline topic470 & 0.00013004 & $8.9264 \mathrm{e}-05$ & $1.72985 \mathrm{e}+06$ & $1.3199 \mathrm{e}-89$ & Reject \\
\hline topic471 & 0.00012298 & $6.3417 \mathrm{e}-05$ & $1.5556 \mathrm{e}+06$ & $1.3508 \mathrm{e}-126$ & Reject \\
\hline topic472 & 0.00015051 & 0.00013843 & $2.36696 \mathrm{e}+06$ & $2.7378 \mathrm{e}-09$ & Reject \\
\hline topic473 & $4.5262 \mathrm{e}-05$ & $1.8307 \mathrm{e}-05$ & $1.79555 \mathrm{e}+06$ & $1.8659 \mathrm{e}-77$ & Reject \\
\hline topic474 & 0.0001323 & $7.5423 \mathrm{e}-05$ & $1.77806 \mathrm{e}+06$ & $3.8311 \mathrm{e}-80$ & Reject \\
\hline topic475 & 0.00013057 & 0.00013861 & $2.50788 \mathrm{e}+06$ & 0.0038843 & Reject \\
\hline topic476 & 0.00010156 & $8.845 \mathrm{e}-05$ & $2.41019 \mathrm{e}+06$ & $6.4618 \mathrm{e}-07$ & Reject \\
\hline topic477 & 0.00015755 & 0.00013848 & $2.26448 \mathrm{e}+06$ & $2.4283 \mathrm{e}-16$ & Reject \\
\hline topic478 & $8.2728 \mathrm{e}-05$ & $9.106 \mathrm{e}-05$ & $2.54007 \mathrm{e}+06$ & 0.027429 & Reject \\
\hline topic479 & $2.3888 \mathrm{e}-05$ & $2.6781 \mathrm{e}-05$ & $2.45642 \mathrm{e}+06$ & $6.217 \mathrm{e}-05$ & Reject \\
\hline topic480 & 0.00015931 & 0.00016882 & $2.54535 \mathrm{e}+06$ & 0.030181 & Reject \\
\hline topic481 & $8.9217 \mathrm{e}-05$ & $5.0091 \mathrm{e}-05$ & $1.90278 \mathrm{e}+06$ & $4.8852 \mathrm{e}-59$ & Reject \\
\hline topic482 & 0.00011918 & $6.9483 \mathrm{e}-05$ & $2.02956 \mathrm{e}+06$ & $1.8596 \mathrm{e}-40$ & Reject \\
\hline topic483 & 0.00012108 & 0.00010247 & $2.31736 \mathrm{e}+06$ & $1.6964 \mathrm{e}-12$ & Reject \\
\hline topic484 & $9.1311 \mathrm{e}-05$ & $4.1951 \mathrm{e}-05$ & $2.08503 \mathrm{e}+06$ & $1.7959 \mathrm{e}-31$ & Reject \\
\hline topic485 & $3.9423 \mathrm{e}-05$ & $1.1719 \mathrm{e}-05$ & $1.52004 \mathrm{e}+06$ & $3.6831 \mathrm{e}-135$ & Reject \\
\hline topic486 & 0.0001013 & $7.1546 \mathrm{e}-05$ & $1.9966 \mathrm{e}+06$ & $2.9634 \mathrm{e}-45$ & Reject \\
\hline topic487 & $6.7568 \mathrm{e}-05$ & $3.0406 \mathrm{e}-05$ & $1.72644 \mathrm{e}+06$ & $1.0021 \mathrm{e}-87$ & Reject \\
\hline
\end{tabular}


Table A1 - Continued from previous page

\begin{tabular}{|c|c|c|c|c|c|}
\hline Feature & EN median & FL median & u-value & p-value & $\mathrm{H}_{0}$ \\
\hline topic488 & $9.4283 \mathrm{e}-05$ & 0.00011347 & $2.31674 \mathrm{e}+06$ & $1.5372 \mathrm{e}-12$ & Reject \\
\hline topic489 & 0.00013982 & 0.00010221 & $2.05679 \mathrm{e}+06$ & $1.1524 \mathrm{e}-36$ & Reject \\
\hline topic490 & $3.5265 \mathrm{e}-06$ & $1.9862 \mathrm{e}-06$ & $1.85276 \mathrm{e}+06$ & $9.8686 \mathrm{e}-59$ & Reject \\
\hline topic491 & $6.4021 \mathrm{e}-05$ & $6.7368 \mathrm{e}-05$ & $2.56958 \mathrm{e}+06$ & 0.090415 & Accept \\
\hline topic492 & $8.0017 \mathrm{e}-05$ & $3.378 \mathrm{e}-05$ & $1.58760 \mathrm{e}+06$ & 4.3223e-119 & Reject \\
\hline topic493 & 0.00011899 & $5.7351 \mathrm{e}-05$ & $1.84886 \mathrm{e}+06$ & $2.8139 \mathrm{e}-64$ & Reject \\
\hline topic494 & 0.00012311 & 0.00011022 & $2.45172 \mathrm{e}+06$ & $4.487 \mathrm{e}-05$ & Reject \\
\hline topic495 & $8.3791 \mathrm{e}-05$ & $2.4202 \mathrm{e}-06$ & 872329 & 0 & Reject \\
\hline topic496 & 0.00011211 & $6.6619 \mathrm{e}-05$ & $2.07025 \mathrm{e}+06$ & $5.5284 \mathrm{e}-36$ & Reject \\
\hline topic497 & 0.00018398 & 0.00013585 & $1.88336 \mathrm{e}+06$ & $1.9559 \mathrm{e}-62$ & Reject \\
\hline topic498 & $5.6925 \mathrm{e}-05$ & $6.7026 \mathrm{e}-05$ & $2.388 \mathrm{e}+06$ & $3.6097 \mathrm{e}-08$ & Reject \\
\hline topic499 & $3.0212 \mathrm{e}-05$ & $3.169 \mathrm{e}-07$ & $1.9078 \mathrm{e}+06$ & $1.409 \mathrm{e}-18$ & Reject \\
\hline topic500 & 0.00013979 & 0.00010927 & $2.07894 \mathrm{e}+06$ & $6.1744 \mathrm{e}-35$ & Reject \\
\hline topic501 & 0.00012572 & $7.2447 \mathrm{e}-05$ & $1.50259 \mathrm{e}+06$ & $3.5572 \mathrm{e}-139$ & Reject \\
\hline top & $4.2346 \mathrm{e}-05$ & & $1.64861 \mathrm{e}+06$ & $4.0611 \mathrm{e}-106$ & \\
\hline topic503 & $3.7455 \mathrm{e}-05$ & 0.00010038 & $1.71239 \mathrm{e}+06$ & $2.3125 \mathrm{e}-92$ & Reject \\
\hline topic504 & 0.0001179 & 0.00012482 & $2.55434 \mathrm{e}+06$ & 0.0493 & Reject \\
\hline topic505 & 0.00013493 & $8.0734 \mathrm{e}-05$ & $1.76733 \mathrm{e}+06$ & $8.5992 \mathrm{e}-81$ & Reject \\
\hline topic506 & $7.0473 \mathrm{e}-05$ & $8 \mathrm{e}-05$ & $2.1625 \mathrm{e}+06$ & $3.5862 \mathrm{e}-25$ & Reject \\
\hline & & & $2.25598 \mathrm{e}+06$ & -17 & \\
\hline topic508 & 0.00012328 & 0.00014815 & $2.23587 \mathrm{e}+06$ & $1.0372 \mathrm{e}-18$ & Reject \\
\hline topic509 & 0.00010689 & $6.1641 \mathrm{e}-05$ & $1.89344 \mathrm{e}+06$ & $9.1995 \mathrm{e}-59$ & Reject \\
\hline topic510 & 0.00011392 & 0.00010118 & $2.50531 \mathrm{e}+06$ & 0.0035136 & Reject \\
\hline topic511 & 0.00011183 & $7.9983 \mathrm{e}-05$ & $1.81993 \mathrm{e}+06$ & $4.0434 \mathrm{e}-73$ & Reject \\
\hline topic512 & 0.00011387 & $7.4844 \mathrm{e}-05$ & $1.8271 \mathrm{e}+06$ & $1.5179 \mathrm{e}-71$ & Reject \\
\hline topic513 & $1.8566 \mathrm{e}-06$ & $9.1089 \mathrm{e}-06$ & $1.9704 \mathrm{e}+06$ & $1.106 \mathrm{e}-39$ & Reject \\
\hline topic514 & 0.00014905 & 0.0001271 & $2.17128 \mathrm{e}+06$ & $1.6752 \mathrm{e}-24$ & Reject \\
\hline & 0.00010776 & $6.7159 \mathrm{e}-05$ & $1.85533 \mathrm{e}+06$ & $1.9504 \mathrm{e}-66$ & Reject \\
\hline topic516 & $9.6078 \mathrm{e}-05$ & $4.8246 \mathrm{e}-05$ & $1.98843 \mathrm{e}+06$ & $4.1539 \mathrm{e}-46$ & Reject \\
\hline topic517 & 0.0001197 & $7.14 \mathrm{e}-05$ & $1.67778 \mathrm{e}+06$ & $7.6199 \mathrm{e}-100$ & Reject \\
\hline top & 0.00013585 & $9.1634 \mathrm{e}-05$ & $1.65894 \mathrm{e}+06$ & $1.4206 \mathrm{e}-103$ & Reject \\
\hline topic519 & $7.2749 \mathrm{e}-05$ & $5.424 \mathrm{e}-05$ & $2.346 \mathrm{e}+06$ & $2.8456 \mathrm{e}-10$ & Reject \\
\hline & $6.716 \mathrm{e}-05$ & $5.2316 \mathrm{e}-05$ & $2.22775 \mathrm{e}+06$ & $1.9959 \mathrm{e}-18$ & Reject \\
\hline 521 & $7.8941 \mathrm{e}-05$ & $3.4116 \mathrm{e}-05$ & $1.25375 \mathrm{e}+06$ & $7.3094 \mathrm{e}-207$ & Reject \\
\hline topic522 & 0.00013269 & $8.7374 \mathrm{e}-05$ & $1.98928 \mathrm{e}+06$ & $3.9829 \mathrm{e}-46$ & Reject \\
\hline & $2.1721 \mathrm{e}-05$ & $2.7273 \mathrm{e}-05$ & $2.50802 \mathrm{e}+06$ & 0.0052034 & Reject \\
\hline topic524 & $8.5683 \mathrm{e}-05$ & $2.0479 \mathrm{e}-05$ & 917671 & 0 & Reject \\
\hline topic525 & 0.00015583 & 0.00011676 & $2.11982 \mathrm{e}+06$ & $1.4159 \mathrm{e}-29$ & Reject \\
\hline topic526 & 0.00014136 & 0.0001097 & $1.98178 \mathrm{e}+06$ & $1.9672 \mathrm{e}-47$ & Reject \\
\hline topic527 & $6.3248 \mathrm{e}-05$ & $3.451 \mathrm{e}-05$ & $1.9642 \mathrm{e}+06$ & $8.3546 \mathrm{e}-50$ & Reject \\
\hline topic528 & 0.00011568 & 7.5093e-05 & $1.83231 \mathrm{e}+06$ & $5.0932 \mathrm{e}-70$ & Reject \\
\hline topic529 & 0.00010411 & $6.3003 \mathrm{e}-05$ & $2.00624 \mathrm{e}+06$ & $6.8105 \mathrm{e}-43$ & Reject \\
\hline
\end{tabular}


Table A1 - Continued from previous page

\begin{tabular}{|c|c|c|c|c|c|}
\hline Feature & EN median & FL median & u-value & p-value & $\mathbf{H}_{0}$ \\
\hline topic530 & 0.00011384 & $8.2878 \mathrm{e}-05$ & $2.19821 \mathrm{e}+06$ & $6.6204 \mathrm{e}-22$ & Reject \\
\hline topic531 & $3.0013 \mathrm{e}-05$ & $1.3139 \mathrm{e}-05$ & $1.49222 \mathrm{e}+06$ & 6.2163e-142 & Reject \\
\hline topic532 & 0.00010046 & $8.8338 \mathrm{e}-05$ & $2.55595 \mathrm{e}+06$ & 0.050294 & Accept \\
\hline topic533 & 0.00015902 & 0.00014451 & $2.37843 \mathrm{e}+06$ & $1.0773 \mathrm{e}-08$ & Reject \\
\hline topic534 & $9.8619 \mathrm{e}-05$ & $6.1844 \mathrm{e}-05$ & $1.86459 \mathrm{e}+06$ & $3.4042 \mathrm{e}-65$ & Reject \\
\hline topic535 & 0.00014912 & $9.2492 \mathrm{e}-05$ & $2.04923 \mathrm{e}+06$ & $1.0014 \mathrm{e}-37$ & Reject \\
\hline topic536 & 0.00012147 & 0.00013078 & $2.46281 \mathrm{e}+06$ & 0.00011092 & Reject \\
\hline topic537 & 0.00012255 & $9.5324 \mathrm{e}-05$ & $2.24139 \mathrm{e}+06$ & $3.7088 \mathrm{e}-18$ & Reject \\
\hline topic538 & $6.8442 \mathrm{e}-05$ & $4.7957 \mathrm{e}-05$ & $2.16624 \mathrm{e}+06$ & $1.6751 \mathrm{e}-24$ & Reject \\
\hline topic539 & 0.00013597 & $9.4329 \mathrm{e}-05$ & $2.36212 \mathrm{e}+06$ & $1.4427 \mathrm{e}-09$ & Reject \\
\hline topic540 & 0.00012099 & 0.00012531 & $2.55934 \mathrm{e}+06$ & 0.068112 & Accept \\
\hline topic541 & 0.00013243 & $9.1487 \mathrm{e}-05$ & $2.0166 \mathrm{e}+06$ & $1.1179 \mathrm{e}-42$ & Reject \\
\hline topic542 & $7.3195 \mathrm{e}-05$ & $6.1675 \mathrm{e}-05$ & $2.37699 \mathrm{e}+06$ & $8.956 \mathrm{e}-09$ & Reject \\
\hline topic543 & 0.00018998 & 0.0002096 & $2.37598 \mathrm{e}+06$ & $7.8569 \mathrm{e}-09$ & Reject \\
\hline topic544 & 0.00013988 & $7.4477 \mathrm{e}-05$ & $1.75166 \mathrm{e}+06$ & $3.0479 \mathrm{e}-85$ & Reject \\
\hline topic545 & $5.803 \mathrm{e}-05$ & $3.3752 \mathrm{e}-05$ & $2.02952 \mathrm{e}+06$ & $9.8677 \mathrm{e}-41$ & Reject \\
\hline topic546 & $8.5974 \mathrm{e}-05$ & $5.8733 \mathrm{e}-05$ & $1.61507 \mathrm{e}+06$ & $1.5394 \mathrm{e}-113$ & Reject \\
\hline topic547 & 0.0001463 & 0.00011071 & $2.30506 \mathrm{e}+06$ & $3.2836 \mathrm{e}-13$ & Reject \\
\hline topic548 & 0.00013646 & 0.00012 & $2.17578 \mathrm{e}+06$ & $2.3415 \mathrm{e}-24$ & Reject \\
\hline topic549 & $9.0075 \mathrm{e}-05$ & $8.1278 \mathrm{e}-05$ & $2.42727 \mathrm{e}+06$ & $3.2368 \mathrm{e}-06$ & Reject \\
\hline topic550 & 0.00011528 & $6.5838 \mathrm{e}-05$ & $1.92625 \mathrm{e}+06$ & $7.2798 \mathrm{e}-55$ & Reject \\
\hline topic551 & 0.00011142 & 0.00011016 & $2.53944 \mathrm{e}+06$ & 0.035298 & Reject \\
\hline topic552 & 0.00012453 & 0.00013359 & $2.5649 \mathrm{e}+06$ & 0.20269 & Accept \\
\hline topic553 & 0.00012978 & 0.00011071 & $2.24622 \mathrm{e}+06$ & 7.8275e-18 & Reject \\
\hline topic554 & 0.00010564 & $5.9787 \mathrm{e}-05$ & $1.78928 \mathrm{e}+06$ & $2.0036 \mathrm{e}-78$ & Reject \\
\hline topic555 & 0.00013448 & 0.00012758 & $2.48864 \mathrm{e}+06$ & 0.00091446 & Reject \\
\hline topic556 & $4.8508 \mathrm{e}-05$ & $4.6853 \mathrm{e}-07$ & 742866 & 0 & Reject \\
\hline topic557 & 0.00011997 & $9.2367 \mathrm{e}-05$ & $2.10919 \mathrm{e}+06$ & 2.6698e-31 & Reject \\
\hline topic558 & $4.579 \mathrm{e}-05$ & 4.4013e-05 & $2.53291 \mathrm{e}+06$ & 0.015561 & Reject \\
\hline topic559 & $4.8428 \mathrm{e}-05$ & $3.4545 \mathrm{e}-05$ & $2.12528 \mathrm{e}+06$ & $4.4306 \mathrm{e}-28$ & Reject \\
\hline topic560 & $5.4707 \mathrm{e}-05$ & $4.2481 \mathrm{e}-05$ & $2.30103 \mathrm{e}+06$ & $1.4386 \mathrm{e}-13$ & Reject \\
\hline topic561 & $3.7014 \mathrm{e}-05$ & 0.00011693 & $1.61314 \mathrm{e}+06$ & $9.4317 \mathrm{e}-106$ & Reject \\
\hline topic562 & 0.00013917 & $6.5197 \mathrm{e}-05$ & $1.43455 \mathrm{e}+06$ & $8.0796 \mathrm{e}-155$ & Reject \\
\hline topic563 & 0.00013448 & 0.00010043 & $1.84628 \mathrm{e}+06$ & $1.4169 \mathrm{e}-68$ & Reject \\
\hline topic564 & $8.7262 \mathrm{e}-05$ & $6.2819 \mathrm{e}-05$ & $2.35879 \mathrm{e}+06$ & $1.0662 \mathrm{e}-09$ & Reject \\
\hline topic565 & 0.00022166 & 0.00024356 & $2.46102 \mathrm{e}+06$ & $9.4741 \mathrm{e}-05$ & Reject \\
\hline topic566 & 0.00013862 & 9.4987 e-05 & $1.98844 \mathrm{e}+06$ & $1.6745 \mathrm{e}-46$ & Reject \\
\hline topic567 & $1.8502 \mathrm{e}-07$ & $2.1673 \mathrm{e}-07$ & $2.39127 \mathrm{e}+06$ & 0.00017918 & Reject \\
\hline topic568 & 0.00013751 & $6.0578 \mathrm{e}-05$ & $1.42362 \mathrm{e}+06$ & $1.7497 \mathrm{e}-159$ & Reject \\
\hline topic569 & $9.8167 \mathrm{e}-05$ & $9.9942 \mathrm{e}-05$ & $2.62322 \mathrm{e}+06$ & 0.4434 & Accept \\
\hline topic570 & 0.00013954 & 0.00016222 & $2.35853 \mathrm{e}+06$ & $7.5824 \mathrm{e}-10$ & Reject \\
\hline topic571 & $4.954 \mathrm{e}-05$ & $2.483 \mathrm{e}-05$ & $1.84187 \mathrm{e}+06$ & $1.565 \mathrm{e}-68$ & Reject \\
\hline
\end{tabular}


Table A1 - Continued from previous page

\begin{tabular}{|c|c|c|c|c|c|}
\hline Feature & EN median & FL median & u-value & p-value & $\mathbf{H}_{0}$ \\
\hline topic572 & $2.1092 \mathrm{e}-05$ & $9.2274 \mathrm{e}-06$ & $1.68513 \mathrm{e}+06$ & $1.6125 \mathrm{e}-98$ & Reject \\
\hline topic573 & 0.000131 & 0.00010727 & $2.13305 \mathrm{e}+06$ & 8.8933e-29 & Reject \\
\hline topic574 & 0.00013575 & 0.00010384 & $2.16593 \mathrm{e}+06$ & $3.9347 \mathrm{e}-25$ & Reject \\
\hline topic575 & 0.00013598 & $6.7242 \mathrm{e}-05$ & $1.65463 \mathrm{e}+06$ & $7.5828 \mathrm{e}-105$ & Reject \\
\hline topic576 & $2.3581 \mathrm{e}-05$ & $7.3624 \mathrm{e}-06$ & $1.56786 \mathrm{e}+06$ & $1.0483 \mathrm{e}-121$ & Reject \\
\hline topic577 & 0.00017258 & 0.00019603 & $2.42632 \mathrm{e}+06$ & $2.9286 \mathrm{e}-06$ & Reject \\
\hline topic578 & $5.946 \mathrm{e}-05$ & $5.2792 \mathrm{e}-05$ & $2.41364 \mathrm{e}+06$ & 7.3915e-07 & Reject \\
\hline topic579 & 0.00016555 & 0.00016161 & $2.56364 \mathrm{e}+06$ & 0.074336 & Accept \\
\hline topic580 & 0.00013316 & 0.00010413 & $2.08794 \mathrm{e}+06$ & $9.4875 \mathrm{e}-34$ & Reject \\
\hline topic581 & 0.00013039 & $5.0199 \mathrm{e}-05$ & $1.06118 \mathrm{e}+06$ & $4.2322 \mathrm{e}-266$ & Reject \\
\hline topic582 & $7.0865 \mathrm{e}-06$ & $7.5194 \mathrm{e}-06$ & $2.5695 \mathrm{e}+06$ & 0.15083 & Accept \\
\hline topic583 & $8.4373 \mathrm{e}-05$ & $2.4162 \mathrm{e}-05$ & $1.07281 \mathrm{e}+06$ & $1.7278 \mathrm{e}-263$ & Reject \\
\hline topic584 & $7.0808 \mathrm{e}-05$ & $6.0416 \mathrm{e}-05$ & $2.21161 \mathrm{e}+06$ & $5.9505 \mathrm{e}-21$ & Reject \\
\hline topic585 & $6.531 \mathrm{e}-05$ & $7.2387 \mathrm{e}-05$ & $2.44222 \mathrm{e}+06$ & $1.6486 \mathrm{e}-05$ & Reject \\
\hline topic586 & $9.82 \mathrm{e}-05$ & 0.00011892 & $2.27333 \mathrm{e}+06$ & $9.9692 \mathrm{e}-16$ & Reject \\
\hline topic587 & 0.00014262 & 0.00010749 & $2.19594 \mathrm{e}+06$ & $4.1328 \mathrm{e}-22$ & Reject \\
\hline topic588 & $7.639 \mathrm{e}-05$ & $3.173 \mathrm{e}-05$ & $1.50423 \mathrm{e}+06$ & $1.3332 \mathrm{e}-137$ & Reject \\
\hline topic589 & $3.9799 \mathrm{e}-05$ & $1.8925 \mathrm{e}-05$ & $1.77864 \mathrm{e}+06$ & $3.3427 \mathrm{e}-80$ & Reject \\
\hline topic590 & 0.00013294 & 0.00013571 & $2.56254 \mathrm{e}+06$ & 0.070951 & Accept \\
\hline topic591 & 0.00011572 & 0.00012886 & $2.54288 \mathrm{e}+06$ & 0.037821 & Reject \\
\hline topic592 & $5.2591 \mathrm{e}-05$ & $3.0174 \mathrm{e}-05$ & $1.81427 \mathrm{e}+06$ & $4.088 \mathrm{e}-74$ & Reject \\
\hline topic593 & $4.6787 \mathrm{e}-05$ & $3.3983 \mathrm{e}-05$ & $1.63463 \mathrm{e}+06$ & $4.1863 \mathrm{e}-109$ & Reject \\
\hline topic594 & $4.5591 \mathrm{e}-05$ & $1.8806 \mathrm{e}-05$ & $1.54324 \mathrm{e}+06$ & $1.0224 \mathrm{e}-128$ & Reject \\
\hline topic595 & 0.00019833 & 0.00017939 & $2.308 \mathrm{e}+06$ & $5.2963 \mathrm{e}-13$ & Reject \\
\hline topic596 & $3.1828 \mathrm{e}-05$ & $1.8224 \mathrm{e}-05$ & $2.01513 \mathrm{e}+06$ & $5.2604 \mathrm{e}-43$ & Reject \\
\hline topic597 & 0.00010324 & $6.9691 \mathrm{e}-05$ & $1.98071 \mathrm{e}+06$ & $2.5526 \mathrm{e}-47$ & Reject \\
\hline topic598 & 0.00012926 & 0.0001386 & $2.52256 \mathrm{e}+06$ & 0.0090777 & Reject \\
\hline topic599 & 0.00012516 & 0.00015981 & $2.21172 \mathrm{e}+06$ & $6.087 \mathrm{e}-21$ & Reject \\
\hline topic600 & 0.00012259 & $5.5832 \mathrm{e}-05$ & $1.64043 \mathrm{e}+06$ & $9.0712 \mathrm{e}-107$ & Reject \\
\hline topic601 & $4.4753 \mathrm{e}-05$ & $3.1062 \mathrm{e}-05$ & $2.09615 \mathrm{e}+06$ & $1.4465 \mathrm{e}-32$ & Reject \\
\hline topic602 & $7.1526 \mathrm{e}-05$ & $5.5091 \mathrm{e}-05$ & $2.03257 \mathrm{e}+06$ & $1.0308 \mathrm{e}-40$ & Reject \\
\hline topic603 & 0.00010822 & $6.4819 \mathrm{e}-05$ & $1.86505 \mathrm{e}+06$ & $5.7884 \mathrm{e}-65$ & Reject \\
\hline topic604 & 0.00010539 & $9.8079 \mathrm{e}-05$ & $2.52274 \mathrm{e}+06$ & 0.0092298 & Reject \\
\hline topic605 & 0.0001299 & 0.00013542 & $2.58198 \mathrm{e}+06$ & 0.14417 & Accept \\
\hline topic606 & $4.4407 \mathrm{e}-05$ & $2.4197 \mathrm{e}-05$ & $2.2609 \mathrm{e}+06$ & $1.2523 \mathrm{e}-16$ & Reject \\
\hline topic607 & 0.00012675 & 0.00012296 & $2.55624 \mathrm{e}+06$ & 0.050977 & Accept \\
\hline topic608 & $7.0559 \mathrm{e}-05$ & $9.7305 \mathrm{e}-05$ & $2.27827 \mathrm{e}+06$ & $2.4053 \mathrm{e}-15$ & Reject \\
\hline topic609 & $4.1323 \mathrm{e}-05$ & $2.3423 \mathrm{e}-05$ & $1.87183 \mathrm{e}+06$ & $5.2772 \mathrm{e}-64$ & Reject \\
\hline topic610 & $9.9941 \mathrm{e}-05$ & $6.8781 \mathrm{e}-05$ & $2.04888 \mathrm{e}+06$ & $1.6789 \mathrm{e}-38$ & Reject \\
\hline topic611 & $5.6211 \mathrm{e}-05$ & $4.2946 \mathrm{e}-07$ & 820053 & 0 & Reject \\
\hline topic612 & 0.00017918 & $9.6603 \mathrm{e}-05$ & $1.58806 \mathrm{e}+06$ & $3.6725 \mathrm{e}-119$ & Reject \\
\hline topic613 & $7.8513 \mathrm{e}-05$ & $4.6181 \mathrm{e}-05$ & $1.75771 \mathrm{e}+06$ & $8.9682 \mathrm{e}-84$ & Reject \\
\hline
\end{tabular}


Table A1 - Continued from previous page

\begin{tabular}{|c|c|c|c|c|c|}
\hline Feature & EN median & FL median & u-value & p-value & $\mathbf{H}_{0}$ \\
\hline topic614 & $6.7295 \mathrm{e}-05$ & $3.6763 \mathrm{e}-05$ & $1.74159 \mathrm{e}+06$ & $5.4361 \mathrm{e}-87$ & Reject \\
\hline topic615 & $7.4699 \mathrm{e}-05$ & $3.1677 \mathrm{e}-05$ & $1.5067 \mathrm{e}+06$ & $5.6025 \mathrm{e}-138$ & Reject \\
\hline topic616 & 0.00019657 & 0.00018601 & $2.49432 \mathrm{e}+06$ & 0.001385 & Reject \\
\hline topic617 & $8.5067 \mathrm{e}-08$ & $8.0299 \mathrm{e}-08$ & $2.13989 \mathrm{e}+06$ & 0.024365 & Reject \\
\hline topic618 & $4.6597 \mathrm{e}-05$ & $1.2679 \mathrm{e}-05$ & $1.17544 \mathrm{e}+06$ & $1.3445 \mathrm{e}-230$ & Reject \\
\hline topic619 & $9.8512 \mathrm{e}-06$ & 7.7095e-06 & $2.41708 \mathrm{e}+06$ & $2.4888 \mathrm{e}-06$ & Reject \\
\hline topic620 & 0.00012181 & $5.4699 \mathrm{e}-05$ & $1.54576 \mathrm{e}+06$ & $1.6895 \mathrm{e}-128$ & Reject \\
\hline topic621 & $8.4809 \mathrm{e}-05$ & $3.878 \mathrm{e}-05$ & $2.1538 \mathrm{e}+06$ & $2.2832 \mathrm{e}-26$ & Reject \\
\hline topic622 & 0.00014406 & 0.00012951 & $2.459 \mathrm{e}+06$ & $9.6144 \mathrm{e}-05$ & Reject \\
\hline topic623 & 0.00012529 & 0.00010002 & $2.11922 \mathrm{e}+06$ & $3.5352 \mathrm{e}-30$ & Reject \\
\hline topic624 & $1.2197 \mathrm{e}-05$ & $2.4365 \mathrm{e}-05$ & $2.06517 \mathrm{e}+06$ & $4.3415 \mathrm{e}-30$ & Reject \\
\hline topic625 & 0.00011624 & $8.4464 \mathrm{e}-05$ & $2.1594 \mathrm{e}+06$ & $5.261 \mathrm{e}-26$ & Reject \\
\hline topic626 & 0.00015959 & 0.00011056 & $2.07283 \mathrm{e}+06$ & $1.0148 \mathrm{e}-34$ & Reject \\
\hline topic627 & $9.647 \mathrm{e}-05$ & $7.0272 \mathrm{e}-05$ & $2.17688 \mathrm{e}+06$ & $3.0021 \mathrm{e}-24$ & Reject \\
\hline topic628 & $4.2137 \mathrm{e}-05$ & $4.2021 \mathrm{e}-05$ & $2.59289 \mathrm{e}+06$ & 0.21349 & Accept \\
\hline topic629 & $4.1918 \mathrm{e}-05$ & $3.4557 \mathrm{e}-05$ & $2.40768 \mathrm{e}+06$ & $4.8118 \mathrm{e}-07$ & Reject \\
\hline topic630 & 0.00010419 & $9.5957 \mathrm{e}-05$ & $2.48949 \mathrm{e}+06$ & 0.0012432 & Reject \\
\hline topic631 & 0.00013817 & $8.5745 \mathrm{e}-05$ & $1.81264 \mathrm{e}+06$ & $2.7209 \mathrm{e}-73$ & Reject \\
\hline topic632 & 0.0001319 & 0.00012365 & $2.6231 \mathrm{e}+06$ & 0.49356 & Accept \\
\hline topic633 & $5.5737 \mathrm{e}-05$ & $4.1725 \mathrm{e}-05$ & $2.05452 \mathrm{e}+06$ & $8.5256 \mathrm{e}-38$ & Reject \\
\hline topic634 & $6.091 \mathrm{e}-05$ & $5.6872 \mathrm{e}-05$ & $2.48454 \mathrm{e}+06$ & 0.00066799 & Reject \\
\hline topic635 & 0.00010244 & $5.0576 \mathrm{e}-05$ & $1.7286 \mathrm{e}+06$ & $3.4845 \mathrm{e}-89$ & Reject \\
\hline topic636 & 0.00015256 & 0.00024484 & $1.74738 \mathrm{e}+06$ & $2.1201 \mathrm{e}-86$ & Reject \\
\hline topic637 & $5.8948 \mathrm{e}-05$ & $2.6712 \mathrm{e}-05$ & $1.52805 \mathrm{e}+06$ & 7.1771e-133 & Reject \\
\hline topic638 & 0.00012912 & 0.00015096 & $2.2634 \mathrm{e}+06$ & $2.9539 \mathrm{e}-16$ & Reject \\
\hline topic639 & 0.00020363 & 0.00011617 & $2.1693 \mathrm{e}+06$ & $6.7159 \mathrm{e}-24$ & Reject \\
\hline topic640 & $9.8545 \mathrm{e}-05$ & $6.3547 \mathrm{e}-05$ & $1.94986 \mathrm{e}+06$ & $9.5245 \mathrm{e}-52$ & Reject \\
\hline topic641 & 0.00018582 & 0.00015897 & $2.28372 \mathrm{e}+06$ & $6.2782 \mathrm{e}-15$ & Reject \\
\hline topic642 & 0.00013114 & $8.1567 \mathrm{e}-05$ & $1.87291 \mathrm{e}+06$ & $5.5685 \mathrm{e}-64$ & Reject \\
\hline topic643 & 0.00010747 & $7.4387 \mathrm{e}-05$ & $2.20051 \mathrm{e}+06$ & $2.1727 \mathrm{e}-21$ & Reject \\
\hline topic644 & 0.00013355 & 0.00011479 & $2.04364 \mathrm{e}+06$ & $2.721 \mathrm{e}-39$ & Reject \\
\hline topic645 & $3.0016 \mathrm{e}-05$ & $2.1617 \mathrm{e}-05$ & $2.1105 \mathrm{e}+06$ & $8.3002 \mathrm{e}-31$ & Reject \\
\hline topic646 & 0.00012054 & 0.00012294 & $2.60947 \mathrm{e}+06$ & 0.3651 & Accept \\
\hline topic647 & $4.4318 \mathrm{e}-05$ & $2.3331 \mathrm{e}-05$ & $1.92972 \mathrm{e}+06$ & $3.399 \mathrm{e}-54$ & Reject \\
\hline topic648 & 0.00010174 & 0.00014092 & $2.13037 \mathrm{e}+06$ & $5.8933 \mathrm{e}-29$ & Reject \\
\hline topic649 & $4.8324 \mathrm{e}-05$ & $5.0336 \mathrm{e}-05$ & $2.58174 \mathrm{e}+06$ & 0.14901 & Accept \\
\hline topic650 & 0.00010449 & $9.7777 \mathrm{e}-05$ & $2.49302 \mathrm{e}+06$ & 0.0016194 & Reject \\
\hline topic651 & 0.00010051 & $6.3849 \mathrm{e}-05$ & $1.82587 \mathrm{e}+06$ & $8.9754 \mathrm{e}-72$ & Reject \\
\hline topic652 & $1.029 \mathrm{e}-05$ & $3.0683 \mathrm{e}-05$ & $1.69098 \mathrm{e}+06$ & $3.414 \mathrm{e}-79$ & Reject \\
\hline topic653 & $1.1165 \mathrm{e}-05$ & $1.8374 \mathrm{e}-05$ & $2.21486 \mathrm{e}+06$ & $9.3077 \mathrm{e}-20$ & Reject \\
\hline topic654 & $9.1006 \mathrm{e}-05$ & $6.1886 \mathrm{e}-05$ & $2.05237 \mathrm{e}+06$ & $3.4423 \mathrm{e}-38$ & Reject \\
\hline topic655 & 0.00014061 & 0.00011083 & $2.05916 \mathrm{e}+06$ & $2.4169 \mathrm{e}-37$ & Reject \\
\hline
\end{tabular}


Table A1 - Continued from previous page

\begin{tabular}{|c|c|c|c|c|c|}
\hline Feature & EN median & FL median & u-value & p-value & $\mathrm{H}_{\mathbf{0}}$ \\
\hline topic656 & $5.6344 \mathrm{e}-05$ & $5.2989 \mathrm{e}-05$ & $2.61264 \mathrm{e}+06$ & 0.3717 & Accept \\
\hline topic657 & 0.00012264 & $6.8488 \mathrm{e}-05$ & $1.57107 \mathrm{e}+06$ & $1.8642 \mathrm{e}-122$ & Reject \\
\hline topic658 & $8.3359 \mathrm{e}-05$ & 0.0001118 & $2.19257 \mathrm{e}+06$ & $1.2552 \mathrm{e}-22$ & Reject \\
\hline topic659 & be-05 & $7.687 \mathrm{e}-05$ & $2.37795 \mathrm{e}+06$ & $1.1691 \mathrm{e}-08$ & Reject \\
\hline topic660 & $4.1458 \mathrm{e}-05$ & $1.3341 \mathrm{e}-05$ & $1.13562 \mathrm{e}+06$ & $1.2616 \mathrm{e}-242$ & Reject \\
\hline topic661 & $5.8267 \mathrm{e}-05$ & $5.079 \mathrm{e}-05$ & $2.38767 \mathrm{e}+06$ & $5.2389 \mathrm{e}-08$ & Reject \\
\hline topic662 & 0.00014862 & 0.00017325 & $2.42847 \mathrm{e}+06$ & $4.0875 \mathrm{e}-06$ & Reject \\
\hline topic663 & 0.00011974 & $5.3404 \mathrm{e}-05$ & $1.51956 \mathrm{e}+06$ & $2.6344 \mathrm{e}-134$ & Reject \\
\hline topic664 & 0.0001189 & 0.00010559 & $2.41063 \mathrm{e}+06$ & $5.9838 \mathrm{e}-07$ & Reject \\
\hline topic665 & 0.00015434 & 0.00011028 & $2.09968 \mathrm{e}+06$ & $4.7103 \mathrm{e}-32$ & Reject \\
\hline topic666 & 0.00013664 & 0.00011925 & $2.17429 \mathrm{e}+06$ & $1.6662 \mathrm{e}-24$ & Reject \\
\hline topic667 & $6.3759 \mathrm{e}-05$ & $9.6473 \mathrm{e}-05$ & $2.16863 \mathrm{e}+06$ & $4.534 \mathrm{e}-25$ & Reject \\
\hline topic668 & 0.00011286 & $8.0223 \mathrm{e}-05$ & $1.93084 \mathrm{e}+06$ & $5.1784 \mathrm{e}-54$ & Reject \\
\hline topic669 & $3.6789 \mathrm{e}-05$ & 3.72 & $2.61156 \mathrm{e}+06$ & 0.35272 & Accept \\
\hline top & 4.82 & & & $221 \mathrm{e}-305$ & Reject \\
\hline topic671 & $2.8889 \mathrm{e}-05$ & $2.466 \mathrm{e}-05$ & $2.58420 \mathrm{e}+06$ & 0.16869 & Accept \\
\hline 672 & 0.00012567 & $9.1878 \mathrm{e}-05$ & $1.99224 \mathrm{e}+06$ & $1.0496 \mathrm{e}-45$ & Reject \\
\hline topic673 & $8.4792 \mathrm{e}-05$ & $8.8186 \mathrm{e}-05$ & $2.60205 \mathrm{e}+06$ & 0.2873 & Accept \\
\hline 674 & e-05 & $2.4129 \mathrm{e}-05$ & $1.59463 \mathrm{e}+06$ & $4.5636 \mathrm{e}-118$ & Reject \\
\hline & & & $324 \mathrm{e}+06$ & $.2156 \mathrm{e}-54$ & \\
\hline topic676 & 0.00013557 & 0.00014492 & $2.58936 \mathrm{e}+06$ & 0.2621 & Accept \\
\hline topic677 & 0.00013452 & 01174 & $2.34097 \mathrm{e}+06$ & $232 \mathrm{e}-10$ & Reject \\
\hline topic678 & $857 \mathrm{e}-05$ & $3.961 \mathrm{e}-05$ & $1.83718 \mathrm{e}+06$ & $.7303 \mathrm{e}-70$ & Reject \\
\hline topic679 & $5.9031 \mathrm{e}-05$ & $3.9549 \mathrm{e}-05$ & $1.69337 \mathrm{e}+06$ & $5.0542 \mathrm{e}-97$ & Reject \\
\hline topic680 & 0.00011868 & $6.2601 \mathrm{e}-05$ & $1.42628 \mathrm{e}+06$ & $2.1321 \mathrm{e}-158$ & Reject \\
\hline topic681 & $8035 \mathrm{e}-06$ & $3.7795 \mathrm{e}-07$ & $2.06103 \mathrm{e}+06$ & $.4067 \mathrm{e}-20$ & Reject \\
\hline topic682 & 0.00015909 & 0.00013492 & $2.24704 \mathrm{e}+06$ & $1.3564 \mathrm{e}-17$ & Reject \\
\hline & 00012688 & $9.7721 \mathrm{e}-05$ & $2.29134 \mathrm{e}+06$ & $6.8433 \mathrm{e}-14$ & Reject \\
\hline 684 & 0.00011501 & 0.00012811 & $2.42722 \mathrm{e}+06$ & $3.6226 \mathrm{e}-06$ & Reject \\
\hline 685 & 00010707 & -05 & $1.68560 \mathrm{e}+06$ & $217 \mathrm{e}-97$ & Reject \\
\hline topic686 & $1.5022 \mathrm{e}-05$ & $2.7578 \mathrm{e}-05$ & $1.97208 \mathrm{e}+06$ & $2.0659 \mathrm{e}-48$ & Reject \\
\hline topic687 & $9.847 \mathrm{e}-05$ & $7.4221 \mathrm{e}-05$ & $1.90067 \mathrm{e}+06$ & $1.6132 \mathrm{e}-59$ & Reject \\
\hline & 0.000127 & 5.67 & $1.70035 \mathrm{e}+06$ & $1.6298 \mathrm{e}-69$ & Reject \\
\hline top & .00011078 & $6.2111 \mathrm{e}-05$ & $1.74726 \mathrm{e}+06$ & $4.4429 \mathrm{e}-86$ & Reject \\
\hline topic690 & 0.00011579 & $9.7307 \mathrm{e}-05$ & $2.30594 \mathrm{e}+06$ & $2.7007 \mathrm{e}-13$ & Reject \\
\hline & $7.7907 \mathrm{e}-05$ & $9.8673 \mathrm{e}-05$ & $2.28824 \mathrm{e}+06$ & $1.3774 \mathrm{e}-14$ & Reject \\
\hline topic692 & 0.00010958 & $8.9654 \mathrm{e}-05$ & $2.28532 \mathrm{e}+06$ & $1.0016 \mathrm{e}-14$ & Reject \\
\hline topic693 & $2.6291 \mathrm{e}-05$ & $1.4486 \mathrm{e}-05$ & $2.00763 \mathrm{e}+06$ & $7.0576 \mathrm{e}-44$ & Reject \\
\hline & $8.907 \mathrm{e}-05$ & $8.1376 \mathrm{e}-05$ & $2.34316 \mathrm{e}+06$ & $8.5541 \mathrm{e}-11$ & Reject \\
\hline topic695 & $1.1251 \mathrm{e}-05$ & $5.1224 \mathrm{e}-06$ & $2.02373 \mathrm{e}+06$ & $9.5238 \mathrm{e}-40$ & Reject \\
\hline topic696 & $5.7032 \mathrm{e}-05$ & $4.9359 \mathrm{e}-05$ & $2.36994 \mathrm{e}+06$ & 4.0661e-09 & Reject \\
\hline topic697 & 0.00016609 & 0.0001281 & $1.90178 \mathrm{e}+06$ & $1.7048 \mathrm{e}-59$ & Reject \\
\hline
\end{tabular}


Table A1 - Continued from previous page

\begin{tabular}{|c|c|c|c|c|c|}
\hline Feature & EN median & FL median & u-value & p-value & $\mathbf{H}_{0}$ \\
\hline topic698 & 0.00011947 & $5.2011 \mathrm{e}-05$ & $1.2966 \mathrm{e}+06$ & $1.847 \mathrm{e}-193$ & Reject \\
\hline topic699 & $7.4711 \mathrm{e}-05$ & $3.6444 \mathrm{e}-05$ & $2.16033 \mathrm{e}+06$ & $1.4272 \mathrm{e}-24$ & Reject \\
\hline topic700 & $6.5192 \mathrm{e}-05$ & $4.5381 \mathrm{e}-05$ & $2.09444 \mathrm{e}+06$ & $5.4587 \mathrm{e}-33$ & Reject \\
\hline topic701 & $4.1971 \mathrm{e}-05$ & $2.5969 \mathrm{e}-05$ & $1.64102 \mathrm{e}+06$ & $1.441 \mathrm{e}-107$ & Reject \\
\hline topic702 & $9.0406 \mathrm{e}-05$ & $3.3012 \mathrm{e}-05$ & $1.52684 \mathrm{e}+06$ & $2.45 \mathrm{e}-133$ & Reject \\
\hline topic703 & 0.00010865 & 0.00011072 & $2.56461 \mathrm{e}+06$ & 0.081282 & Accept \\
\hline topic704 & $8.9491 \mathrm{e}-05$ & $6.7939 \mathrm{e}-05$ & $2.27003 \mathrm{e}+06$ & $9.6767 \mathrm{e}-16$ & Reject \\
\hline topic705 & 0.00013349 & 0.00011402 & $2.42465 \mathrm{e}+06$ & $4.8925 \mathrm{e}-06$ & Reject \\
\hline topic706 & $5.7095 \mathrm{e}-05$ & $3.3787 \mathrm{e}-05$ & $1.80241 \mathrm{e}+06$ & $3.1948 \mathrm{e}-76$ & Reject \\
\hline topic707 & 0.00011652 & $3.6838 \mathrm{e}-05$ & $1.69711 \mathrm{e}+06$ & $1.6004 \mathrm{e}-94$ & Reject \\
\hline topic708 & 0.00013355 & $8.1853 \mathrm{e}-05$ & $1.92394 \mathrm{e}+06$ & $2.548 \mathrm{e}-55$ & Reject \\
\hline topic709 & 0.00013165 & 0.00018988 & $1.869 \mathrm{e}+06$ & $8.8828 \mathrm{e}-65$ & Reject \\
\hline topic710 & 0.00014064 & 0.00010688 & $1.94583 \mathrm{e}+06$ & $2.4961 \mathrm{e}-52$ & Reject \\
\hline topic711 & 0.00011778 & $9.2553 \mathrm{e}-05$ & $2.12271 \mathrm{e}+06$ & $1.0875 \mathrm{e}-29$ & Reject \\
\hline topic712 & 0.00015307 & $9.6461 \mathrm{e}-05$ & $1.88936 \mathrm{e}+06$ & $1.4231 \mathrm{e}-60$ & Reject \\
\hline topic713 & $9.6113 \mathrm{e}-05$ & $7.6523 \mathrm{e}-05$ & $2.41368 \mathrm{e}+06$ & $1.5903 \mathrm{e}-05$ & Reject \\
\hline topic714 & 0.00019847 & 0.00019903 & $2.56043 \mathrm{e}+06$ & 0.067857 & Accept \\
\hline topic715 & 0.00014264 & 0.00010063 & $2.06714 \mathrm{e}+06$ & $4.0728 \mathrm{e}-36$ & Reject \\
\hline topic716 & $8.8873 \mathrm{e}-05$ & $8.0222 \mathrm{e}-05$ & $2.45395 \mathrm{e}+06$ & $6.1688 \mathrm{e}-05$ & Reject \\
\hline topic717 & 0.00022123 & 0.00017517 & $1.91015 \mathrm{e}+06$ & $3.4946 \mathrm{e}-58$ & Reject \\
\hline topic718 & $8.8405 \mathrm{e}-05$ & $4.6059 \mathrm{e}-05$ & $1.41414 \mathrm{e}+06$ & $9.3247 \mathrm{e}-162$ & Reject \\
\hline topic719 & $3.8286 \mathrm{e}-05$ & $2.5677 \mathrm{e}-05$ & $2.06741 \mathrm{e}+06$ & $4.2822 \mathrm{e}-36$ & Reject \\
\hline topic720 & $8.9804 \mathrm{e}-05$ & $5.1576 \mathrm{e}-05$ & $1.46341 \mathrm{e}+06$ & $6.3618 \mathrm{e}-148$ & Reject \\
\hline topic721 & 0.00011993 & $9.4582 \mathrm{e}-05$ & $2.1613 \mathrm{e}+06$ & $1.0483 \mathrm{e}-25$ & Reject \\
\hline topic722 & 0.00012858 & $8.9867 \mathrm{e}-05$ & $1.97678 \mathrm{e}+06$ & $5.3415 \mathrm{e}-48$ & Reject \\
\hline topic723 & 0.00018479 & 0.00019916 & $2.48184 \mathrm{e}+06$ & 0.00049419 & Reject \\
\hline topic724 & $6.9616 \mathrm{e}-05$ & $8.2506 \mathrm{e}-06$ & 971226 & $4.6142 \mathrm{e}-298$ & Reject \\
\hline topic725 & 0.00011672 & $8.5865 \mathrm{e}-05$ & $2.03452 \mathrm{e}+06$ & $3.2454 \mathrm{e}-40$ & Reject \\
\hline topic726 & 0.00012661 & 0.00010774 & $2.13759 \mathrm{e}+06$ & $2.7355 \mathrm{e}-28$ & Reject \\
\hline topic727 & $9.0029 \mathrm{e}-05$ & $4.6059 \mathrm{e}-05$ & $1.40525 \mathrm{e}+06$ & $2.8792 \mathrm{e}-163$ & Reject \\
\hline topic728 & 0.00013305 & 0.0001019 & $2.17142 \mathrm{e}+06$ & $1.0954 \mathrm{e}-24$ & Reject \\
\hline topic729 & 0.00017089 & 0.00013537 & $2.27331 \mathrm{e}+06$ & $1.2055 \mathrm{e}-15$ & Reject \\
\hline topic730 & 0.00010274 & $6.7266 \mathrm{e}-05$ & $1.88754 \mathrm{e}+06$ & $1.8031 \mathrm{e}-61$ & Reject \\
\hline topic731 & 0.00014009 & 0.00011724 & $2.34269 \mathrm{e}+06$ & $9.3832 \mathrm{e}-11$ & Reject \\
\hline topic732 & 0.00012604 & 0.00013309 & $2.51944 \mathrm{e}+06$ & 0.007028 & Reject \\
\hline topic733 & 0.00013009 & 0.00011436 & $2.40886 \mathrm{e}+06$ & $4.8471 \mathrm{e}-07$ & Reject \\
\hline topic734 & $9.7007 \mathrm{e}-05$ & $3.8698 \mathrm{e}-05$ & $1.34264 \mathrm{e}+06$ & $4.4332 \mathrm{e}-181$ & Reject \\
\hline topic735 & $5.0789 \mathrm{e}-05$ & $3.4788 \mathrm{e}-05$ & $2.15774 \mathrm{e}+06$ & $1.8845 \mathrm{e}-25$ & Reject \\
\hline topic736 & $9.4611 \mathrm{e}-05$ & $6.2569 \mathrm{e}-05$ & $2.22784 \mathrm{e}+06$ & 2.5421e-19 & Reject \\
\hline topic737 & 0.0001122 & $8.0861 \mathrm{e}-05$ & $2.13918 \mathrm{e}+06$ & $6.7073 \mathrm{e}-28$ & Reject \\
\hline topic738 & 0.00015159 & 0.00011638 & $2.13199 \mathrm{e}+06$ & $1.4389 \mathrm{e}-28$ & Reject \\
\hline topic739 & 0.00012461 & $8.6054 \mathrm{e}-05$ & $2.04019 \mathrm{e}+06$ & 4.1801e-39 & Reject \\
\hline
\end{tabular}


Table A1 - Continued from previous page

\begin{tabular}{|c|c|c|c|c|c|}
\hline Feature & EN median & FL median & u-value & p-value & $\mathbf{H}_{0}$ \\
\hline topic740 & 0.00012209 & $4.9325 \mathrm{e}-05$ & $1.65404 \mathrm{e}+06$ & $1.1619 \mathrm{e}-102$ & Reject \\
\hline topic741 & $4.3071 \mathrm{e}-05$ & $8.2321 \mathrm{e}-06$ & 976033 & $6.0022 \mathrm{e}-294$ & Reject \\
\hline topic742 & 0.00010366 & $6.312 \mathrm{e}-05$ & $2.0093 \mathrm{e}+06$ & $3.8072 \mathrm{e}-43$ & Reject \\
\hline topic743 & 0.00013925 & $9.9173 \mathrm{e}-05$ & $2.06681 \mathrm{e}+06$ & $1.8689 \mathrm{e}-35$ & Reject \\
\hline topic744 & $1.9621 \mathrm{e}-05$ & $2.2911 \mathrm{e}-05$ & $2.41834 \mathrm{e}+06$ & $1.7944 \mathrm{e}-06$ & Reject \\
\hline topic745 & 0.00010325 & $8.4023 \mathrm{e}-05$ & $2.3932 \mathrm{e}+06$ & $7.7279 \mathrm{e}-08$ & Reject \\
\hline topic746 & 0.00018037 & 0.000191 & $2.49785 \mathrm{e}+06$ & 0.0016563 & Reject \\
\hline topic747 & 0.00016417 & 0.00016074 & $2.61477 \mathrm{e}+06$ & 0.41901 & Accept \\
\hline topic748 & 0.00013253 & 0.00011342 & $2.23413 \mathrm{e}+06$ & $5.9558 \mathrm{e}-19$ & Reject \\
\hline topic749 & $2.7091 \mathrm{e}-07$ & $2.8766 \mathrm{e}-07$ & $2.51157 \mathrm{e}+06$ & 0.076391 & Accept \\
\hline topic750 & 0.00011258 & $7.9348 \mathrm{e}-05$ & $1.82424 \mathrm{e}+06$ & $4.8324 \mathrm{e}-72$ & Reject \\
\hline topic751 & 0.00013913 & $7.9256 \mathrm{e}-05$ & $1.57009 \mathrm{e}+06$ & $1.2653 \mathrm{e}-123$ & Reject \\
\hline topic752 & $9.6819 \mathrm{e}-05$ & $9.6168 \mathrm{e}-05$ & $2.5747 \mathrm{e}+06$ & 0.11047 & Accept \\
\hline topic753 & 0.0001253 & $7.8742 \mathrm{e}-05$ & $2.07274 \mathrm{e}+06$ & $2.4512 \mathrm{e}-35$ & Reject \\
\hline topic754 & $6.2987 \mathrm{e}-06$ & $5.8262 \mathrm{e}-06$ & $2.36292 \mathrm{e}+06$ & 0.0081378 & Reject \\
\hline topic755 & $9.7051 \mathrm{e}-05$ & $6.2277 \mathrm{e}-05$ & $2.01142 \mathrm{e}+06$ & $4.1943 \mathrm{e}-43$ & Reject \\
\hline topic756 & $4.6907 \mathrm{e}-05$ & $3.5452 \mathrm{e}-05$ & $2.19235 \mathrm{e}+06$ & $8.9816 \mathrm{e}-22$ & Reject \\
\hline topic757 & $2.186 \mathrm{e}-05$ & $7.6512 \mathrm{e}-05$ & $1.7471 \mathrm{e}+06$ & $3.5436 \mathrm{e}-79$ & Reject \\
\hline topic758 & 0.00010542 & $8.0767 \mathrm{e}-05$ & $1.98277 \mathrm{e}+06$ & $1.9766 \mathrm{e}-47$ & Reject \\
\hline topic759 & $3.0785 \mathrm{e}-05$ & $2.1423 \mathrm{e}-05$ & $2.22305 \mathrm{e}+06$ & $3.307 \mathrm{e}-18$ & Reject \\
\hline topic760 & 0.0001181 & 0.00012007 & $2.61445 \mathrm{e}+06$ & 0.37694 & Accept \\
\hline topic761 & $9.4506 \mathrm{e}-05$ & $5.2857 \mathrm{e}-05$ & $1.87041 \mathrm{e}+06$ & $4.801 \mathrm{e}-63$ & Reject \\
\hline topic762 & $3.5079 \mathrm{e}-05$ & $2.6288 \mathrm{e}-05$ & $2.19097 \mathrm{e}+06$ & $7.0135 \mathrm{e}-23$ & Reject \\
\hline topic763 & 0.00015539 & 0.00012781 & $2.09224 \mathrm{e}+06$ & $2.3038 \mathrm{e}-33$ & Reject \\
\hline topic764 & 0.00010969 & $7.9294 \mathrm{e}-05$ & $2.13276 \mathrm{e}+06$ & $1.7838 \mathrm{e}-28$ & Reject \\
\hline topic765 & $5.1866 \mathrm{e}-05$ & $1.8473 \mathrm{e}-05$ & $1.5804 \mathrm{e}+06$ & $8.3512 \mathrm{e}-119$ & Reject \\
\hline topic766 & 0.00010711 & $8.0862 \mathrm{e}-05$ & $2.22253 \mathrm{e}+06$ & $1.3357 \mathrm{e}-19$ & Reject \\
\hline topic767 & $4.9473 \mathrm{e}-05$ & $1.4138 \mathrm{e}-05$ & $1.47043 \mathrm{e}+06$ & $9.9035 \mathrm{e}-147$ & Reject \\
\hline topic768 & 0.00010985 & $5.6445 \mathrm{e}-05$ & $1.77481 \mathrm{e}+06$ & $5.9928 \mathrm{e}-80$ & Reject \\
\hline topic769 & $6.5113 \mathrm{e}-05$ & $4.3841 \mathrm{e}-05$ & $2.26588 \mathrm{e}+06$ & $3.1358 \mathrm{e}-16$ & Reject \\
\hline topic770 & $5.7068 \mathrm{e}-05$ & $2.3623 \mathrm{e}-05$ & $1.46001 \mathrm{e}+06$ & $6.1309 \mathrm{e}-150$ & Reject \\
\hline topic771 & $7.2901 \mathrm{e}-05$ & $6.2964 \mathrm{e}-05$ & $2.45772 \mathrm{e}+06$ & 0.00015381 & Reject \\
\hline topic772 & 0.00015018 & 0.00011304 & $1.87174 \mathrm{e}+06$ & $5.1011 \mathrm{e}-64$ & Reject \\
\hline topic773 & $3.7752 \mathrm{e}-05$ & $2.8132 \mathrm{e}-05$ & $2.13203 \mathrm{e}+06$ & $6.8971 \mathrm{e}-29$ & Reject \\
\hline topic774 & 0.00013634 & $7.8346 \mathrm{e}-05$ & $2.13944 \mathrm{e}+06$ & $5.4268 \mathrm{e}-28$ & Reject \\
\hline topic775 & 0.00020919 & 0.00018138 & $2.39634 \mathrm{e}+06$ & $1.3035 \mathrm{e}-07$ & Reject \\
\hline topic776 & $6.382 \mathrm{e}-05$ & $1.0588 \mathrm{e}-05$ & 736682 & 0 & Reject \\
\hline topic777 & $8.4125 \mathrm{e}-05$ & $6.222 \mathrm{e}-05$ & $2.23152 \mathrm{e}+06$ & $1.7481 \mathrm{e}-18$ & Reject \\
\hline topic778 & 0.00013479 & 0.00014692 & $2.46933 \mathrm{e}+06$ & 0.00019468 & Reject \\
\hline topic779 & 0.00011702 & $8.6542 \mathrm{e}-05$ & $2.16182 \mathrm{e}+06$ & $1.8847 \mathrm{e}-25$ & Reject \\
\hline topic780 & $2.7022 \mathrm{e}-05$ & $1.8075 \mathrm{e}-05$ & $2.23927 \mathrm{e}+06$ & $3.0211 \mathrm{e}-18$ & Reject \\
\hline topic781 & $8.5775 \mathrm{e}-05$ & $8.3876 \mathrm{e}-05$ & $2.61243 \mathrm{e}+06$ & 0.36994 & Accept \\
\hline
\end{tabular}


Table A1 - Continued from previous page

\begin{tabular}{|c|c|c|c|c|c|}
\hline Feature & EN median & FL median & u-value & p-value & $\mathbf{H}_{0}$ \\
\hline topic782 & 0.00011334 & 0.00010697 & $2.40876 \mathrm{e}+06$ & $4.26 \mathrm{e}-07$ & Reject \\
\hline topic783 & 0.00011604 & $8.0182 \mathrm{e}-05$ & $1.59887 \mathrm{e}+06$ & $4.0252 \mathrm{e}-117$ & Reject \\
\hline topic784 & $4.1026 \mathrm{e}-05$ & 0.00010459 & $1.68159 \mathrm{e}+06$ & $6.1915 \mathrm{e}-99$ & Reject \\
\hline topic785 & 0.00014975 & 0.0001726 & $2.39414 \mathrm{e}+06$ & $8.7629 \mathrm{e}-08$ & Reject \\
\hline topic786 & $8.5192 \mathrm{e}-05$ & $3.3504 \mathrm{e}-05$ & $1.31843 \mathrm{e}+06$ & $2.9784 \mathrm{e}-187$ & Reject \\
\hline topic787 & 0.00010106 & 0.00011344 & $2.49209 \mathrm{e}+06$ & 0.0012887 & Reject \\
\hline topic788 & 0.00012059 & $7.7762 \mathrm{e}-05$ & $2.065 \mathrm{e}+06$ & $2.2337 \mathrm{e}-36$ & Reject \\
\hline topic789 & $2.8263 \mathrm{e}-05$ & $3.3405 \mathrm{e}-05$ & $2.30835 \mathrm{e}+06$ & $4.7727 \mathrm{e}-13$ & Reject \\
\hline topic790 & 0.00012878 & 0.00011324 & $2.43826 \mathrm{e}+06$ & $1.117 \mathrm{e}-05$ & Reject \\
\hline topic791 & 0.00014117 & 0.00011773 & $2.37327 \mathrm{e}+06$ & 7.3763e-09 & Reject \\
\hline topic792 & $9.2667 \mathrm{e}-05$ & $9.2359 \mathrm{e}-05$ & $2.56044 \mathrm{e}+06$ & 0.071704 & Accept \\
\hline topic793 & 0.00010352 & $6.1295 \mathrm{e}-05$ & $1.74239 \mathrm{e}+06$ & $5.1993 \mathrm{e}-87$ & Reject \\
\hline topic794 & 0.00017466 & 0.00012727 & $2.14445 \mathrm{e}+06$ & $3.1162 \mathrm{e}-27$ & Reject \\
\hline topic795 & $8.6358 \mathrm{e}-05$ & $5.8565 \mathrm{e}-05$ & $1.7292 \mathrm{e}+06$ & $2.201 \mathrm{e}-89$ & Reject \\
\hline topic796 & $3.7104 \mathrm{e}-05$ & $2.0734 \mathrm{e}-05$ & $1.57335 \mathrm{e}+06$ & 7.0577e-123 & Reject \\
\hline topic797 & $5.5321 \mathrm{e}-05$ & $3.7977 \mathrm{e}-05$ & $1.98234 \mathrm{e}+06$ & $3.232 \mathrm{e}-47$ & Reject \\
\hline topic798 & 0.00013332 & 0.00012061 & $2.45456 \mathrm{e}+06$ & $6.5181 \mathrm{e}-05$ & Reject \\
\hline topic799 & 0.00014325 & $7.9582 \mathrm{e}-05$ & $1.32023 \mathrm{e}+06$ & $2.582 \mathrm{e}-186$ & Reject \\
\hline topic800 & $8.9747 \mathrm{e}-05$ & 0.00010825 & $2.39279 \mathrm{e}+06$ & $6.4999 \mathrm{e}-08$ & Reject \\
\hline topic801 & 0.00012782 & $9.4354 \mathrm{e}-05$ & $2.03372 \mathrm{e}+06$ & $1.4552 \mathrm{e}-40$ & Reject \\
\hline topic802 & $8.7691 \mathrm{e}-05$ & $5.8406 \mathrm{e}-05$ & $2.06755 \mathrm{e}+06$ & $4.5715 \mathrm{e}-36$ & Reject \\
\hline topic803 & 0.00013175 & $8.4087 \mathrm{e}-05$ & $1.88319 \mathrm{e}+06$ & $1.4216 \mathrm{e}-61$ & Reject \\
\hline topic804 & $9.0919 \mathrm{e}-05$ & $8.571 \mathrm{e}-05$ & $2.53774 \mathrm{e}+06$ & 0.022974 & Reject \\
\hline topic805 & $1.9518 \mathrm{e}-06$ & $1.7213 \mathrm{e}-06$ & $2.32094 \mathrm{e}+06$ & 4.1363e-12 & Reject \\
\hline topic806 & $3.8778 \mathrm{e}-05$ & $5.75 \mathrm{e}-05$ & $2.16928 \mathrm{e}+06$ & $3.3858 \mathrm{e}-23$ & Reject \\
\hline topic807 & 0.00013909 & $5.9611 \mathrm{e}-05$ & $1.64906 \mathrm{e}+06$ & $9.3695 \mathrm{e}-105$ & Reject \\
\hline topic808 & $3.014 \mathrm{e}-05$ & $5.055 \mathrm{e}-06$ & $1.59515 \mathrm{e}+06$ & $1.3718 \mathrm{e}-117$ & Reject \\
\hline topic809 & 0.00025779 & 0.0001946 & $1.92273 \mathrm{e}+06$ & $4.18 \mathrm{e}-56$ & Reject \\
\hline topic810 & $2.9309 \mathrm{e}-05$ & $2.1308 \mathrm{e}-05$ & $1.97359 \mathrm{e}+06$ & $1.8371 \mathrm{e}-48$ & Reject \\
\hline topic811 & 0.0001291 & $8.997 \mathrm{e}-05$ & $2.31877 \mathrm{e}+06$ & 7.691e-12 & Reject \\
\hline topic812 & $1.4848 \mathrm{e}-05$ & $8.7744 \mathrm{e}-06$ & $1.98499 \mathrm{e}+06$ & $7.5817 \mathrm{e}-47$ & Reject \\
\hline topic813 & $8.207 \mathrm{e}-05$ & $6.4385 \mathrm{e}-05$ & $2.31064 \mathrm{e}+06$ & $1.1503 \mathrm{e}-12$ & Reject \\
\hline topic814 & $6.6977 \mathrm{e}-05$ & $4.2883 \mathrm{e}-05$ & $1.79496 \mathrm{e}+06$ & $6.4613 \mathrm{e}-77$ & Reject \\
\hline topic815 & $8.5991 \mathrm{e}-05$ & $7.8935 \mathrm{e}-05$ & $2.49298 \mathrm{e}+06$ & 0.001366 & Reject \\
\hline topic816 & 0.00012538 & $5.2853 \mathrm{e}-05$ & $1.45101 \mathrm{e}+06$ & $3.1867 \mathrm{e}-151$ & Reject \\
\hline topic817 & $4.3771 \mathrm{e}-05$ & $3.7546 \mathrm{e}-06$ & 771152 & 0 & Reject \\
\hline topic818 & 0.00015245 & 0.00012635 & $2.23423 \mathrm{e}+06$ & $2.5017 \mathrm{e}-18$ & Reject \\
\hline topic819 & $8.6059 \mathrm{e}-05$ & $7.2078 \mathrm{e}-05$ & $2.25038 \mathrm{e}+06$ & $1.736 \mathrm{e}-17$ & Reject \\
\hline topic820 & $3.2549 \mathrm{e}-05$ & $2.4337 \mathrm{e}-05$ & $2.54514 \mathrm{e}+06$ & 0.35103 & Accept \\
\hline topic821 & 0.00012039 & $8.484 \mathrm{e}-05$ & $2.04723 \mathrm{e}+06$ & $1.0397 \mathrm{e}-38$ & Reject \\
\hline topic822 & $9.5323 \mathrm{e}-05$ & $4.572 \mathrm{e}-05$ & $1.49156 \mathrm{e}+06$ & 7.117e-142 & Reject \\
\hline topic823 & 0.00011984 & $7.459 \mathrm{e}-05$ & $2.09726 \mathrm{e}+06$ & $1.1573 \mathrm{e}-32$ & Reject \\
\hline
\end{tabular}


Table A1 - Continued from previous page

\begin{tabular}{|c|c|c|c|c|c|}
\hline Feature & EN median & FL median & u-value & p-value & $\mathrm{H}_{\mathbf{0}}$ \\
\hline topic824 & $1.8905 \mathrm{e}-05$ & $5.3878 \mathrm{e}-06$ & $1.85347 \mathrm{e}+06$ & $1.0237 \mathrm{e}-63$ & Reject \\
\hline topic825 & $3.4613 \mathrm{e}-05$ & $2.2603 \mathrm{e}-05$ & $1.87915 \mathrm{e}+06$ & $7.9875 \mathrm{e}-63$ & Reject \\
\hline topic826 & 0.00012764 & 0.00014358 & $2.40742 \mathrm{e}+06$ & $3.6569 \mathrm{e}-07$ & Reject \\
\hline topic827 & $9.3963 \mathrm{e}-05$ & $9.3347 \mathrm{e}-05$ & $2.62128 \mathrm{e}+06$ & 0.43679 & Accept \\
\hline topic828 & 0.00016805 & $7.9894 \mathrm{e}-05$ & $1.25045 \mathrm{e}+06$ & $5.4163 \mathrm{e}-207$ & Reject \\
\hline topic829 & 0.00022532 & 0.00023519 & $2.62293 \mathrm{e}+06$ & 0.46102 & Accept \\
\hline topic830 & $2.7144 \mathrm{e}-05$ & $3.1065 \mathrm{e}-05$ & $2.55866 \mathrm{e}+06$ & 0.072851 & Accept \\
\hline topic831 & 0.00013741 & 0.00011653 & $2.29661 \mathrm{e}+06$ & $9.7368 \mathrm{e}-14$ & Reject \\
\hline topic832 & 0.00011727 & 0.00011091 & $2.46246 \mathrm{e}+06$ & $9.7292 \mathrm{e}-05$ & Reject \\
\hline topic833 & 0.00013644 & 0.00010665 & $2.16541 \mathrm{e}+06$ & $2.3302 \mathrm{e}-24$ & Reject \\
\hline topic834 & $8.5989 \mathrm{e}-05$ & $4.1378 \mathrm{e}-05$ & $1.62378 \mathrm{e}+06$ & $1.4916 \mathrm{e}-110$ & Reject \\
\hline topic835 & 0.00014459 & 0.00011103 & $2.30198 \mathrm{e}+06$ & $9.3299 \mathrm{e}-13$ & Reject \\
\hline topic836 & 0.00012337 & $7.7163 \mathrm{e}-05$ & $2.00887 \mathrm{e}+06$ & $3.423 \mathrm{e}-43$ & Reject \\
\hline topi & $8.6315 \mathrm{e}-05$ & $7.926 \mathrm{e}-05$ & $2.52445 \mathrm{e}+06$ & 0.010948 & Reject \\
\hline & $4.3985 \mathrm{e}-05$ & $2.2144 \mathrm{e}-05$ & $1.84114 \mathrm{e}+06$ & $1.9653 \mathrm{e}-67$ & Reject \\
\hline topic839 & $6.2371 \mathrm{e}-05$ & $6.122 \mathrm{e}-05$ & $2.56765 \mathrm{e}+06$ & 0.087378 & Accept \\
\hline topic840 & $8.5215 \mathrm{e}-05$ & $8.9983 \mathrm{e}-05$ & $2.62218 \mathrm{e}+06$ & 0.45441 & Accept \\
\hline topic841 & 0.00012405 & 0.00013666 & $2.3948 \mathrm{e}+06$ & $8.2861 \mathrm{e}-08$ & Reject \\
\hline topic842 & $9.211 \mathrm{e}-05$ & $5.7793 \mathrm{e}-06$ & 856086 & 0 & Reject \\
\hline & 0.00010084 & & $2.33857 \mathrm{e}+06$ & $7.0944 \mathrm{e}-11$ & \\
\hline topic844 & $4.3772 \mathrm{e}-05$ & $3.2229 \mathrm{e}-06$ & $1.10723 \mathrm{e}+06$ & $2.0323 \mathrm{e}-251$ & Reject \\
\hline topic845 & 0.00011066 & $1.7387 \mathrm{e}-05$ & $1.36558 \mathrm{e}+06$ & $1.1546 \mathrm{e}-169$ & Reject \\
\hline topic846 & $9.4706 \mathrm{e}-05$ & $7.7666 \mathrm{e}-05$ & $2.40921 \mathrm{e}+06$ & $6.4935 \mathrm{e}-07$ & Reject \\
\hline top & $9.8539 \mathrm{e}-05$ & $8.1815 \mathrm{e}-05$ & $2.31749 \mathrm{e}+06$ & $2.0586 \mathrm{e}-12$ & Reject \\
\hline & & 8.37 & $2.54142 \mathrm{e}+06$ & 0.026063 & Reject \\
\hline topic849 & 0.00013481 & $8.2936 \mathrm{e}-05$ & $1.78577 \mathrm{e}+06$ & $6.5078 \mathrm{e}-79$ & Reject \\
\hline topic850 & 0.00016699 & 0.00013022 & $2.11706 \mathrm{e}+06$ & $2.643 \mathrm{e}-30$ & Reject \\
\hline topic851 & 0.00034899 & 0.00032694 & $2.44201 \mathrm{e}+06$ & $1.4464 \mathrm{e}-05$ & Reject \\
\hline topic852 & 0.00017401 & 0.00014471 & $2.10477 \mathrm{e}+06$ & $6.4377 \mathrm{e}-32$ & Reject \\
\hline & $5.3872 \mathrm{e}-05$ & $4.1372 \mathrm{e}-05$ & $2.32126 \mathrm{e}+06$ & 7.1193e-12 & Reject \\
\hline topi & $9.3926 \mathrm{e}-05$ & 0.00014416 & $2.01139 \mathrm{e}+06$ & $3.9363 \mathrm{e}-43$ & Reject \\
\hline topic855 & 0.00011203 & 0.00010537 & $2.50713 \mathrm{e}+06$ & 0.0031658 & Reject \\
\hline top & $8.9677 \mathrm{e}-05$ & 0.0001583 & $1.59389 \mathrm{e}+06$ & $4.8287 \mathrm{e}-118$ & Reject \\
\hline top & 0.00018066 & 0.00013464 & $2.10693 \mathrm{e}+06$ & $6.4819 \mathrm{e}-31$ & Reject \\
\hline topic858 & 0.0001019 & $8.3643 \mathrm{e}-05$ & $2.35505 \mathrm{e}+06$ & $4.673 \mathrm{e}-10$ & Reject \\
\hline & $5.5359 \mathrm{e}-05$ & $2.5931 \mathrm{e}-05$ & $1.25126 \mathrm{e}+06$ & $2.1963 \mathrm{e}-207$ & Reject \\
\hline topic860 & $7.4346 \mathrm{e}-05$ & $6.5924 \mathrm{e}-05$ & $2.36765 \mathrm{e}+06$ & $2.6216 \mathrm{e}-09$ & Reject \\
\hline topic861 & $7.3655 \mathrm{e}-05$ & $6.8062 \mathrm{e}-05$ & $2.58924 \mathrm{e}+06$ & 0.2286 & Accept \\
\hline topic862 & 0.00014604 & 0.00015237 & $2.60592 \mathrm{e}+06$ & 0.34463 & Accept \\
\hline topic863 & 0.00014926 & $7.8738 \mathrm{e}-05$ & $1.80276 \mathrm{e}+06$ & $2.2049 \mathrm{e}-75$ & Reject \\
\hline topic864 & 7.392e-05 & $3.9885 \mathrm{e}-05$ & $1.7051 \mathrm{e}+06$ & 2.6162e-94 & Reject \\
\hline topic865 & 0.00014404 & 0.00015843 & $2.57778 \mathrm{e}+06$ & 0.14535 & Accept \\
\hline
\end{tabular}


Table A1 - Continued from previous page

\begin{tabular}{|c|c|c|c|c|c|}
\hline Feature & EN median & FL median & u-value & p-value & $\mathrm{H}_{0}$ \\
\hline topic866 & $5.064 \mathrm{e}-05$ & $3.6991 \mathrm{e}-05$ & $2.10211 \mathrm{e}+06$ & $3.3065 \mathrm{e}-31$ & Reject \\
\hline topic867 & $8.6464 \mathrm{e}-05$ & $9.3815 \mathrm{e}-05$ & $2.53021 \mathrm{e}+06$ & 0.014268 & Reject \\
\hline topic868 & 0.00012593 & 0.0001771 & $2.00168 \mathrm{e}+06$ & $1.5042 \mathrm{e}-44$ & Reject \\
\hline topic869 & 0.00010908 & $6.5976 \mathrm{e}-05$ & $1.57395 \mathrm{e}+06$ & $2.3451 \mathrm{e}-122$ & Reject \\
\hline topic870 & $3.1748 \mathrm{e}-05$ & $1.3541 \mathrm{e}-05$ & $1.57567 \mathrm{e}+06$ & $2.0843 \mathrm{e}-121$ & Reject \\
\hline topic871 & $9.2494 \mathrm{e}-05$ & $3.1863 \mathrm{e}-05$ & $1.55294 \mathrm{e}+06$ & $3.1874 \mathrm{e}-126$ & Reject \\
\hline topic872 & 0.00014929 & 0.00010576 & $2.24887 \mathrm{e}+06$ & $2.8339 \mathrm{e}-17$ & Reject \\
\hline topic873 & 0.00010961 & $6.9184 \mathrm{e}-05$ & $1.56863 \mathrm{e}+06$ & $5.8647 \mathrm{e}-124$ & Reject \\
\hline topic874 & 0.00012758 & 0.00012816 & $2.59301 \mathrm{e}+06$ & 0.2073 & Accept \\
\hline topic875 & $9.5538 \mathrm{e}-05$ & 7.9902e-05 & $2.25177 \mathrm{e}+06$ & $1.843 \mathrm{e}-17$ & Reject \\
\hline topic876 & $3.4725 \mathrm{e}-05$ & $1.0657 \mathrm{e}-05$ & $1.85091 \mathrm{e}+06$ & $1.4576 \mathrm{e}-66$ & Reject \\
\hline topic877 & 0.00014499 & $8.7521 \mathrm{e}-05$ & $1.5268 \mathrm{e}+06$ & $9.6939 \mathrm{e}-134$ & Reject \\
\hline topic878 & 0.00010314 & 0.00013055 & $2.30463 \mathrm{e}+06$ & $2.1772 \mathrm{e}-13$ & Reject \\
\hline topic879 & 0.00011051 & $4.9135 \mathrm{e}-05$ & $1.80278 \mathrm{e}+06$ & $1.1188 \mathrm{e}-75$ & Reject \\
\hline topic880 & 0.00011771 & $3 e-05$ & $1.74778 \mathrm{e}+06$ & $8.2642 \mathrm{e}-86$ & Reject \\
\hline topic881 & $9.6696 \mathrm{e}-05$ & $4.9952 \mathrm{e}-05$ & $1.57726 \mathrm{e}+06$ & $1.9758 \mathrm{e}-121$ & Reject \\
\hline topic882 & $9.6561 \mathrm{e}-05$ & $7.2011 \mathrm{e}-05$ & $2.15638 \mathrm{e}+06$ & $2.5728 \mathrm{e}-26$ & Reject \\
\hline & $8.3012 \mathrm{e}-05$ & 5.18 & $2.14868 \mathrm{e}+06$ & $4.0811 \mathrm{e}-27$ & Reject \\
\hline topic884 & 0.00010147 & $9.9875 \mathrm{e}-05$ & $2.57411 \mathrm{e}+06$ & 0.11767 & Accept \\
\hline topic885 & 0.00013481 & 01041 & $2.11892 \mathrm{e}+06$ & $3.278 \mathrm{e}-30$ & Reject \\
\hline topic886 & $7.2113 \mathrm{e}-05$ & $5.5666 \mathrm{e}-05$ & $2.44345 \mathrm{e}+06$ & $2.0553 \mathrm{e}-05$ & Reject \\
\hline topic887 & 0.00010485 & $5.2061 \mathrm{e}-05$ & $1.73894 \mathrm{e}+06$ & $1.6198 \mathrm{e}-87$ & Reject \\
\hline & & 0.00011326 & $2.5301 \mathrm{e}+06$ & 0.017993 & Reject \\
\hline topic889 & 0.0001039 & $8.4424 \mathrm{e}-05$ & $2.22645 \mathrm{e}+06$ & $1.5767 \mathrm{e}-19$ & Reject \\
\hline topic890 & 0.00014568 & $9.3062 \mathrm{e}-05$ & $1.95766 \mathrm{e}+06$ & $5.0845 \mathrm{e}-51$ & Reject \\
\hline topic891 & 0.00010576 & $2.3821 \mathrm{e}-05$ & 861706 & 0 & Reject \\
\hline topic892 & $9.6344 \mathrm{e}-05$ & $9.021 \mathrm{e}-05$ & $2.53157 \mathrm{e}+06$ & 0.014429 & Reject \\
\hline & 0012212 & 05 & $2.09661 \mathrm{e}+06$ & $1.2478 \mathrm{e}-32$ & Reject \\
\hline topic894 & $8.4085 \mathrm{e}-05$ & $3.2214 \mathrm{e}-05$ & $1.36258 \mathrm{e}+06$ & $3.7023 \mathrm{e}-175$ & Reject \\
\hline topic895 & $5.278 \mathrm{e}-05$ & $1.6599 \mathrm{e}-06$ & 944410 & $2.0212 \mathrm{e}-304$ & Reject \\
\hline topic896 & 0.00012379 & 0.00010742 & $2.17024 \mathrm{e}+06$ & $6.5713 \mathrm{e}-25$ & Reject \\
\hline topi & $5.5779 \mathrm{e}-05$ & $1.0723 \mathrm{e}-05$ & 726075 & 0 & Reject \\
\hline topic898 & $4.5574 \mathrm{e}-05$ & $2.4961 \mathrm{e}-05$ & $1.68407 \mathrm{e}+06$ & $2.1576 \mathrm{e}-98$ & Reject \\
\hline 899 & 0.00014419 & 0.00011975 & $2.25482 \mathrm{e}+06$ & $8.6765 \mathrm{e}-17$ & Reject \\
\hline topic900 & 0.000115 & $7.503 \mathrm{e}-05$ & $1.99789 \mathrm{e}+06$ & $6.2572 \mathrm{e}-45$ & Reject \\
\hline topic901 & 0.00017695 & 0.0001297 & $2.09991 \mathrm{e}+06$ & $2.3382 \mathrm{e}-32$ & Reject \\
\hline topic902 & $1.6419 \mathrm{e}-08$ & $5.1788 \mathrm{e}-08$ & 751476 & $9.2238 \mathrm{e}-41$ & Reject \\
\hline topic903 & $7.9375 \mathrm{e}-05$ & 0.00011637 & $2.3572 \mathrm{e}+06$ & $7.2545 \mathrm{e}-10$ & Reject \\
\hline topic904 & $8.5474 \mathrm{e}-05$ & 0.0001206 & $2.17234 \mathrm{e}+06$ & $1.682 \mathrm{e}-24$ & Reject \\
\hline topic905 & $6.0106 \mathrm{e}-05$ & $4.8556 \mathrm{e}-05$ & $2.43864 \mathrm{e}+06$ & $1.1496 \mathrm{e}-05$ & Reject \\
\hline topic906 & $5.0711 \mathrm{e}-05$ & $4.4882 \mathrm{e}-05$ & $2.21506 \mathrm{e}+06$ & $1.2248 \mathrm{e}-20$ & Reject \\
\hline topic907 & 0.00014594 & 0.00010762 & $2.21018 \mathrm{e}+06$ & $8.4095 \mathrm{e}-21$ & Reject \\
\hline
\end{tabular}


Table A1 - Continued from previous page

\begin{tabular}{|c|c|c|c|c|c|}
\hline Feature & EN median & FL median & u-value & p-value & $\mathbf{H}_{0}$ \\
\hline topic908 & $9.7886 \mathrm{e}-05$ & $8.6827 \mathrm{e}-05$ & $2.33887 \mathrm{e}+06$ & $4.5612 \mathrm{e}-11$ & Reject \\
\hline topic909 & 0.00013234 & 0.00011493 & $2.35914 \mathrm{e}+06$ & $8.2486 \mathrm{e}-10$ & Reject \\
\hline topic910 & 0.00015628 & $8.3759 \mathrm{e}-05$ & $1.62236 \mathrm{e}+06$ & $1.1813 \mathrm{e}-110$ & Reject \\
\hline topic911 & 0.00018449 & 0.00014546 & $2.23135 \mathrm{e}+06$ & 7.9868e-19 & Reject \\
\hline topic912 & $6.3691 \mathrm{e}-05$ & $3.5561 \mathrm{e}-05$ & $1.64219 \mathrm{e}+06$ & $1.1413 \mathrm{e}-107$ & Reject \\
\hline topic913 & 0.00011918 & $8.3527 \mathrm{e}-05$ & $2.0581 \mathrm{e}+06$ & $2.3751 \mathrm{e}-37$ & Reject \\
\hline topic914 & 0.00015734 & 0.00021655 & $1.9986 \mathrm{e}+06$ & $3.0795 \mathrm{e}-45$ & Reject \\
\hline topic915 & 0.00015793 & 0.00012844 & $2.14842 \mathrm{e}+06$ & $6.319 \mathrm{e}-27$ & Reject \\
\hline topic916 & 0.00011042 & 0.00013722 & $2.43248 \mathrm{e}+06$ & $6.9348 \mathrm{e}-06$ & Reject \\
\hline topic917 & $9.2462 \mathrm{e}-05$ & $8.3303 \mathrm{e}-05$ & $2.46229 \mathrm{e}+06$ & 0.00010507 & Reject \\
\hline topic918 & 0.00024038 & 0.00019848 & $2.37285 \mathrm{e}+06$ & $6.0394 \mathrm{e}-09$ & Reject \\
\hline topic919 & $8.9418 \mathrm{e}-05$ & $5.6337 \mathrm{e}-05$ & $2.20208 \mathrm{e}+06$ & $1.2111 \mathrm{e}-21$ & Reject \\
\hline topic920 & $3.9814 \mathrm{e}-05$ & $1.9758 \mathrm{e}-05$ & $1.75127 \mathrm{e}+06$ & $2.5727 \mathrm{e}-85$ & Reject \\
\hline topic921 & 0.00013138 & 0.00010409 & $2.01619 \mathrm{e}+06$ & $7.2878 \mathrm{e}-43$ & Reject \\
\hline topic922 & $3.8766 \mathrm{e}-06$ & $1.897 \mathrm{e}-06$ & $1.82034 \mathrm{e}+06$ & $2.1267 \mathrm{e}-72$ & Reject \\
\hline topic923 & 0.00015279 & $9.7404 \mathrm{e}-05$ & $1.68427 \mathrm{e}+06$ & $1.0361 \mathrm{e}-98$ & Reject \\
\hline topic924 & $5.5978 \mathrm{e}-05$ & $5.793 \mathrm{e}-05$ & $2.51675 \mathrm{e}+06$ & 0.0073829 & Reject \\
\hline topic925 & $6.2823 \mathrm{e}-05$ & $1.8099 \mathrm{e}-05$ & $1.09020 \mathrm{e}+06$ & $2.7649 \mathrm{e}-255$ & Reject \\
\hline topic926 & $2.3346 \mathrm{e}-05$ & $8.7752 \mathrm{e}-06$ & $1.30649 \mathrm{e}+06$ & $7.6916 \mathrm{e}-191$ & Reject \\
\hline topic927 & 0.00010244 & $7.5071 \mathrm{e}-05$ & $2.16412 \mathrm{e}+06$ & $1.5896 \mathrm{e}-25$ & Reject \\
\hline topic928 & $6.428 \mathrm{e}-05$ & $3.9556 \mathrm{e}-05$ & $2.04022 \mathrm{e}+06$ & $1.6744 \mathrm{e}-38$ & Reject \\
\hline topic929 & 0.00014862 & 0.00018775 & $2.22859 \mathrm{e}+06$ & $1.9619 \mathrm{e}-19$ & Reject \\
\hline topic930 & $9.3202 \mathrm{e}-05$ & $9.368 \mathrm{e}-05$ & $2.62551 \mathrm{e}+06$ & 0.48486 & Accept \\
\hline topic931 & $7.9694 \mathrm{e}-05$ & 0.00013106 & $2.01978 \mathrm{e}+06$ & $2.4851 \mathrm{e}-38$ & Reject \\
\hline topic932 & 0.00013463 & 0.00011371 & $2.3611 \mathrm{e}+06$ & $1.2568 \mathrm{e}-09$ & Reject \\
\hline topic933 & 0.00012088 & $8.5728 \mathrm{e}-05$ & $2.26686 \mathrm{e}+06$ & $6.5219 \mathrm{e}-16$ & Reject \\
\hline topic934 & $2.3735 \mathrm{e}-05$ & $5.3043 \mathrm{e}-06$ & $1.45924 \mathrm{e}+06$ & $1.1845 \mathrm{e}-147$ & Reject \\
\hline topic935 & $7.6102 \mathrm{e}-05$ & $3.239 \mathrm{e}-05$ & $1.55554 \mathrm{e}+06$ & $1.9141 \mathrm{e}-125$ & Reject \\
\hline topic936 & $2.9554 \mathrm{e}-05$ & $5.8718 \mathrm{e}-06$ & $1.46238 \mathrm{e}+06$ & $6.2033 \mathrm{e}-149$ & Reject \\
\hline topic937 & $5.565 \mathrm{e}-05$ & $6.2413 \mathrm{e}-05$ & $2.49367 \mathrm{e}+06$ & 0.0013296 & Reject \\
\hline topic938 & 0.00010106 & $8.5665 \mathrm{e}-05$ & $2.36468 \mathrm{e}+06$ & 2.0163e-09 & Reject \\
\hline topic939 & 0.00012487 & 0.00010689 & $2.1264 \mathrm{e}+06$ & $2.1803 \mathrm{e}-29$ & Reject \\
\hline topic940 & $8.2818 \mathrm{e}-05$ & $6.8192 \mathrm{e}-05$ & $2.08394 \mathrm{e}+06$ & $3.132 \mathrm{e}-34$ & Reject \\
\hline topic941 & 0.00012963 & $7.3352 \mathrm{e}-05$ & $1.76958 \mathrm{e}+06$ & $6.7888 \mathrm{e}-82$ & Reject \\
\hline topic942 & $5.8171 \mathrm{e}-05$ & $3.3153 \mathrm{e}-05$ & $1.43432 \mathrm{e}+06$ & $9.6791 \mathrm{e}-156$ & Reject \\
\hline topic943 & $9.7063 \mathrm{e}-05$ & 0.00013505 & $2.06072 \mathrm{e}+06$ & $3.7623 \mathrm{e}-37$ & Reject \\
\hline topic944 & 0.00011725 & $9.7856 \mathrm{e}-05$ & $2.34344 \mathrm{e}+06$ & $1.2268 \mathrm{e}-10$ & Reject \\
\hline topic945 & 0.0001444 & 0.00014982 & $2.52101 \mathrm{e}+06$ & 0.0088667 & Reject \\
\hline topic946 & $8.9332 \mathrm{e}-05$ & $1.5428 \mathrm{e}-05$ & $1.75382 \mathrm{e}+06$ & $3.5503 \mathrm{e}-83$ & Reject \\
\hline topic947 & 0.00014985 & 0.00010655 & $2.17462 \mathrm{e}+06$ & $4.6462 \mathrm{e}-24$ & Reject \\
\hline topic948 & 0.00016217 & $7.845 \mathrm{e}-05$ & $1.3821 \mathrm{e}+06$ & $2.8384 \mathrm{e}-169$ & Reject \\
\hline topic949 & $7.0134 \mathrm{e}-05$ & $5.9628 \mathrm{e}-05$ & $2.38896 \mathrm{e}+06$ & $5.3448 \mathrm{e}-08$ & Reject \\
\hline
\end{tabular}


Table A1 - Continued from previous page

\begin{tabular}{|c|c|c|c|c|c|}
\hline Feature & EN median & FL median & u-value & p-value & $\mathbf{H}_{0}$ \\
\hline topic950 & 0.00014039 & 0.00016402 & $2.47713 \mathrm{e}+06$ & 0.0005732 & Reject \\
\hline topic951 & 0.00014591 & 0.00012051 & $2.36632 \mathrm{e}+06$ & $3.4236 \mathrm{e}-09$ & Reject \\
\hline topic952 & 0.00015459 & 0.00012135 & $2.19881 \mathrm{e}+06$ & $4.8756 \mathrm{e}-22$ & Reject \\
\hline topic953 & 0.00013247 & $8.7396 \mathrm{e}-05$ & $1.92241 \mathrm{e}+06$ & $7.3501 \mathrm{e}-56$ & Reject \\
\hline topic954 & 0.0001254 & $9.6437 \mathrm{e}-05$ & $1.93111 \mathrm{e}+06$ & $8.1075 \mathrm{e}-55$ & Reject \\
\hline topic955 & $6.068 \mathrm{e}-05$ & 0.00022 & $1.50344 \mathrm{e}+06$ & $7.4875 \mathrm{e}-138$ & Reject \\
\hline topic956 & $3.0931 \mathrm{e}-06$ & $3.5184 \mathrm{e}-06$ & $2.52606 \mathrm{e}+06$ & 0.173 & Accept \\
\hline topic957 & $9.2948 \mathrm{e}-05$ & $2.645 \mathrm{e}-05$ & $1.28869 \mathrm{e}+06$ & $3.553 \mathrm{e}-196$ & Reject \\
\hline topic958 & $6.5963 \mathrm{e}-05$ & $2.1834 \mathrm{e}-05$ & $1.3048 \mathrm{e}+06$ & $2.6547 \mathrm{e}-187$ & Reject \\
\hline topic959 & $5.9296 \mathrm{e}-05$ & $4.67 \mathrm{e}-06$ & $1.25692 \mathrm{e}+06$ & $1.7507 \mathrm{e}-205$ & Reject \\
\hline topic960 & $8.8096 \mathrm{e}-05$ & $6.5639 \mathrm{e}-05$ & $2.21573 \mathrm{e}+06$ & $2.1434 \mathrm{e}-20$ & Reject \\
\hline topic961 & $8.4845 \mathrm{e}-05$ & $4.8856 \mathrm{e}-05$ & $1.79386 \mathrm{e}+06$ & $1.9814 \mathrm{e}-77$ & Reject \\
\hline topic962 & 0.00017542 & 0.00013934 & $2.10429 \mathrm{e}+06$ & 7.4153e-32 & Reject \\
\hline topic963 & $8.7191 \mathrm{e}-05$ & $3.0703 \mathrm{e}-05$ & $1.69434 \mathrm{e}+06$ & $5.9448 \mathrm{e}-96$ & Reject \\
\hline topic964 & $9.6682 \mathrm{e}-05$ & 0.00010393 & $2.62756 \mathrm{e}+06$ & 0.49242 & Accept \\
\hline topic965 & 0.00016808 & 0.00012706 & $2.00223 \mathrm{e}+06$ & $1.2753 \mathrm{e}-44$ & Reject \\
\hline topic966 & 0.00014762 & 0.00015087 & $2.60875 \mathrm{e}+06$ & 0.35825 & Accept \\
\hline topic967 & 0.00012846 & $9.7926 \mathrm{e}-05$ & $1.94579 \mathrm{e}+06$ & $9.1565 \mathrm{e}-53$ & Reject \\
\hline topic968 & 0.00017425 & 0.00013465 & $1.90574 \mathrm{e}+06$ & $1.3781 \mathrm{e}-58$ & Reject \\
\hline topic969 & $5.8414 \mathrm{e}-05$ & $6.3192 \mathrm{e}-05$ & $2.5958 \mathrm{e}+06$ & 0.22555 & Accept \\
\hline topic970 & $1.6969 \mathrm{e}-05$ & 8.837e-06 & $1.87137 \mathrm{e}+06$ & $4.2956 \mathrm{e}-64$ & Reject \\
\hline topic971 & 0.00011589 & $8.7952 \mathrm{e}-05$ & $2.07307 \mathrm{e}+06$ & $1.2149 \mathrm{e}-35$ & Reject \\
\hline topic972 & $8.1979 \mathrm{e}-05$ & $3.0745 \mathrm{e}-05$ & $1.3818 \mathrm{e}+06$ & $9.4181 \mathrm{e}-170$ & Reject \\
\hline topic973 & $6.5944 \mathrm{e}-05$ & $5.7888 \mathrm{e}-05$ & $2.57062 \mathrm{e}+06$ & 0.11191 & Accept \\
\hline topic974 & 0.00012669 & 0.00011956 & $2.50254 \mathrm{e}+06$ & 0.0026921 & Reject \\
\hline topic975 & 0.00011503 & $7.2661 \mathrm{e}-05$ & $1.78195 \mathrm{e}+06$ & $2.8118 \mathrm{e}-79$ & Reject \\
\hline topic976 & 0.00014386 & 0.00015898 & $2.40838 \mathrm{e}+06$ & $5.2707 \mathrm{e}-07$ & Reject \\
\hline topic977 & 0.00019771 & 0.00021117 & $2.42764 \mathrm{e}+06$ & $3.7864 \mathrm{e}-06$ & Reject \\
\hline topic978 & 0.00015503 & 0.00013007 & $2.40834 \mathrm{e}+06$ & 7.4961e-07 & Reject \\
\hline topic979 & $5.1381 \mathrm{e}-05$ & $4.3577 \mathrm{e}-05$ & $2.35504 \mathrm{e}+06$ & $1.522 \mathrm{e}-09$ & Reject \\
\hline topic980 & $8.9447 \mathrm{e}-05$ & $7.2524 \mathrm{e}-05$ & $2.21987 \mathrm{e}+06$ & $3.3135 \mathrm{e}-20$ & Reject \\
\hline topic981 & 0.00010435 & $7.7576 \mathrm{e}-05$ & $2.1292 \mathrm{e}+06$ & $1.1756 \mathrm{e}-28$ & Reject \\
\hline topic982 & 0.00014845 & 0.00018383 & $2.31449 \mathrm{e}+06$ & $1.81 \mathrm{e}-12$ & Reject \\
\hline topic983 & $1.8321 \mathrm{e}-05$ & $1.2334 \mathrm{e}-05$ & $1.9328 \mathrm{e}+06$ & $1.05 \mathrm{e}-53$ & Reject \\
\hline topic984 & $3.6295 \mathrm{e}-05$ & $1.0967 \mathrm{e}-05$ & $1.19737 \mathrm{e}+06$ & $5.7532 \mathrm{e}-222$ & Reject \\
\hline topic985 & 0.00014739 & 0.0001184 & $2.14151 \mathrm{e}+06$ & $9.012 \mathrm{e}-28$ & Reject \\
\hline topic986 & 0.00010116 & 0.00010891 & $2.4378 \mathrm{e}+06$ & $1.0565 \mathrm{e}-05$ & Reject \\
\hline topic987 & $5.1522 \mathrm{e}-05$ & $3.768 \mathrm{e}-05$ & $2.35869 \mathrm{e}+06$ & $2.9305 \mathrm{e}-09$ & Reject \\
\hline topic988 & 8.8653e-05 & $5.7835 \mathrm{e}-05$ & $2.00197 \mathrm{e}+06$ & $2.1205 \mathrm{e}-44$ & Reject \\
\hline topic989 & $2.0456 \mathrm{e}-06$ & $2.4377 \mathrm{e}-06$ & $1.89862 \mathrm{e}+06$ & $5.8413 \mathrm{e}-05$ & Reject \\
\hline topic990 & 0.00012506 & $9.0681 \mathrm{e}-05$ & $1.81044 \mathrm{e}+06$ & $2.571 \mathrm{e}-74$ & Reject \\
\hline topic991 & $2.2736 \mathrm{e}-05$ & $6.6192 \mathrm{e}-05$ & $1.63686 \mathrm{e}+06$ & $1.1507 \mathrm{e}-102$ & Reject \\
\hline
\end{tabular}


Table A1 - Continued from previous page

\begin{tabular}{|c|c|c|c|c|c|}
\hline Feature & EN median & FL median & u-value & p-value & $\mathbf{H}_{0}$ \\
\hline topic992 & 0.00013231 & $8.6243 \mathrm{e}-05$ & $1.76465 \mathrm{e}+06$ & $8.4943 \mathrm{e}-83$ & Reject \\
\hline topic993 & $7.0397 \mathrm{e}-05$ & $6.7027 \mathrm{e}-05$ & $2.4641 \mathrm{e}+06$ & 0.00011244 & Reject \\
\hline topic994 & $3.8778 \mathrm{e}-05$ & $3.8028 \mathrm{e}-05$ & $2.40238 \mathrm{e}+06$ & $4.3412 \mathrm{e}-07$ & Reject \\
\hline topic995 & $9.371 \mathrm{e}-05$ & $6.6518 \mathrm{e}-05$ & $2.04181 \mathrm{e}+06$ & $3.8387 \mathrm{e}-39$ & Reject \\
\hline topic996 & $7.47 \mathrm{e}-05$ & $4.7662 \mathrm{e}-05$ & $1.68581 \mathrm{e}+06$ & $2.2165 \mathrm{e}-98$ & Reject \\
\hline topic997 & 0.00010873 & 0.00010403 & $2.54153 \mathrm{e}+06$ & 0.024796 & Reject \\
\hline topic998 & $9.6703 \mathrm{e}-05$ & $7.2613 \mathrm{e}-05$ & $2.42179 \mathrm{e}+06$ & $2.5943 \mathrm{e}-06$ & Reject \\
\hline topic999 & 0.00014031 & $9.7485 \mathrm{e}-05$ & $2.0458 \mathrm{e}+06$ & $6.8494 \mathrm{e}-39$ & Reject \\
\hline topic1000 & $8.9869 \mathrm{e}-05$ & $6.0533 \mathrm{e}-05$ & $1.7856 \mathrm{e}+06$ & 2.9061e-79 & Reject \\
\hline topic1001 & $7.2252 \mathrm{e}-05$ & $4.9425 \mathrm{e}-05$ & $2.20292 \mathrm{e}+06$ & $4.2656 \mathrm{e}-21$ & Reject \\
\hline topic1002 & $7.8228 \mathrm{e}-05$ & $5.0258 \mathrm{e}-05$ & $2.14113 \mathrm{e}+06$ & $8.4003 \mathrm{e}-28$ & Reject \\
\hline topic1003 & $6.2746 \mathrm{e}-05$ & $6.2007 \mathrm{e}-05$ & $2.60443 \mathrm{e}+06$ & 0.29642 & Accept \\
\hline topic1004 & 0.00012961 & 0.00012753 & $2.58928 \mathrm{e}+06$ & 0.18432 & Accept \\
\hline topic1005 & $2.536 \mathrm{e}-05$ & $9.1785 \mathrm{e}-05$ & $1.48234 \mathrm{e}+06$ & $3.9707 \mathrm{e}-131$ & Reject \\
\hline topic1006 & 0.00010829 & $6.5286 \mathrm{e}-05$ & $1.97161 \mathrm{e}+06$ & $5.2043 \mathrm{e}-49$ & Reject \\
\hline topic1007 & 0.00011571 & $8.4737 \mathrm{e}-05$ & $1.81597 \mathrm{e}+06$ & $8.1477 \mathrm{e}-74$ & Reject \\
\hline topic1008 & $7.9436 \mathrm{e}-05$ & $5.261 \mathrm{e}-05$ & $2.02689 \mathrm{e}+06$ & $4.4947 \mathrm{e}-41$ & Reject \\
\hline topic1009 & 0.00021377 & 0.00011987 & $1.69425 \mathrm{e}+06$ & $5.932 \mathrm{e}-96$ & Reject \\
\hline topic1010 & 0.00013883 & 0.00014944 & $2.49395 \mathrm{e}+06$ & 0.0017189 & Reject \\
\hline topic1011 & $7.4235 \mathrm{e}-05$ & $8.059 \mathrm{e}-05$ & $2.54424 \mathrm{e}+06$ & 0.037977 & Reject \\
\hline topic1012 & 0.00016132 & $7.3572 \mathrm{e}-05$ & $1.28113 \mathrm{e}+06$ & $9.1601 \mathrm{e}-198$ & Reject \\
\hline topic1013 & $1.0724 \mathrm{e}-05$ & $1.2195 \mathrm{e}-05$ & $2.43889 \mathrm{e}+06$ & 0.00043143 & Reject \\
\hline topic1014 & $1.7978 \mathrm{e}-05$ & $1.1076 \mathrm{e}-05$ & $2.00897 \mathrm{e}+06$ & $3.4371 \mathrm{e}-43$ & Reject \\
\hline topic1015 & 0.00012526 & 0.00010579 & $2.16578 \mathrm{e}+06$ & $3.7218 \mathrm{e}-25$ & Reject \\
\hline topic1016 & 0.00023608 & 0.00023792 & $2.61888 \mathrm{e}+06$ & 0.40545 & Accept \\
\hline topic1017 & 0.00013063 & $9.0865 \mathrm{e}-05$ & $2.08592 \mathrm{e}+06$ & $7.068 \mathrm{e}-34$ & Reject \\
\hline topic1018 & $4.8105 \mathrm{e}-05$ & 0.00011162 & $1.7005 \mathrm{e}+06$ & $3.2164 \mathrm{e}-93$ & Reject \\
\hline topic1019 & 0.00012555 & 0.00013151 & $2.5604 \mathrm{e}+06$ & 0.071271 & Accept \\
\hline topic1020 & $7.4382 \mathrm{e}-05$ & $6.606 \mathrm{e}-05$ & $2.25204 \mathrm{e}+06$ & $2.3816 \mathrm{e}-17$ & Reject \\
\hline topic1021 & $9.4378 \mathrm{e}-07$ & $7.4445 \mathrm{e}-07$ & $2.56956 \mathrm{e}+06$ & 0.31462 & Accept \\
\hline topic1022 & $4.4864 \mathrm{e}-05$ & $2.8742 \mathrm{e}-05$ & $2.02431 \mathrm{e}+06$ & $6.8336 \mathrm{e}-41$ & Reject \\
\hline topic1023 & $6.3067 \mathrm{e}-05$ & $5.6537 \mathrm{e}-05$ & $2.42095 \mathrm{e}+06$ & $4.1705 \mathrm{e}-06$ & Reject \\
\hline topic1024 & 0.00013302 & $8.9065 \mathrm{e}-05$ & $1.89888 \mathrm{e}+06$ & $1.1869 \mathrm{e}-59$ & Reject \\
\hline topic1025 & 0.00010329 & 0.00013948 & $2.42905 \mathrm{e}+06$ & $5.4813 \mathrm{e}-06$ & Reject \\
\hline topic1026 & 0.0001067 & $6.4256 \mathrm{e}-05$ & $1.71129 \mathrm{e}+06$ & $4.4632 \mathrm{e}-93$ & Reject \\
\hline topic1027 & 0.00018028 & $6.5163 \mathrm{e}-05$ & $1.15938 \mathrm{e}+06$ & $5.4493 \mathrm{e}-233$ & Reject \\
\hline topic1028 & 0.00014534 & 0.00010545 & $1.8562 \mathrm{e}+06$ & $9.2333 \mathrm{e}-67$ & Reject \\
\hline topic1029 & $7.2104 \mathrm{e}-05$ & $4.74 \mathrm{e}-05$ & $2.01697 \mathrm{e}+06$ & $1.253 \mathrm{e}-42$ & Reject \\
\hline topic1030 & $4.4953 \mathrm{e}-05$ & $3.4848 \mathrm{e}-05$ & $2.13212 \mathrm{e}+06$ & $1.9219 \mathrm{e}-28$ & Reject \\
\hline topic1031 & $6.0307 \mathrm{e}-05$ & $2.2914 \mathrm{e}-05$ & $1.53498 \mathrm{e}+06$ & $3.0093 \mathrm{e}-130$ & Reject \\
\hline topic1032 & 0.00012486 & 0.00010325 & $2.11244 \mathrm{e}+06$ & $6.1987 \mathrm{e}-31$ & Reject \\
\hline topic1033 & $2.6251 \mathrm{e}-05$ & $4.1184 \mathrm{e}-05$ & $2.03264 \mathrm{e}+06$ & $1.0528 \mathrm{e}-40$ & Reject \\
\hline
\end{tabular}


Table A1 - Continued from previous page

\begin{tabular}{|c|c|c|c|c|c|}
\hline Feature & EN median & FL median & u-value & p-value & $\mathbf{H}_{0}$ \\
\hline topic1034 & 0.00012452 & 0.00012067 & $2.55878 \mathrm{e}+06$ & 0.06024 & Accept \\
\hline topic1035 & 0.0001038 & $8.3953 \mathrm{e}-05$ & $2.23133 \mathrm{e}+06$ & $4.2134 \mathrm{e}-19$ & Reject \\
\hline topic1036 & $7.3886 \mathrm{e}-05$ & $6.1133 \mathrm{e}-05$ & $2.26648 \mathrm{e}+06$ & $3.4374 \mathrm{e}-16$ & Reject \\
\hline topic1037 & 0.00011996 & 0.00018701 & $1.88235 \mathrm{e}+06$ & $1.3423 \mathrm{e}-62$ & Reject \\
\hline topic1038 & 0.00011435 & $5.9308 \mathrm{e}-05$ & $2.06434 \mathrm{e}+06$ & $1.5667 \mathrm{e}-35$ & Reject \\
\hline topic1039 & $4.9834 \mathrm{e}-05$ & $3.5788 \mathrm{e}-05$ & $1.77405 \mathrm{e}+06$ & $3.1156 \mathrm{e}-81$ & Reject \\
\hline topic1040 & $1.9088 \mathrm{e}-05$ & $2.4637 \mathrm{e}-05$ & $2.34161 \mathrm{e}+06$ & $1.8342 \mathrm{e}-09$ & Reject \\
\hline topic1041 & 0.00015567 & 0.00017669 & $2.42386 \mathrm{e}+06$ & $2.2557 \mathrm{e}-06$ & Reject \\
\hline topic1042 & $4.2346 \mathrm{e}-05$ & $2.1786 \mathrm{e}-05$ & $1.67359 \mathrm{e}+06$ & $2.8052 \mathrm{e}-99$ & Reject \\
\hline topic1043 & $2.1784 \mathrm{e}-05$ & $2.6878 \mathrm{e}-06$ & $1.3767 \mathrm{e}+06$ & $6.9768 \mathrm{e}-169$ & Reject \\
\hline topic1044 & 0.00015139 & 0.00013418 & $2.2442 \mathrm{e}+06$ & $4.3067 \mathrm{e}-18$ & Reject \\
\hline topic1045 & 0.00010754 & $7.4124 \mathrm{e}-05$ & $1.87385 \mathrm{e}+06$ & $7.9169 \mathrm{e}-64$ & Reject \\
\hline topic1046 & $5.1692 \mathrm{e}-05$ & $4.0639 \mathrm{e}-05$ & $2.3678 \mathrm{e}+06$ & $3.5939 \mathrm{e}-09$ & Reject \\
\hline topic1047 & $4.8593 \mathrm{e}-05$ & $2.0703 \mathrm{e}-05$ & $1.65026 \mathrm{e}+06$ & $3.1746 \mathrm{e}-101$ & Reject \\
\hline topic1048 & 0.00011843 & 0.00010284 & $2.18855 \mathrm{e}+06$ & $5.1903 \mathrm{e}-23$ & Reject \\
\hline topic1049 & 0.00022741 & 0.0001743 & $2.03211 \mathrm{e}+06$ & $8.9988 \mathrm{e}-41$ & Reject \\
\hline topic1050 & 0.00013479 & 0.00012167 & $2.42857 \mathrm{e}+06$ & $3.7076 \mathrm{e}-06$ & Reject \\
\hline topic1051 & $7.0686 \mathrm{e}-05$ & $4.1345 \mathrm{e}-05$ & $1.55453 \mathrm{e}+06$ & $7.9769 \mathrm{e}-127$ & Reject \\
\hline topic1052 & $8.1145 \mathrm{e}-05$ & $6.7297 \mathrm{e}-05$ & $2.24078 \mathrm{e}+06$ & $3.2941 \mathrm{e}-18$ & Reject \\
\hline topic1053 & 0.00013821 & $8.6333 \mathrm{e}-05$ & $1.71952 \mathrm{e}+06$ & $1.8901 \mathrm{e}-91$ & Reject \\
\hline topic1054 & 0.0001176 & $7.8429 \mathrm{e}-05$ & $1.94981 \mathrm{e}+06$ & $1.2616 \mathrm{e}-51$ & Reject \\
\hline topic1055 & $9.6976 \mathrm{e}-05$ & $3.4297 \mathrm{e}-05$ & $2.13065 \mathrm{e}+06$ & $7.9256 \mathrm{e}-28$ & Reject \\
\hline topic1056 & $1.1031 \mathrm{e}-05$ & $1.101 \mathrm{e}-05$ & $2.61155 \mathrm{e}+06$ & 0.37259 & Accept \\
\hline topic1057 & $6.3858 \mathrm{e}-05$ & $4.7611 \mathrm{e}-05$ & $2.24065 \mathrm{e}+06$ & 7.0876e-18 & Reject \\
\hline topic1058 & $5.0562 \mathrm{e}-05$ & $3.526 \mathrm{e}-05$ & $2.25638 \mathrm{e}+06$ & $6.4834 \mathrm{e}-17$ & Reject \\
\hline topic1059 & $5.2899 \mathrm{e}-05$ & $6.437 \mathrm{e}-05$ & $2.38517 \mathrm{e}+06$ & $2.9168 \mathrm{e}-08$ & Reject \\
\hline topic1060 & $8.1391 \mathrm{e}-05$ & 0.00012724 & $2.18604 \mathrm{e}+06$ & $2.9122 \mathrm{e}-23$ & Reject \\
\hline topic1061 & 0.00011994 & $7.2093 \mathrm{e}-05$ & $1.81279 \mathrm{e}+06$ & $4.5951 \mathrm{e}-74$ & Reject \\
\hline topic1062 & $3.768 \mathrm{e}-05$ & $2.3797 \mathrm{e}-05$ & $1.71642 \mathrm{e}+06$ & $2.0561 \mathrm{e}-92$ & Reject \\
\hline topic1063 & 0.00013405 & $4.9982 \mathrm{e}-05$ & $1.13057 \mathrm{e}+06$ & $6.2087 \mathrm{e}-243$ & Reject \\
\hline topic1064 & 0.00014177 & 0.00011478 & $2.20165 \mathrm{e}+06$ & $2.6089 \mathrm{e}-21$ & Reject \\
\hline topic1065 & $7.9857 \mathrm{e}-08$ & $8.199 \mathrm{e}-08$ & 273624 & 0.2761 & Accept \\
\hline topic1066 & 0.00010657 & 0.00010437 & $2.58371 \mathrm{e}+06$ & 0.15945 & Accept \\
\hline topic1067 & 0.00022354 & 0.00016232 & $2.00813 \mathrm{e}+06$ & $8.033 \mathrm{e}-44$ & Reject \\
\hline topic1068 & 0.00013554 & $8.8757 \mathrm{e}-05$ & $1.95976 \mathrm{e}+06$ & $1.9657 \mathrm{e}-50$ & Reject \\
\hline topic1069 & $6.0736 \mathrm{e}-05$ & $6.7845 \mathrm{e}-06$ & 959818 & 7.3593e-301 & Reject \\
\hline topic1070 & $8.9241 \mathrm{e}-05$ & 0.00011286 & $2.12513 \mathrm{e}+06$ & $1.2205 \mathrm{e}-29$ & Reject \\
\hline topic1071 & 0.00014653 & 0.00013041 & $2.4249 \mathrm{e}+06$ & $8.589 \mathrm{e}-06$ & Reject \\
\hline topic1072 & $6.9675 \mathrm{e}-05$ & $6.8858 \mathrm{e}-05$ & $2.57784 \mathrm{e}+06$ & 0.14719 & Accept \\
\hline topic1073 & $1.0817 \mathrm{e}-05$ & $3.8515 \mathrm{e}-05$ & $1.63018 \mathrm{e}+06$ & $5.1731 \mathrm{e}-106$ & Reject \\
\hline topic1074 & 0.00011005 & $8.1183 \mathrm{e}-05$ & $2.27239 \mathrm{e}+06$ & $1.4541 \mathrm{e}-15$ & Reject \\
\hline topic1075 & 0.00010699 & 0.00010568 & $2.57361 \mathrm{e}+06$ & 0.12653 & Accept \\
\hline
\end{tabular}

Continued on next page 
Table A1 - Continued from previous page

\begin{tabular}{|c|c|c|c|c|c|}
\hline Feature & EN median & FL median & u-value & p-value & $\mathbf{H}_{0}$ \\
\hline topic1076 & $8.9391 \mathrm{e}-05$ & $7.0407 \mathrm{e}-05$ & $2.12999 \mathrm{e}+06$ & $4.1461 \mathrm{e}-29$ & Reject \\
\hline topic1077 & 0.00018393 & 0.00018925 & $2.52526 \mathrm{e}+06$ & 0.011412 & Reject \\
\hline topic1078 & $9.6036 \mathrm{e}-05$ & $7.0841 \mathrm{e}-05$ & $2.06498 \mathrm{e}+06$ & $8.6923 \mathrm{e}-36$ & Reject \\
\hline topic1079 & $5.9931 \mathrm{e}-05$ & $4.498 \mathrm{e}-05$ & $2.17954 \mathrm{e}+06$ & $6.9438 \mathrm{e}-24$ & Reject \\
\hline topic1080 & $4.354 \mathrm{e}-05$ & $3.6826 \mathrm{e}-05$ & $2.32521 \mathrm{e}+06$ & $1.1182 \mathrm{e}-11$ & Reject \\
\hline topic1081 & $2.187 \mathrm{e}-05$ & $1.6266 \mathrm{e}-05$ & $2.15544 \mathrm{e}+06$ & $3.3691 \mathrm{e}-26$ & Reject \\
\hline topic1082 & $4.844 \mathrm{e}-05$ & $3.1305 \mathrm{e}-05$ & $2.16828 \mathrm{e}+06$ & $6.7477 \mathrm{e}-24$ & Reject \\
\hline topic1083 & $1.8229 \mathrm{e}-05$ & $1.562 \mathrm{e}-05$ & $2.41399 \mathrm{e}+06$ & $2.8947 \mathrm{e}-06$ & Reject \\
\hline topic1084 & $6.0236 \mathrm{e}-05$ & $5.5578 \mathrm{e}-05$ & $2.48034 \mathrm{e}+06$ & 0.00048031 & Reject \\
\hline topic1085 & 0.00010126 & $5.1549 \mathrm{e}-05$ & $1.49252 \mathrm{e}+06$ & $1.172 \mathrm{e}-141$ & Reject \\
\hline topic1086 & 0.00010178 & $7.5503 \mathrm{e}-05$ & $2.23173 \mathrm{e}+06$ & $5.5363 \mathrm{e}-19$ & Reject \\
\hline topic1087 & 0.0001213 & 0.00012699 & $2.48464 \mathrm{e}+06$ & 0.00061554 & Reject \\
\hline topic1088 & $9.9408 \mathrm{e}-05$ & $5.1546 \mathrm{e}-05$ & $1.86674 \mathrm{e}+06$ & $7.6833 \mathrm{e}-65$ & Reject \\
\hline topic1089 & 0.00013185 & 0.00013264 & $2.61915 \mathrm{e}+06$ & 0.45933 & Accept \\
\hline topic1090 & $9.4214 \mathrm{e}-05$ & $6.4128 \mathrm{e}-05$ & $1.72953 \mathrm{e}+06$ & $3.6759 \mathrm{e}-89$ & Reject \\
\hline topic1091 & $8.2797 \mathrm{e}-05$ & $6.7448 \mathrm{e}-05$ & $2.31137 \mathrm{e}+06$ & $7.6539 \mathrm{e}-13$ & Reject \\
\hline topic1092 & 0.00012877 & $6.0955 \mathrm{e}-05$ & $1.51655 \mathrm{e}+06$ & $4.7984 \mathrm{e}-135$ & Reject \\
\hline topic1093 & $5.1474 \mathrm{e}-05$ & $2.2235 \mathrm{e}-05$ & $1.83232 \mathrm{e}+06$ & $7.1302 \mathrm{e}-70$ & Reject \\
\hline topic1094 & 0.00014889 & 0.00014857 & $2.52601 \mathrm{e}+06$ & 0.011202 & Reject \\
\hline topic1095 & $1.8481 \mathrm{e}-06$ & $1.8411 \mathrm{e}-06$ & $2.56635 \mathrm{e}+06$ & 0.10398 & Accept \\
\hline topic1096 & $5.2749 \mathrm{e}-05$ & $3.8955 \mathrm{e}-05$ & $2.27243 \mathrm{e}+06$ & $1.228 \mathrm{e}-15$ & Reject \\
\hline topic1097 & 0.00010878 & $7.8405 \mathrm{e}-05$ & $2.14126 \mathrm{e}+06$ & $1.8043 \mathrm{e}-27$ & Reject \\
\hline topic1098 & 0.00016074 & 0.00012167 & $2.22591 \mathrm{e}+06$ & $1.7197 \mathrm{e}-19$ & Reject \\
\hline topic1099 & 0.00020419 & 0.00023486 & $2.35691 \mathrm{e}+06$ & $6.055 \mathrm{e}-10$ & Reject \\
\hline topic1100 & 0.0002106 & 0.00019449 & $2.47683 \mathrm{e}+06$ & 0.0003302 & Reject \\
\hline topic1101 & $6.8308 \mathrm{e}-05$ & $7.2827 \mathrm{e}-05$ & $2.49596 \mathrm{e}+06$ & 0.0017071 & Reject \\
\hline topic1102 & 0.00010245 & $5.8598 \mathrm{e}-05$ & $1.64663 \mathrm{e}+06$ & $2.3517 \mathrm{e}-106$ & Reject \\
\hline topic1103 & 0.0001466 & 0.00015365 & $2.57384 \mathrm{e}+06$ & 0.1165 & Accept \\
\hline topic1104 & 0.00012551 & 7.7066e-05 & $1.78614 \mathrm{e}+06$ & $7.8802 \mathrm{e}-79$ & Reject \\
\hline topic1105 & 0.00013911 & 0.00010807 & $2.34975 \mathrm{e}+06$ & $4.8231 \mathrm{e}-10$ & Reject \\
\hline topic1106 & 0.00011119 & $4.0773 \mathrm{e}-05$ & $1.297 \mathrm{e}+06$ & $8.8764 \mathrm{e}-194$ & Reject \\
\hline topic1107 & 0.00011547 & 0.00010508 & $2.37938 \mathrm{e}+06$ & $1.403 \mathrm{e}-08$ & Reject \\
\hline topic1108 & $2.727 \mathrm{e}-05$ & $3.4693 \mathrm{e}-05$ & $2.48234 \mathrm{e}+06$ & 0.0006628 & Reject \\
\hline topic1109 & 0.00010615 & $7.2915 \mathrm{e}-05$ & $2.31927 \mathrm{e}+06$ & $3.2325 \mathrm{e}-12$ & Reject \\
\hline topic1110 & 0.00013009 & $6.626 \mathrm{e}-05$ & $1.68869 \mathrm{e}+06$ & $2.9466 \mathrm{e}-97$ & Reject \\
\hline topic1111 & 0.00013196 & $9.5635 \mathrm{e}-05$ & $2.09015 \mathrm{e}+06$ & $2.2136 \mathrm{e}-33$ & Reject \\
\hline topic1112 & $4.3569 \mathrm{e}-05$ & $1.1337 \mathrm{e}-05$ & $1.33324 \mathrm{e}+06$ & $5.9229 \mathrm{e}-180$ & Reject \\
\hline topic1113 & 0.00010992 & $7.6758 \mathrm{e}-05$ & $2.40032 \mathrm{e}+06$ & $1.6016 \mathrm{e}-07$ & Reject \\
\hline topic1114 & 0.00015456 & 0.00013978 & $2.41817 \mathrm{e}+06$ & $1.7068 \mathrm{e}-06$ & Reject \\
\hline topic1115 & 0.00012224 & $8.0727 \mathrm{e}-05$ & $2.05031 \mathrm{e}+06$ & $5.7406 \mathrm{e}-38$ & Reject \\
\hline topic1116 & $9.295 \mathrm{e}-05$ & $5.0123 \mathrm{e}-05$ & $1.86677 \mathrm{e}+06$ & $5.4469 \mathrm{e}-65$ & Reject \\
\hline topic1117 & 0.00013472 & 0.00010248 & $1.96372 \mathrm{e}+06$ & $5.3033 \mathrm{e}-50$ & Reject \\
\hline
\end{tabular}


Table A1 - Continued from previous page

\begin{tabular}{|c|c|c|c|c|c|}
\hline Feature & EN median & FL median & u-value & p-value & $\mathrm{H}_{0}$ \\
\hline topic1118 & 0.00010165 & $4.214 \mathrm{e}-05$ & $1.45436 \mathrm{e}+06$ & $2.2763 \mathrm{e}-151$ & Reject \\
\hline topic1119 & 0.00018955 & $9.5194 \mathrm{e}-05$ & $1.87084 \mathrm{e}+06$ & $4.8633 \mathrm{e}-64$ & Reject \\
\hline topic1120 & 0.00010521 & 7.3331e-05 & $2.03249 \mathrm{e}+06$ & $1.008 \mathrm{e}-40$ & Reject \\
\hline topic1121 & 8849 & 0.00019513 & $2.52979 \mathrm{e}+06$ & 938 & Reject \\
\hline topic1122 & 0.00012397 & $7.72 \mathrm{e}-05$ & $1.60438 \mathrm{e}+06$ & $1.6197 \mathrm{e}-115$ & Reject \\
\hline topic1123 & $8.126 \mathrm{e}-05$ & $4.8496 \mathrm{e}-05$ & $1.70887 \mathrm{e}+06$ & $9.489 \mathrm{e}-94$ & Reject \\
\hline topic1124 & $6.1639 \mathrm{e}-05$ & $8.6174 \mathrm{e}-05$ & $2.12503 \mathrm{e}+06$ & $2.0019 \mathrm{e}-29$ & Reject \\
\hline topic1125 & $3.9096 \mathrm{e}-05$ & $2.4592 \mathrm{e}-05$ & $1.82378 \mathrm{e}+06$ & $2.7682 \mathrm{e}-72$ & Reject \\
\hline topic1126 & 0.00012638 & $7.9559 \mathrm{e}-05$ & $1.8145 \mathrm{e}+06$ & $1.3834 \mathrm{e}-73$ & Reject \\
\hline topic1127 & 0.00013093 & 0.00012586 & $2.62426 \mathrm{e}+06$ & 0.47288 & Accept \\
\hline topic1128 & 0.00013535 & 0.00014234 & $2.50587 \mathrm{e}+06$ & 0.0031227 & Reject \\
\hline topic1129 & 0.00012264 & 0.00010397 & $2.41605 \mathrm{e}+06$ & $1.2246 \mathrm{e}-06$ & Reject \\
\hline topic1130 & 0.00013327 & $9.3538 \mathrm{e}-05$ & $2.39113 \mathrm{e}+06$ & $6.8865 \mathrm{e}-08$ & Reject \\
\hline topic1131 & $4.4951 \mathrm{e}-05$ & $2.3567 \mathrm{e}-05$ & $1.6509 \mathrm{e}+06$ & $8.1129 \mathrm{e}-106$ & Reject \\
\hline topic1132 & 0.0001124 & $508 \mathrm{e}-05$ & $2.22562 \mathrm{e}+06$ & $3.8248 \mathrm{e}-19$ & ieject \\
\hline topic1133 & 0.00013976 & 0.00013628 & $2.51336 \mathrm{e}+06$ & 0.0047821 & Reject \\
\hline topic1134 & 0.00018093 & 0.00017208 & $2.48764 \mathrm{e}+06$ & 0.00092496 & Reject \\
\hline & 0.00011308 & $42 \mathrm{e}-05$ & $1.22614 \mathrm{e}+06$ & $1.0383 \mathrm{e}-214$ & Reject \\
\hline topic1136 & 0.00012414 & 0.00010322 & $2.22861 \mathrm{e}+06$ & $4.4535 \mathrm{e}-19$ & Reject \\
\hline topic1137 & $.733 \mathrm{e}-05$ & $716 \mathrm{e}-05$ & $2.13674 \mathrm{e}+06$ & $4.7609 \mathrm{e}-28$ & Reject \\
\hline topic1138 & $6.0055 \mathrm{e}-05$ & $5.7543 \mathrm{e}-05$ & $2.55633 \mathrm{e}+06$ & 0.059987 & Accept \\
\hline topic1139 & 0.00012733 & 0.00015603 & $2.29021 \mathrm{e}+06$ & $.3113 \mathrm{e}-14$ & Reject \\
\hline & $78 \mathrm{e}-05$ & $2009 \mathrm{e}$ & $2.23057 \mathrm{e}+06$ & & \\
\hline topic1141 & $9.2788 \mathrm{e}-05$ & $8.2613 \mathrm{e}-05$ & $2.32214 \mathrm{e}+06$ & $3.5969 \mathrm{e}-12$ & Reject \\
\hline topic1142 & 0.00012796 & $8.9281 \mathrm{e}-05$ & $1.96127 \mathrm{e}+06$ & $2.3486 \mathrm{e}-50$ & Reject \\
\hline topic1143 & $5 e-05$ & $5.604 \mathrm{e}-05$ & $2.4196 \mathrm{e}+06$ & $2.5583 \mathrm{e}-06$ & Reject \\
\hline topic1144 & $4.0814 \mathrm{e}-05$ & $5.3715 \mathrm{e}-06$ & 927296 & 0 & Reject \\
\hline & & 0.0002964 & $2.14715 \mathrm{e}+06$ & $2.8209 \mathrm{e}-27$ & Reject \\
\hline topic1146 & $5.3844 \mathrm{e}-05$ & $3.6765 \mathrm{e}-05$ & $2.10021 \mathrm{e}+06$ & $2.53 \mathrm{e}-32$ & Reject \\
\hline topic1147 & 0.00013368 & 0.00010905 & $2.40554 \mathrm{e}+06$ & $3.7716 \mathrm{e}-07$ & Reject \\
\hline 148 & $8.9447 \mathrm{e}-05$ & $6.5657 \mathrm{e}-05$ & $2.16985 \mathrm{e}+06$ & $1.2088 \mathrm{e}-24$ & Reject \\
\hline topic1149 & $5.8268 \mathrm{e}-05$ & $3.7656 \mathrm{e}-05$ & $2.22207 \mathrm{e}+06$ & $7.7124 \mathrm{e}-19$ & Reject \\
\hline 1150 & $2.6605 \mathrm{e}-05$ & 1.947 & $2.2035 \mathrm{e}+06$ & $4.2104 \mathrm{e}-20$ & Reject \\
\hline 1151 & $5.8984 \mathrm{e}-05$ & $7.0141 \mathrm{e}-05$ & $2.49007 \mathrm{e}+06$ & 0.0010937 & Reject \\
\hline topic1152 & $8.2236 \mathrm{e}-05$ & $6.24 \mathrm{e}-05$ & $2.25213 \mathrm{e}+06$ & $2.9145 \mathrm{e}-17$ & Reject \\
\hline 153 & $9.5502 \mathrm{e}-05$ & 7.0931e-05 & $2.23418 \mathrm{e}+06$ & $6.0154 \mathrm{e}-19$ & Reject \\
\hline topic1154 & $6.3162 \mathrm{e}-05$ & $3.6707 \mathrm{e}-05$ & $1.64298 \mathrm{e}+06$ & $2.4709 \mathrm{e}-107$ & Reject \\
\hline topic1155 & 0.00014087 & $6.5491 \mathrm{e}-05$ & $1.53132 \mathrm{e}+06$ & $1.8049 \mathrm{e}-132$ & Reject \\
\hline topic1156 & 0.00017473 & 0.00013144 & $1.98795 \mathrm{e}+06$ & $3.5668 \mathrm{e}-46$ & Reject \\
\hline topic1157 & 0.00013833 & 0.00010397 & $2.37221 \mathrm{e}+06$ & $4.7983 \mathrm{e}-09$ & Reject \\
\hline topic1158 & 0.00013415 & $8.15 \mathrm{e}-05$ & $1.73534 \mathrm{e}+06$ & $7.022 \mathrm{e}-88$ & Reject \\
\hline topic1159 & 0.00013377 & 0.00010997 & $2.19411 \mathrm{e}+06$ & $2.2109 \mathrm{e}-22$ & Reject \\
\hline
\end{tabular}


Table A1 - Continued from previous page

\begin{tabular}{|c|c|c|c|c|c|}
\hline Feature & EN median & FL median & u-value & p-value & $\mathrm{H}_{0}$ \\
\hline topic1160 & $1.6235 \mathrm{e}-05$ & $2.8302 \mathrm{e}-05$ & $2.13004 \mathrm{e}+06$ & $3.0447 \mathrm{e}-28$ & Reject \\
\hline topic1161 & 0.00011773 & 0.00011063 & $2.55893 \mathrm{e}+06$ & 0.063591 & Accept \\
\hline topic1162 & 0.00015318 & $9.9337 \mathrm{e}-05$ & $2.39773 \mathrm{e}+06$ & $4.7411 \mathrm{e}-07$ & Reject \\
\hline topic1163 & $3 e-05$ & $3.3705 \mathrm{e}-05$ & $2.05675 \mathrm{e}+06$ & $2.1529 \mathrm{e}-37$ & eject \\
\hline topic1164 & $3.1302 \mathrm{e}-05$ & $1.93 \mathrm{e}-05$ & $2.1077 \mathrm{e}+06$ & $3.102 \mathrm{e}-30$ & Reject \\
\hline topic1165 & 0.00017197 & 0.00013427 & $2.18185 \mathrm{e}+06$ & $2.2637 \mathrm{e}-23$ & Reject \\
\hline topic1166 & 0.00013367 & $7.2732 \mathrm{e}-05$ & $1.76448 \mathrm{e}+06$ & $2.3641 \mathrm{e}-82$ & Reject \\
\hline topic1167 & 0.00011425 & $8.601 \mathrm{e}-05$ & $2.11338 \mathrm{e}+06$ & $1.0036 \mathrm{e}-30$ & Reject \\
\hline topic1168 & 0.00014332 & 0.00010628 & $1.95208 \mathrm{e}+06$ & $1.4922 \mathrm{e}-51$ & Reject \\
\hline topic1169 & $7.7149 \mathrm{e}-05$ & $5.4838 \mathrm{e}-05$ & $1.73812 \mathrm{e}+06$ & 50853 & Reject \\
\hline topic1170 & 0.00010282 & $7.1313 \mathrm{e}-05$ & $1.73477 \mathrm{e}+06$ & $1.1915 \mathrm{e}-88$ & Reject \\
\hline topic1171 & $8862 \mathrm{e}-05$ & $4.8517 \mathrm{e}-05$ & $1.76013 \mathrm{e}+06$ & $2.5559 \mathrm{e}-83$ & Reject \\
\hline topic1172 & $6.1947 \mathrm{e}-05$ & $6.2482 \mathrm{e}-05$ & $2.62829 \mathrm{e}+06$ & 0.49892 & Accept \\
\hline topic1173 & $5.2012 \mathrm{e}-05$ & $4.0139 \mathrm{e}-05$ & $2.24246 \mathrm{e}+06$ & $8.4777 \mathrm{e}-18$ & Reject \\
\hline topic1174 & 0.00011383 & $5 e-05$ & $2.23306 \mathrm{e}+06$ & $348 \mathrm{e}-19$ & eject \\
\hline topic1175 & 0.00010267 & $9.5054 \mathrm{e}-05$ & $2.40242 \mathrm{e}+06$ & $2.0491 \mathrm{e}-07$ & Reject \\
\hline topic1176 & $1041 \mathrm{e}-05$ & $8.8816 \mathrm{e}-06$ & $1.63549 \mathrm{e}+06$ & $3.265 \mathrm{e}-108$ & Reject \\
\hline & 0.00013212 & 05 & $1.78006 \mathrm{e}+06$ & $2.8088 \mathrm{e}-80$ & \\
\hline topic1178 & $5.6461 \mathrm{e}-06$ & $9.0363 \mathrm{e}-06$ & $1.83815 \mathrm{e}+06$ & $1.7852 \mathrm{e}-18$ & Reject \\
\hline topic1179 & 0.00 & $7 \mathrm{e}-05$ & $494 \mathrm{e}+06$ & 29 & Reject \\
\hline topic1180 & $9383 \mathrm{e}-05$ & $3.4347 \mathrm{e}-05$ & $2.26804 \mathrm{e}+06$ & $6.7542 \mathrm{e}-16$ & Reject \\
\hline topic1181 & $3.9027 \mathrm{e}-05$ & $8 \mathrm{e}-05$ & $1.81038 \mathrm{e}+06$ & $1.783 \mathrm{e}-74$ & Reject \\
\hline & & 0.00011861 & $2.35584 \mathrm{e}+06$ & 5.2204 & \\
\hline topic1183 & $6.8139 \mathrm{e}-05$ & $3 \mathrm{e}-05$ & $2.18556 \mathrm{e}+06$ & $1 e-23$ & Reject \\
\hline topic1184 & 0.00013044 & $8.4063 \mathrm{e}-05$ & $1.79095 \mathrm{e}+06$ & $8.3381 \mathrm{e}-78$ & Reject \\
\hline topic1185 & 0.00013371 & $5 \mathrm{e}-05$ & $1.86673 \mathrm{e}+06$ & $1.0597 \mathrm{e}-64$ & Reject \\
\hline topic1186 & 1567 & $4 \mathrm{e}-05$ & $2.12483 \mathrm{e}+06$ & $1.4661 \mathrm{e}-29$ & Reject \\
\hline & & & $1.82979 \mathrm{e}+06$ & -67 & \\
\hline topic1188 & $e-05$ & $9.2144 \mathrm{e}-05$ & $2.5532 \mathrm{e}+06$ & 0.046761 & Reject \\
\hline topic1189 & 0.00013535 & 0.00012579 & $2.4906 \mathrm{e}+06$ & 0.0014669 & Reject \\
\hline 1190 & $438 \mathrm{e}-05$ & $7 \mathrm{e}-05$ & 646060 & 0 & Reject \\
\hline topic1191 & 0.0001141 & 0.00011579 & $2.61709 \mathrm{e}+06$ & 0.39008 & Accept \\
\hline 192 & $5.8537 \mathrm{e}-05$ & e- 05 & $1.72134 \mathrm{e}+06$ & $1.92 \mathrm{e}-91$ & Reject \\
\hline 1193 & $7.3478 \mathrm{e}-05$ & $3.7295 \mathrm{e}-05$ & $1.64592 \mathrm{e}+06$ & $1.5333 \mathrm{e}-106$ & Reject \\
\hline topic1194 & $4.1063 \mathrm{e}-05$ & 0.0001442 & $1.45018 \mathrm{e}+06$ & $1.7936 \mathrm{e}-151$ & Reject \\
\hline & $7.7884 \mathrm{e}-05$ & $9 \mathrm{e}-05$ & $2.36813 \mathrm{e}+06$ & $3.2411 \mathrm{e}-09$ & Reject \\
\hline topic1196 & 0.00012402 & 0.00010477 & $2.31557 \mathrm{e}+06$ & $1.5199 \mathrm{e}-12$ & Reject \\
\hline topic1197 & 0.00013997 & $7.4455 \mathrm{e}-05$ & $1.16532 \mathrm{e}+06$ & $1.5305 \mathrm{e}-233$ & Reject \\
\hline topic1198 & 0.0002451 & 0.00017813 & $1.96168 \mathrm{e}+06$ & $1.9466 \mathrm{e}-50$ & Reject \\
\hline topic1199 & 0.00011937 & 0.00013965 & $2.34023 \mathrm{e}+06$ & 7.6919e-11 & Reject \\
\hline topic1200 & 0.00011757 & 0.00011411 & $2.56151 \mathrm{e}+06$ & 0.064518 & Accept \\
\hline topic1201 & $6.3938 \mathrm{e}-05$ & $1.1869 \mathrm{e}-05$ & $1.01057 \mathrm{e}+06$ & $6.2349 \mathrm{e}-282$ & Reject \\
\hline
\end{tabular}


Table A1 - Continued from previous page

\begin{tabular}{|c|c|c|c|c|c|}
\hline Feature & EN median & FL median & u-value & p-value & $\mathbf{H}_{0}$ \\
\hline topic1202 & 0.00013881 & $7.9415 \mathrm{e}-05$ & $1.87852 \mathrm{e}+06$ & $5.5476 \mathrm{e}-61$ & Reject \\
\hline topic1203 & 0.00012638 & $6.1028 \mathrm{e}-05$ & $1.84328 \mathrm{e}+06$ & $1.2677 \mathrm{e}-68$ & Reject \\
\hline topic1204 & $8.7099 \mathrm{e}-05$ & $6.2438 \mathrm{e}-05$ & $2.13322 \mathrm{e}+06$ & $1.1706 \mathrm{e}-28$ & Reject \\
\hline topic1205 & $7.4433 \mathrm{e}-05$ & $4.7263 \mathrm{e}-05$ & $1.92747 \mathrm{e}+06$ & $6.2515 \mathrm{e}-55$ & Reject \\
\hline topic1206 & $3.5903 \mathrm{e}-05$ & $2.0012 \mathrm{e}-05$ & $1.64065 \mathrm{e}+06$ & 2.8173e-107 & Reject \\
\hline topic1207 & $7.9324 \mathrm{e}-05$ & $6.6238 \mathrm{e}-05$ & $2.27086 \mathrm{e}+06$ & $7.628 \mathrm{e}-16$ & Reject \\
\hline topic1208 & $5.2197 \mathrm{e}-05$ & $3.8235 \mathrm{e}-05$ & $2.18346 \mathrm{e}+06$ & $2.0739 \mathrm{e}-23$ & Reject \\
\hline topic1209 & 0.00011072 & $9.5285 \mathrm{e}-05$ & $2.26648 \mathrm{e}+06$ & $2.8721 \mathrm{e}-16$ & Reject \\
\hline topic1210 & $6.9061 \mathrm{e}-05$ & $7.7365 \mathrm{e}-05$ & $2.48439 \mathrm{e}+06$ & 0.00078252 & Reject \\
\hline topic1211 & $9.0853 \mathrm{e}-05$ & $8.675 \mathrm{e}-05$ & $2.48989 \mathrm{e}+06$ & 0.0010869 & Reject \\
\hline topic1212 & $9.215 \mathrm{e}-05$ & $8.1841 \mathrm{e}-05$ & $2.38351 \mathrm{e}+06$ & $2.0586 \mathrm{e}-08$ & Reject \\
\hline topic1213 & $9.5579 \mathrm{e}-05$ & $4.32 \mathrm{e}-05$ & $1.19239 \mathrm{e}+06$ & $4.6343 \mathrm{e}-225$ & Reject \\
\hline topic1214 & $8.5607 \mathrm{e}-05$ & 0.00010562 & $2.276 \mathrm{e}+06$ & $1.6082 \mathrm{e}-15$ & Reject \\
\hline topic1215 & 0.00011136 & $5.3411 \mathrm{e}-05$ & $1.58756 \mathrm{e}+06$ & $1.0591 \mathrm{e}-118$ & Reject \\
\hline topic1216 & 0.00016128 & 0.00012557 & $2.12825 \mathrm{e}+06$ & $3.4704 \mathrm{e}-29$ & Reject \\
\hline topic1217 & 0.00015268 & 0.00011229 & $2.1899 \mathrm{e}+06$ & $8.7992 \mathrm{e}-23$ & Reject \\
\hline topic1218 & 0.00014927 & $7.5759 \mathrm{e}-05$ & $1.46211 \mathrm{e}+06$ & $3.1363 \mathrm{e}-148$ & Reject \\
\hline topic1219 & $6.0699 \mathrm{e}-05$ & $5.1877 \mathrm{e}-05$ & $2.3381 \mathrm{e}+06$ & $4.7842 \mathrm{e}-11$ & Reject \\
\hline topic1220 & $8.6303 \mathrm{e}-05$ & $5.0828 \mathrm{e}-05$ & $1.40607 \mathrm{e}+06$ & $4.3369 \mathrm{e}-164$ & Reject \\
\hline topic1221 & 0.00015449 & 0.00011625 & $2.04893 \mathrm{e}+06$ & $2.2757 \mathrm{e}-38$ & Reject \\
\hline topic1222 & $1.5203 \mathrm{e}-05$ & $6.5448 \mathrm{e}-05$ & $1.69641 \mathrm{e}+06$ & $1.6173 \mathrm{e}-82$ & Reject \\
\hline topic1223 & $9.7639 \mathrm{e}-05$ & $7.6764 \mathrm{e}-05$ & $2.32754 \mathrm{e}+06$ & $1.3682 \mathrm{e}-11$ & Reject \\
\hline topic1224 & $7.2295 \mathrm{e}-05$ & $6.8082 \mathrm{e}-05$ & $2.48116 \mathrm{e}+06$ & 0.00051262 & Reject \\
\hline topic1225 & 0.00013953 & $4.9101 \mathrm{e}-05$ & $1.24175 \mathrm{e}+06$ & $3.1718 \mathrm{e}-207$ & Reject \\
\hline topic1226 & $7.0655 \mathrm{e}-05$ & $3.1274 \mathrm{e}-05$ & $1.78349 \mathrm{e}+06$ & $5.1913 \mathrm{e}-79$ & Reject \\
\hline topic1227 & $6.5811 \mathrm{e}-06$ & $2.2473 \mathrm{e}-06$ & $2.20936 \mathrm{e}+06$ & $8.5519 \mathrm{e}-14$ & Reject \\
\hline topic1228 & 0.00013112 & $9.6094 \mathrm{e}-05$ & $2.04515 \mathrm{e}+06$ & 7.3863e-39 & Reject \\
\hline topic1229 & 0.00016003 & 0.00014854 & $2.50271 \mathrm{e}+06$ & 0.0043394 & Reject \\
\hline topic1230 & $5.0474 \mathrm{e}-05$ & $3.9667 \mathrm{e}-05$ & $2.35051 \mathrm{e}+06$ & $3.9341 \mathrm{e}-10$ & Reject \\
\hline topic1231 & 0.00010469 & 0.0001043 & $2.58695 \mathrm{e}+06$ & 0.17082 & Accept \\
\hline topic1232 & 0.00015155 & 0.00010038 & $2.04424 \mathrm{e}+06$ & 7.7992e-39 & Reject \\
\hline topic1233 & 0.00013464 & 0.00012022 & $2.38903 \mathrm{e}+06$ & $4.7029 \mathrm{e}-08$ & Reject \\
\hline topic1234 & 0.00013759 & $6.9491 \mathrm{e}-05$ & $2.16829 \mathrm{e}+06$ & $4.1931 \mathrm{e}-25$ & Reject \\
\hline topic1235 & $4.7487 \mathrm{e}-05$ & $3.5725 \mathrm{e}-05$ & $2.2371 \mathrm{e}+06$ & $1.323 \mathrm{e}-18$ & Reject \\
\hline topic1236 & 0.00010121 & $7.4872 \mathrm{e}-05$ & $2.07984 \mathrm{e}+06$ & $1.7305 \mathrm{e}-34$ & Reject \\
\hline topic1237 & $8.922 \mathrm{e}-05$ & $6.6844 \mathrm{e}-05$ & $2.30482 \mathrm{e}+06$ & $5.2146 \mathrm{e}-13$ & Reject \\
\hline topic1238 & $6.9232 \mathrm{e}-05$ & $6.2788 \mathrm{e}-05$ & $2.52705 \mathrm{e}+06$ & 0.011122 & Reject \\
\hline topic1239 & $4.6595 \mathrm{e}-05$ & $6.9789 \mathrm{e}-06$ & $1.04606 \mathrm{e}+06$ & $5.1532 \mathrm{e}-265$ & Reject \\
\hline topic1240 & $8.0628 \mathrm{e}-05$ & $3.7336 \mathrm{e}-05$ & $1.77842 \mathrm{e}+06$ & $3.0338 \mathrm{e}-80$ & Reject \\
\hline topic1241 & 0.00013833 & 0.00015957 & $2.33734 \mathrm{e}+06$ & $6.864 \mathrm{e}-11$ & Reject \\
\hline topic1242 & 0.00011214 & $8.3461 \mathrm{e}-05$ & $1.90254 \mathrm{e}+06$ & $6.1299 \mathrm{e}-59$ & Reject \\
\hline topic1243 & 0.00010966 & 0.00016156 & $2.28515 \mathrm{e}+06$ & $8.0597 \mathrm{e}-15$ & Reject \\
\hline
\end{tabular}


Table A1 - Continued from previous page

\begin{tabular}{|c|c|c|c|c|c|}
\hline Feature & EN median & FL median & u-value & p-value & $\mathrm{H}_{0}$ \\
\hline topic1244 & $6.1741 \mathrm{e}-05$ & 0.0001202 & $2.00399 \mathrm{e}+06$ & $4.094 \mathrm{e}-44$ & Reject \\
\hline topic1245 & $5.5524 \mathrm{e}-05$ & $4.3024 \mathrm{e}-05$ & $2.13251 \mathrm{e}+06$ & $1.005 \mathrm{e}-28$ & Reject \\
\hline topic1246 & $3.5036 \mathrm{e}-05$ & $2.1017 \mathrm{e}-05$ & $1.86344 \mathrm{e}+06$ & $6.005 \mathrm{e}-65$ & Reject \\
\hline topic1247 & 0.00013882 & $7.0201 \mathrm{e}-05$ & $1.53857 \mathrm{e}+06$ & $2.208 \mathrm{e}-130$ & Reject \\
\hline topic1248 & 0.00012713 & 7.3333e-05 & $1.71689 \mathrm{e}+06$ & $8.2685 \mathrm{e}-92$ & Reject \\
\hline topic1249 & 0.00011293 & $9.9578 \mathrm{e}-05$ & $2.38854 \mathrm{e}+06$ & $4.4293 \mathrm{e}-08$ & Reject \\
\hline topic1250 & 0.00013559 & $8.8673 \mathrm{e}-05$ & $1.50412 \mathrm{e}+06$ & $5.2881 \mathrm{e}-139$ & Reject \\
\hline topic1251 & 0.00012616 & 0.00010383 & $2.2141 \mathrm{e}+06$ & $1.5574 \mathrm{e}-20$ & Reject \\
\hline topic & $2.1863 \mathrm{e}-05$ & $2.2991 \mathrm{e}-05$ & $2.56902 \mathrm{e}+06$ & 0.20702 & Accept \\
\hline topic1253 & 0.0001621 & 0.00011275 & $2.37446 \mathrm{e}+06$ & $5.1052 \mathrm{e}-08$ & Reject \\
\hline topic1254 & $8.9253 \mathrm{e}-05$ & $3.4967 \mathrm{e}-05$ & $1.46826 \mathrm{e}+06$ & $7.3115 \mathrm{e}-148$ & Reject \\
\hline topi & 0.00013652 & 0.00011456 & $2.42414 \mathrm{e}+06$ & $2.9532 \mathrm{e}-06$ & Reject \\
\hline topic1256 & $8.249 \mathrm{e}-05$ & $4 e-05$ & $1.89229 \mathrm{e}+06$ & $2.7434 \mathrm{e}-56$ & Reject \\
\hline topi & $6.9299 \mathrm{e}-05$ & e-05 & $2.53689 \mathrm{e}+06$ & 0.019377 & Reject \\
\hline & 6.26 & & $2.62248 \mathrm{e}+06$ & & \\
\hline topic1259 & 0.00011333 & $8.4946 \mathrm{e}-05$ & $1.85231 \mathrm{e}+06$ & $1.4787 \mathrm{e}-67$ & Reject \\
\hline topi & $6.7268 \mathrm{e}-05$ & le-05 & $2.39288 \mathrm{e}+06$ & $1.2734 \mathrm{e}-07$ & Reject \\
\hline topic1261 & $5.3309 \mathrm{e}-05$ & $4 \mathrm{e}-05$ & $2.23163 \mathrm{e}+06$ & $4.4665 \mathrm{e}-19$ & Reject \\
\hline topi & $2 e-05$ & $e-05$ & $1.74434 \mathrm{e}+06$ & $1.1432 \mathrm{e}-86$ & Reject \\
\hline & & & & & \\
\hline topic1264 & $9.305 \mathrm{e}-05$ & $6.3353 \mathrm{e}-05$ & $2.09029 \mathrm{e}+06$ & $1.7927 \mathrm{e}-33$ & Reject \\
\hline topic1265 & $9.6259 \mathrm{e}-05$ & $1 \mathrm{e}-05$ & $1.97244 \mathrm{e}+06$ & $9.4267 \mathrm{e}-49$ & Reject \\
\hline topic1266 & $7.6069 \mathrm{e}-05$ & e-05 & $2.19145 \mathrm{e}+06$ & $1.2375 \mathrm{e}-22$ & Reject \\
\hline topic1267 & 0.0001443 & 0.00 & $2.40468 \mathrm{e}+06$ & $3.8855 \mathrm{e}-07$ & Reject \\
\hline topic1268 & $9.5796 \mathrm{e}-05$ & $5 e-05$ & $2.07331 \mathrm{e}+06$ & $1.6769 \mathrm{e}-35$ & Reject \\
\hline 1269 & $8.1824 \mathrm{e}-05$ & $1 \mathrm{e}-05$ & $2.29915 \mathrm{e}+06$ & $1.0521 \mathrm{e}-13$ & Reject \\
\hline 270 & $1.2322 \mathrm{e}-05$ & $9 \mathrm{e}-05$ & $1.96982 \mathrm{e}+06$ & $1.7371 \mathrm{e}-45$ & Reject \\
\hline & 0.00010098 & $5 e-05$ & $1.80574 \mathrm{e}+06$ & $8.0083 \mathrm{e}-75$ & Reject \\
\hline 272 & 0.00012409 & $9.6992 \mathrm{e}-05$ & $2.26836 \mathrm{e}+06$ & $8.6983 \mathrm{e}-16$ & Reject \\
\hline topic1273 & 0.00010596 & $4 \mathrm{e}-05$ & $2.27313 \mathrm{e}+06$ & $1.415 \mathrm{e}-15$ & Reject \\
\hline topic1274 & $1.1455 \mathrm{e}-05$ & $1.2369 \mathrm{e}-06$ & $1.40097 \mathrm{e}+06$ & $.7088 \mathrm{e}-159$ & Reject \\
\hline topic1275 & 0.00010328 & 0.00010772 & $2.54531 \mathrm{e}+06$ & 0.031943 & Reject \\
\hline & $6059 \mathrm{e}-05$ & $9.01 \mathrm{e}-06$ & $1.40986 \mathrm{e}+06$ & $1.0719 \mathrm{e}-162$ & Reject \\
\hline 1277 & $.0606 \mathrm{e}-05$ & $3.9541 \mathrm{e}-05$ & $1.8405 \mathrm{e}+06$ & $1.4678 \mathrm{e}-69$ & Reject \\
\hline topic1278 & $8.0676 \mathrm{e}-05$ & $8.5481 \mathrm{e}-05$ & $2.53694 \mathrm{e}+06$ & 0.019426 & Reject \\
\hline & $2.3805 \mathrm{e}-05$ & $8.0071 \mathrm{e}-06$ & $1.76413 \mathrm{e}+06$ & $3.2372 \mathrm{e}-82$ & Reject \\
\hline topic1280 & 0.0001223 & $8.241 \mathrm{e}-05$ & $2.05555 \mathrm{e}+06$ & $1.1456 \mathrm{e}-37$ & Reject \\
\hline topic1281 & 0.00018679 & 0.00012571 & $1.89019 \mathrm{e}+06$ & $1.3226 \mathrm{e}-60$ & Reject \\
\hline topic1282 & 0.00010989 & $9.8099 \mathrm{e}-05$ & $2.51399 \mathrm{e}+06$ & 0.0099822 & Reject \\
\hline topic1283 & 0.00011262 & 0.00013376 & $2.33398 \mathrm{e}+06$ & $2.2001 \mathrm{e}-11$ & Reject \\
\hline & $7.4319 \mathrm{e}-05$ & $4.4345 \mathrm{e}-05$ & $2.07677 \mathrm{e}+06$ & $1.419 \mathrm{e}-33$ & Reject \\
\hline topic1285 & $8.1661 \mathrm{e}-05$ & $7.6601 \mathrm{e}-05$ & $2.53051 \mathrm{e}+06$ & 0.015333 & Reject \\
\hline
\end{tabular}


Table A1 - Continued from previous page

\begin{tabular}{|c|c|c|c|c|c|}
\hline Feature & EN median & FL median & u-value & p-value & $\mathbf{H}_{0}$ \\
\hline topic1286 & 0.00011282 & $8.2317 \mathrm{e}-05$ & $2.21916 \mathrm{e}+06$ & $3.4982 \mathrm{e}-20$ & Reject \\
\hline topic1287 & $6.9052 \mathrm{e}-05$ & $1.6593 \mathrm{e}-05$ & $1.11561 \mathrm{e}+06$ & $9.0104 \mathrm{e}-248$ & Reject \\
\hline topic1288 & 0.00021018 & 0.00017546 & $2.24591 \mathrm{e}+06$ & $7.2355 \mathrm{e}-18$ & Reject \\
\hline topic1289 & $8.5674 \mathrm{e}-05$ & $3.9938 \mathrm{e}-05$ & $1.62953 \mathrm{e}+06$ & $4.91 \mathrm{e}-110$ & Reject \\
\hline topic1290 & 0.00015024 & $5.6301 \mathrm{e}-05$ & $1.37228 \mathrm{e}+06$ & $2.5148 \mathrm{e}-172$ & Reject \\
\hline topic1291 & $7.0602 \mathrm{e}-05$ & $4.1559 \mathrm{e}-05$ & $2.09208 \mathrm{e}+06$ & 2.3691e-32 & Reject \\
\hline topic1292 & $3.2636 \mathrm{e}-05$ & $2.2125 \mathrm{e}-05$ & $1.77951 \mathrm{e}+06$ & $1.5356 \mathrm{e}-79$ & Reject \\
\hline topic1293 & 0.00011845 & 0.00010118 & $2.17849 \mathrm{e}+06$ & $5.4865 \mathrm{e}-24$ & Reject \\
\hline topic1294 & 0.00012396 & $8.5991 \mathrm{e}-05$ & $1.75407 \mathrm{e}+06$ & $5.676 \mathrm{e}-85$ & Reject \\
\hline topic1295 & 0.00017166 & 0.00010182 & $1.88585 \mathrm{e}+06$ & $2.6771 \mathrm{e}-61$ & Reject \\
\hline topic1296 & $7.2183 \mathrm{e}-05$ & $7.958 \mathrm{e}-05$ & $2.44421 \mathrm{e}+06$ & $2.0002 \mathrm{e}-05$ & Reject \\
\hline topic1297 & $5.4464 \mathrm{e}-05$ & $4.8187 \mathrm{e}-05$ & $2.48617 \mathrm{e}+06$ & 0.00075173 & Reject \\
\hline topic1298 & $1.888 \mathrm{e}-05$ & $3.6613 \mathrm{e}-06$ & $1.31501 \mathrm{e}+06$ & $2.3035 \mathrm{e}-187$ & Reject \\
\hline topic1299 & $4.2908 \mathrm{e}-05$ & $3.5366 \mathrm{e}-05$ & $2.26806 \mathrm{e}+06$ & $5.5802 \mathrm{e}-16$ & Reject \\
\hline topic1300 & 0.00013683 & $9.2498 \mathrm{e}-05$ & $1.74326 \mathrm{e}+06$ & $3.451 \mathrm{e}-87$ & Reject \\
\hline topic1301 & 0.00013009 & 0.00015696 & $2.41393 \mathrm{e}+06$ & $9.6948 \mathrm{e}-07$ & Reject \\
\hline topic1302 & 0.00012156 & $7.1692 \mathrm{e}-05$ & $1.90297 \mathrm{e}+06$ & $3.8792 \mathrm{e}-58$ & Reject \\
\hline topic1303 & $6.7543 \mathrm{e}-06$ & $1.7361 \mathrm{e}-05$ & $1.93744 \mathrm{e}+06$ & $2.2116 \mathrm{e}-50$ & Reject \\
\hline topic1304 & 0.00017322 & 0.00011306 & $1.94412 \mathrm{e}+06$ & $6.8216 \mathrm{e}-52$ & Reject \\
\hline topic1305 & 0.00019723 & 0.00016159 & $2.20534 \mathrm{e}+06$ & $1.9752 \mathrm{e}-21$ & Reject \\
\hline topic1306 & $8.1382 \mathrm{e}-05$ & $4.6563 \mathrm{e}-05$ & $1.91727 \mathrm{e}+06$ & $4.4521 \mathrm{e}-57$ & Reject \\
\hline topic1307 & $5.2867 \mathrm{e}-05$ & $3.4636 \mathrm{e}-05$ & $2.18433 \mathrm{e}+06$ & $1.2773 \mathrm{e}-22$ & Reject \\
\hline topic1308 & 0.00011506 & 0.00013325 & $2.34772 \mathrm{e}+06$ & $1.6535 \mathrm{e}-10$ & Reject \\
\hline topic1309 & 0.00016338 & 0.00020156 & $2.20753 \mathrm{e}+06$ & $2.5154 \mathrm{e}-21$ & Reject \\
\hline topic1310 & 0.0001627 & 0.00020868 & $2.31826 \mathrm{e}+06$ & 3.8856e-12 & Reject \\
\hline topic1311 & 0.00013939 & $7.1129 \mathrm{e}-05$ & $1.8758 \mathrm{e}+06$ & $3.2439 \mathrm{e}-63$ & Reject \\
\hline topic1312 & 0.00015919 & 0.00011734 & $2.07209 \mathrm{e}+06$ & $4.6207 \mathrm{e}-35$ & Reject \\
\hline topic1313 & 0.00010452 & $6.9979 \mathrm{e}-05$ & $2.18176 \mathrm{e}+06$ & $1.105 \mathrm{e}-22$ & Reject \\
\hline topic1314 & 0.00013173 & $9.1185 \mathrm{e}-05$ & $1.73225 \mathrm{e}+06$ & $3.8615 \mathrm{e}-89$ & Reject \\
\hline topic1315 & $1.2046 \mathrm{e}-05$ & $2.9048 \mathrm{e}-05$ & $1.69395 \mathrm{e}+06$ & $2.1927 \mathrm{e}-77$ & Reject \\
\hline topic1316 & $9.1446 \mathrm{e}-05$ & $9.0365 \mathrm{e}-05$ & $2.55169 \mathrm{e}+06$ & 0.04817 & Reject \\
\hline topic1317 & 0.0001346 & 0.00010641 & $2.30661 \mathrm{e}+06$ & $3.0132 \mathrm{e}-13$ & Reject \\
\hline topic1318 & $4.3319 \mathrm{e}-05$ & $2.8633 \mathrm{e}-05$ & $1.66114 \mathrm{e}+06$ & $1.1584 \mathrm{e}-103$ & Reject \\
\hline topic1319 & 0.00014556 & 0.00011614 & $2.38768 \mathrm{e}+06$ & $5.867 \mathrm{e}-08$ & Reject \\
\hline topic1320 & $2.5557 \mathrm{e}-05$ & $3.4161 \mathrm{e}-05$ & $2.30096 \mathrm{e}+06$ & $4.6648 \mathrm{e}-13$ & Reject \\
\hline topic1321 & 0.00013691 & $7.4499 \mathrm{e}-05$ & $1.93424 \mathrm{e}+06$ & $4.5456 \mathrm{e}-54$ & Reject \\
\hline topic1322 & 0.00012073 & $7.4729 \mathrm{e}-05$ & $2.15812 \mathrm{e}+06$ & $6.2083 \mathrm{e}-26$ & Reject \\
\hline topic1323 & $3.6289 \mathrm{e}-05$ & $9.3904 \mathrm{e}-06$ & $1.3383 \mathrm{e}+06$ & $2.7272 \mathrm{e}-182$ & Reject \\
\hline topic1324 & 0.00014524 & 0.00016304 & $2.36685 \mathrm{e}+06$ & $2.7325 \mathrm{e}-09$ & Reject \\
\hline topic1325 & 0.00011897 & 0.00013166 & $2.44376 \mathrm{e}+06$ & $1.9151 \mathrm{e}-05$ & Reject \\
\hline topic1326 & $3.1728 \mathrm{e}-05$ & $5.3963 \mathrm{e}-05$ & $2.03 \mathrm{e}+06$ & $7.9277 \mathrm{e}-40$ & Reject \\
\hline topic1327 & $6.2699 \mathrm{e}-08$ & $9.7696 \mathrm{e}-08$ & 895492 & 2.5491e-19 & Reject \\
\hline
\end{tabular}


Table A1 - Continued from previous page

\begin{tabular}{|c|c|c|c|c|c|}
\hline Feature & EN median & FL median & u-value & p-value & $\mathbf{H}_{0}$ \\
\hline topic1328 & 0.00010836 & 0.00014728 & $2.06441 \mathrm{e}+06$ & $1.8922 \mathrm{e}-36$ & Reject \\
\hline topic1329 & 0.00012857 & $8.0085 \mathrm{e}-05$ & $1.88271 \mathrm{e}+06$ & $6.8615 \mathrm{e}-61$ & Reject \\
\hline topic1330 & $7.6483 \mathrm{e}-05$ & $7.056 \mathrm{e}-05$ & $2.4875 \mathrm{e}+06$ & 0.00098325 & Reject \\
\hline topic1331 & $7.5555 \mathrm{e}-05$ & $5.6587 \mathrm{e}-05$ & $2.23619 \mathrm{e}+06$ & $1.6538 \mathrm{e}-18$ & Reject \\
\hline topic1332 & 0.00010177 & 0.00010347 & $2.61590 \mathrm{e}+06$ & 0.38 & Accept \\
\hline topic1333 & 0.00012337 & $9.2748 \mathrm{e}-05$ & $1.98669 \mathrm{e}+06$ & $1.3094 \mathrm{e}-46$ & Reject \\
\hline topic1334 & $4.1147 \mathrm{e}-05$ & $4.5362 \mathrm{e}-05$ & $2.59152 \mathrm{e}+06$ & 0.19791 & Accept \\
\hline topic1335 & $6.6412 \mathrm{e}-05$ & $6.3832 \mathrm{e}-05$ & $2.59054 \mathrm{e}+06$ & 0.19188 & Accept \\
\hline topic1336 & $6.3199 \mathrm{e}-05$ & $7.0921 \mathrm{e}-05$ & $2.51416 \mathrm{e}+06$ & 0.0076554 & Reject \\
\hline topic1337 & 0.00010719 & $6.0724 \mathrm{e}-05$ & $1.54976 \mathrm{e}+06$ & $5.924 \mathrm{e}-128$ & Reject \\
\hline topic1338 & 0.00013325 & 0.00010876 & $2.24035 \mathrm{e}+06$ & $2.5047 \mathrm{e}-18$ & Reject \\
\hline topic1339 & $2.2604 \mathrm{e}-05$ & $3.2662 \mathrm{e}-06$ & $1.78434 \mathrm{e}+06$ & $2.8452 \mathrm{e}-75$ & Reject \\
\hline topic1340 & 0.00013359 & 0.00011426 & $2.33589 \mathrm{e}+06$ & $4.7831 \mathrm{e}-11$ & Reject \\
\hline topic1341 & $5.2185 \mathrm{e}-05$ & $3.4359 \mathrm{e}-05$ & $2.02501 \mathrm{e}+06$ & $1.0662 \mathrm{e}-41$ & Reject \\
\hline topic1342 & 0.00012956 & $9.88 \mathrm{e}-05$ & $2.01723 \mathrm{e}+06$ & $2.4166 \mathrm{e}-42$ & Reject \\
\hline topic1343 & $3.4114 \mathrm{e}-05$ & $5.377 \mathrm{e}-06$ & 907218 & 0 & Reject \\
\hline topic1344 & 0.00011618 & $6.923 \mathrm{e}-05$ & $1.72476 \mathrm{e}+06$ & $1.8624 \mathrm{e}-90$ & Reject \\
\hline topic1345 & 0.00017116 & 0.00014329 & $2.17294 \mathrm{e}+06$ & $1.5192 \mathrm{e}-24$ & Reject \\
\hline topic1346 & 0.00010784 & $5.8033 \mathrm{e}-05$ & $1.43044 \mathrm{e}+06$ & $1.6678 \mathrm{e}-157$ & Reject \\
\hline topic1347 & 0.00010171 & $3.1691 \mathrm{e}-05$ & $1.32111 \mathrm{e}+06$ & $3.888 \mathrm{e}-183$ & Reject \\
\hline topic1348 & $8.2692 \mathrm{e}-05$ & $1.0215 \mathrm{e}-06$ & 786168 & 0 & Reject \\
\hline topic1349 & $8.4126 \mathrm{e}-05$ & $6.7605 \mathrm{e}-05$ & $2.30514 \mathrm{e}+06$ & $3.3831 \mathrm{e}-13$ & Reject \\
\hline topic1350 & 0.0001072 & $8.512 \mathrm{e}-05$ & $2.24096 \mathrm{e}+06$ & $6.2371 \mathrm{e}-18$ & Reject \\
\hline topic1351 & 0.00014789 & 0.00014086 & $2.49545 \mathrm{e}+06$ & 0.001788 & Reject \\
\hline topic1352 & $8.6383 \mathrm{e}-05$ & $5.8232 \mathrm{e}-05$ & $2.04553 \mathrm{e}+06$ & $8.4866 \mathrm{e}-39$ & Reject \\
\hline topic1353 & $6.1531 \mathrm{e}-05$ & $1.0309 \mathrm{e}-05$ & $1.07667 \mathrm{e}+06$ & $2.4587 \mathrm{e}-261$ & Reject \\
\hline topic1354 & 0.00011458 & $6.5747 \mathrm{e}-05$ & $1.67873 \mathrm{e}+06$ & $1.735 \mathrm{e}-99$ & Reject \\
\hline topic1355 & 0.00011475 & $7.8994 \mathrm{e}-05$ & $2.01475 \mathrm{e}+06$ & $1.9722 \mathrm{e}-42$ & Reject \\
\hline topic1356 & $6.0415 \mathrm{e}-05$ & $5.054 \mathrm{e}-05$ & $2.19235 \mathrm{e}+06$ & $9.4973 \mathrm{e}-23$ & Reject \\
\hline topic1357 & $3.314 \mathrm{e}-05$ & $2.1221 \mathrm{e}-05$ & $2.09393 \mathrm{e}+06$ & $4.649 \mathrm{e}-33$ & Reject \\
\hline topic1358 & 0.00014167 & 0.00012736 & $2.33175 \mathrm{e}+06$ & $2.5083 \mathrm{e}-11$ & Reject \\
\hline topic1359 & $8.7659 \mathrm{e}-05$ & $6.4673 \mathrm{e}-05$ & $2.14012 \mathrm{e}+06$ & $5.089 \mathrm{e}-28$ & Reject \\
\hline topic1360 & 0.00012704 & 0.00013 & $2.571 \mathrm{e}+06$ & 0.10023 & Accept \\
\hline topic1361 & 0.0001123 & $7.2484 \mathrm{e}-05$ & $2.00583 \mathrm{e}+06$ & $2.9678 \mathrm{e}-44$ & Reject \\
\hline topic1362 & 0.00012128 & $8.9868 \mathrm{e}-05$ & $2.01438 \mathrm{e}+06$ & $5.6732 \mathrm{e}-43$ & Reject \\
\hline topic1363 & 0.00011162 & $7.4966 \mathrm{e}-05$ & $1.87532 \mathrm{e}+06$ & $9.6754 \mathrm{e}-64$ & Reject \\
\hline topic1364 & 0.00018089 & $9.6079 \mathrm{e}-05$ & $1.70622 \mathrm{e}+06$ & 8.0221e-92 & Reject \\
\hline topic1365 & 0.0002418 & 0.00019953 & $2.10136 \mathrm{e}+06$ & $3.4256 \mathrm{e}-32$ & Reject \\
\hline topic1366 & 0.00011309 & $9.324 \mathrm{e}-05$ & $2.28574 \mathrm{e}+06$ & $1.5406 \mathrm{e}-14$ & Reject \\
\hline topic1367 & 0.00013181 & $9.2984 \mathrm{e}-05$ & $2.0469 \mathrm{e}+06$ & $1.624 \mathrm{e}-37$ & Reject \\
\hline topic1368 & $6.2492 \mathrm{e}-05$ & $4.7327 \mathrm{e}-05$ & $2.24224 \mathrm{e}+06$ & $1 \mathrm{e}-17$ & Reject \\
\hline topic1369 & 0.00012045 & $9.7932 \mathrm{e}-05$ & $2.02304 \mathrm{e}+06$ & $7.9451 \mathrm{e}-42$ & Reject \\
\hline
\end{tabular}


Table A1 - Continued from previous page

\begin{tabular}{|c|c|c|c|c|c|}
\hline Feature & EN median & FL median & u-value & p-value & $\mathrm{H}_{\mathbf{0}}$ \\
\hline topic1370 & 0.00010286 & $7.082 \mathrm{e}-05$ & $1.68627 \mathrm{e}+06$ & $4.1594 \mathrm{e}-98$ & Reject \\
\hline topic1371 & 0.0001286 & 0.00014125 & $2.52911 \mathrm{e}+06$ & 0.013404 & Reject \\
\hline topic1372 & $9.6727 \mathrm{e}-05$ & 7.6965e-05 & $2.02497 \mathrm{e}+06$ & $1.4214 \mathrm{e}-41$ & Reject \\
\hline topic1373 & 5.243 & $1.1335 \mathrm{e}-05$ & $1.12945 \mathrm{e}+06$ & $7.5446 \mathrm{e}-243$ & Reject \\
\hline topic1374 & 0.00018215 & 0.00017345 & $2.50049 \mathrm{e}+06$ & 0.0019998 & Reject \\
\hline topic1375 & $6.923 \mathrm{e}-05$ & $4.1255 \mathrm{e}-05$ & $1.91718 \mathrm{e}+06$ & $4.3643 \mathrm{e}-56$ & Reject \\
\hline topic1376 & $9.8963 \mathrm{e}-05$ & 0.00012614 & $2.23106 \mathrm{e}+06$ & $3.9863 \mathrm{e}-19$ & Reject \\
\hline topic1377 & $3.7623 \mathrm{e}-05$ & $2.2215 \mathrm{e}-05$ & $1.74628 \mathrm{e}+06$ & $2.8877 \mathrm{e}-86$ & Reject \\
\hline topic1378 & 0.00014705 & $6.5067 \mathrm{e}-05$ & $1.77823 \mathrm{e}+06$ & $1.1405 \mathrm{e}-78$ & Reject \\
\hline topic1379 & 0.00012067 & $7.1257 \mathrm{e}-05$ & $1.59008 \mathrm{e}+06$ & $3.5744 \mathrm{e}-118$ & Reject \\
\hline topic1380 & $9.9349 \mathrm{e}-05$ & 0.00013671 & $2.11499 \mathrm{e}+06$ & $1.9796 \mathrm{e}-30$ & Reject \\
\hline topic1381 & $4.1805 \mathrm{e}-05$ & $2.7552 \mathrm{e}-05$ & $1.81746 \mathrm{e}+06$ & $3.0404 \mathrm{e}-73$ & Reject \\
\hline topic1382 & 0.00012818 & 0.00014691 & $2.34874 \mathrm{e}+06$ & $1.9152 \mathrm{e}-10$ & Reject \\
\hline & 0.00013069 & 0.00012095 & $2.4354 \mathrm{e}+06$ & 8.3923e-06 & Reject \\
\hline top & 0.00010904 & $4 e-05$ & $2.09913 \mathrm{e}+06$ & & \\
\hline topic1385 & $8.0982 \mathrm{e}-06$ & $8.6633 \mathrm{e}-06$ & $2.50757 \mathrm{e}+06$ & 0.0077868 & Reject \\
\hline topic1386 & 0.00012759 & 0.00010252 & $2.24614 \mathrm{e}+06$ & $6.2718 \mathrm{e}-18$ & Reject \\
\hline topic1387 & $1.7207 \mathrm{e}-05$ & $5 e-05$ & $2.13963 \mathrm{e}+06$ & $7.4991 \mathrm{e}-28$ & Reject \\
\hline & 0.00010056 & 1434 & $2.43486 \mathrm{e}+06$ & $7.086 \mathrm{e}-06$ & ect \\
\hline & & & & & \\
\hline topic1390 & $6.8044 \mathrm{e}-05$ & $5.2252 \mathrm{e}-05$ & $2.38747 \mathrm{e}+06$ & $3.8798 \mathrm{e}-08$ & Reject \\
\hline topic1391 & $9.8835 \mathrm{e}-05$ & $6.0599 \mathrm{e}-05$ & $2.01069 \mathrm{e}+06$ & $6.169 \mathrm{e}-43$ & Reject \\
\hline topic1392 & 0.00011926 & 8e-05 & $2.1528 \mathrm{e}+06$ & $2.8243 \mathrm{e}-26$ & Reject \\
\hline topic1393 & 5e-05 & $4 \mathrm{e}-05$ & $2.36181 \mathrm{e}+06$ & $1.1903 \mathrm{e}-09$ & Reject \\
\hline topic1394 & 0.00015255 & 0.00010318 & $1.98145 \mathrm{e}+06$ & $5.7452 \mathrm{e}-47$ & Reject \\
\hline topic1395 & $8.4555 \mathrm{e}-05$ & $8.103 \mathrm{e}-05$ & $2.56563 \mathrm{e}+06$ & 0.080753 & Accept \\
\hline 1396 & $5.4009 \mathrm{e}-05$ & $4.2961 \mathrm{e}-05$ & $2.39919 \mathrm{e}+06$ & $4.3337 \mathrm{e}-07$ & Reject \\
\hline & 0.0001083 & $9 e-05$ & $1.26076 \mathrm{e}+06$ & $1.4497 \mathrm{e}-204$ & Reject \\
\hline topic1398 & $2.4179 \mathrm{e}-05$ & $1.1758 \mathrm{e}-05$ & $1.72548 \mathrm{e}+06$ & $3.8501 \mathrm{e}-90$ & Reject \\
\hline topic1399 & $8.0988 \mathrm{e}-05$ & $9.5838 \mathrm{e}-05$ & $2.4535 \mathrm{e}+06$ & $5.2865 \mathrm{e}-05$ & Reject \\
\hline 400 & 0.0001526 & 0.00019454 & $2.13479 \mathrm{e}+06$ & $2.8788 \mathrm{e}-28$ & Reject \\
\hline topic1401 & $9.2337 \mathrm{e}-05$ & $8.0758 \mathrm{e}-05$ & $2.207 \mathrm{e}+06$ & $2.8085 \mathrm{e}-21$ & Reject \\
\hline & 0.00012128 & 0.00010161 & $2.1932 \mathrm{e}+06$ & $1.4426 \mathrm{e}-22$ & Reject \\
\hline 1403 & $3.9849 \mathrm{e}-05$ & $1.8527 \mathrm{e}-05$ & $1.49218 \mathrm{e}+06$ & $5.8275 \mathrm{e}-141$ & Reject \\
\hline topic1404 & $7.9301 \mathrm{e}-05$ & $8.7175 \mathrm{e}-05$ & $2.5021 \mathrm{e}+06$ & 0.0035519 & Reject \\
\hline & $8.4449 \mathrm{e}-05$ & $4.0337 \mathrm{e}-05$ & $1.62391 \mathrm{e}+06$ & $1.0826 \mathrm{e}-110$ & Reject \\
\hline topic1406 & 0.00012385 & 0.0001424 & $2.43337 \mathrm{e}+06$ & $6.8367 \mathrm{e}-06$ & Reject \\
\hline topic1407 & 0.00011895 & $8.4642 \mathrm{e}-05$ & $2.01373 \mathrm{e}+06$ & $1.483 \mathrm{e}-42$ & Reject \\
\hline topic1408 & $3.5646 \mathrm{e}-05$ & $1.611 \mathrm{e}-05$ & $1.3935 \mathrm{e}+06$ & $3.1898 \mathrm{e}-167$ & Reject \\
\hline topic1409 & 0.00014336 & $7.1308 \mathrm{e}-05$ & $1.43528 \mathrm{e}+06$ & $1.1186 \mathrm{e}-155$ & Reject \\
\hline topic1410 & $3.3077 \mathrm{e}-05$ & $1.8479 \mathrm{e}-05$ & $1.66185 \mathrm{e}+06$ & $1.632 \mathrm{e}-103$ & Reject \\
\hline topic1411 & 0.0001506 & 0.00013819 & $2.44508 \mathrm{e}+06$ & $2.176 \mathrm{e}-05$ & Reject \\
\hline
\end{tabular}


Table A1 - Continued from previous page

\begin{tabular}{|c|c|c|c|c|c|}
\hline Feature & EN median & FL median & u-value & p-value & $\overline{\mathbf{H}_{0}}$ \\
\hline topic1412 & 0.00011498 & 0.00014672 & $2.43739 \mathrm{e}+06$ & $1.5729 \mathrm{e}-05$ & Reject \\
\hline topic1413 & 0.00015678 & 0.00014997 & $2.51038 \mathrm{e}+06$ & 0.0039338 & Reject \\
\hline topic1414 & 0.00013237 & $9.7066 \mathrm{e}-05$ & $2.15411 \mathrm{e}+06$ & $3.0802 \mathrm{e}-26$ & Reject \\
\hline topic1415 & $4.8294 \mathrm{e}-05$ & $3.347 \mathrm{e}-05$ & $1.8957 \mathrm{e}+06$ & $3.6151 \mathrm{e}-60$ & Reject \\
\hline topic1416 & $3.8206 \mathrm{e}-05$ & $4.5047 \mathrm{e}-05$ & $2.48737 \mathrm{e}+06$ & 0.00083021 & Reject \\
\hline topic1417 & 0.00012094 & $8.9979 \mathrm{e}-05$ & $1.87833 \mathrm{e}+06$ & $6.0725 \mathrm{e}-63$ & Reject \\
\hline topic1418 & 0.00017824 & 0.00010326 & $1.95348 \mathrm{e}+06$ & $1.1535 \mathrm{e}-50$ & Reject \\
\hline topic1419 & $6.3846 \mathrm{e}-05$ & $2.6245 \mathrm{e}-05$ & $1.35795 \mathrm{e}+06$ & $1.2242 \mathrm{e}-176$ & Reject \\
\hline topic1420 & $8.969 \mathrm{e}-05$ & $7.2178 \mathrm{e}-05$ & $2.3615 \mathrm{e}+06$ & $2.055 \mathrm{e}-09$ & Reject \\
\hline topic1421 & 0.00014939 & 0.00021788 & $1.84776 \mathrm{e}+06$ & $2.524 \mathrm{e}-68$ & Reject \\
\hline topic1422 & $3.9891 \mathrm{e}-05$ & $4.0805 \mathrm{e}-05$ & $2.62232 \mathrm{e}+06$ & 0.47512 & Accept \\
\hline topic1423 & 0.00013513 & 0.00013019 & $2.57788 \mathrm{e}+06$ & 0.15304 & Accept \\
\hline topic1424 & $8.1884 \mathrm{e}-05$ & $2.8368 \mathrm{e}-05$ & $2.05714 \mathrm{e}+06$ & $7.0138 \mathrm{e}-37$ & Reject \\
\hline topic1425 & $3.7169 \mathrm{e}-05$ & $3.1573 \mathrm{e}-05$ & $2.21688 \mathrm{e}+06$ & $2.7719 \mathrm{e}-20$ & Reject \\
\hline topic1426 & $1.9378 \mathrm{e}-05$ & $1.8244 \mathrm{e}-05$ & $2.46812 \mathrm{e}+06$ & 0.001769 & Reject \\
\hline topic1427 & 0.0001659 & 0.00016761 & $2.60352 \mathrm{e}+06$ & 0.28046 & Accept \\
\hline topic1428 & $2.1888 \mathrm{e}-05$ & $1.0941 \mathrm{e}-05$ & $1.94812 \mathrm{e}+06$ & $3.273 \mathrm{e}-50$ & Reject \\
\hline topic1429 & 0.00012095 & 0.00010518 & $2.34767 \mathrm{e}+06$ & $1.8967 \mathrm{e}-10$ & Reject \\
\hline topic1430 & 0.00010905 & $5.7376 \mathrm{e}-05$ & $1.76867 \mathrm{e}+06$ & $4.7423 \mathrm{e}-81$ & Reject \\
\hline topic1431 & $9.7969 \mathrm{e}-05$ & $7.4605 \mathrm{e}-05$ & $2.3131 \mathrm{e}+06$ & $1.0259 \mathrm{e}-12$ & Reject \\
\hline topic1432 & 0.00013804 & $7.216 \mathrm{e}-05$ & $1.45068 \mathrm{e}+06$ & $4.2146 \mathrm{e}-152$ & Reject \\
\hline topic1433 & 0.00014619 & $7.5011 \mathrm{e}-05$ & $1.65112 \mathrm{e}+06$ & $1.607 \mathrm{e}-104$ & Reject \\
\hline topic1434 & $6.5514 \mathrm{e}-05$ & $4.8598 \mathrm{e}-05$ & $1.98556 \mathrm{e}+06$ & $6.6409 \mathrm{e}-47$ & Reject \\
\hline topic1435 & $9.1648 \mathrm{e}-05$ & $8.6603 \mathrm{e}-05$ & $2.55254 \mathrm{e}+06$ & 0.042894 & Reject \\
\hline topic1436 & 0.00012463 & $7.0775 \mathrm{e}-05$ & $1.66429 \mathrm{e}+06$ & $5.8159 \mathrm{e}-102$ & Reject \\
\hline topic1437 & 0.00014747 & 0.00012219 & $2.38271 \mathrm{e}+06$ & $6.4043 \mathrm{e}-08$ & Reject \\
\hline topic1438 & $6.5918 \mathrm{e}-05$ & $1.9408 \mathrm{e}-05$ & $1.21870 \mathrm{e}+06$ & $4.3517 \mathrm{e}-216$ & Reject \\
\hline topic1439 & $9.4979 \mathrm{e}-06$ & $1.3177 \mathrm{e}-05$ & $2.282 \mathrm{e}+06$ & $8.0657 \mathrm{e}-15$ & Reject \\
\hline topic1440 & $7.7781 \mathrm{e}-05$ & $3.8841 \mathrm{e}-05$ & $1.78138 \mathrm{e}+06$ & $2.0641 \mathrm{e}-79$ & Reject \\
\hline topic1441 & 0.0001004 & $9.1743 \mathrm{e}-05$ & $2.49279 \mathrm{e}+06$ & 0.0014759 & Reject \\
\hline topic1442 & 0.00012961 & 0.00010106 & $2.01139 \mathrm{e}+06$ & $3.0672 \mathrm{e}-43$ & Reject \\
\hline topic1443 & 0.00012624 & $9.5973 \mathrm{e}-05$ & $2.09725 \mathrm{e}+06$ & $1.478 \mathrm{e}-32$ & Reject \\
\hline topic1444 & 0.00010659 & $8.8826 \mathrm{e}-05$ & $2.40481 \mathrm{e}+06$ & $4.4867 \mathrm{e}-07$ & Reject \\
\hline topic1445 & 0.00010551 & $8.9062 \mathrm{e}-05$ & $2.49555 \mathrm{e}+06$ & 0.001788 & Reject \\
\hline topic1446 & $5.8873 \mathrm{e}-05$ & $2.6713 \mathrm{e}-05$ & $1.55572 \mathrm{e}+06$ & $2.2502 \mathrm{e}-126$ & Reject \\
\hline topic1447 & $2.6745 \mathrm{e}-05$ & $2.9209 \mathrm{e}-06$ & $1.24173 \mathrm{e}+06$ & 2.1177e-209 & Reject \\
\hline topic1448 & 0.0001668 & 0.00017383 & $2.53844 \mathrm{e}+06$ & 0.02384 & Reject \\
\hline topic1449 & $2.8785 \mathrm{e}-05$ & $4.518 \mathrm{e}-06$ & $1.08716 \mathrm{e}+06$ & $1.7951 \mathrm{e}-256$ & Reject \\
\hline topic1450 & 0.00011389 & 0.00010411 & $2.46023 \mathrm{e}+06$ & $8.7596 \mathrm{e}-05$ & Reject \\
\hline topic1451 & $9.7953 \mathrm{e}-05$ & $8.2953 \mathrm{e}-05$ & $2.28382 \mathrm{e}+06$ & $6.3866 \mathrm{e}-15$ & Reject \\
\hline topic1452 & 0.00015819 & $7.4406 \mathrm{e}-05$ & $1.60089 \mathrm{e}+06$ & $3.1277 \mathrm{e}-114$ & Reject \\
\hline topic1453 & $5.4128 \mathrm{e}-06$ & $6.9308 \mathrm{e}-06$ & $2.60307 \mathrm{e}+06$ & 0.28604 & Accept \\
\hline
\end{tabular}


Table A1 - Continued from previous page

\begin{tabular}{|c|c|c|c|c|c|}
\hline Feature & EN median & FL median & u-value & p-value & $\mathbf{H}_{0}$ \\
\hline topic1454 & 0.00011483 & $8.1853 \mathrm{e}-05$ & $1.99482 \mathrm{e}+06$ & $9.3427 \mathrm{e}-46$ & Reject \\
\hline topic1455 & 0.00011128 & $4.8081 \mathrm{e}-05$ & $1.46508 \mathrm{e}+06$ & $2.9628 \mathrm{e}-148$ & Reject \\
\hline topic1456 & $9.3834 \mathrm{e}-05$ & $2.7988 \mathrm{e}-05$ & $1.26234 \mathrm{e}+06$ & $1.1564 \mathrm{e}-203$ & Reject \\
\hline topic1457 & 0.00010887 & $6.471 \mathrm{e}-05$ & $1.86102 \mathrm{e}+06$ & $2.4678 \mathrm{e}-65$ & Reject \\
\hline topic1458 & 0.00011784 & $8.2046 \mathrm{e}-05$ & $2.0265 \mathrm{e}+06$ & $3.045 \mathrm{e}-40$ & Reject \\
\hline topic1459 & 0.00014168 & 0.00011662 & $2.20865 \mathrm{e}+06$ & $3.1853 \mathrm{e}-21$ & Reject \\
\hline topic1460 & 7.6942e-05 & $4.1973 \mathrm{e}-05$ & $1.98708 \mathrm{e}+06$ & $1.0814 \mathrm{e}-46$ & Reject \\
\hline topic1461 & 0.00011178 & $7.2028 \mathrm{e}-05$ & $2.00202 \mathrm{e}+06$ & $2.855 \mathrm{e}-44$ & Reject \\
\hline topic1462 & 0.0001202 & $8.8713 \mathrm{e}-05$ & $1.97344 \mathrm{e}+06$ & $1.3083 \mathrm{e}-48$ & Reject \\
\hline topic1463 & 0.00012893 & 0.00014376 & $2.37418 \mathrm{e}+06$ & $6.2172 \mathrm{e}-09$ & Reject \\
\hline topic1464 & 0.00013026 & 0.00014624 & $2.48289 \mathrm{e}+06$ & 0.00058283 & Reject \\
\hline topic1465 & $7.5827 \mathrm{e}-05$ & $1.8762 \mathrm{e}-05$ & $1.26304 \mathrm{e}+06$ & 2.6143e-193 & Reject \\
\hline topic1466 & 0.00011972 & $8.5738 \mathrm{e}-05$ & $1.95961 \mathrm{e}+06$ & $1.871 \mathrm{e}-50$ & Reject \\
\hline topic1467 & 0.00012283 & 0.00011365 & $2.47364 \mathrm{e}+06$ & 0.00030674 & Reject \\
\hline topic1468 & $9.1305 \mathrm{e}-05$ & $6.8961 \mathrm{e}-05$ & $1.89543 \mathrm{e}+06$ & $1.6823 \mathrm{e}-60$ & Reject \\
\hline topic1469 & 0.00010801 & $7.5537 \mathrm{e}-05$ & $2.12797 \mathrm{e}+06$ & $5.2963 \mathrm{e}-29$ & Reject \\
\hline topic1470 & 0.00015263 & 0.00014021 & $2.54929 \mathrm{e}+06$ & 0.03884 & Reject \\
\hline topic1471 & 0.00010587 & $5.6203 \mathrm{e}-05$ & $1.61736 \mathrm{e}+06$ & 7.536e-113 & Reject \\
\hline topic1472 & 0.00010057 & $4.3877 \mathrm{e}-05$ & $1.58725 \mathrm{e}+06$ & $5.5672 \mathrm{e}-119$ & Reject \\
\hline topic1473 & 0.00011433 & $6.7318 \mathrm{e}-05$ & $1.85353 \mathrm{e}+06$ & $4.7228 \mathrm{e}-67$ & Reject \\
\hline topic1474 & 7.604e-08 & $1.5992 \mathrm{e}-07$ & 415900 & $3.8439 \mathrm{e}-19$ & Reject \\
\hline topic1475 & 0.00011213 & 0.00010192 & $2.56158 \mathrm{e}+06$ & 0.079061 & Accept \\
\hline topic1476 & $5.1467 \mathrm{e}-05$ & $1.3575 \mathrm{e}-05$ & $1.05983 \mathrm{e}+06$ & $5.1779 \mathrm{e}-267$ & Reject \\
\hline topic1477 & 0.00011668 & 0.0001249 & $2.47433 \mathrm{e}+06$ & 0.00026878 & Reject \\
\hline topic1478 & 0.00014917 & $8.0153 \mathrm{e}-05$ & $1.76026 \mathrm{e}+06$ & $1.8961 \mathrm{e}-83$ & Reject \\
\hline topic1479 & $8.5296 \mathrm{e}-05$ & $5.1287 \mathrm{e}-05$ & $2.08874 \mathrm{e}+06$ & $1.1804 \mathrm{e}-33$ & Reject \\
\hline topic1480 & $7.8047 \mathrm{e}-05$ & $7.372 \mathrm{e}-05$ & $2.4824 \mathrm{e}+06$ & 0.00051618 & Reject \\
\hline topic1481 & $8.2998 \mathrm{e}-05$ & $6.5311 \mathrm{e}-05$ & $2.00196 \mathrm{e}+06$ & $2.1779 \mathrm{e}-44$ & Reject \\
\hline topic1482 & $2.1032 \mathrm{e}-05$ & $9.8033 \mathrm{e}-06$ & $2.02062 \mathrm{e}+06$ & $1.2389 \mathrm{e}-41$ & Reject \\
\hline topic1483 & $9.9625 \mathrm{e}-05$ & $5.1402 \mathrm{e}-05$ & $1.80762 \mathrm{e}+06$ & $1.6656 \mathrm{e}-74$ & Reject \\
\hline topic1484 & $7.7398 \mathrm{e}-05$ & $6.6309 \mathrm{e}-05$ & $2.44034 \mathrm{e}+06$ & $1.7128 \mathrm{e}-05$ & Reject \\
\hline topic1485 & 0.00012987 & 0.00010896 & $2.13196 \mathrm{e}+06$ & $8.7611 \mathrm{e}-29$ & Reject \\
\hline topic1486 & 0.00013643 & 0.00010254 & $2.19743 \mathrm{e}+06$ & $5.4868 \mathrm{e}-22$ & Reject \\
\hline topic1487 & 0.00015936 & 0.00013408 & $2.32376 \mathrm{e}+06$ & $4.626 \mathrm{e}-12$ & Reject \\
\hline topic1488 & $1.2966 \mathrm{e}-05$ & $1.8869 \mathrm{e}-05$ & $2.16672 \mathrm{e}+06$ & $1.1431 \mathrm{e}-23$ & Reject \\
\hline topic1489 & $7.424 \mathrm{e}-05$ & $7.7702 \mathrm{e}-05$ & $2.56732 \mathrm{e}+06$ & 0.082497 & Accept \\
\hline topic1490 & 0.00013391 & 0.00011465 & $2.21085 \mathrm{e}+06$ & $1.2075 \mathrm{e}-20$ & Reject \\
\hline topic1491 & 0.00016441 & 0.00017461 & $2.52726 \mathrm{e}+06$ & 0.011259 & Reject \\
\hline topic1492 & 0.00010878 & $8.0675 \mathrm{e}-05$ & $2.35328 \mathrm{e}+06$ & $6.0013 \mathrm{e}-08$ & Reject \\
\hline topic1493 & 0.00014086 & 0.00012752 & $2.48476 \mathrm{e}+06$ & 0.00081129 & Reject \\
\hline topic1494 & $3.3447 \mathrm{e}-05$ & $1.9825 \mathrm{e}-05$ & $1.84812 \mathrm{e}+06$ & $4.0317 \mathrm{e}-68$ & Reject \\
\hline topic1495 & 0.00014434 & $7.7436 \mathrm{e}-05$ & $2.02029 \mathrm{e}+06$ & $3.3333 \mathrm{e}-40$ & Reject \\
\hline
\end{tabular}


Table A1 - Continued from previous page

\begin{tabular}{|c|c|c|c|c|c|}
\hline Feature & EN median & FL median & u-value & p-value & $\mathbf{H}_{0}$ \\
\hline topic1496 & 0.00014317 & $9.095 \mathrm{e}-05$ & $2.06172 \mathrm{e}+06$ & $2.5621 \mathrm{e}-36$ & Reject \\
\hline topic1497 & 0.00014235 & $6.5307 \mathrm{e}-05$ & $1.62401 \mathrm{e}+06$ & $1.0923 \mathrm{e}-110$ & Reject \\
\hline topic1498 & 0.0001607 & 0.00016428 & $2.62716 \mathrm{e}+06$ & 0.47819 & Accept \\
\hline topic1499 & 0.00015649 & 0.00010241 & $2.06601 \mathrm{e}+06$ & $2.8915 \mathrm{e}-36$ & Reject \\
\hline topic1500 & $9.1585 \mathrm{e}-05$ & $5.208 \mathrm{e}-05$ & $1.94738 \mathrm{e}+06$ & $5.709 \mathrm{e}-52$ & Reject \\
\hline topic1501 & 0.00011079 & $7.8952 \mathrm{e}-05$ & $2.04482 \mathrm{e}+06$ & $5.0099 \mathrm{e}-39$ & Reject \\
\hline topic1502 & $5.9245 \mathrm{e}-05$ & $3.5486 \mathrm{e}-05$ & $1.60928 \mathrm{e}+06$ & $1.267 \mathrm{e}-114$ & Reject \\
\hline topic1503 & 0.00013093 & 0.00010952 & $2.19741 \mathrm{e}+06$ & $2.8665 \mathrm{e}-22$ & Reject \\
\hline topic1504 & 0.00012825 & $5.5483 \mathrm{e}-05$ & $1.82683 \mathrm{e}+06$ & $1.5787 \mathrm{e}-70$ & Reject \\
\hline topic1505 & $9.4858 \mathrm{e}-05$ & $9.8052 \mathrm{e}-05$ & $2.60541 \mathrm{e}+06$ & 0.29482 & Accept \\
\hline topic1506 & 0.00010812 & 0.00013103 & $2.28821 \mathrm{e}+06$ & $1.9569 \mathrm{e}-14$ & Reject \\
\hline topic1507 & $6.3975 \mathrm{e}-06$ & $4.8886 \mathrm{e}-06$ & $2.34206 \mathrm{e}+06$ & $1.4937 \mathrm{e}-07$ & Reject \\
\hline topic1508 & $4.3345 \mathrm{e}-05$ & $3.8766 \mathrm{e}-05$ & $2.2422 \mathrm{e}+06$ & $3.5262 \mathrm{e}-18$ & Reject \\
\hline topic1509 & 0.00012722 & 0.0001228 & $2.53818 \mathrm{e}+06$ & 0.020778 & Reject \\
\hline topic1510 & 0.00012069 & 0.00017888 & $1.87096 \mathrm{e}+06$ & $1.8634 \mathrm{e}-64$ & Reject \\
\hline topic1511 & 0.00011318 & $8.9448 \mathrm{e}-05$ & $2.01156 \mathrm{e}+06$ & $1.7562 \mathrm{e}-43$ & Reject \\
\hline topic1512 & $6.7482 \mathrm{e}-05$ & $5.6068 \mathrm{e}-05$ & $2.29703 \mathrm{e}+06$ & $8.7165 \mathrm{e}-14$ & Reject \\
\hline topic1513 & $9.3703 \mathrm{e}-05$ & $8.7813 \mathrm{e}-05$ & $2.52268 \mathrm{e}+06$ & 0.0091418 & Reject \\
\hline topic1514 & 0.00015511 & 0.00014008 & $2.36308 \mathrm{e}+06$ & $1.8859 \mathrm{e}-09$ & Reject \\
\hline topic1515 & 0.00013955 & $7.2501 \mathrm{e}-05$ & $1.8439 \mathrm{e}+06$ & $9.6512 \mathrm{e}-68$ & Reject \\
\hline topic1516 & 0.00013484 & 0.00011715 & $2.24986 \mathrm{e}+06$ & $2.321 \mathrm{e}-17$ & Reject \\
\hline topic1517 & $9.1551 \mathrm{e}-05$ & $3.0493 \mathrm{e}-05$ & $1.244 \mathrm{e}+06$ & $1.2639 \mathrm{e}-207$ & Reject \\
\hline topic1518 & 0.0001481 & 0.00010911 & $1.96647 \mathrm{e}+06$ & $9.5601 \mathrm{e}-50$ & Reject \\
\hline topic1519 & 0.0001548 & 0.00017619 & $2.3832 \mathrm{e}+06$ & $2.2516 \mathrm{e}-08$ & Reject \\
\hline topic1520 & 0.00011802 & $8.2554 \mathrm{e}-05$ & $2.16034 \mathrm{e}+06$ & $1.0453 \mathrm{e}-25$ & Reject \\
\hline topic1521 & 0.00011103 & $9.161 \mathrm{e}-05$ & $2.29136 \mathrm{e}+06$ & $4.8381 \mathrm{e}-14$ & Reject \\
\hline topic1522 & $8.3971 \mathrm{e}-05$ & $4.4851 \mathrm{e}-05$ & $1.43861 \mathrm{e}+06$ & $2.0434 \mathrm{e}-155$ & Reject \\
\hline topic1523 & $3.8713 \mathrm{e}-05$ & $3.1677 \mathrm{e}-05$ & $2.12802 \mathrm{e}+06$ & $2.525 \mathrm{e}-29$ & Reject \\
\hline topic1524 & 0.00013429 & 0.00010791 & $2.16108 \mathrm{e}+06$ & $1.5864 \mathrm{e}-25$ & Reject \\
\hline topic1525 & $6.156 \mathrm{e}-05$ & $4.8097 \mathrm{e}-05$ & $2.22639 \mathrm{e}+06$ & $1.2566 \mathrm{e}-19$ & Reject \\
\hline topic1526 & 0.00012328 & 0.00014385 & $2.31256 \mathrm{e}+06$ & $9.4097 \mathrm{e}-13$ & Reject \\
\hline topic1527 & 0.0001178 & $9.3825 \mathrm{e}-05$ & $1.90889 \mathrm{e}+06$ & $3.1329 \mathrm{e}-58$ & Reject \\
\hline topic1528 & 0.00014204 & 0.00010852 & $2.20083 \mathrm{e}+06$ & $1.7853 \mathrm{e}-21$ & Reject \\
\hline topic1529 & $7.4478 \mathrm{e}-05$ & $9.374 \mathrm{e}-05$ & $2.2537 \mathrm{e}+06$ & $2.6582 \mathrm{e}-17$ & Reject \\
\hline topic1530 & $9.5656 \mathrm{e}-05$ & 0.00013247 & $2.3678 \mathrm{e}+06$ & $3.592 \mathrm{e}-09$ & Reject \\
\hline topic1531 & 0.00020563 & 0.00014063 & $2.16211 \mathrm{e}+06$ & $2.0615 \mathrm{e}-25$ & Reject \\
\hline topic1532 & 0.00012098 & $9.9286 \mathrm{e}-05$ & $2.1637 \mathrm{e}+06$ & $1.4435 \mathrm{e}-25$ & Reject \\
\hline topic1533 & $9.0778 \mathrm{e}-05$ & $7.1306 \mathrm{e}-05$ & $2.15723 \mathrm{e}+06$ & $5.0308 \mathrm{e}-26$ & Reject \\
\hline topic1534 & 0.00015017 & 0.00011235 & $2.08158 \mathrm{e}+06$ & $2.9265 \mathrm{e}-34$ & Reject \\
\hline topic1535 & 0.00011358 & $6.7135 \mathrm{e}-05$ & $1.62817 \mathrm{e}+06$ & $6.1316 \mathrm{e}-110$ & Reject \\
\hline topic1536 & 0.00018142 & 0.00011634 & $1.94234 \mathrm{e}+06$ & $1.0271 \mathrm{e}-52$ & Reject \\
\hline topic1537 & 0.00010603 & $7.7958 \mathrm{e}-05$ & $2.22043 \mathrm{e}+06$ & $4.5392 \mathrm{e}-20$ & Reject \\
\hline
\end{tabular}


Table A1 - Continued from previous page

\begin{tabular}{|c|c|c|c|c|c|}
\hline Feature & EN median & FL median & u-value & p-value & $\mathbf{H}_{\mathbf{0}}$ \\
\hline topic1538 & 0.0001064 & $2.2968 \mathrm{e}-05$ & $1.0762 \mathrm{e}+06$ & $4.7604 \mathrm{e}-259$ & Reject \\
\hline topic1539 & $5.239 \mathrm{e}-05$ & $1.8772 \mathrm{e}-05$ & $1.5261 \mathrm{e}+06$ & $1.5634 \mathrm{e}-133$ & Reject \\
\hline topic1540 & 0.0001263 & $7.7086 \mathrm{e}-05$ & $2.06359 \mathrm{e}+06$ & $1.1296 \mathrm{e}-36$ & Reject \\
\hline topic1541 & $7.0262 \mathrm{e}-05$ & $3.5712 \mathrm{e}-05$ & $1.83084 \mathrm{e}+06$ & $1.0452 \mathrm{e}-69$ & Reject \\
\hline topic1542 & $1.8719 \mathrm{e}-05$ & $1.8511 \mathrm{e}-05$ & $2.61609 \mathrm{e}+06$ & 0.39177 & Accept \\
\hline topic1543 & 0.0001445 & 0.00015134 & $2.55448 \mathrm{e}+06$ & 0.049623 & Reject \\
\hline topic1544 & 0.00012577 & 0.00010443 & $2.07301 \mathrm{e}+06$ & $1.1941 \mathrm{e}-35$ & Reject \\
\hline topic1545 & 0.00030248 & 0.0002288 & $1.96996 \mathrm{e}+06$ & $3.0278 \mathrm{e}-49$ & Reject \\
\hline topic1546 & 0.000137 & 0.00010959 & $2.12287 \mathrm{e}+06$ & 2.4671e-29 & Reject \\
\hline topic1547 & 0.00010958 & $8.848 \mathrm{e}-05$ & $2.21128 \mathrm{e}+06$ & $1.5815 \mathrm{e}-20$ & Reject \\
\hline topic1548 & 0.00015586 & 0.00010316 & $1.86758 \mathrm{e}+06$ & $9.7763 \mathrm{e}-63$ & Reject \\
\hline topic1549 & $5.4833 \mathrm{e}-05$ & $5.5506 \mathrm{e}-05$ & $2.62387 \mathrm{e}+06$ & 0.46934 & Accept \\
\hline topic1550 & $2.6688 \mathrm{e}-05$ & $1.9392 \mathrm{e}-06$ & 787854 & 0 & Reject \\
\hline topic1551 & 0.00011197 & 0.00010221 & $2.34421 \mathrm{e}+06$ & $9.968 \mathrm{e}-11$ & Reject \\
\hline topic1552 & 0.00010051 & 0.00011623 & $2.24412 \mathrm{e}+06$ & $5.2111 \mathrm{e}-18$ & Reject \\
\hline topic1553 & 0.00014882 & 0.00010369 & $2.24839 \mathrm{e}+06$ & $2.1501 \mathrm{e}-17$ & Reject \\
\hline topic1554 & $9.791 \mathrm{e}-05$ & $9.6522 \mathrm{e}-05$ & $2.54221 \mathrm{e}+06$ & 0.028824 & Reject \\
\hline topic1555 & $5.8409 \mathrm{e}-05$ & $4.1988 \mathrm{e}-05$ & $2.23523 \mathrm{e}+06$ & $1.7341 \mathrm{e}-16$ & Reject \\
\hline topic1556 & 0.00016296 & 0.00019777 & $2.22446 \mathrm{e}+06$ & $8.5005 \mathrm{e}-20$ & Reject \\
\hline topic1557 & 0.00010239 & $9.4354 \mathrm{e}-05$ & $2.48762 \mathrm{e}+06$ & 0.00084662 & Reject \\
\hline topic1558 & $5.3428 \mathrm{e}-05$ & $3.9661 \mathrm{e}-05$ & $2.09567 \mathrm{e}+06$ & $1.6687 \mathrm{e}-32$ & Reject \\
\hline topic1559 & 0.0001334 & 0.00010381 & $2.25559 \mathrm{e}+06$ & $5.6896 \mathrm{e}-17$ & Reject \\
\hline topic1560 & $9.4558 \mathrm{e}-05$ & $7.4485 \mathrm{e}-05$ & $2.12849 \mathrm{e}+06$ & $4.6568 \mathrm{e}-29$ & Reject \\
\hline topic1561 & 0.00013514 & $9.5832 \mathrm{e}-05$ & $1.88401 \mathrm{e}+06$ & $6.9209 \mathrm{e}-62$ & Reject \\
\hline topic1562 & $7.4804 \mathrm{e}-05$ & $3.8731 \mathrm{e}-05$ & $1.889 \mathrm{e}+06$ & $1.2478 \mathrm{e}-60$ & Reject \\
\hline topic1563 & $9.0007 \mathrm{e}-05$ & $6.9871 \mathrm{e}-05$ & $2.27113 \mathrm{e}+06$ & $8.1337 \mathrm{e}-16$ & Reject \\
\hline topic1564 & 0.00012325 & 0.00011608 & $2.51641 \mathrm{e}+06$ & 0.0066849 & Reject \\
\hline topic1565 & $4.2047 \mathrm{e}-05$ & $1.4773 \mathrm{e}-05$ & $1.5969 \mathrm{e}+06$ & $3.3206 \mathrm{e}-114$ & Reject \\
\hline topic1566 & 0.00014918 & 0.00014789 & $2.61056 \mathrm{e}+06$ & 0.35433 & Accept \\
\hline topic1567 & 0.0001343 & 0.00012041 & $2.45179 \mathrm{e}+06$ & $4.5174 \mathrm{e}-05$ & Reject \\
\hline topic1568 & $9.3584 \mathrm{e}-05$ & $5.7591 \mathrm{e}-05$ & $2.06462 \mathrm{e}+06$ & $2.0087 \mathrm{e}-36$ & Reject \\
\hline topic1569 & $4.5616 \mathrm{e}-05$ & $6.1393 \mathrm{e}-05$ & $2.27260 \mathrm{e}+06$ & $1.0615 \mathrm{e}-15$ & Reject \\
\hline topic1570 & $8.4864 \mathrm{e}-05$ & $4.9004 \mathrm{e}-05$ & $1.88434 \mathrm{e}+06$ & $5.695 \mathrm{e}-62$ & Reject \\
\hline topic1571 & 0.00012224 & $4.5543 \mathrm{e}-05$ & $1.30944 \mathrm{e}+06$ & $1.4379 \mathrm{e}-189$ & Reject \\
\hline topic1572 & $9.1884 \mathrm{e}-05$ & $6.4234 \mathrm{e}-05$ & $2.26659 \mathrm{e}+06$ & $4.3453 \mathrm{e}-16$ & Reject \\
\hline topic1573 & $5.9869 \mathrm{e}-05$ & $3.3809 \mathrm{e}-05$ & $1.99486 \mathrm{e}+06$ & $1.4602 \mathrm{e}-44$ & Reject \\
\hline topic1574 & $4.2821 \mathrm{e}-05$ & $1.3695 \mathrm{e}-05$ & $1.41023 \mathrm{e}+06$ & $3.3426 \mathrm{e}-162$ & Reject \\
\hline topic1575 & $4.6254 \mathrm{e}-05$ & $1.7834 \mathrm{e}-05$ & $1.57666 \mathrm{e}+06$ & $1.8768 \mathrm{e}-120$ & Reject \\
\hline topic1576 & 0.00012545 & $5.7304 \mathrm{e}-05$ & $1.26014 \mathrm{e}+06$ & $1.1069 \mathrm{e}-203$ & Reject \\
\hline topic1577 & 0.00011104 & 0.00011417 & $2.62053 \mathrm{e}+06$ & 0.45916 & Accept \\
\hline topic1578 & 0.00012967 & $6.1573 \mathrm{e}-05$ & $1.43322 \mathrm{e}+06$ & $1.4047 \mathrm{e}-156$ & Reject \\
\hline topic1579 & 0.0001443 & 0.00010924 & $2.0251 \mathrm{e}+06$ & $1.4796 \mathrm{e}-41$ & Reject \\
\hline
\end{tabular}


Table A1 - Continued from previous page

\begin{tabular}{|c|c|c|c|c|c|}
\hline Feature & EN median & FL median & u-value & p-value & $\mathbf{H}_{0}$ \\
\hline topic1580 & 0.0001257 & 0.0001104 & $2.46698 \mathrm{e}+06$ & 0.00015929 & Reject \\
\hline topic1581 & $9.8405 \mathrm{e}-05$ & $9.258 \mathrm{e}-05$ & $2.48097 \mathrm{e}+06$ & 0.00054868 & Reject \\
\hline topic1582 & $9.8852 \mathrm{e}-05$ & $8.7522 \mathrm{e}-05$ & $2.45192 \mathrm{e}+06$ & $5.0335 \mathrm{e}-05$ & Reject \\
\hline topic1583 & 0.00010152 & $5.1789 \mathrm{e}-05$ & $2.12537 \mathrm{e}+06$ & $1.683 \mathrm{e}-29$ & Reject \\
\hline topic1584 & $9.4452 \mathrm{e}-05$ & $5.9771 \mathrm{e}-05$ & $1.9778 \mathrm{e}+06$ & $7.4572 \mathrm{e}-48$ & Reject \\
\hline topic1585 & $3.2984 \mathrm{e}-08$ & $4.5945 \mathrm{e}-08$ & 811416 & $2.9102 \mathrm{e}-05$ & Reject \\
\hline topic1586 & $6.0108 \mathrm{e}-05$ & $4.5929 \mathrm{e}-05$ & $2.23921 \mathrm{e}+06$ & $1.6243 \mathrm{e}-18$ & Reject \\
\hline topic1587 & $9.2888 \mathrm{e}-05$ & $8.2733 \mathrm{e}-05$ & $2.39968 \mathrm{e}+06$ & $1.6765 \mathrm{e}-07$ & Reject \\
\hline topic1588 & $7.3348 \mathrm{e}-05$ & $6.4015 \mathrm{e}-05$ & $2.44763 \mathrm{e}+06$ & $2.7495 \mathrm{e}-05$ & Reject \\
\hline topic1589 & $6.6051 \mathrm{e}-05$ & 7.3194e-05 & $2.51095 \mathrm{e}+06$ & 0.0047517 & Reject \\
\hline topic1590 & 0.00013968 & $9.7113 \mathrm{e}-05$ & $2.05372 \mathrm{e}+06$ & $1.6079 \mathrm{e}-37$ & Reject \\
\hline topic1591 & $9.0924 \mathrm{e}-05$ & $6.9208 \mathrm{e}-05$ & $2.22112 \mathrm{e}+06$ & $1.001 \mathrm{e}-19$ & Reject \\
\hline topic1592 & $1.2928 \mathrm{e}-05$ & $1.1812 \mathrm{e}-05$ & $2.44746 \mathrm{e}+06$ & $2.7316 \mathrm{e}-05$ & Reject \\
\hline topic1593 & $5.3725 \mathrm{e}-08$ & $7.518 \mathrm{e}-08$ & $1.57138 \mathrm{e}+06$ & $6.0738 \mathrm{e}-11$ & Reject \\
\hline topic1594 & 0.00012291 & $9.9569 \mathrm{e}-05$ & $2.23648 \mathrm{e}+06$ & $1.1717 \mathrm{e}-18$ & Reject \\
\hline topic1595 & 0.00014831 & $7.7502 \mathrm{e}-05$ & $1.40121 \mathrm{e}+06$ & $9.367 \mathrm{e}-164$ & Reject \\
\hline topic1596 & $6.4097 \mathrm{e}-05$ & $6.4241 \mathrm{e}-05$ & $2.627 \mathrm{e}+06$ & 0.47679 & Accept \\
\hline topic1597 & 0.00013912 & $5.2288 \mathrm{e}-05$ & $1.44439 \mathrm{e}+06$ & $6.5341 \mathrm{e}-154$ & Reject \\
\hline topic1598 & 0.00011102 & $6.7778 \mathrm{e}-05$ & $1.97606 \mathrm{e}+06$ & $4.2247 \mathrm{e}-48$ & Reject \\
\hline topic1599 & 0.00015576 & 0.00015836 & $2.57465 \mathrm{e}+06$ & 0.13093 & Accept \\
\hline topic1600 & 0.00014609 & 0.00011908 & $2.11373 \mathrm{e}+06$ & $1.1247 \mathrm{e}-30$ & Reject \\
\hline topic1601 & $4.8858 \mathrm{e}-05$ & $4.0751 \mathrm{e}-05$ & $2.26283 \mathrm{e}+06$ & $2.1447 \mathrm{e}-16$ & Reject \\
\hline topic1602 & 0.00013364 & 0.00010356 & $1.94637 \mathrm{e}+06$ & $1.5532 \mathrm{e}-52$ & Reject \\
\hline topic1603 & $1.75 \mathrm{e}-08$ & $8.2163 \mathrm{e}-08$ & 412118 & $9.9677 \mathrm{e}-67$ & Reject \\
\hline topic1604 & $6.7622 \mathrm{e}-05$ & $6.6638 \mathrm{e}-05$ & $2.62032 \mathrm{e}+06$ & 0.44944 & Accept \\
\hline topic1605 & 0.00011402 & $7.6446 \mathrm{e}-05$ & $2.2057 \mathrm{e}+06$ & $4.1702 \mathrm{e}-21$ & Reject \\
\hline topic1606 & $1.7846 \mathrm{e}-05$ & $3.8171 \mathrm{e}-06$ & $1.582 \mathrm{e}+06$ & $8.1879 \mathrm{e}-118$ & Reject \\
\hline topic1607 & $9.3937 \mathrm{e}-05$ & $8.6961 \mathrm{e}-05$ & $2.48733 \mathrm{e}+06$ & 0.0020856 & Reject \\
\hline topic1608 & $8.1419 \mathrm{e}-05$ & $6.0972 \mathrm{e}-05$ & $2.37945 \mathrm{e}+06$ & $9.4962 \mathrm{e}-08$ & Reject \\
\hline topic1609 & $7.3969 \mathrm{e}-05$ & $3.8448 \mathrm{e}-05$ & $1.39429 \mathrm{e}+06$ & $2.2034 \mathrm{e}-166$ & Reject \\
\hline topic1610 & $8.6092 \mathrm{e}-05$ & $4.9818 \mathrm{e}-05$ & $1.49687 \mathrm{e}+06$ & $8.9939 \mathrm{e}-141$ & Reject \\
\hline topic1611 & $8.2609 \mathrm{e}-05$ & $5.9872 \mathrm{e}-05$ & $1.99169 \mathrm{e}+06$ & $6.4533 \mathrm{e}-46$ & Reject \\
\hline topic1612 & 0.00012893 & $8.0978 \mathrm{e}-05$ & $1.83664 \mathrm{e}+06$ & $6.68 \mathrm{e}-70$ & Reject \\
\hline topic1613 & 0.00012552 & $7.7908 \mathrm{e}-05$ & $1.86611 \mathrm{e}+06$ & $4.235 \mathrm{e}-65$ & Reject \\
\hline topic1614 & 0.0001606 & 0.0001365 & $2.24661 \mathrm{e}+06$ & $8.4264 \mathrm{e}-18$ & Reject \\
\hline topic1615 & 0.0001079 & $8.3341 \mathrm{e}-05$ & $2.25623 \mathrm{e}+06$ & $1.6511 \mathrm{e}-16$ & Reject \\
\hline topic1616 & 0.00011545 & 0.00010195 & $2.30625 \mathrm{e}+06$ & $3.3944 \mathrm{e}-13$ & Reject \\
\hline topic1617 & $6.1202 \mathrm{e}-05$ & $7.3844 \mathrm{e}-05$ & $2.48726 \mathrm{e}+06$ & 0.0010608 & Reject \\
\hline topic1618 & $8.1293 \mathrm{e}-05$ & $3.78 \mathrm{e}-05$ & $1.67552 \mathrm{e}+06$ & $3.8071 \mathrm{e}-100$ & Reject \\
\hline topic1619 & $8.3849 \mathrm{e}-05$ & $2.6753 \mathrm{e}-05$ & $2.03568 \mathrm{e}+06$ & $4.5834 \mathrm{e}-40$ & Reject \\
\hline topic1620 & $1.9975 \mathrm{e}-05$ & $6.7095 \mathrm{e}-06$ & $1.67772 \mathrm{e}+06$ & $6.8444 \mathrm{e}-100$ & Reject \\
\hline topic1621 & $4.9307 \mathrm{e}-05$ & $3.9366 \mathrm{e}-05$ & $2.27708 \mathrm{e}+06$ & $2.8562 \mathrm{e}-15$ & Reject \\
\hline
\end{tabular}


Table A1 - Continued from previous page

\begin{tabular}{|c|c|c|c|c|c|}
\hline Feature & EN median & FL median & u-value & p-value & $\mathbf{H}_{0}$ \\
\hline topic1622 & $5.4085 \mathrm{e}-05$ & $4.2926 \mathrm{e}-05$ & $2.18995 \mathrm{e}+06$ & $7.0683 \mathrm{e}-23$ & Reject \\
\hline topic1623 & 0.00012542 & 0.00010982 & $2.43507 \mathrm{e}+06$ & $1.9905 \mathrm{e}-05$ & Reject \\
\hline topic1624 & $3.3011 \mathrm{e}-05$ & $1.4745 \mathrm{e}-05$ & $1.9868 \mathrm{e}+06$ & $4.3692 \mathrm{e}-46$ & Reject \\
\hline topic1625 & 0.00012192 & $9.8909 \mathrm{e}-05$ & $2.35702 \mathrm{e}+06$ & $8.3515 \mathrm{e}-10$ & Reject \\
\hline topic1626 & $4.5609 \mathrm{e}-05$ & $2.3709 \mathrm{e}-05$ & $1.44869 \mathrm{e}+06$ & $1.3135 \mathrm{e}-152$ & Reject \\
\hline topic1627 & $8.1858 \mathrm{e}-05$ & $6.8466 \mathrm{e}-05$ & $2.50618 \mathrm{e}+06$ & 0.0037225 & Reject \\
\hline topic1628 & 0.00012509 & 0.00011167 & $2.5121 \mathrm{e}+06$ & 0.006307 & Reject \\
\hline topic1629 & 0.00013353 & $7.8187 \mathrm{e}-05$ & $1.96227 \mathrm{e}+06$ & $3.7631 \mathrm{e}-46$ & Reject \\
\hline topic1630 & $7.5258 \mathrm{e}-05$ & $9.0145 \mathrm{e}-05$ & $2.35847 \mathrm{e}+06$ & $8.7595 \mathrm{e}-10$ & Reject \\
\hline topic1631 & 0.00017398 & 0.00015023 & $2.31066 \mathrm{e}+06$ & $5.8169 \mathrm{e}-13$ & Reject \\
\hline topic1632 & $9.5288 \mathrm{e}-05$ & $8.2707 \mathrm{e}-05$ & $2.43608 \mathrm{e}+06$ & $1.3965 \mathrm{e}-05$ & Reject \\
\hline topic1633 & 0.00012606 & 0.00011647 & $2.46553 \mathrm{e}+06$ & 0.00018713 & Reject \\
\hline topic1634 & 0.00014375 & 0.00010858 & $2.02021 \mathrm{e}+06$ & $3.2785 \mathrm{e}-42$ & Reject \\
\hline topic1635 & 0.00016331 & 0.00013523 & $2.27259 \mathrm{e}+06$ & $1.0402 \mathrm{e}-15$ & Reject \\
\hline topic1636 & 0.00014037 & $7.8554 \mathrm{e}-05$ & $1.88369 \mathrm{e}+06$ & $5.0921 \mathrm{e}-60$ & Reject \\
\hline topic1637 & 0.0001286 & $9.3103 \mathrm{e}-05$ & $2.08386 \mathrm{e}+06$ & $4.1337 \mathrm{e}-34$ & Reject \\
\hline topic1638 & $1.822 \mathrm{e}-05$ & $1.0572 \mathrm{e}-05$ & $1.82524 \mathrm{e}+06$ & $2.9716 \mathrm{e}-71$ & Reject \\
\hline topic1639 & 0.00010148 & $3.339 \mathrm{e}-05$ & $1.56386 \mathrm{e}+06$ & $1.5764 \mathrm{e}-123$ & Reject \\
\hline topic1640 & 0.00013446 & 0.00010362 & $2.01741 \mathrm{e}+06$ & $1.3966 \mathrm{e}-42$ & Reject \\
\hline topic1641 & $7.5341 \mathrm{e}-05$ & $4.9627 \mathrm{e}-05$ & $1.60865 \mathrm{e}+06$ & $9.2103 \mathrm{e}-115$ & Reject \\
\hline topic1642 & 0.00010199 & $1.4332 \mathrm{e}-05$ & $1.15717 \mathrm{e}+06$ & $3.9847 \mathrm{e}-236$ & Reject \\
\hline topic1643 & $9.6072 \mathrm{e}-05$ & $9.4218 \mathrm{e}-05$ & $2.59606 \mathrm{e}+06$ & 0.27722 & Accept \\
\hline topic1644 & 0.00012667 & 0.00010959 & $2.23938 \mathrm{e}+06$ & $3.0854 \mathrm{e}-18$ & Reject \\
\hline topic1645 & $9.153 \mathrm{e}-05$ & $9.7725 \mathrm{e}-05$ & $2.51530 \mathrm{e}+06$ & 0.0061988 & Reject \\
\hline topic1646 & $4.9595 \mathrm{e}-05$ & $3.9726 \mathrm{e}-05$ & $2.19493 \mathrm{e}+06$ & $2.102 \mathrm{e}-22$ & Reject \\
\hline topic1647 & $3.6627 \mathrm{e}-05$ & $3.5773 \mathrm{e}-05$ & $2.53381 \mathrm{e}+06$ & 0.018609 & Reject \\
\hline topic1648 & 0.00014703 & 0.00010963 & $2.08749 \mathrm{e}+06$ & $8.4154 \mathrm{e}-34$ & Reject \\
\hline topic1649 & 0.00014521 & 0.00012441 & $2.31221 \mathrm{e}+06$ & $7.4695 \mathrm{e}-13$ & Reject \\
\hline topic1650 & 0.00017819 & 0.00015752 & $2.32361 \mathrm{e}+06$ & $4.5196 \mathrm{e}-12$ & Reject \\
\hline topic1651 & 0.00012846 & $9.6392 \mathrm{e}-05$ & $2.04456 \mathrm{e}+06$ & $8.1224 \mathrm{e}-39$ & Reject \\
\hline topic1652 & 0.00012595 & $6.0088 \mathrm{e}-05$ & $1.63895 \mathrm{e}+06$ & $4.3749 \mathrm{e}-107$ & Reject \\
\hline topic1653 & 0.00011747 & $8.7545 \mathrm{e}-05$ & $2.02478 \mathrm{e}+06$ & $1.8151 \mathrm{e}-41$ & Reject \\
\hline topic1654 & 0.00013533 & 0.00014248 & $2.54349 \mathrm{e}+06$ & 0.029142 & Reject \\
\hline topic1655 & 0.0001448 & $9.148 \mathrm{e}-05$ & $1.8265 \mathrm{e}+06$ & $2.5042 \mathrm{e}-71$ & Reject \\
\hline topic1656 & $5.4414 \mathrm{e}-05$ & $3.5182 \mathrm{e}-05$ & $2.02859 \mathrm{e}+06$ & $4.1152 \mathrm{e}-41$ & Reject \\
\hline topic1657 & 0.00019173 & 0.00013705 & $2.08103 \mathrm{e}+06$ & 2.3996e-34 & Reject \\
\hline topic1658 & 0.00016476 & 0.00013758 & $2.30473 \mathrm{e}+06$ & 2.6442e-13 & Reject \\
\hline topic1659 & 0.00013977 & $8.4299 \mathrm{e}-05$ & $1.8029 \mathrm{e}+06$ & $2.5929 \mathrm{e}-75$ & Reject \\
\hline topic1660 & 0.00010048 & $2.048 \mathrm{e}-05$ & $1.66274 \mathrm{e}+06$ & $6.6422 \mathrm{e}-102$ & Reject \\
\hline topic1661 & 0.00015419 & 0.00017933 & $2.27831 \mathrm{e}+06$ & $2.4246 \mathrm{e}-15$ & Reject \\
\hline topic1662 & 0.00020041 & 0.00013711 & $1.62119 \mathrm{e}+06$ & $4.9499 \mathrm{e}-112$ & Reject \\
\hline topic1663 & $4.8754 \mathrm{e}-05$ & $2.3521 \mathrm{e}-05$ & $2.02034 \mathrm{e}+06$ & $2.0515 \mathrm{e}-39$ & Reject \\
\hline
\end{tabular}


Table A1 - Continued from previous page

\begin{tabular}{|c|c|c|c|c|c|}
\hline Feature & EN median & FL median & u-value & p-value & $\mathbf{H}_{0}$ \\
\hline topic1664 & $4.6694 \mathrm{e}-05$ & $4.734 \mathrm{e}-05$ & $2.60898 \mathrm{e}+06$ & 0.32282 & Accept \\
\hline topic1665 & $2.0179 \mathrm{e}-05$ & $7.0294 \mathrm{e}-05$ & $1.61634 \mathrm{e}+06$ & $3.8474 \mathrm{e}-109$ & Reject \\
\hline topic1666 & 0.00016246 & 0.00012474 & $2.24972 \mathrm{e}+06$ & $4.9894 \mathrm{e}-17$ & Reject \\
\hline topic1667 & 4613 & 736 & $2.16455 \mathrm{e}+06$ & $e-24$ & Reject \\
\hline topic1668 & $9.7692 \mathrm{e}-05$ & 7.6293e-05 & $2.17342 \mathrm{e}+06$ & $989 \mathrm{e}-24$ & Reject \\
\hline topic1669 & 0.00010401 & $6.4071 \mathrm{e}-05$ & $1.83838 \mathrm{e}+06$ & $5.1955 \mathrm{e}-69$ & Reject \\
\hline topic1670 & $7.3169 \mathrm{e}-05$ & $7.0777 \mathrm{e}-05$ & $2.6055 \mathrm{e}+06$ & 0.29552 & Accept \\
\hline topic1671 & 0.00010481 & $7.6658 \mathrm{e}-05$ & $1.87434 \mathrm{e}+06$ & 6.691e-64 & Reject \\
\hline topic1672 & e-05 & $2.8879 \mathrm{e}-05$ & $2.2951 \mathrm{e}+06$ & $1.3036 \mathrm{e}-13$ & Reject \\
\hline topic1673 & 0.00013944 & $8.5886 \mathrm{e}-05$ & $2.01738 \mathrm{e}+06$ & $1.1137 \mathrm{e}-41$ & Reject \\
\hline topic1674 & $9.8032 \mathrm{e}-05$ & $1 \mathrm{e}-05$ & $2.41812 \mathrm{e}+06$ & $1.2128 \mathrm{e}-06$ & Reject \\
\hline topi & 0.00014142 & 0.00014205 & $2.61458 \mathrm{e}+06$ & 0.36879 & Accept \\
\hline topic1676 & $2.6222 \mathrm{e}-05$ & $2.5552 \mathrm{e}-05$ & $2.60623 \mathrm{e}+06$ & 0.34643 & Accept \\
\hline topic1677 & e-05 & 0.00 & $2.18565 \mathrm{e}+06$ & $5.4835 \mathrm{e}-23$ & Reject \\
\hline & 5 & $2.8524 \mathrm{e}-05$ & & -29 & \\
\hline topic1679 & $2.2067 \mathrm{e}-05$ & $7.518 \mathrm{e}-06$ & $1.27557 \mathrm{e}+06$ & $4.8936 \mathrm{e}-200$ & Reject \\
\hline top & $9.8699 \mathrm{e}-05$ & $7.2115 \mathrm{e}-05$ & $2.08454 \mathrm{e}+06$ & e-34 & Reject \\
\hline topic1681 & 0.00012211 & $9.8309 \mathrm{e}-05$ & $2.08656 \mathrm{e}+06$ & $4.9637 \mathrm{e}-34$ & Reject \\
\hline 1682 & $2-05$ & $7.3011 \mathrm{e}-05$ & $\mathrm{e}+06$ & e-20 & Reject \\
\hline & & & & & \\
\hline topic1684 & 0.00014208 & $8.09 \mathrm{e}-05$ & $1.72343 \mathrm{e}+06$ & $1.5931 \mathrm{e}-90$ & Reject \\
\hline 1685 & 0.00010201 & 00011999 & $2.2288 \mathrm{e}+06$ & e-19 & Reject \\
\hline 1686 & 0.00013633 & 0.00017944 & $2.26014 \mathrm{e}+06$ & 1.327 & Reject \\
\hline 1687 & e-05 & $2.3821 \mathrm{e}-05$ & $1 e+06$ & $e-23$ & Reject \\
\hline topic1688 & e-05 & $2.8434 \mathrm{e}-05$ & $8 \mathrm{e}+06$ & 2.002 & Reject \\
\hline top & 0.000 & $9.9549 \mathrm{e}-05$ & $1.96605 \mathrm{e}+06$ & $1.1502 \mathrm{e}-49$ & Reject \\
\hline 690 & e-05 & $2 e-05$ & $2.53041 \mathrm{e}+06$ & 006 & Reject \\
\hline & 0.00016421 & 0.00012243 & $1.996 \mathrm{e}+06$ & 2.523 & Reject \\
\hline & 7439e-06 & $388 \mathrm{e}-05$ & $5 e+06$ & $3.1375 \mathrm{e}-43$ & Reject \\
\hline & 2971 & 013377 & -06 & 122 & Accept \\
\hline 1694 & $2.2055 \mathrm{e}-05$ & $1.7869 \mathrm{e}-05$ & $2.37748 \mathrm{e}+06$ & $1.4452 \mathrm{e}-08$ & Reject \\
\hline topic1695 & $1951 \mathrm{e}-07$ & $1.3419 \mathrm{e}-07$ & $2.24572 \mathrm{e}+06$ & 0.38819 & Accept \\
\hline & 0495 & $7.1857 \mathrm{e}-05$ & $2.21433 \mathrm{e}+06$ & $1.3118 \mathrm{e}-20$ & Reject \\
\hline & e-05 & $2.6013 \mathrm{e}-05$ & $1.566 \mathrm{e}+06$ & $5.0767 \mathrm{e}-124$ & Reject \\
\hline 1698 & 0.00014338 & $9.5407 \mathrm{e}-05$ & $2.10905 \mathrm{e}+06$ & $4.2745 \mathrm{e}-31$ & Reject \\
\hline & 0.00 & 0.00016868 & $2.1811 \mathrm{e}+06$ & $1.2227 \mathrm{e}-23$ & Reject \\
\hline topic1700 & 0.00015718 & $7.2547 \mathrm{e}-05$ & $1.23566 \mathrm{e}+06$ & $3.2042 \mathrm{e}-211$ & Reject \\
\hline & 0.00011245 & $9.6063 \mathrm{e}-05$ & $2.29075 \mathrm{e}+06$ & $2.1199 \mathrm{e}-14$ & Reject \\
\hline & 0.00017027 & 0.00011345 & $1.67576 \mathrm{e}+06$ & $1.2708 \mathrm{e}-100$ & Reject \\
\hline topic1703 & $9.1337 \mathrm{e}-05$ & $5.8552 \mathrm{e}-05$ & $1.77037 \mathrm{e}+06$ & $3.0579 \mathrm{e}-81$ & Reject \\
\hline & $5.3937 \mathrm{e}-05$ & $3.6372 \mathrm{e}-05$ & $2.31926 \mathrm{e}+06$ & $1.2127 \mathrm{e}-11$ & Reject \\
\hline topic1705 & 0.00011227 & $7.6869 \mathrm{e}-05$ & $2.08811 \mathrm{e}+06$ & $7.556 \mathrm{e}-34$ & Reject \\
\hline
\end{tabular}


Table A1 - Continued from previous page

\begin{tabular}{|c|c|c|c|c|c|}
\hline Feature & EN median & FL median & u-value & p-value & $\mathbf{H}_{0}$ \\
\hline topic1706 & $2.6229 \mathrm{e}-05$ & $1.0992 \mathrm{e}-05$ & $2.03703 \mathrm{e}+06$ & $3.9859 \mathrm{e}-39$ & Reject \\
\hline topic1707 & 0.00011993 & 0.00013005 & $2.52125 \mathrm{e}+06$ & 0.010154 & Reject \\
\hline topic1708 & 0.00013763 & $7.4999 \mathrm{e}-05$ & $1.51161 \mathrm{e}+06$ & $8.2419 \mathrm{e}-137$ & Reject \\
\hline topic1709 & $2.2499 \mathrm{e}-05$ & $3.942 \mathrm{e}-05$ & $1.93747 \mathrm{e}+06$ & $9.2908 \mathrm{e}-43$ & Reject \\
\hline topic1710 & 0.00018885 & 0.00014657 & $2.08961 \mathrm{e}+06$ & $1.135 \mathrm{e}-33$ & Reject \\
\hline topic1711 & 0.00016757 & 0.00016346 & $2.59312 \mathrm{e}+06$ & 0.22346 & Accept \\
\hline topic1712 & 0.00025307 & 0.00017196 & $1.96696 \mathrm{e}+06$ & $8.6169 \mathrm{e}-48$ & Reject \\
\hline topic1713 & $1.8266 \mathrm{e}-05$ & $8.6814 \mathrm{e}-06$ & $1.77586 \mathrm{e}+06$ & $4.7261 \mathrm{e}-81$ & Reject \\
\hline topic1714 & $5.3242 \mathrm{e}-05$ & $4.4012 \mathrm{e}-05$ & $2.22083 \mathrm{e}+06$ & $6.2424 \mathrm{e}-20$ & Reject \\
\hline topic1715 & 0.00014278 & 0.00015533 & $2.51046 \mathrm{e}+06$ & 0.0039552 & Reject \\
\hline topic1716 & 0.00013141 & $9.7551 \mathrm{e}-05$ & $2.1604 \mathrm{e}+06$ & $1.085 \mathrm{e}-25$ & Reject \\
\hline topic1717 & 0.00013604 & $7.1925 \mathrm{e}-05$ & $1.4654 \mathrm{e}+06$ & $1.2353 \mathrm{e}-146$ & Reject \\
\hline topic1718 & 0.00013112 & 0.00010754 & $2.36977 \mathrm{e}+06$ & 4.6617e-09 & Reject \\
\hline topic1719 & 0.00016473 & 0.00012422 & $2.06275 \mathrm{e}+06$ & $1.1838 \mathrm{e}-36$ & Reject \\
\hline topic1720 & $8.4774 \mathrm{e}-05$ & $5.2816 \mathrm{e}-05$ & $2.08575 \mathrm{e}+06$ & $5.2496 \mathrm{e}-34$ & Reject \\
\hline topic1721 & 0.00013832 & 0.00012138 & $2.36069 \mathrm{e}+06$ & $1.1872 \mathrm{e}-09$ & Reject \\
\hline topic1722 & $6.8308 \mathrm{e}-05$ & $3.4642 \mathrm{e}-05$ & $1.74908 \mathrm{e}+06$ & $9.0728 \mathrm{e}-85$ & Reject \\
\hline topic1723 & $4.8329 \mathrm{e}-05$ & $4.2155 \mathrm{e}-05$ & $2.47246 \mathrm{e}+06$ & 0.00027373 & Reject \\
\hline topic1724 & $4.0737 \mathrm{e}-05$ & $2.4271 \mathrm{e}-05$ & $1.55808 \mathrm{e}+06$ & $5.306 \mathrm{e}-126$ & Reject \\
\hline topic1725 & 0.00019196 & 0.00014897 & $2.08655 \mathrm{e}+06$ & $6.5211 \mathrm{e}-34$ & Reject \\
\hline topic1726 & $5.452 \mathrm{e}-05$ & $4.4376 \mathrm{e}-05$ & $2.28747 \mathrm{e}+06$ & $7.408 \mathrm{e}-14$ & Reject \\
\hline topic1727 & 0.00016121 & 0.00014465 & $2.36630 \mathrm{e}+06$ & $2.5393 \mathrm{e}-09$ & Reject \\
\hline topic1728 & $5.4573 \mathrm{e}-05$ & $4.216 \mathrm{e}-05$ & $2.23525 \mathrm{e}+06$ & $7.4374 \mathrm{e}-19$ & Reject \\
\hline topic1729 & $8.2877 \mathrm{e}-05$ & $6.2701 \mathrm{e}-05$ & $2.11934 \mathrm{e}+06$ & $2.8014 \mathrm{e}-30$ & Reject \\
\hline topic1730 & 0.00012051 & 0.00016915 & $2.09136 \mathrm{e}+06$ & $2.3897 \mathrm{e}-33$ & Reject \\
\hline topic1731 & $8.6695 \mathrm{e}-05$ & $4.9117 \mathrm{e}-05$ & $1.91974 \mathrm{e}+06$ & $2.248 \mathrm{e}-55$ & Reject \\
\hline topic1732 & $8.3616 \mathrm{e}-05$ & $4.7127 \mathrm{e}-05$ & $1.59020 \mathrm{e}+06$ & 7.1972e-119 & Reject \\
\hline topic1733 & 0.00013315 & 0.00010853 & $2.1899 \mathrm{e}+06$ & $8.8128 \mathrm{e}-23$ & Reject \\
\hline topic1734 & 0.00014323 & 0.00011603 & $2.22421 \mathrm{e}+06$ & $9.8243 \mathrm{e}-20$ & Reject \\
\hline topic1735 & $3.7876 \mathrm{e}-05$ & $2.4373 \mathrm{e}-05$ & $2.21886 \mathrm{e}+06$ & $3.3396 \mathrm{e}-19$ & Reject \\
\hline topic1736 & 0.00014249 & 0.00012847 & $2.30916 \mathrm{e}+06$ & $5.447 \mathrm{e}-13$ & Reject \\
\hline topic1737 & 0.00011002 & $7.8551 \mathrm{e}-05$ & $2.1762 \mathrm{e}+06$ & $4.0551 \mathrm{e}-24$ & Reject \\
\hline topic1738 & 0.00012248 & $8.4292 \mathrm{e}-05$ & $1.74833 \mathrm{e}+06$ & $9.8038 \mathrm{e}-86$ & Reject \\
\hline topic1739 & $1.6602 \mathrm{e}-05$ & $2.6911 \mathrm{e}-05$ & $2.08412 \mathrm{e}+06$ & $2.0833 \mathrm{e}-25$ & Reject \\
\hline topic1740 & 0.0001261 & 0.00010222 & $2.37408 \mathrm{e}+06$ & $8.0909 \mathrm{e}-09$ & Reject \\
\hline topic1741 & 0.0001267 & $8.3412 \mathrm{e}-05$ & $2.1393 \mathrm{e}+06$ & $6.9191 \mathrm{e}-28$ & Reject \\
\hline topic1742 & 0.00014378 & $7.4213 \mathrm{e}-05$ & $1.47917 \mathrm{e}+06$ & $1.4938 \mathrm{e}-144$ & Reject \\
\hline topic1743 & 0.00012875 & 0.00010891 & $2.26599 \mathrm{e}+06$ & $2.6265 \mathrm{e}-16$ & Reject \\
\hline topic1744 & 0.00010223 & $7.4792 \mathrm{e}-05$ & $2.10818 \mathrm{e}+06$ & $2.6782 \mathrm{e}-31$ & Reject \\
\hline topic1745 & 0.00013107 & $9.2782 \mathrm{e}-05$ & $1.81672 \mathrm{e}+06$ & $1.6096 \mathrm{e}-73$ & Reject \\
\hline topic1746 & $3.6721 \mathrm{e}-05$ & $1.6474 \mathrm{e}-05$ & $1.63543 \mathrm{e}+06$ & $9.1925 \mathrm{e}-109$ & Reject \\
\hline topic1747 & 0.00013525 & 0.00013306 & $2.60816 \mathrm{e}+06$ & 0.35413 & Accept \\
\hline
\end{tabular}

Continued on next page 
Table A1 - Continued from previous page

\begin{tabular}{|c|c|c|c|c|c|}
\hline Feature & EN median & FL median & u-value & p-value & $\mathbf{H}_{0}$ \\
\hline topic1748 & $3.5325 \mathrm{e}-05$ & $1.3002 \mathrm{e}-05$ & $1.47463 \mathrm{e}+06$ & $2.8343 \mathrm{e}-142$ & Reject \\
\hline topic1749 & 0.00013117 & 0.00010065 & $2.2581 \mathrm{e}+06$ & 7.3176e-17 & Reject \\
\hline topic1750 & $9.6452 \mathrm{e}-05$ & $4.1008 \mathrm{e}-05$ & $1.4418 \mathrm{e}+06$ & $9.3202 \mathrm{e}-154$ & Reject \\
\hline topic1751 & 0.00021534 & 0.00018274 & $2.18171 \mathrm{e}+06$ & $8.9465 \mathrm{e}-24$ & Reject \\
\hline topic1752 & 0.00017186 & 0.00021929 & $2.13937 \mathrm{e}+06$ & $4.2387 \mathrm{e}-28$ & Reject \\
\hline topic1753 & 0.00010528 & $8.2197 \mathrm{e}-05$ & $2.1902 \mathrm{e}+06$ & $9.2157 \mathrm{e}-23$ & Reject \\
\hline topic1754 & $8.9198 \mathrm{e}-05$ & $5.3873 \mathrm{e}-05$ & $2.12113 \mathrm{e}+06$ & $1.2274 \mathrm{e}-29$ & Reject \\
\hline topic1755 & $9.821 \mathrm{e}-05$ & 0.00010251 & $2.61277 \mathrm{e}+06$ & 0.36366 & Accept \\
\hline topic1756 & 0.00015337 & 0.00010949 & $1.92891 \mathrm{e}+06$ & $1.3572 \mathrm{e}-54$ & Reject \\
\hline topic1757 & $2.4217 \mathrm{e}-05$ & $1.7939 \mathrm{e}-05$ & $2.33185 \mathrm{e}+06$ & $3.5864 \mathrm{e}-11$ & Reject \\
\hline topic1758 & 0.00011775 & $8.5005 \mathrm{e}-05$ & $1.89416 \mathrm{e}+06$ & $2.0575 \mathrm{e}-60$ & Reject \\
\hline topic1759 & 0.00010285 & $5.6436 \mathrm{e}-05$ & $1.43675 \mathrm{e}+06$ & $2.8021 \mathrm{e}-155$ & Reject \\
\hline topic1760 & $3.0809 \mathrm{e}-05$ & $1.8907 \mathrm{e}-05$ & $1.54678 \mathrm{e}+06$ & 7.5941e-129 & Reject \\
\hline topic1761 & 0.00013027 & 0.00012131 & $2.41858 \mathrm{e}+06$ & $2.0573 \mathrm{e}-06$ & Reject \\
\hline topic1762 & $8.5731 \mathrm{e}-05$ & $7.0058 \mathrm{e}-05$ & $2.25092 \mathrm{e}+06$ & $1.5684 \mathrm{e}-17$ & Reject \\
\hline topic1763 & $6.1559 \mathrm{e}-05$ & $5.9675 \mathrm{e}-05$ & $2.53978 \mathrm{e}+06$ & 0.024058 & Reject \\
\hline topic1764 & 0.00011734 & $5.0294 \mathrm{e}-05$ & $1.57798 \mathrm{e}+06$ & $4.2982 \mathrm{e}-121$ & Reject \\
\hline topic1765 & $8.9736 \mathrm{e}-05$ & $8.2767 \mathrm{e}-05$ & $2.47000 \mathrm{e}+06$ & 0.00027012 & Reject \\
\hline topic1766 & 0.00012507 & 0.00012003 & $2.52087 \mathrm{e}+06$ & 0.0094827 & Reject \\
\hline topic1767 & $1.1493 \mathrm{e}-05$ & $1.6325 \mathrm{e}-05$ & $2.31642 \mathrm{e}+06$ & $2.0655 \mathrm{e}-12$ & Reject \\
\hline topic1768 & 0.00013349 & 0.00011238 & $2.33186 \mathrm{e}+06$ & $2.227 \mathrm{e}-11$ & Reject \\
\hline topic1769 & 0.0001191 & 0.00015207 & $2.1886 \mathrm{e}+06$ & $8.168 \mathrm{e}-23$ & Reject \\
\hline topic1770 & 0.00012582 & $7.2404 \mathrm{e}-05$ & $1.5616 \mathrm{e}+06$ & $1.258 \mathrm{e}-124$ & Reject \\
\hline topic1771 & $3.9635 \mathrm{e}-05$ & $1.0958 \mathrm{e}-05$ & $1.49056 \mathrm{e}+06$ & $1.6147 \mathrm{e}-141$ & Reject \\
\hline topic1772 & $8.0904 \mathrm{e}-05$ & $8.068 \mathrm{e}-05$ & $2.62054 \mathrm{e}+06$ & 0.4295 & Accept \\
\hline topic1773 & $8.5792 \mathrm{e}-05$ & $4.1018 \mathrm{e}-05$ & $1.48803 \mathrm{e}+06$ & $2.4226 \mathrm{e}-142$ & Reject \\
\hline topic1774 & $3.503 \mathrm{e}-05$ & $8.4333 \mathrm{e}-06$ & $1.10132 \mathrm{e}+06$ & $6.1467 \mathrm{e}-253$ & Reject \\
\hline topic1775 & 0.00010929 & 0.00011432 & $2.55303 \mathrm{e}+06$ & 0.046184 & Reject \\
\hline topic1776 & 0.00015583 & 0.00015876 & $2.62219 \mathrm{e}+06$ & 0.43435 & Accept \\
\hline topic1777 & $5.2639 \mathrm{e}-05$ & $2.9534 \mathrm{e}-05$ & $1.90389 \mathrm{e}+06$ & $1.1453 \mathrm{e}-57$ & Reject \\
\hline topic1778 & 0.0001218 & $3.3248 \mathrm{e}-05$ & $1.35277 \mathrm{e}+06$ & $1.2695 \mathrm{e}-176$ & Reject \\
\hline topic1779 & 0.00010901 & $7.5318 \mathrm{e}-05$ & $2.10404 \mathrm{e}+06$ & $6.946 \mathrm{e}-32$ & Reject \\
\hline topic1780 & $8.9199 \mathrm{e}-05$ & 0.00014377 & 103196 & $1.8557 \mathrm{e}-10$ & Reject \\
\hline topic1781 & 0.00014646 & $9.8551 \mathrm{e}-05$ & $1.96473 \mathrm{e}+06$ & $1.9557 \mathrm{e}-49$ & Reject \\
\hline topic1782 & 0.00011428 & $5.4916 \mathrm{e}-05$ & $1.65186 \mathrm{e}+06$ & $5.5652 \mathrm{e}-104$ & Reject \\
\hline topic1783 & $6.4188 \mathrm{e}-05$ & $2.5516 \mathrm{e}-05$ & $1.37484 \mathrm{e}+06$ & $4.9452 \mathrm{e}-172$ & Reject \\
\hline topic1784 & $5.4566 \mathrm{e}-05$ & $2.4982 \mathrm{e}-05$ & $1.66915 \mathrm{e}+06$ & 8.2492e-102 & Reject \\
\hline topic1785 & $7.3599 \mathrm{e}-05$ & $3.7734 \mathrm{e}-05$ & $2.02561 \mathrm{e}+06$ & $4.1245 \mathrm{e}-41$ & Reject \\
\hline topic1786 & $4.2087 \mathrm{e}-05$ & $2.1728 \mathrm{e}-05$ & $1.84377 \mathrm{e}+06$ & $5.7771 \mathrm{e}-66$ & Reject \\
\hline topic1787 & $2.9941 \mathrm{e}-05$ & $1.5871 \mathrm{e}-05$ & $1.62656 \mathrm{e}+06$ & 7.3007e-111 & Reject \\
\hline topic1788 & 0.00013701 & 0.00013015 & $2.5258 \mathrm{e}+06$ & 0.013471 & Reject \\
\hline topic1789 & $9.5753 \mathrm{e}-05$ & $8.1995 \mathrm{e}-05$ & $2.41633 \mathrm{e}+06$ & $9.9657 \mathrm{e}-07$ & Reject \\
\hline
\end{tabular}


Table A1 - Continued from previous page

\begin{tabular}{|c|c|c|c|c|c|}
\hline Feature & EN median & FL median & u-value & p-value & $\mathrm{H}_{\mathbf{0}}$ \\
\hline topic1790 & 0.00013581 & 0.00010304 & $2.17617 \mathrm{e}+06$ & $2.5541 \mathrm{e}-24$ & Reject \\
\hline topic1791 & 0.00012963 & 0.0001161 & $2.46851 \mathrm{e}+06$ & 0.00016464 & Reject \\
\hline topic1792 & 0.00017213 & 0.00020413 & $2.29213 \mathrm{e}+06$ & $3.8807 \mathrm{e}-14$ & Reject \\
\hline topic1793 & $6.9616 \mathrm{e}-05$ & $9.848 \mathrm{e}-05$ & $1.91994 \mathrm{e}+06$ & $1.6099 \mathrm{e}-56$ & Reject \\
\hline topic1794 & 0.00010149 & $8.0269 \mathrm{e}-05$ & $2.11259 \mathrm{e}+06$ & $1.0426 \mathrm{e}-30$ & Reject \\
\hline topic1795 & $9.2268 \mathrm{e}-05$ & 0.00015117 & $1.77945 \mathrm{e}+06$ & $2.1749 \mathrm{e}-80$ & Reject \\
\hline topic1796 & $7.5302 \mathrm{e}-05$ & $2.7355 \mathrm{e}-05$ & $1.26194 \mathrm{e}+06$ & $1.9525 \mathrm{e}-204$ & Reject \\
\hline topic1797 & 0.00017248 & 0.00010966 & $1.99498 \mathrm{e}+06$ & $2.4978 \mathrm{e}-45$ & Reject \\
\hline topic1798 & 0.00015313 & 0.00013399 & $2.22392 \mathrm{e}+06$ & $9.2631 \mathrm{e}-20$ & Reject \\
\hline topic1799 & 0.00014523 & 0.00012563 & $2.36742 \mathrm{e}+06$ & $3.9077 \mathrm{e}-09$ & Reject \\
\hline topic1800 & $2.5321 \mathrm{e}-05$ & $1.9053 \mathrm{e}-05$ & $2.47592 \mathrm{e}+06$ & 0.00036944 & Reject \\
\hline topic1801 & 0.00013141 & $9.8438 \mathrm{e}-05$ & $2.22986 \mathrm{e}+06$ & $2.0261 \mathrm{e}-18$ & Reject \\
\hline topic1802 & 0.00013812 & $7.6903 \mathrm{e}-05$ & $1.96943 \mathrm{e}+06$ & $6.4661 \mathrm{e}-49$ & Reject \\
\hline topic1803 & 0.00014366 & $9.8484 \mathrm{e}-05$ & $1.82215 \mathrm{e}+06$ & $2.0143 \mathrm{e}-72$ & Reject \\
\hline topic1804 & 0.00010079 & $8.2782 \mathrm{e}-05$ & $2.29492 \mathrm{e}+06$ & $5.1786 \mathrm{e}-14$ & Reject \\
\hline topic1805 & 0.00013446 & 0.00011738 & $2.27247 \mathrm{e}+06$ & $8.5367 \mathrm{e}-16$ & Reject \\
\hline topic1806 & 0.00013807 & 0.00011291 & $2.34561 \mathrm{e}+06$ & $1.9635 \mathrm{e}-10$ & Reject \\
\hline topic1807 & 0.00011431 & 0.00010151 & $2.37593 \mathrm{e}+06$ & $1.8167 \mathrm{e}-08$ & Reject \\
\hline topic1808 & $2.2016 \mathrm{e}-05$ & $5.0704 \mathrm{e}-05$ & $1.95519 \mathrm{e}+06$ & $1.2768 \mathrm{e}-34$ & Reject \\
\hline topic1809 & $7.0444 \mathrm{e}-05$ & $6.4269 \mathrm{e}-05$ & $2.40678 \mathrm{e}+06$ & $3.3964 \mathrm{e}-07$ & Reject \\
\hline topic1810 & 0.00014534 & 0.00011233 & $2.07464 \mathrm{e}+06$ & $3.2103 \mathrm{e}-35$ & Reject \\
\hline topic1811 & 0.00012923 & 0.00011087 & $2.15385 \mathrm{e}+06$ & $1.8067 \mathrm{e}-26$ & Reject \\
\hline topic1812 & $9.1506 \mathrm{e}-05$ & $4.9376 \mathrm{e}-05$ & $1.59206 \mathrm{e}+06$ & $7.0056 \mathrm{e}-118$ & Reject \\
\hline topic1813 & 0.00011056 & $4.4265 \mathrm{e}-05$ & $1.43855 \mathrm{e}+06$ & $1.4617 \mathrm{e}-152$ & Reject \\
\hline topic1814 & $8.114 \mathrm{e}-05$ & $4.7114 \mathrm{e}-05$ & $1.78866 \mathrm{e}+06$ & $1.0513 \mathrm{e}-78$ & Reject \\
\hline topic1815 & 0.00014731 & 0.00010862 & $2.15099 \mathrm{e}+06$ & $1.9191 \mathrm{e}-26$ & Reject \\
\hline topic1816 & $5.6755 \mathrm{e}-05$ & $5.0275 \mathrm{e}-05$ & $2.3495 \mathrm{e}+06$ & $2.4958 \mathrm{e}-10$ & Reject \\
\hline topic1817 & $6.3439 \mathrm{e}-06$ & $2.4395 \mathrm{e}-05$ & $1.51193 \mathrm{e}+06$ & $7.7808 \mathrm{e}-128$ & Reject \\
\hline topic1818 & $5.072 \mathrm{e}-05$ & $3.359 \mathrm{e}-05$ & $2.10657 \mathrm{e}+06$ & $3.7279 \mathrm{e}-31$ & Reject \\
\hline topic1819 & 0.00011664 & $7.7422 \mathrm{e}-05$ & $1.7953 \mathrm{e}+06$ & $1.6803 \mathrm{e}-77$ & Reject \\
\hline topic1820 & $8.7383 \mathrm{e}-05$ & $7.2792 \mathrm{e}-05$ & $2.42283 \mathrm{e}+06$ & $4.4641 \mathrm{e}-06$ & Reject \\
\hline topic1821 & $4.5015 \mathrm{e}-05$ & $3.1153 \mathrm{e}-05$ & $2.25803 \mathrm{e}+06$ & $8.8251 \mathrm{e}-17$ & Reject \\
\hline topic1822 & $9.1123 \mathrm{e}-05$ & $7.6887 \mathrm{e}-05$ & $2.42893 \mathrm{e}+06$ & $3.8511 \mathrm{e}-06$ & Reject \\
\hline topic1823 & 0.00012883 & $7.4185 \mathrm{e}-05$ & $1.73205 \mathrm{e}+06$ & $1.1731 \mathrm{e}-88$ & Reject \\
\hline topic1824 & 0.00013539 & $8.6501 \mathrm{e}-05$ & $1.83504 \mathrm{e}+06$ & $4.9762 \mathrm{e}-70$ & Reject \\
\hline topic1825 & 0.00010259 & $7.0501 \mathrm{e}-05$ & $2.10842 \mathrm{e}+06$ & $2.1307 \mathrm{e}-31$ & Reject \\
\hline topic1826 & 0.00015705 & 0.00016128 & $2.57545 \mathrm{e}+06$ & 0.11882 & Accept \\
\hline topic1827 & 0.00015102 & 0.00010217 & $2.02376 \mathrm{e}+06$ & $2.2977 \mathrm{e}-41$ & Reject \\
\hline topic1828 & 0.00010357 & 0.00010537 & $2.59027 \mathrm{e}+06$ & 0.19024 & Accept \\
\hline topic1829 & $2.1528 \mathrm{e}-05$ & $1.0898 \mathrm{e}-05$ & $1.96431 \mathrm{e}+06$ & $4.9716 \mathrm{e}-42$ & Reject \\
\hline topic1830 & 0.00011316 & 0.0001124 & $2.59167 \mathrm{e}+06$ & 0.22831 & Accept \\
\hline topic1831 & 0.00012702 & $9.507 \mathrm{e}-05$ & $2.0266 \mathrm{e}+06$ & $3.136 \mathrm{e}-41$ & Reject \\
\hline
\end{tabular}


Table A1 - Continued from previous page

\begin{tabular}{|c|c|c|c|c|c|}
\hline Feature & EN median & FL median & u-value & p-value & $\mathbf{H}_{0}$ \\
\hline topic1832 & $1.9149 \mathrm{e}-05$ & $3.1561 \mathrm{e}-05$ & $2.1944 \mathrm{e}+06$ & $5.7455 \mathrm{e}-22$ & Reject \\
\hline topic1833 & 0.00015343 & 0.0001656 & $2.46094 \mathrm{e}+06$ & $8.5037 \mathrm{e}-05$ & Reject \\
\hline topic1834 & $9.1235 \mathrm{e}-05$ & $6.3067 \mathrm{e}-05$ & $2.15814 \mathrm{e}+06$ & $1.0166 \mathrm{e}-25$ & Reject \\
\hline topic1835 & $9.8533 \mathrm{e}-05$ & $8.9341 \mathrm{e}-05$ & $2.50092 \mathrm{e}+06$ & 0.0022352 & Reject \\
\hline topic1836 & 0.00012246 & 0.00012644 & $2.53752 \mathrm{e}+06$ & 0.020049 & Reject \\
\hline topic1837 & $1.031 \mathrm{e}-05$ & $1.9541 \mathrm{e}-05$ & $1.96939 \mathrm{e}+06$ & $3.2607 \mathrm{e}-43$ & Reject \\
\hline topic1838 & $1.1291 \mathrm{e}-05$ & $1.0389 \mathrm{e}-05$ & $2.55141 \mathrm{e}+06$ & 0.13687 & Accept \\
\hline topic1839 & 0.00020795 & 0.00017015 & $2.14283 \mathrm{e}+06$ & $9.8784 \mathrm{e}-28$ & Reject \\
\hline topic1840 & $7.5457 \mathrm{e}-05$ & $6.2655 \mathrm{e}-05$ & $2.34394 \mathrm{e}+06$ & $9.582 \mathrm{e}-11$ & Reject \\
\hline topic1841 & $2.19 \mathrm{e}-05$ & $5.9402 \mathrm{e}-05$ & $1.69912 \mathrm{e}+06$ & $1.0589 \mathrm{e}-95$ & Reject \\
\hline topic1842 & 0.00012915 & 0.00012727 & $2.57026 \mathrm{e}+06$ & 0.092917 & Accept \\
\hline topic1843 & 0.00012693 & 0.00016538 & $2.18427 \mathrm{e}+06$ & $2.482 \mathrm{e}-23$ & Reject \\
\hline topic1844 & 0.00012313 & 0.00011855 & $2.51426 \mathrm{e}+06$ & 0.0050679 & Reject \\
\hline topic1845 & $7.2131 \mathrm{e}-05$ & $4.5611 \mathrm{e}-05$ & $1.76035 \mathrm{e}+06$ & $1.2836 \mathrm{e}-83$ & Reject \\
\hline topic1846 & $9.4485 \mathrm{e}-05$ & $6.049 \mathrm{e}-05$ & $2.08192 \mathrm{e}+06$ & $1.3995 \mathrm{e}-34$ & Reject \\
\hline topic1847 & $3.9253 \mathrm{e}-05$ & $2.475 \mathrm{e}-05$ & $1.78481 \mathrm{e}+06$ & $4.19 \mathrm{e}-79$ & Reject \\
\hline topic1848 & 0.00015126 & 0.00012697 & $2.24908 \mathrm{e}+06$ & $1.6614 \mathrm{e}-17$ & Reject \\
\hline topic1849 & $4.7443 \mathrm{e}-05$ & $1.4363 \mathrm{e}-05$ & $1.28686 \mathrm{e}+06$ & $9.4888 \mathrm{e}-197$ & Reject \\
\hline topic1850 & $9.424 \mathrm{e}-05$ & $4.6101 \mathrm{e}-05$ & $1.8938 \mathrm{e}+06$ & $9.2491 \mathrm{e}-61$ & Reject \\
\hline topic1851 & $3.3978 \mathrm{e}-05$ & $1.5254 \mathrm{e}-05$ & $1.92 \mathrm{e}+06$ & $7.4328 \mathrm{e}-53$ & Reject \\
\hline topic1852 & $9.177 \mathrm{e}-05$ & 0.00012683 & $2.03740 \mathrm{e}+06$ & $4.3396 \mathrm{e}-40$ & Reject \\
\hline topic1853 & 0.00011772 & $7.5703 \mathrm{e}-05$ & $2.02351 \mathrm{e}+06$ & $3.8823 \mathrm{e}-41$ & Reject \\
\hline topic1854 & $6.9455 \mathrm{e}-05$ & $4.3054 \mathrm{e}-05$ & $1.81462 \mathrm{e}+06$ & $6.8712 \mathrm{e}-74$ & Reject \\
\hline topic1855 & 0.00012475 & $5.7642 \mathrm{e}-05$ & $1.557 \mathrm{e}+06$ & $4.4712 \mathrm{e}-126$ & Reject \\
\hline topic1856 & 0.00015457 & 0.00012792 & $2.29396 \mathrm{e}+06$ & $4.3278 \mathrm{e}-14$ & Reject \\
\hline topic1857 & $8.4088 \mathrm{e}-05$ & $6.4037 \mathrm{e}-05$ & $2.25642 \mathrm{e}+06$ & $5.4323 \mathrm{e}-17$ & Reject \\
\hline topic1858 & $9.3418 \mathrm{e}-05$ & $4.368 \mathrm{e}-05$ & $1.64827 \mathrm{e}+06$ & $7.3948 \mathrm{e}-106$ & Reject \\
\hline topic1859 & $9.4457 \mathrm{e}-05$ & $4.6411 \mathrm{e}-05$ & $1.41673 \mathrm{e}+06$ & $6.8728 \mathrm{e}-161$ & Reject \\
\hline topic1860 & $5.4603 \mathrm{e}-05$ & $9.9383 \mathrm{e}-06$ & $1.7564 \mathrm{e}+06$ & $1.0833 \mathrm{e}-79$ & Reject \\
\hline topic1861 & $8.4731 \mathrm{e}-05$ & $5.0084 \mathrm{e}-05$ & $1.68197 \mathrm{e}+06$ & $8.3209 \mathrm{e}-99$ & Reject \\
\hline topic1862 & 0.00013548 & $7.9346 \mathrm{e}-05$ & $1.62179 \mathrm{e}+06$ & $2.4331 \mathrm{e}-111$ & Reject \\
\hline topic1863 & $7.1746 \mathrm{e}-05$ & $6.3763 \mathrm{e}-05$ & $2.5169 \mathrm{e}+06$ & 0.0084866 & Reject \\
\hline topic1864 & 0.00012731 & $8.8266 \mathrm{e}-05$ & $1.98814 \mathrm{e}+06$ & $7.0812 \mathrm{e}-46$ & Reject \\
\hline topic1865 & $4.9031 \mathrm{e}-05$ & $3.2876 \mathrm{e}-05$ & $1.91078 \mathrm{e}+06$ & $6.171 \mathrm{e}-58$ & Reject \\
\hline topic1866 & $5.8559 \mathrm{e}-05$ & $6.3932 \mathrm{e}-05$ & $2.50969 \mathrm{e}+06$ & 0.0037577 & Reject \\
\hline topic1867 & $1.9298 \mathrm{e}-05$ & $1.2359 \mathrm{e}-05$ & $1.78591 \mathrm{e}+06$ & $7.152 \mathrm{e}-79$ & Reject \\
\hline topic1868 & $2.8034 \mathrm{e}-05$ & $8.6433 \mathrm{e}-06$ & $1.26493 \mathrm{e}+06$ & $2.3443 \mathrm{e}-203$ & Reject \\
\hline topic1869 & $9.7743 \mathrm{e}-05$ & $8.418 \mathrm{e}-05$ & $2.39914 \mathrm{e}+06$ & $1.3936 \mathrm{e}-07$ & Reject \\
\hline topic1870 & 0.0001992 & 0.00013009 & $1.54535 \mathrm{e}+06$ & $3.53 \mathrm{e}-129$ & Reject \\
\hline topic1871 & $4.9447 \mathrm{e}-05$ & $3.5082 \mathrm{e}-05$ & $2.10196 \mathrm{e}+06$ & $6.8995 \mathrm{e}-31$ & Reject \\
\hline topic1872 & 0.0001264 & $8.3799 \mathrm{e}-05$ & $1.75027 \mathrm{e}+06$ & $1.119 \mathrm{e}-85$ & Reject \\
\hline topic1873 & 0.00015931 & 0.00020106 & $2.28806 \mathrm{e}+06$ & $1.3346 \mathrm{e}-14$ & Reject \\
\hline
\end{tabular}


Table A1 - Continued from previous page

\begin{tabular}{|c|c|c|c|c|c|}
\hline Feature & EN median & FL median & u-value & p-value & $\mathbf{H}_{0}$ \\
\hline topic1874 & $3.7938 \mathrm{e}-05$ & $1.9747 \mathrm{e}-05$ & $1.4313 \mathrm{e}+06$ & $2.7818 \mathrm{e}-157$ & Reject \\
\hline topic1875 & 0.0001399 & 0.00014334 & $2.5462 \mathrm{e}+06$ & 0.033215 & Reject \\
\hline topic1876 & 0.00015609 & 0.0001079 & $2.4382 \mathrm{e}+06$ & $3.903 \mathrm{e}-05$ & Reject \\
\hline topic1877 & 0.00014267 & 0.00011529 & $2.23036 \mathrm{e}+06$ & 7.6722e-19 & Reject \\
\hline topic1878 & $9.0405 \mathrm{e}-05$ & 0.00010544 & $2.38936 \mathrm{e}+06$ & $6.2888 \mathrm{e}-08$ & Reject \\
\hline topic1879 & 0.00011442 & $6.2738 \mathrm{e}-05$ & $1.56822 \mathrm{e}+06$ & $1.7895 \mathrm{e}-123$ & Reject \\
\hline topic1880 & 0.0001252 & 0.00013916 & $2.49031 \mathrm{e}+06$ & 0.0011302 & Reject \\
\hline topic1881 & 0.00012418 & $7.3476 \mathrm{e}-05$ & $1.72558 \mathrm{e}+06$ & $6.2423 \mathrm{e}-90$ & Reject \\
\hline topic1882 & $9.7227 \mathrm{e}-05$ & $3.0217 \mathrm{e}-05$ & $1.87124 \mathrm{e}+06$ & $1.8965 \mathrm{e}-62$ & Reject \\
\hline topic1883 & $7.7423 \mathrm{e}-05$ & $7.7141 \mathrm{e}-05$ & $2.61917 \mathrm{e}+06$ & 0.41747 & Accept \\
\hline topic1884 & 0.00013413 & 0.00010206 & $2.14457 \mathrm{e}+06$ & $1.9395 \mathrm{e}-27$ & Reject \\
\hline topic1885 & 0.00033621 & 0.0002604 & $1.9004 \mathrm{e}+06$ & $1.0336 \mathrm{e}-59$ & Reject \\
\hline topic1886 & 0.00018958 & 0.00014081 & $1.90554 \mathrm{e}+06$ & $9.1181 \mathrm{e}-59$ & Reject \\
\hline topic1887 & $8.8098 \mathrm{e}-05$ & $9.8326 \mathrm{e}-05$ & $2.43201 \mathrm{e}+06$ & $5.2985 \mathrm{e}-06$ & Reject \\
\hline topic1888 & $9.4838 \mathrm{e}-05$ & $3.931 \mathrm{e}-05$ & $1.69027 \mathrm{e}+06$ & $2.7131 \mathrm{e}-97$ & Reject \\
\hline topic1889 & 0.00011672 & 0.00010308 & $2.49828 \mathrm{e}+06$ & 0.0055984 & Reject \\
\hline topic1890 & 0.00013134 & $9.7773 \mathrm{e}-05$ & $1.98764 \mathrm{e}+06$ & $1.2976 \mathrm{e}-46$ & Reject \\
\hline topic1891 & 0.00015042 & 0.00013567 & $2.41040 \mathrm{e}+06$ & $5.1379 \mathrm{e}-07$ & Reject \\
\hline topic1892 & $7.7582 \mathrm{e}-05$ & 0.0001061 & $2.202 \mathrm{e}+06$ & $9.6792 \mathrm{e}-22$ & Reject \\
\hline topic1893 & 0.0001062 & $6.1442 \mathrm{e}-05$ & $2.06188 \mathrm{e}+06$ & $5.7853 \mathrm{e}-35$ & Reject \\
\hline topic1894 & 0.00014291 & $9.3573 \mathrm{e}-05$ & $1.97246 \mathrm{e}+06$ & $6.8883 \mathrm{e}-49$ & Reject \\
\hline topic1895 & 0.00017972 & 0.00014863 & $2.27068 \mathrm{e}+06$ & $8.9533 \mathrm{e}-16$ & Reject \\
\hline topic1896 & $8.4635 \mathrm{e}-05$ & $5.7217 \mathrm{e}-05$ & $1.73717 \mathrm{e}+06$ & $3.3425 \mathrm{e}-88$ & Reject \\
\hline topic1897 & $3.3105 \mathrm{e}-05$ & $1.9447 \mathrm{e}-05$ & $1.59273 \mathrm{e}+06$ & $1.7078 \mathrm{e}-118$ & Reject \\
\hline topic1898 & $7.5706 \mathrm{e}-05$ & $2.309 \mathrm{e}-05$ & 801742 & 0 & Reject \\
\hline topic1899 & $5.2596 \mathrm{e}-05$ & $2.1272 \mathrm{e}-05$ & $1.66515 \mathrm{e}+06$ & $8.7717 \mathrm{e}-102$ & Reject \\
\hline topic1900 & $3.0871 \mathrm{e}-05$ & $1.4089 \mathrm{e}-05$ & $1.68865 \mathrm{e}+06$ & $2.7887 \mathrm{e}-97$ & Reject \\
\hline topic1901 & 0.00011377 & $9.0059 \mathrm{e}-05$ & $2.21014 \mathrm{e}+06$ & $1.0202 \mathrm{e}-20$ & Reject \\
\hline topic1902 & 0.00023952 & 0.00027688 & $2.43004 \mathrm{e}+06$ & $6.7424 \mathrm{e}-06$ & Reject \\
\hline topic1903 & $8.0058 \mathrm{e}-05$ & $8.3899 \mathrm{e}-05$ & $2.50426 \mathrm{e}+06$ & 0.0026008 & Reject \\
\hline topic1904 & 0.0001412 & 0.00013175 & $2.45429 \mathrm{e}+06$ & $4.6528 \mathrm{e}-05$ & Reject \\
\hline topic1905 & $8.9809 \mathrm{e}-05$ & 0.00011557 & $2.26994 \mathrm{e}+06$ & $5.3972 \mathrm{e}-16$ & Reject \\
\hline topic1906 & 0.00024619 & 0.00024841 & $2.60132 \mathrm{e}+06$ & 0.2729 & Accept \\
\hline topic1907 & 0.00012702 & 0.00012723 & $2.57797 \mathrm{e}+06$ & 0.12486 & Accept \\
\hline topic1908 & 0.00014371 & 0.00012029 & $2.19058 \mathrm{e}+06$ & $8.1088 \mathrm{e}-23$ & Reject \\
\hline topic1909 & $7.0099 \mathrm{e}-05$ & $2.6868 \mathrm{e}-05$ & 972529 & $1.634 \mathrm{e}-298$ & Reject \\
\hline topic1910 & $9.7043 \mathrm{e}-05$ & $7.5753 \mathrm{e}-05$ & $2.2177 \mathrm{e}+06$ & $3.9304 \mathrm{e}-20$ & Reject \\
\hline topic1911 & $5.9547 \mathrm{e}-05$ & 0.00021324 & $1.45934 \mathrm{e}+06$ & $7.7337 \mathrm{e}-140$ & Reject \\
\hline topic1912 & $6.1291 \mathrm{e}-05$ & $3.5298 \mathrm{e}-05$ & $2.17852 \mathrm{e}+06$ & $2.6321 \mathrm{e}-23$ & Reject \\
\hline topic1913 & $9.824 \mathrm{e}-05$ & $7.3117 \mathrm{e}-05$ & $2.31418 \mathrm{e}+06$ & $2.3606 \mathrm{e}-12$ & Reject \\
\hline topic1914 & 0.00010404 & $9.4702 \mathrm{e}-05$ & $2.43534 \mathrm{e}+06$ & 7.437e-06 & Reject \\
\hline topic1915 & $8.8059 \mathrm{e}-05$ & $8.4903 \mathrm{e}-05$ & $2.60187 \mathrm{e}+06$ & 0.27621 & Accept \\
\hline
\end{tabular}


Table A1 - Continued from previous page

\begin{tabular}{|c|c|c|c|c|c|}
\hline Feature & EN median & FL median & u-value & p-value & $\mathbf{H}_{0}$ \\
\hline topic1916 & 0.00010596 & 0.00012125 & $2.39503 \mathrm{e}+06$ & $1.1144 \mathrm{e}-07$ & Reject \\
\hline topic1917 & 0.00014094 & $9.7017 \mathrm{e}-05$ & $2.00159 \mathrm{e}+06$ & $1.4634 \mathrm{e}-44$ & Reject \\
\hline topic1918 & 0.00014258 & $9.058 \mathrm{e}-05$ & $1.69071 \mathrm{e}+06$ & $1.0984 \mathrm{e}-96$ & Reject \\
\hline topic1919 & $8.5467 \mathrm{e}-05$ & $7.5575 \mathrm{e}-05$ & $2.44449 \mathrm{e}+06$ & $2.2919 \mathrm{e}-05$ & Reject \\
\hline topic1920 & 0.00010637 & $6.7687 \mathrm{e}-05$ & $1.77986 \mathrm{e}+06$ & $7.9158 \mathrm{e}-80$ & Reject \\
\hline topic1921 & 0.00015518 & 0.00012552 & $2.12401 \mathrm{e}+06$ & $1.166 \mathrm{e}-29$ & Reject \\
\hline topic1922 & 0.00011038 & $8.3488 \mathrm{e}-05$ & $2.33451 \mathrm{e}+06$ & $3.2614 \mathrm{e}-11$ & Reject \\
\hline topic1923 & $7.6605 \mathrm{e}-05$ & $6.867 \mathrm{e}-05$ & $2.44301 \mathrm{e}+06$ & $1.7805 \mathrm{e}-05$ & Reject \\
\hline topic1924 & $6.1061 \mathrm{e}-05$ & $1.058 \mathrm{e}-05$ & $1.08622 \mathrm{e}+06$ & $1.275 \mathrm{e}-258$ & Reject \\
\hline topic1925 & $5.359 \mathrm{e}-06$ & $4.7687 \mathrm{e}-06$ & $2.42545 \mathrm{e}+06$ & 0.0055845 & Reject \\
\hline topic1926 & 0.00020425 & 0.00024073 & $2.28147 \mathrm{e}+06$ & $5.038 \mathrm{e}-15$ & Reject \\
\hline topic1927 & 0.0001482 & 0.00014224 & $2.56446 \mathrm{e}+06$ & 0.084448 & Accept \\
\hline topic1928 & $9.5769 \mathrm{e}-05$ & $7.4047 \mathrm{e}-05$ & $2.29691 \mathrm{e}+06$ & $1.0247 \mathrm{e}-13$ & Reject \\
\hline topic1929 & 0.00013349 & 0.00013858 & $2.45719 \mathrm{e}+06$ & $6.0655 \mathrm{e}-05$ & Reject \\
\hline topic1930 & 0.00010484 & $8.6672 \mathrm{e}-05$ & $2.24214 \mathrm{e}+06$ & $4.3712 \mathrm{e}-18$ & Reject \\
\hline topic1931 & 0.00013027 & $8.1072 \mathrm{e}-05$ & $1.80912 \mathrm{e}+06$ & $3.1808 \mathrm{e}-74$ & Reject \\
\hline topic1932 & 0.00012484 & $6.9297 \mathrm{e}-05$ & $1.71084 \mathrm{e}+06$ & $5.076 \mathrm{e}-93$ & Reject \\
\hline topic1933 & 0.00015989 & 0.00011434 & $1.91552 \mathrm{e}+06$ & $4.7183 \mathrm{e}-57$ & Reject \\
\hline topic1934 & 0.00011974 & $9.5259 \mathrm{e}-05$ & $2.02473 \mathrm{e}+06$ & $1.3241 \mathrm{e}-41$ & Reject \\
\hline topic1935 & 0.00012624 & $8.1497 \mathrm{e}-05$ & $2.08628 \mathrm{e}+06$ & $7.7842 \mathrm{e}-34$ & Reject \\
\hline topic1936 & $6.2192 \mathrm{e}-05$ & $8.1311 \mathrm{e}-05$ & $2.25256 \mathrm{e}+06$ & $4.8231 \mathrm{e}-17$ & Reject \\
\hline topic1937 & $8.7099 \mathrm{e}-05$ & $9.0362 \mathrm{e}-05$ & $2.55136 \mathrm{e}+06$ & 0.047876 & Reject \\
\hline topic1938 & 0.00013575 & $6.5263 \mathrm{e}-05$ & $1.55783 \mathrm{e}+06$ & $6.9332 \mathrm{e}-126$ & Reject \\
\hline topic1939 & 0.00012272 & 0.00011058 & $2.55167 \mathrm{e}+06$ & 0.041171 & Reject \\
\hline topic1940 & $3.5067 \mathrm{e}-05$ & $2.221 \mathrm{e}-05$ & $2.28426 \mathrm{e}+06$ & $9.8937 \mathrm{e}-15$ & Reject \\
\hline topic1941 & 0.00012125 & 0.00011279 & $2.57968 \mathrm{e}+06$ & 0.15631 & Accept \\
\hline topic1942 & $6.9629 \mathrm{e}-05$ & $6.5217 \mathrm{e}-05$ & $2.49462 \mathrm{e}+06$ & 0.0018289 & Reject \\
\hline topic1943 & $3.0366 \mathrm{e}-05$ & $2.1999 \mathrm{e}-05$ & $2.20469 \mathrm{e}+06$ & $1.72 \mathrm{e}-21$ & Reject \\
\hline topic1944 & $1.1577 \mathrm{e}-05$ & $2.0702 \mathrm{e}-05$ & $2.00898 \mathrm{e}+06$ & $6.3383 \mathrm{e}-40$ & Reject \\
\hline topic1945 & 0.00015234 & 0.00012438 & $2.27971 \mathrm{e}+06$ & $4.4708 \mathrm{e}-15$ & Reject \\
\hline topic1946 & $5.6514 \mathrm{e}-05$ & $4.2778 \mathrm{e}-05$ & $2.10046 \mathrm{e}+06$ & $1.202 \mathrm{e}-31$ & Reject \\
\hline topic1947 & $2.5498 \mathrm{e}-05$ & $6.0245 \mathrm{e}-06$ & $1.03799 \mathrm{e}+06$ & $1.2188 \mathrm{e}-274$ & Reject \\
\hline topic1948 & 0.00015386 & 0.00015453 & $2.61010 \mathrm{e}+06$ & 0.33186 & Accept \\
\hline topic1949 & $7.3499 \mathrm{e}-05$ & $5.3118 \mathrm{e}-05$ & $2.02674 \mathrm{e}+06$ & $3.1849 \mathrm{e}-41$ & Reject \\
\hline topic1950 & $8.9294 \mathrm{e}-05$ & $4.0184 \mathrm{e}-05$ & $1.49274 \mathrm{e}+06$ & $3.4951 \mathrm{e}-141$ & Reject \\
\hline topic1951 & 0.00015629 & 0.00013897 & $2.47169 \mathrm{e}+06$ & 0.00048935 & Reject \\
\hline topic1952 & 0.00015023 & $8.8149 \mathrm{e}-05$ & $1.68780 \mathrm{e}+06$ & $1.8765 \mathrm{e}-97$ & Reject \\
\hline topic1953 & 0.00015572 & 0.00012511 & $2.20042 \mathrm{e}+06$ & $8.4802 \mathrm{e}-22$ & Reject \\
\hline topic1954 & $9.6457 \mathrm{e}-05$ & $4.386 \mathrm{e}-05$ & $1.82051 \mathrm{e}+06$ & $2.2707 \mathrm{e}-72$ & Reject \\
\hline topic1955 & $9.1242 \mathrm{e}-05$ & $2.6056 \mathrm{e}-05$ & $1.6971 \mathrm{e}+06$ & $9.7984 \mathrm{e}-96$ & Reject \\
\hline topic1956 & $6.282 \mathrm{e}-05$ & $1.4559 \mathrm{e}-05$ & $1.05331 \mathrm{e}+06$ & $1.8755 \mathrm{e}-269$ & Reject \\
\hline topic1957 & $6.2023 \mathrm{e}-06$ & $2.7806 \mathrm{e}-05$ & $1.64827 \mathrm{e}+06$ & $4.3769 \mathrm{e}-32$ & Reject \\
\hline
\end{tabular}


Table A1 - Continued from previous page

\begin{tabular}{|c|c|c|c|c|c|}
\hline Feature & EN median & FL median & u-value & p-value & $\mathbf{H}_{0}$ \\
\hline topic1958 & 0.00020408 & 0.00015501 & $1.88522 \mathrm{e}+06$ & $3.909 \mathrm{e}-62$ & Reject \\
\hline topic1959 & $8.5876 \mathrm{e}-05$ & $7.0646 \mathrm{e}-05$ & $2.25229 \mathrm{e}+06$ & $3.0018 \mathrm{e}-17$ & Reject \\
\hline topic1960 & $1.5997 \mathrm{e}-06$ & $8.9118 \mathrm{e}-06$ & $1.67788 \mathrm{e}+06$ & $2.7359 \mathrm{e}-64$ & Reject \\
\hline topic1961 & 0.00015774 & 0.00011817 & $1.98554 \mathrm{e}+06$ & $8.7945 \mathrm{e}-47$ & Reject \\
\hline topic1962 & $6.6562 \mathrm{e}-05$ & $4.6436 \mathrm{e}-05$ & $1.98485 \mathrm{e}+06$ & $6.0069 \mathrm{e}-46$ & Reject \\
\hline topic1963 & 0.00010747 & $6.7012 \mathrm{e}-05$ & $2.14721 \mathrm{e}+06$ & $9.7484 \mathrm{e}-27$ & Reject \\
\hline topic1964 & $7.1466 \mathrm{e}-05$ & $8.1509 \mathrm{e}-05$ & $2.45534 \mathrm{e}+06$ & $5.1227 \mathrm{e}-05$ & Reject \\
\hline topic1965 & $6.2461 \mathrm{e}-05$ & $4.6536 \mathrm{e}-05$ & $2.02035 \mathrm{e}+06$ & $3.5084 \mathrm{e}-42$ & Reject \\
\hline topic1966 & $6.4129 \mathrm{e}-05$ & $5.2264 \mathrm{e}-05$ & $2.27404 \mathrm{e}+06$ & $1.1319 \mathrm{e}-15$ & Reject \\
\hline topic1967 & 0.00015806 & 0.00011075 & $1.85964 \mathrm{e}+06$ & $1.0199 \mathrm{e}-65$ & Reject \\
\hline topic1968 & 0.00010582 & $7.6344 \mathrm{e}-05$ & $2.14112 \mathrm{e}+06$ & 8.3921e-28 & Reject \\
\hline topic1969 & 0.00010293 & $6.9275 \mathrm{e}-05$ & $1.96985 \mathrm{e}+06$ & $7.211 \mathrm{e}-49$ & Reject \\
\hline topic1970 & 0.00018583 & 0.00016343 & $2.29363 \mathrm{e}+06$ & $3.4613 \mathrm{e}-14$ & Reject \\
\hline topic1971 & $9.283 \mathrm{e}-05$ & $8.716 \mathrm{e}-05$ & $2.44116 \mathrm{e}+06$ & $1.3299 \mathrm{e}-05$ & Reject \\
\hline topic1972 & 0.00011137 & $9.5248 \mathrm{e}-05$ & $2.31476 \mathrm{e}+06$ & $1.3358 \mathrm{e}-12$ & Reject \\
\hline topic1973 & $6.3132 \mathrm{e}-05$ & $4.6866 \mathrm{e}-05$ & $2.08642 \mathrm{e}+06$ & $4.0803 \mathrm{e}-33$ & Reject \\
\hline topic1974 & 0.00015752 & 0.00017243 & $2.48703 \mathrm{e}+06$ & 0.00094947 & Reject \\
\hline topic1975 & $2.8016 \mathrm{e}-05$ & 0.00010521 & $1.59874 \mathrm{e}+06$ & $4.9379 \mathrm{e}-75$ & Reject \\
\hline topic1976 & $8.5029 \mathrm{e}-05$ & $5.0896 \mathrm{e}-05$ & $1.70426 \mathrm{e}+06$ & $1.7115 \mathrm{e}-94$ & Reject \\
\hline topic1977 & 0.00012079 & $8.5383 \mathrm{e}-05$ & $2.1498 \mathrm{e}+06$ & $1.4134 \mathrm{e}-26$ & Reject \\
\hline topic1978 & 0.00011448 & $9.0594 \mathrm{e}-05$ & $2.11888 \mathrm{e}+06$ & $4.0151 \mathrm{e}-30$ & Reject \\
\hline topic1979 & 0.00011168 & $6.5824 \mathrm{e}-05$ & $1.89261 \mathrm{e}+06$ & $2.66 \mathrm{e}-59$ & Reject \\
\hline topic1980 & $4.6726 \mathrm{e}-05$ & $3.169 \mathrm{e}-05$ & $2.00123 \mathrm{e}+06$ & $9.3379 \mathrm{e}-45$ & Reject \\
\hline topic1981 & $9.7957 \mathrm{e}-05$ & 7.9304e-05 & $2.03213 \mathrm{e}+06$ & $1.6377 \mathrm{e}-40$ & Reject \\
\hline topic1982 & $9.1961 \mathrm{e}-05$ & $7.7854 \mathrm{e}-05$ & $2.52103 \mathrm{e}+06$ & 0.0089284 & Reject \\
\hline topic1983 & $4.7049 \mathrm{e}-05$ & $5.878 \mathrm{e}-05$ & $2.25765 \mathrm{e}+06$ & $5.6032 \mathrm{e}-17$ & Reject \\
\hline topic1984 & 0.00020754 & 0.00015533 & $2.00152 \mathrm{e}+06$ & $7.7192 \mathrm{e}-45$ & Reject \\
\hline topic1985 & 0.00010205 & $7.1105 \mathrm{e}-05$ & $2.2256 \mathrm{e}+06$ & $2.0398 \mathrm{e}-19$ & Reject \\
\hline topic1986 & $1.0003 \mathrm{e}-05$ & $7.6556 \mathrm{e}-06$ & $2.06632 \mathrm{e}+06$ & $1.4061 \mathrm{e}-28$ & Reject \\
\hline topic1987 & 0.00013503 & $8.8629 \mathrm{e}-05$ & $1.93941 \mathrm{e}+06$ & $2.0004 \mathrm{e}-53$ & Reject \\
\hline topic1988 & $8.1449 \mathrm{e}-05$ & $6.8648 \mathrm{e}-05$ & $2.34182 \mathrm{e}+06$ & $1.1077 \mathrm{e}-10$ & Reject \\
\hline topic1989 & 0.0001514 & 0.00014748 & $2.55897 \mathrm{e}+06$ & 0.098484 & Accept \\
\hline topic1990 & 0.00013717 & 0.00011206 & $2.2513 \mathrm{e}+06$ & $1.6849 \mathrm{e}-17$ & Reject \\
\hline topic1991 & 7.6038e-08 & $2.1119 \mathrm{e}-07$ & 578026 & $1.6993 \mathrm{e}-29$ & Reject \\
\hline topic1992 & 0.00012389 & $6.5241 \mathrm{e}-05$ & $2.17571 \mathrm{e}+06$ & $3.6502 \mathrm{e}-23$ & Reject \\
\hline topic1993 & 0.00010935 & $7.0551 \mathrm{e}-05$ & $1.66499 \mathrm{e}+06$ & $1.125 \mathrm{e}-102$ & Reject \\
\hline topic1994 & 0.00013247 & 0.00012317 & $2.52541 \mathrm{e}+06$ & 0.011449 & Reject \\
\hline topic1995 & 0.00013808 & 0.00012293 & $2.46814 \mathrm{e}+06$ & 0.00023114 & Reject \\
\hline topic1996 & 0.00012758 & 0.00011738 & $2.4522 \mathrm{e}+06$ & $4.732 \mathrm{e}-05$ & Reject \\
\hline topic1997 & $5.1255 \mathrm{e}-05$ & $4.6133 \mathrm{e}-05$ & $2.49639 \mathrm{e}+06$ & 0.0059384 & Reject \\
\hline topic1998 & $4.4144 \mathrm{e}-05$ & $2.7293 \mathrm{e}-05$ & $2.11704 \mathrm{e}+06$ & 5.7813e-30 & Reject \\
\hline topic1999 & $7.5208 \mathrm{e}-05$ & $6.0867 \mathrm{e}-05$ & $2.32746 \mathrm{e}+06$ & $1.5961 \mathrm{e}-11$ & Reject \\
\hline
\end{tabular}


Table A1 - Continued from previous page

\begin{tabular}{lrrrrl}
\hline Feature & EN median & FL median & u-value & p-value & $\mathbf{H}_{\mathbf{0}}$ \\
\hline gender & 1 & 1 & $2.35098 \mathrm{e}+06$ & $1.6547 \mathrm{e}-10$ & Reject \\
age & 23 & 25 & $1.95898 \mathrm{e}+06$ & $2.9456 \mathrm{e}-15$ & Reject \\
neg. sent. & 2 & 1 & 624502 & 0.0066989 & Reject \\
hapax & 739 & 586 & $1.85977 \mathrm{e}+06$ & $2.6195 \mathrm{e}-66$ & Reject \\
dis & 160 & 116 & $1.70626 \mathrm{e}+06$ & $1.9604 \mathrm{e}-94$ & Reject \\
M1 & 3963 & 3091 & $2.1844 \mathrm{e}+06$ & $1.6349 \mathrm{e}-23$ & Reject \\
M2 & 166660 & 123880 & $2.47331 \mathrm{e}+06$ & 0.00024699 & Reject \\
yule & 0.10891 & 0.087471 & $1.52566 \mathrm{e}+06$ & $5.1578 \mathrm{e}-134$ & Reject \\
sichel & 0.13771 & 0.12848 & $1.80145 \mathrm{e}+06$ & $2.1448 \mathrm{e}-76$ & Reject \\
brunet & 4017.1 & 3126.2 & $2.18192 \mathrm{e}+06$ & $9.3735 \mathrm{e}-24$ & Reject \\
honore & 1026.5 & 1006.5 & $2.3479 \mathrm{e}+06$ & $1.6979 \mathrm{e}-10$ & Reject \\
\hline
\end{tabular}




\section{CURRICULUM VITAE}

NAME: $\quad$ Kristopher Reese

ADDRESS: $\quad$ Department of Computer Science and Engineering University of Louisville

Louisville, KY 40292

EDUCATION: M.S., Computer Science

University of Louisville

2011

B.S., Computer Science

Hood College

2009

B.A., Music Performance

Hood College

2009

\section{TEACHING:}

\section{University of Louisville}

- Program Design in C (2009/2010)

- CIS Development Project (2013)

- Introduction to Computer Science (2013)

\section{Elizabethtown Community and Technical College}

- Introduction to Computers: Instructor (2010-2012)

- Computer Maintenance Essentials: Instructor (2010-2012)

- Advanced Computer Maintenance: Instructor (2010)

- Program Design and Development: Instructor (2011-2012) 
- Introduction to Database Design: Instructor (2011)

- Introduction to JavaScript: Instructor (2011)

\section{PROFESSIONAL EXPERIENCE:}

U.S. Government Dec. 2013 - Present

Technology Researcher \& Analyst

- Research in Biometrics, Identity Sciences, Materials Science, Computer Vision, and Computational Social Sciences.

University of Louisville Aug. 2012 - Dec. 2013

Enterprise Systems Developer III

- Acting as Lead Application Architect and Developer.

- Creating highly interactive Data Visualizations with thousands of data points using D3.js and other JavaScript frameworks for Healthcare analytics.

- Coding a highly secure and massive scale application for Healthcare data using Zend Framework 2.0, PHP, HTML5 and other Web Based Interface Development tools.

- Creation of an OpenID Provider and Identity Management System for user selfservice with OpenID endpoints for integration into main application system.

- Extensive use of Oracle SQL, PL/SQL, MySQL, and creation of RESTful APIs for enterprise application architectures.

- Testing of applications with PHPUnit for unit tests.

Alcorn State University Feb. 2012 - Aug. 2012

Visiting Researcher

- Support for biometrics research funded under the DoD and DHS.

- Research and develop new methods to enhance work in thermal facial recognition and night vision recolorization.

- Test and integrate all algorithms and components in the MATLAB prototype system.

- Write technical reports and publish research achievements.

Yakabod, Inc. Feb. 2008 - Aug. 2009

Web Programmer

- Worked on a Highly Secure application (The Yakabox), which is used by over 20,000 users worldwide and by government agencies such as the NSA.

- Researched and Developed Server- and Client- side systems for Large-Scale Information Retrieval and Enterprise Social Networking using various web-based languages, frameworks, Web Services, and APIs.

- Automated the updating process for the Yakabox. 


\section{PUBLICATIONS}

\section{Conference Papers \& Posters:}

Reese, K., R. Bessette, P. Hancock. 2013. "KnowYourColors: Visual Dashboards for Blood Metrics and Healthcare Analytics." In Proceedings of the IEEE International Symposium on Signal Processing and Information Technology (ISSPIT). pp. - . Athens, Greece. October 2013

Ouch, R., K. Reese, R. Yampolskiy. 2013. "Hybrid genetic algorithm for the maximum clique problem combining sharing and migration." In Proceedings of the 24th Midwest Artificial Intelligence and Cognitive Sciences Conference (MAICS '13). pp. - . New Albany, Indiana. April 2013

Zheng, Y., K. Reese, E. Blasch, P. McManamon. 2013. "Qualitative evaluations and comparisons of six night-vision colorization methods." In Proceedings of the International Society for Optics and Phototonics (SPIE). pp. - . Baltimore, Maryland. April 2013

Zheng, Y., A. Elmaghraby, K. Reese. 2012. "Performance Improvement of Face Recognition using Multispectral Images and Stereo Images." In Proceedings of the IEEE Symposium on Signal Processing and Information Technology (ISSPIT). pp. 280-285. Ho Chi Minh City, Vietnam. December 2012

Reese, K., R. Yampolskiy, A. Elmaghraby. 2012. "A Framework for Interactive Generation of Music for Games." In Proceedings of the 17th International Conference on Computer Games: AI, Animation, Mobile, Interactive Multimedia, Educational \& Serious Games (CGAMES '12). pp. 131-137. Louisville, Kentucky. August 2012

Reese, K., Y. Zheng, A. Elmaghraby. 2012. "A Comparison of Face Detection Algorithms in the Visible and Thermal Spectrums." In Proceedings of the International Conference on Advances in Computer Science and Application (CSA '12). pp. 49-53. Amsterdam, Netherlands. June 2012

Reese, K., A. Salem, G. Dimitoglou. 2009. "Gaming Concepts in Accessible HCI for Bare-Hand Computer Interaction." In Proceedings of the 14th International Conference on Computer Games: AI, Animation, Mobile, Interactive Multimedia, Educational \& Serious Games (CGAMES '09). pp. 40-46. Louisville, KY. August 2009

Reese, K., A. Salem, G. Dimitoglou. 2008. "Using Standard Deviation in Signal Strength Detection to Determine Jamming in Wireless Networks." In Proceedings of the 21st International Conference on Computer Applications in Industry and Engineering (CAINE '08). pp. 250-254. Honolulu, HI. December 2008 
Reese, K., G. Dimitoglou. 2008. "A Survey of Path Planning Algorithms for Autonomous Robotics." In Proceedings of the Consortium for Computer Sciences in Colleges, Eastern Conference 2008 (CCSCE '08). Frederick, MD. October 2008

\section{Journal Papers:}

Reese, K., M. Shields, (name omitted), (name omitted), A. Elmaghraby "Computational Behavioral Analytics: Emotion Analysis in Foreign Languages and Effects of Translations," Journal of IC Research \& Development (submitted), 2020 .

Reese, K., M. Shields, (name omitted), D. Woodard, A. Elmaghraby, "Computational Behavioral Analytics: The Effects of Translations on Estimation of Personality Traits," Journal of IC Research \& Development (submitted), 2020.

Reese, K., A. Salem. 2009. "A Survey on Jamming Avoidance in Wireless AdHoc Sensory Networks." Journal of Computing Sciences in Colleges (CCSCE '08). Vol. 24. Iss. 3. pp. 93-98. Frederick, MD. January 2009

\section{Theses:}

Reese, K., 2011. "Computationally Generated Music using Reinforcement Learning." University of Louisville (UL). Louisville, KY. May 2011

\section{PRESENTATIONS:}

Florida Institute for CyberSecurity Conference, February 28, 2018. "The Future of Identity Science." University of Florida, Gainesville, FL.

Joint DoD and DHS workshop on Image Analysis II, June 8, 2012. "A Technical Survey of Facial Recognition Techniques - Past Achievements and Future Applications." Alcorn State University, Alcorn, MS.

Doctoral Seminar, March 27, 2011. "Generative Chord Progressions using Reinforcement Learning." University of Louisville, Louisville, KY.

\section{CONFERENCE REVIEWER:}

- 2020 IEEE/IAPR International Joint Conference on Biometrics (IJCB)

- 2019 IEEE Biometrics: Theory, Applications, and Systems (BTAS)

\section{AWARDS:}

- Computer Science Undergraduate Achievement Award (2009), Hood College

- Oracle Open World Fusion Middleware Innovation Award (2012), Oracle 
- Exceptional Performance Awards from employers for research related efforts $(2016,2016,2016,2017,2017,2018,2019)$

GRANTS: Summer Research Institute

Co-Primary Investigator

Hood College

Frederick, MD, 2008

\section{PROFESSIONAL ASSOCIATIONS:}

Association for Computing Machinery (2007 - 2017)

Institute for Electrical and Electronics Engineers (2008 - Present)

American Mathematical Society (2010 - 2015)

Golden Key International Honor Society

International Neural Network Society (2015) 